\title{
Palladium-Catalyzed Regioselective Difluoroalkylation and Carbonylation of Alkynes
}

Qiang Wang, Yu-Tao He, Jia-Hui Zhao, Yi-Feng Qiu, Lan Zheng, Jing-Yuan Hu, Yu-Chen Yang, Xue-Yuan Liu, and Yong-Min Liang*

State Key Laboratory of Applied Organic Chemistry, Lanzhou University, Lanzhou 730000, P.R. China State Key Laboratory of Solid Lubrication, Lanzhou Institute of Chemical Physics, Chinese Academy of Sciences, Lanzhou, 730000, P.R. China

*E-mail: liangym@1zu.edu.cn.

\section{Table of Contents}

1 General Remarks $\quad$ S2

2 General Experimental Procedure S2

3 Optimization of The Reaction Conditions S2-S5

4 Characterization Data of 3aa-3va, 4ab-4ag and 4aj-4au, 5, 6 and $7 \quad$ S6-S22

$5 \quad$ Crystallographic data of 4au. $\quad$ S23-S24

$6{ }^{1} \mathrm{H}$ NMR、 ${ }^{13} \mathrm{C}$ NMR 、 ${ }^{19} \mathrm{~F}$ NMR Spectra for Substrates 3aa-3va, 4ab-4ag, S25-S87 4aj-4au, 5, 6 and 7 


\section{General Remarks}

All commercially available organic compounds were purchased from adamas-beta, Alfa Asar, and accelachem in China. Unless otherwise noted, reactions were carried out under an carbon monoxide atmosphere. DMF was distilled from $\mathrm{Mg}_{2} \mathrm{SO}_{4}$ under reduced pressure before used.

For Column chromatography, 200-300 mesh silica gel was employed. Analytical TLC was performed with silica gel GF254 plates. ${ }^{1} \mathrm{H}$ NMR $(400 \mathrm{MHz}),{ }^{13} \mathrm{C}$ NMR $(100 \mathrm{MHz})$ and ${ }^{19} \mathrm{~F}$ NMR $\left(376 \mathrm{MHz}\right.$ ) were recorded in $\mathrm{CDCl}_{3}$ using TMS as internal standard. IR spectra were recorded on a FT-IR spectrometer and only major peaks are reported in $\mathrm{cm}^{-1}$. All new products were further characterized by high resolution mass spectra (HRMS); copies of their ${ }^{1} \mathrm{H}$ NMR, ${ }^{13} \mathrm{C}$ NMR and ${ }^{19} \mathrm{~F}$ NMR spectra are provided. All solvents were dried under standard method.

\section{General experimental procedure}

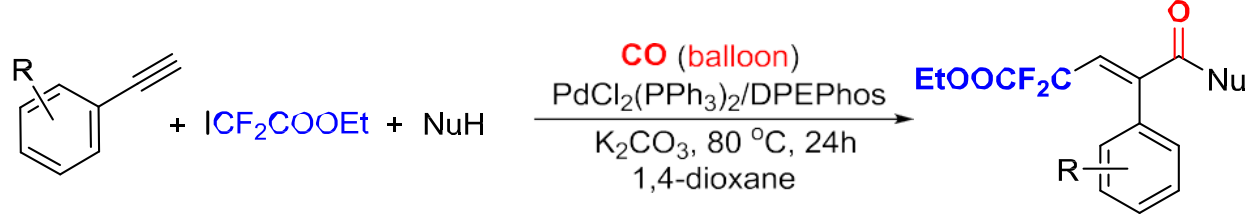

An oven-dried tube was charged with $\mathrm{K}_{2} \mathrm{CO}_{3}(0.30 \mathrm{mmol}, 1.5$ equiv), DPEPhos (10\% mmol $)$ and $\mathrm{PdCl}_{2}\left(\mathrm{PPh}_{3}\right)_{2}(5 \% \mathrm{mmol})$. The tube was evacuated and backfilled with $\mathrm{CO}$ (repeated three times). Then, 1,4-dioxane $(1.0 \mathrm{~mL})$ was injected after alkynes $(0.20 \mathrm{mmol}, 1.0$ equiv) ethyl difluoroiodoacetate $(0.34 \mathrm{mmol}, 1.7$ equiv $)$ and $\mathrm{NuH}(0.60 \mathrm{mmol}, 3.0$ equiv) were added into the tube. The reaction mixture was stirring at $80{ }^{\circ} \mathrm{C}$ for $24 \mathrm{~h}$. The balloon was then removed and the reaction mixture was cooled to room temperature. Water $(3 \mathrm{~mL})$ was added, and the product was extracted with DCM $(3 \times 10 \mathrm{~mL})$. The combined organic layers were washed with saturated brine, dried over $\mathrm{Na}_{2} \mathrm{SO}_{4}$, concentrated in vacuum and purified by flash column chromatography (silica gel) to afford the product.

\section{Optimization of Reaction Conditions}

\section{A: Optimization with Phenylacetylene As Substrate}

\section{A.1. Solvent Effect}

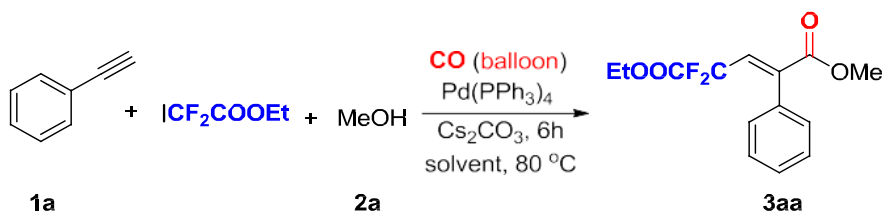

\begin{tabular}{|c|c|c|}
\hline \multicolumn{3}{|c|}{ Solvent Effect } \\
\hline Entry $^{a}$ & Solvent & Yield (\%) $^{b}$ \\
\hline 1 & toluene & 20 \\
\hline 2 & DMF & 0 \\
\hline 3 & DCE & 0 \\
\hline 4 & THF & 19 \\
\hline 5 & NMP & 19 \\
\hline 6 & $1,4-$ dioxane & 23 \\
\hline 7 & $\mathrm{Et}_{3} \mathrm{~N}$ & 0 \\
\hline 8 & $\mathrm{MeOH}$ & 0 \\
\hline
\end{tabular}




\begin{tabular}{l|c|c|}
\hline 9 & MeCN & 0 \\
\hline${ }^{a}$ Reaction conditions: $1 \mathrm{a}\left(0.10 \mathrm{mmol}, 1.0\right.$ equive), $\mathrm{ICF}_{2} \mathrm{COOEt}(0.17 \mathrm{mmol}, 1.7$ equiv), $\mathrm{MeOH}$ \\
$\left(1.5 \mathrm{mmol}, 1.5\right.$ equiv), $\mathrm{Cs}_{2} \mathrm{CO}_{3}\left(0.1 \mathrm{mmol}, 1.0\right.$ equiv), $\mathrm{Pd}\left(\mathrm{PPh}_{3}\right)_{4}(5 \mathrm{~mol} \%)$, solvent $(1.0 \mathrm{~mL})$, \\
$80^{\circ} \mathrm{C}, 6 \mathrm{~h}$, under CO balloon pressure). \\
${ }^{b}$ Betermined by GC (phenylate was used as internal standard). \\
\hline
\end{tabular}

\section{A.2. MeOH Effect}

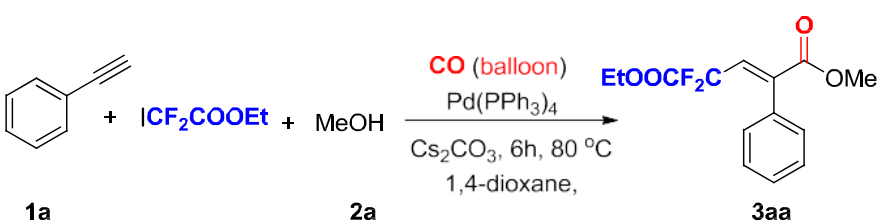

\begin{tabular}{|c|c|c|}
\hline \multicolumn{3}{|c|}{ MeOH Effect } \\
\hline Entry $^{a}$ & MeOH & Yield $(\%)^{b}$ \\
\hline 1 & 1.5 equiv & 23 \\
\hline 2 & 2.0 equiv & 29 \\
\hline 3 & 3.0 equiv & 32 \\
\hline $\begin{array}{l}{ }^{a} \text { Reaction conditions } \\
(0.10 \mathrm{mmol}, 1.0 \text { equi } \\
\text { pressure). }\end{array}$ & $\begin{array}{l}0 \text { equive), } I^{0} \mathrm{CF}_{2} \\
\text { ol \%), solvent (' }\end{array}$ & $\begin{array}{l}\text { ll, } 1.7 \text { equiv), } \\
\text { h, under } \mathrm{CO} \text { (b }\end{array}$ \\
\hline
\end{tabular}

\section{A.3. Temperature Effect}

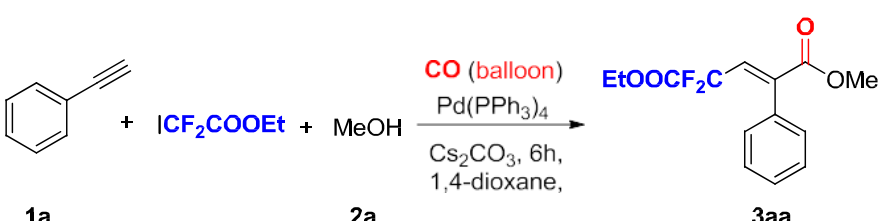

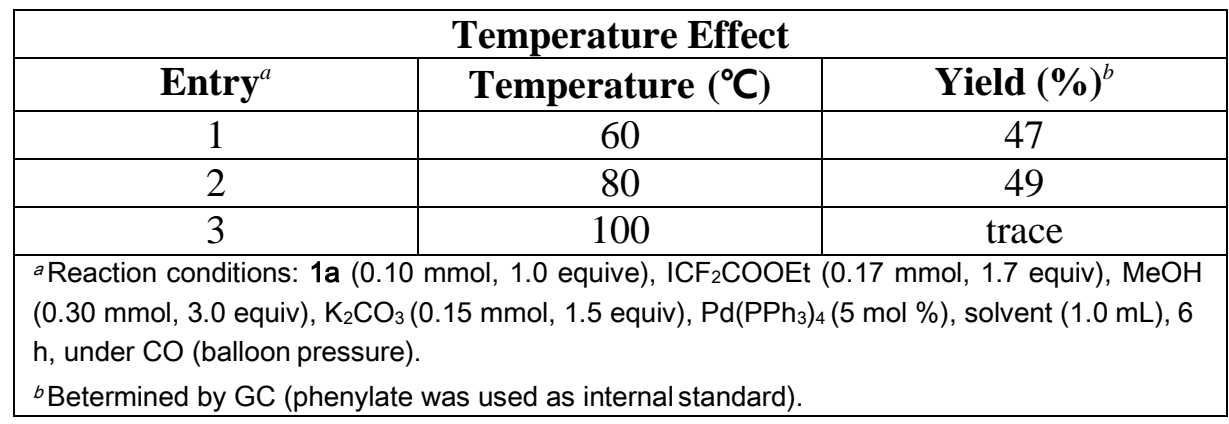

\section{A.4. Base Effect}

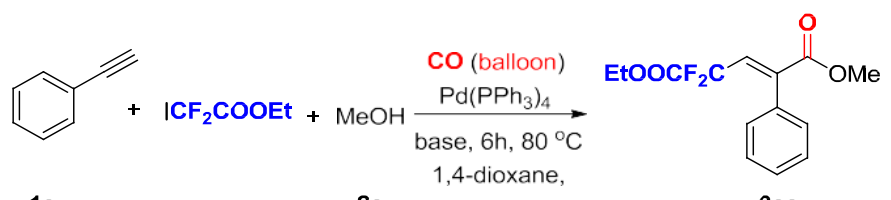

$1 a$

$2 a$

$3 a a$

\begin{tabular}{|c|c|c|}
\hline \multicolumn{3}{|c|}{ Base Effect } \\
\hline Entry $^{a}$ & Base (equiv) & Yield (\%) $^{b}$ \\
\hline 1 & $\mathrm{Na}_{2} \mathrm{CO}_{3}(1.0)$ & trace \\
\hline 2 & $\mathrm{~K}_{2} \mathrm{CO}_{3}(1.0)$ & 37 \\
\hline 3 & $\mathrm{Cs}_{2} \mathrm{CO}_{3}(1.0)$ & 32 \\
\hline
\end{tabular}




\section{A.7. Catalyst/Liagand Effect}

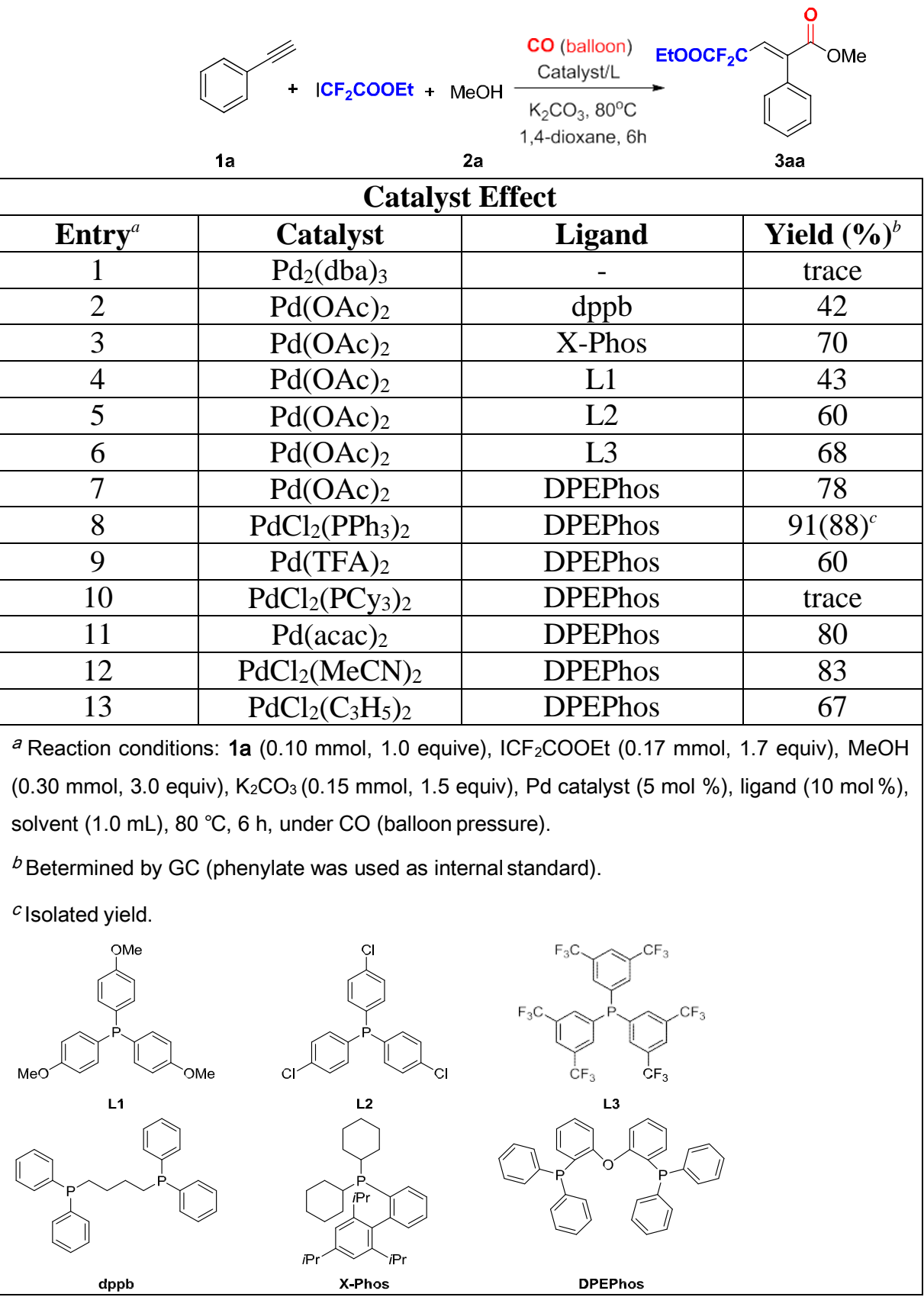


4. Characterization Data of 3aa-3va, 4ab-4ag and 4aj-4au, 5, 6 and 7.<smiles>CCOC(=O)CC=C(C(=O)OC)c1ccccc1</smiles>

(E)-5-ethyl 1-methyl 4,4-difluoro-2-phenylpent-2-enedioate

Yellow oil, $50.0 \mathrm{mg}, 88 \%$ yield, E/Z>20:1.

${ }^{1}$ H NMR (400 MHz, CDCl $) \delta$ ppm $7.37-7.36(\mathrm{~m}, 3 \mathrm{H}), 7.21-7.19(\mathrm{~m}, 2 \mathrm{H}), 7.05(\mathrm{t}, J=$ $11.4 \mathrm{~Hz}, 1 \mathrm{H}), 3.92(\mathrm{q}, J=7.2 \mathrm{~Hz}, 2 \mathrm{H}), 3.80(\mathrm{~s}, 4 \mathrm{H}), 1.17(\mathrm{t}, J=7.2 \mathrm{~Hz}, 3 \mathrm{H})$.

${ }^{13}$ C NMR (100 MHz, CDCl $) \delta$ ppm 166.0, $162.3(\mathrm{t}, J=32.8 \mathrm{~Hz}), 141.2(\mathrm{t}, J=8.6 \mathrm{~Hz})$, 132.1, 131.7 (t, $J=28.7 \mathrm{~Hz}), 129.4,128.9,127.8,111.5(\mathrm{t}, J=245.7 \mathrm{~Hz}), 63.1,53.0,13.6$.

${ }^{19}$ F NMR (376 MHz, CDCl 3$) \delta$ ppm $-99.9(\mathrm{~d}, J=7.5 \mathrm{~Hz})$.

IR (neat, $\mathrm{cm}^{-1}$ ): 3061, 2957, 1776, 1729, 1654, 1497, 1445, 1254, 1200, 1108, 1087, 1015, $774,700$.

HRMS (ESI) Calcd. for $\mathrm{C}_{14} \mathrm{H}_{14} \mathrm{~F}_{2} \mathrm{O}_{4}:[\mathrm{M}]+\mathrm{Na}=307.0752$. Found: 307.0755 .<smiles>CCOC(F)(F)F</smiles>

(E)-5-ethyl 1-methyl 4,4-difluoro-2-(p-tolyl)pent-2-enedioate

Brown oil, $47.1 \mathrm{mg}, 79 \%$ yield, $\mathrm{E} / \mathrm{Z}=17: 1$.

${ }^{1} \mathbf{H}$ NMR (400 MHz, CDCl$) \delta$ ppm 7.17 (d, $\left.J=8.0 \mathrm{~Hz}, 2 \mathrm{H}\right), 7.09(\mathrm{~d}, J=7.6 \mathrm{~Hz}, 2 \mathrm{H}), 7.02$ $(\mathrm{t}, J=11.2 \mathrm{~Hz}, 1 \mathrm{H}), 3.93(\mathrm{q}, J=7.2 \mathrm{~Hz}, 2 \mathrm{H}), 3.79(\mathrm{~s}, 3 \mathrm{H}), 2.36(\mathrm{~s}, 3 \mathrm{H}), 1.17(\mathrm{t}, J=7.2 \mathrm{~Hz}$, $3 \mathrm{H})$.

${ }^{13}$ C NMR (100 MHz, CDCl $) \delta$ ppm 166.2, $162.3(\mathrm{t}, J=32.8 \mathrm{~Hz}), 141.3(\mathrm{t}, J=8.7 \mathrm{~Hz})$, $138.9,131.4(\mathrm{t}, J=28.8 \mathrm{~Hz}), 129.3,129.2,128.5,111.6(\mathrm{t}, J=245.5 \mathrm{~Hz}), 63.1,52.9,21.3$, 13.6.

${ }^{19}$ F NMR (376 MHz, CDCl 3$) \delta$ ppm $-94.5(\mathrm{~d}, J=3.8 \mathrm{~Hz})$.

IR (neat, $\left.\mathrm{cm}^{-1}\right): 3031,2956,1776,1729,1651,1514,1437,1254,1200,1108,1083,1014$, 826.

HRMS (ESI) Calcd. for $\mathrm{C}_{15} \mathrm{H}_{16} \mathrm{~F}_{2} \mathrm{O}_{4}:[\mathrm{M}]+\mathrm{Na}=321.0909$. Found: 321.0914.<smiles>CCOC(=O)CC=C(C(=O)OC)c1ccc(Br)cc1</smiles> 
(E)-5-ethyl 1-methyl 2-(4-(tert-butyl)phenyl)-4,4-difluoropent-2-enedioate Yellow oil, $53.0 \mathrm{mg}, 78 \%$ yield, $\mathrm{E} / \mathrm{Z}=12: 1$.

${ }^{1} \mathbf{H}$ NMR (400 MHz, CDCl $) \delta$ ppm 7.37 (d, $\left.J=8.4 \mathrm{~Hz}, 2 \mathrm{H}\right), 7.14$ (d, $\left.J=8.4 \mathrm{~Hz}, 2 \mathrm{H}\right), 7.01$ $(\mathrm{t}, J=11.2 \mathrm{~Hz}, 1 \mathrm{H}), 3.84(\mathrm{q}, J=7.2 \mathrm{~Hz}, 2 \mathrm{H}), 3.80(\mathrm{~s}, 3 \mathrm{H}), 1.32(\mathrm{~s}, 9 \mathrm{H}), 1.13(\mathrm{t}, J=7.2 \mathrm{~Hz}$, $3 \mathrm{H})$.

${ }^{13}$ C NMR (100 MHz, CDCl $) \delta$ ppm 166.3, $162.3(\mathrm{t}, J=32.6 \mathrm{~Hz}), 152.0,141.2(\mathrm{t}, J=8.9$ $\mathrm{Hz}), 131.3$ (t, $J=29.1 \mathrm{~Hz}), 129.2,129.1,124.7,111.7$ (t, $J=245.0 \mathrm{~Hz}), 62.9,52.9,34.7$, 31.2, 13.6 .

${ }^{19}$ F NMR (376 MHz, CDCl 3 ) $\delta$ ppm -93.7.

IR (neat, $\mathrm{cm}^{-1}$ ):3036, 2963, 1776, 1730, 1651, 1512, 1437, 1254, 1202, 1112, 1081, 842.

HRMS (ESI) Calcd. for $\mathrm{C}_{18} \mathrm{H}_{22} \mathrm{~F}_{2} \mathrm{O}_{4}:[\mathrm{M}]+\mathrm{Na}=363.1378$. Found: 363.1384 .<smiles>CCCc1ccc(C(=CCC(=O)OCC)C(=O)OC)cc1</smiles>

(E)-5-ethyl 1-methyl 4,4-difluoro-2-(4-propylphenyl)pent-2-enedioate

Yellow oil, $49.6 \mathrm{mg}, 67 \%$ yield, $\mathrm{E} / \mathrm{Z}=13: 1$.

${ }^{1} \mathbf{H}$ NMR (400 MHz, CDCl $) \delta$ ppm $7.16(\mathrm{~d}, J=8.2 \mathrm{~Hz}, 2 \mathrm{H}), 7.11(\mathrm{~d}, J=8.2 \mathrm{~Hz}, 2 \mathrm{H}), 7.01$ $(\mathrm{t}, J=11.2 \mathrm{~Hz}, 1 \mathrm{H}), 3.88(\mathrm{q}, J=7.2 \mathrm{~Hz}, 2 \mathrm{H}), 3.80(\mathrm{~s}, 3 \mathrm{H}), 2.61-2.57(\mathrm{~m}, 2 \mathrm{H}), 1.64(\mathrm{~h}, J=$ $7.2 \mathrm{~Hz}, 2 \mathrm{H}), 1.15(\mathrm{t}, J=7.2 \mathrm{~Hz}, 3 \mathrm{H}), 0.95(\mathrm{t}, J=7.2 \mathrm{~Hz}, 3 \mathrm{H})$.

${ }^{13}$ C NMR (100 MHz, CDCl $) \delta$ ppm 166.3, $162.3(\mathrm{t}, J=32.6 \mathrm{~Hz}), 143.7,141.3(\mathrm{t}, J=8.9$ $\mathrm{Hz}), 131.4(\mathrm{t}, J=28.8 \mathrm{~Hz}), 129.4,127.9,127.9,111.6(\mathrm{t}, J=245.0 \mathrm{~Hz}), 63.0,52.9,37.8$, 24.3, 13.8, 13.6.

${ }^{19}$ F NMR (376 MHz, $\left.\mathbf{C D C l}_{3}\right) \delta$ ppm -94.1.

IR (neat, $\mathrm{cm}^{-1}$ ): 3030, 2959, 1776, 1729, 1651, 1610, 1512, 1437, 1254, 1200, 1108, 1082, $1015,820$.

HRMS (ESI) Calcd. for $\mathrm{C}_{17} \mathrm{H}_{20} \mathrm{~F}_{2} \mathrm{O}_{4}:[\mathrm{M}]+\mathrm{Na}=349.1222$. Found: 349.1226.<smiles>CCOC(=O)CC=C(C(=O)OC)c1ccc(OC)cc1</smiles>

(E)-5-ethyl 1-methyl 4,4-difluoro-2-(4-methoxyphenyl)pent-2-enedioate Yellow oil, $39.6 \mathrm{mg}, 63 \%$ yield, E/Z>20:1.

${ }^{1} \mathbf{H}$ NMR (400 MHz, CDCl 3$) \delta$ ppm $7.14(\mathrm{~d}, J=8.8 \mathrm{~Hz}, 2 \mathrm{H}), 7.00(\mathrm{t}, J=11.2 \mathrm{~Hz}, 1 \mathrm{H}), 6.88$ $(\mathrm{d}, J=8.4 \mathrm{~Hz}, 2 \mathrm{H}), 3.93$ (q, $J=7.2 \mathrm{~Hz}, 2 \mathrm{H}), 3.81(\mathrm{~s}, 3 \mathrm{H}), 3.80(\mathrm{~s}, 3 \mathrm{H}), 1.17$ (t, $J=7.2 \mathrm{~Hz}$, $3 \mathrm{H})$.

${ }^{13}$ C NMR (100 MHz, CDCl $) \delta$ ppm 166.3, $162.2(\mathrm{t}, J=32.9 \mathrm{~Hz}), 160.1,140.9(\mathrm{t}, J=8.7$ $\mathrm{Hz}), 131.2(\mathrm{t}, J=28.6 \mathrm{~Hz}), 130.9,124.2,113.3,111.7$ (t, $J=245.0 \mathrm{~Hz}), 63.1,55.2,52.9$, 13.6.

${ }^{19}$ F NMR (376 MHz, CDCl 3$) \delta$ ppm $-93.9(\mathrm{~d}, J=3.8 \mathrm{~Hz})$. 
IR (neat, $\mathrm{cm}^{-1}$ ): 2957, 1774, 1727, 1649, 1610, 1514, 1465, 1439, 1251, 1200, 1108, 1082, $1032,837$.

HRMS (ESI) Calcd. for $\mathrm{C}_{15} \mathrm{H}_{16} \mathrm{~F}_{2} \mathrm{O}_{5}:[\mathrm{M}]+\mathrm{Na}=337.0858$. Found: 337.0862 .<smiles>CCCCCOc1ccc(/C(=C\C(=O)OCC)C(=O)OC)cc1</smiles>

(E)-5-ethyl 1-methyl 4,4-difluoro-2-(4-(pentyloxy)phenyl)pent-2-enedioate Yellow oil, $59.9 \mathrm{mg}, 81 \%$ yield, E/Z>20:1.

${ }^{1}$ H NMR $(400$ MHz, CDCl $) \delta$ ppm $7.13(\mathrm{~d}, J=8.8 \mathrm{~Hz}, 2 \mathrm{H}), 7.00(\mathrm{t}, J=11.2 \mathrm{~Hz}, 1 \mathrm{H}), 6.86$ $(\mathrm{d}, J=8.8 \mathrm{~Hz}, 2 \mathrm{H}), 3.97-3.89(\mathrm{~m}, 4 \mathrm{H}), 3.80(\mathrm{~s}, 3 \mathrm{H}), 1.79(\mathrm{p}, J=6.8 \mathrm{~Hz}, 2 \mathrm{H}), 1.44-1.36$ $(\mathrm{m}, 4 \mathrm{H}), 1.16(\mathrm{t}, J=7.2 \mathrm{~Hz}, 3 \mathrm{H}), 0.93(\mathrm{t}, J=6.8 \mathrm{~Hz}, 3 \mathrm{H})$.

${ }^{13}$ C NMR (100 MHz, CDCl $) \delta$ ppm 166.3, $162.3(\mathrm{t}, J=32.8 \mathrm{~Hz}), 159.7,141.0(\mathrm{t}, J=8.9$ $\mathrm{Hz}), 131.1(\mathrm{t}, J=28.9 \mathrm{~Hz}), 130.9,123.9,113.7,111.7(\mathrm{t}, J=244.8 \mathrm{~Hz}), 67.9,63.0,52.9$, 28.8, 28.1, 22.4, 14.0, 13.6.

${ }^{19}$ F NMR (376 MHz, $\left.\mathbf{C D C l}_{3}\right) \delta$ ppm -93.8.

IR (neat, $\mathrm{cm}^{-1}$ ): 3043, 2957, 1776, 1729, 1649, 1609, 1512, 1470, 1437, 1252, 1198, 1108 , 1080, 1022, 836.

HRMS (ESI) Calcd. for $\mathrm{C}_{19} \mathrm{H}_{24} \mathrm{~F}_{2} \mathrm{O}_{5}:[\mathrm{M}]+\mathrm{Na}=393.1484$. Found: 393.1490 .<smiles>CCOC(=O)CC=C(C(=O)OC)c1cc(C)c(C)cc1C</smiles>

(E)-5-ethyl 1-methyl 4,4-difluoro-2-(2,4,5-trimethylphenyl)pent-2-enedioate Yellow oil, $54.8 \mathrm{mg}, 84 \%$ yield, E/Z>20:1.

${ }^{1}$ H NMR (400 MHz, CDCl $) \delta$ ppm $7.06(\mathrm{t}, J=11.2 \mathrm{~Hz}, 1 \mathrm{H}), 6.95$ (s, 1H), $6.78(\mathrm{~s}, 1 \mathrm{H})$, $3.90(\mathrm{q}, J=7.2 \mathrm{~Hz}, 2 \mathrm{H}), 3.77(\mathrm{~s}, 3 \mathrm{H}), 2.22(\mathrm{~s}, 3 \mathrm{H}), 2.20(\mathrm{~s}, 3 \mathrm{H}), 2.06(\mathrm{~s}, 3 \mathrm{H}), 1.18(\mathrm{t}, J=7.2$ $\mathrm{Hz}, 3 \mathrm{H})$.

${ }^{13}$ C NMR (100 MHz, CDCl $) \delta$ ppm 166.1, $162.3(\mathrm{t}, J=32.6 \mathrm{~Hz}), 141.2(\mathrm{t}, J=8.8 \mathrm{~Hz})$, $137.4,133.7,133.3,132.0(\mathrm{t}, J=28.7 \mathrm{~Hz}), 131.1,130.7,129.1,111.5(\mathrm{t}, J=245.8 \mathrm{~Hz})$, 63.0, 52.9, 19.5, 19.1, 19.0, 13.5.

${ }^{19}$ F NMR (376 MHz, CDCl 3 ) $\delta$ ppm $-94.0--99.2(\mathrm{~m})$.

IR (neat, $\mathrm{cm}^{-1}$ ): 2954, 1776, 1760, 1729, 1655, 1505, 1450, 1437, 1255, 1196, 1123, 1092, 1010, 856, 793, 771.

HRMS (ESI) Calcd. for $\mathrm{C}_{17} \mathrm{H}_{20} \mathrm{~F}_{2} \mathrm{O}_{4}:[\mathrm{M}]+\mathrm{Na}=349.1222$. Found: 349.1225 .<smiles>CCOC(=O)CC=C(C(=O)OC)c1ccc(F)cc1</smiles> 
(E)-5-ethyl 1-methyl 4,4-difluoro-2-(4-fluorophenyl)pent-2-enedioate

Brown oil, $52.5 \mathrm{mg}, 87 \%$ yield, $\mathrm{E} / \mathrm{Z}=12: 1$.

${ }^{1} \mathbf{H}$ NMR (400 MHz, CDCl $) \delta$ ppm $7.21-7.18$ (m, 2H), $7.08-7.03$ (m, 3H), 4.01 (q, $J=$ $7.2 \mathrm{~Hz}, 2 \mathrm{H}), 3.81(\mathrm{~s}, 3 \mathrm{H}), 1.21(\mathrm{t}, J=7.2 \mathrm{~Hz}, 3 \mathrm{H})$.

${ }^{13}$ C NMR (100 MHz, CDCl 3 ) $\delta$ ppm $165.1(\mathrm{~d}, J=163.5 \mathrm{~Hz}), 162.3(\mathrm{t}, J=32.8 \mathrm{~Hz}), 161.8$, $140.3(\mathrm{t}, J=8.1 \mathrm{~Hz}), 132.0(\mathrm{t}, J=28.2 \mathrm{~Hz}), 131.4(\mathrm{~d}, J=8.1 \mathrm{~Hz}), 128.1(\mathrm{~d}, J=3.6 \mathrm{~Hz})$, $115.0(\mathrm{~d}, J=21.7 \mathrm{~Hz}), 111.5(\mathrm{t}, J=246.3 \mathrm{~Hz}), 63.2,53.0,13.7$.

${ }^{19}$ F NMR (376 MHz, CDCl3) $\delta$ ppm $-95.2(\mathrm{~d}, \mathrm{~J}=7.5 \mathrm{~Hz}),-112.0$.

IR $\left(\right.$ neat, $\left.\mathrm{cm}^{-1}\right): 3074,2958,1775,1729,1655,1604,1512,1438,1256,1226,1108,1084$, $1018,843$.

HRMS (ESI) Calcd. for $\mathrm{C}_{14} \mathrm{H}_{13} \mathrm{~F}_{3} \mathrm{O}_{4}:[\mathrm{M}]+\mathrm{Na}=325.0658$. Found: 325.0661 .<smiles>CCOC(=O)CC=C(C(=O)OC)c1ccc(Cl)cc1</smiles>

(E)-5-ethyl 1-methyl 2-(4-chlorophenyl)-4,4-difluoropent-2-enedioate Yellow oil, $43.9 \mathrm{mg}, 69 \%$ yield, E/Z>20:1.

${ }^{1} \mathbf{H}$ NMR (400 MHz, CDCl 3 ) $\delta$ ppm $7.34(\mathrm{~d}, \mathrm{~J}=8.4 \mathrm{~Hz}, 2 \mathrm{H}), 7.15(\mathrm{~d}, \mathrm{~J}=8.4 \mathrm{~Hz}, 2 \mathrm{H}), 7.07$ $(\mathrm{t}, \mathrm{J}=11.6 \mathrm{~Hz}, 1 \mathrm{H}), 4.02(\mathrm{q}, \mathrm{J}=7.2 \mathrm{~Hz}, 2 \mathrm{H}), 3.80(\mathrm{~s}, 3 \mathrm{H}), 1.22(\mathrm{t}, \mathrm{J}=7.2 \mathrm{~Hz}, 3 \mathrm{H})$.

${ }^{13}$ C NMR (100 MHz, CDCl $) \delta$ ppm 165.7, $162.3(\mathrm{t}, J=32.7 \mathrm{~Hz}), 140.1(\mathrm{t}, J=7.9 \mathrm{~Hz})$, 135.1, 132.1 (t, $J=28.1 \mathrm{~Hz}), 130.8,130.6,128.1,111.4(\mathrm{t}, J=246.8 \mathrm{~Hz}), 63.3,53.1,13.6$.

${ }^{19}$ F NMR (376 MHz, CDCl 3 ) $\delta$ ppm $-95.48(\mathrm{~d}, J=3.8 \mathrm{~Hz})$.

IR $\left(\right.$ neat, $\left.\mathrm{cm}^{-1}\right)$ : 3067, 2957, 1775, 1729, 1655, 1596, 1493, 1438, 1254, 1198, 1103, 1091, $1017,855$.

HRMS (ESI) Calcd. for $\mathrm{C}_{14} \mathrm{H}_{13} \mathrm{ClF}_{2} \mathrm{O}_{4}:[\mathrm{M}]+\mathrm{Na}=341.0363$. Found: 341.0367.<smiles>CCOC(=O)C=C(C(=O)OC)c1ccc(Br)cc1</smiles>

(E)-5-ethyl 1-methyl 2-(4-bromophenyl)-4,4-difluoropent-2-enedioate Yellow oil, $42.0 \mathrm{mg}, 58 \%$ yield, E/Z>20:1.

${ }^{1}$ H NMR (400 MHz, CDCl$) \delta$ ppm $7.50(\mathrm{~d}, J=8.4 \mathrm{~Hz}, 2 \mathrm{H}), 7.13-7.00(\mathrm{~m}, 3 \mathrm{H}), 4.03 \quad$ (q, $J=7.2 \mathrm{~Hz}, 3 \mathrm{H}), 3.80(\mathrm{~s}, 3 \mathrm{H}), 1.22(\mathrm{t}, J=7.2 \mathrm{~Hz}, 3 \mathrm{H})$.

${ }^{13}$ C NMR (100 MHz, CDCl 3$) \delta$ ppm 165.6, $162.3(\mathrm{t}, J=32.7 \mathrm{~Hz}), 140.1(\mathrm{t}, J=7.9 \mathrm{~Hz})$, $132.1(\mathrm{t}, J=28.1 \mathrm{~Hz}), 131.1,131.1,131.0,123.4,111.4(\mathrm{t}, J=246.9 \mathrm{~Hz}), 63.4,53.1,13.7$.

${ }^{19}$ F NMR (376 MHz, CDCl 3$) \delta$ ppm $-95.6(\mathrm{~d}, J=7.5 \mathrm{~Hz})$.

IR (neat, $\left.\mathrm{cm}^{-1}\right): 3068,2956,1775,1729,1655,1591,1488,1437,1256,1203,1107,1083$, $1012,842$.

HRMS (ESI) Calcd. for $\mathrm{C}_{14} \mathrm{H}_{13} \mathrm{BrF}_{2} \mathrm{O}_{4}:[\mathrm{M}]+\mathrm{Na}=384.9857$. Found: 384.9864 . 
<smiles>CCOC(=O)CC=C(C(=O)OC)c1ccc(C(F)(F)F)cc1</smiles>

(E)-5-ethyl 1-methyl 4,4-difluoro-2-(4-(trifluoromethyl)phenyl)pent-2-enedioate Brown oil, $45.0 \mathrm{mg}, 64 \%$ yield, $\mathrm{E} / \mathrm{Z}=20: 1$.

${ }^{1} \mathrm{H}$ NMR $(400 \mathrm{MHz}, \mathrm{CDCl} 3) \delta \mathrm{ppm} 7.63(\mathrm{~d}, J=8.0 \mathrm{~Hz}, 2 \mathrm{H}), 7.34(\mathrm{~d}, J=8.0 \mathrm{~Hz}, 2 \mathrm{H}), 7.12$ $(\mathrm{t}, J=11.6 \mathrm{~Hz}, 1 \mathrm{H}), 4.04(\mathrm{q}, J=7.2 \mathrm{~Hz}, 2 \mathrm{H}), 3.81(\mathrm{~s}, 3 \mathrm{H}), 1.21(\mathrm{t}, J=7.2 \mathrm{~Hz}, 3 \mathrm{H})$.

${ }^{13} \mathrm{C}$ NMR (100 MHz, CDCl3) $\delta \mathrm{ppm} 165.4,162.3(\mathrm{t}, J=32.6 \mathrm{~Hz}), 140.0(\mathrm{t}, J=7.3 \mathrm{~Hz})$, 136.0, $132.5(\mathrm{t}, J=27.7 \mathrm{~Hz}), 130.9(\mathrm{q}, J=32.3 \mathrm{~Hz}), 129.8,124.8(\mathrm{q}, J=3.6 \mathrm{~Hz}), 123.9$ (d, $J=270.6 \mathrm{~Hz}), 111.3(\mathrm{t}, J=247.6 \mathrm{~Hz}), 63.4,53.2,13.6$.

${ }^{19} \mathrm{~F}$ NMR (376 MHz, CDCl3) $\delta$ ppm -62.87, -96.23.

IR (neat, $\mathrm{cm}^{-1}$ ): 2960, 1775, 1731, 1656, 1619, 1439, 1326, 1258, 1213, 1127, 1089, 1021, 850 .

HRMS (ESI) Calcd. for $\mathrm{C}_{15} \mathrm{H}_{13} \mathrm{~F}_{5} \mathrm{O}_{4}:[\mathrm{M}]+\mathrm{Na}=375.0626$. Found: 375.0632.<smiles>CCOC(=O)CC=C(C(=O)OC)c1ccc(-c2ccccc2)cc1</smiles>

(E)-5-ethyl 1-methyl 2-([1,1'-biphenyl]-4-yl)-4,4-difluoropent-2-enedioate Yellow oil, $48.2 \mathrm{mg}, 67 \%$ yield, $\mathrm{E} / \mathrm{Z}=12: 1$.

${ }^{1} \mathbf{H}$ NMR (400 MHz, CDCl 3$) \delta$ ppm $7.61-7.58(\mathrm{~m}, 4 \mathrm{H}), 7.45(\mathrm{t}, J=7.2 \mathrm{~Hz}, 2 \mathrm{H}), 7.36(\mathrm{t}, J$ $=7.2 \mathrm{~Hz}, 1 \mathrm{H}), 7.28(\mathrm{~d}, J=8.4 \mathrm{~Hz}, 2 \mathrm{H}), 7.07(\mathrm{t}, J=11.6 \mathrm{~Hz}, 1 \mathrm{H}), 3.95(\mathrm{q}, J=7.2 \mathrm{~Hz}, 2 \mathrm{H})$, $3.83(\mathrm{~s}, 3 \mathrm{H}), 1.16(\mathrm{t}, J=7.2 \mathrm{~Hz}, 3 \mathrm{H})$.

${ }^{13}$ C NMR (100 MHz, CDCl $) \delta$ ppm 166.1, $162.4(\mathrm{t}, J=32.8 \mathrm{~Hz}), 141.7,140.9(\mathrm{t}, J=8.6$ $\mathrm{Hz}), 140.2,131.7(\mathrm{t}, J=28.8 \mathrm{~Hz}), 131.1,129.9,128.8,127.7,127.1,126.5,111.6(\mathrm{t}, J=$ $245.9 \mathrm{~Hz}), 63.2,53.0,13.6$.

${ }^{19}$ F NMR (376 MHz, CDCl 3$) \delta$ ppm $-94.6(\mathrm{~d}, J=3.8 \mathrm{~Hz})$.

IR (neat, cm-1): 3059, 2955, 1775, 1729, 1653, 1488, 1448, 1437, 1254, 1202, 1108, 1083, $1011,847,699$.

HRMS (ESI) Calcd. for $\mathrm{C}_{20} \mathrm{H}_{18} \mathrm{~F}_{2} \mathrm{O}_{4}$ : $[\mathrm{M}]+\mathrm{Na}=383.1065$. Found: 383.1069 .<smiles>CCOC(=O)CC=C(C(=O)OC)c1cccc(C)c1</smiles>

(E)-5-ethyl 1-methyl 4,4-difluoro-2-(m-tolyl)pent-2-enedioate Yellow oil, $44.7 \mathrm{mg}, 75 \%$ yield, $\mathrm{E} / \mathrm{Z}=16: 1$.

${ }^{1} \mathbf{H}$ NMR (400 MHz, $\left.\mathbf{C D C l}_{3}\right) \delta$ ppm $7.27-7.23(\mathrm{~m}, 1 \mathrm{H}), 7.18(\mathrm{~d}, J=7.6 \mathrm{~Hz}, 1 \mathrm{H}), 7.06-$ $7.00(\mathrm{~m}, 3 \mathrm{H}), 3.90(\mathrm{q}, J=7.2 \mathrm{~Hz}, 2 \mathrm{H}), 3.80(\mathrm{~s}, 3 \mathrm{H}), 2.35$ (s, 3H), 1.17 (t, $J=7.2 \mathrm{~Hz}, 3 \mathrm{H})$. 
${ }^{13}$ C NMR (100 MHz, $\left.\mathbf{C D C l}_{3}\right) \delta$ ppm 166.1, $162.2(\mathrm{t}, J=32.8 \mathrm{~Hz}), 141.3(\mathrm{t}, J=8.7 \mathrm{~Hz})$, $137.5,132.0,131.5(\mathrm{t}, J=28.8 \mathrm{~Hz}), 129.9,129.7,127.7,126.5,111.5(\mathrm{t}, J=245.4 \mathrm{~Hz})$, 63.0, 53.0, 21.3, 13.6.

${ }^{19}$ F NMR (376 MHz, $\left.\mathbf{C D C l}_{3}\right) \delta$ ppm -94.5.

IR (neat, $\mathrm{cm}^{-1}$ ): 2956, 1775, 1728, 1655, 1605, 1437, 1253, 1229, 1109, 1083, 1026, 785, 706.

HRMS (ESI) Calcd. for $\mathrm{C}_{15} \mathrm{H}_{16} \mathrm{~F}_{2} \mathrm{O}_{4}:[\mathrm{M}]+\mathrm{Na}=321.0909$. Found: 321.0913.<smiles>CCOC(=O)C=C(C(=O)OC)c1cccc(OC)c1</smiles>

(E)-5-ethyl 1-methyl 4,4-difluoro-2-(3-methoxyphenyl)pent-2-enedioate Yellow oil, $40.2 \mathrm{mg}, 64 \%$ yield, $\mathrm{E} / \mathrm{Z}=11: 1$.

${ }^{1} \mathbf{H}$ NMR (400 MHz, CDCl 3$) \delta$ ppm $7.29-7.25(\mathrm{~m}, 1 \mathrm{H}), 7.03(\mathrm{t}, J=11.2 \mathrm{~Hz}, 1 \mathrm{H}), 6.92-$ $6.90(\mathrm{~m}, 1 \mathrm{H}), 6.78(\mathrm{~d}, J=7.2 \mathrm{~Hz}, 1 \mathrm{H}), 6.74-6.73(\mathrm{~m}, 1 \mathrm{H}), 3.95(\mathrm{q}, J=7.2 \mathrm{~Hz}, 2 \mathrm{H}), 3.80$ $(\mathrm{s}, 6 \mathrm{H}), 1.18(\mathrm{t}, J=7.2 \mathrm{~Hz}, 3 \mathrm{H})$.

${ }^{13}$ C NMR (100 MHz, CDCl $) \delta$ ppm 166.0, $162.3(\mathrm{t}, J=32.9 \mathrm{~Hz}), 158.9,141.0(\mathrm{t}, J=8.5$ $\mathrm{Hz}), 133.3,131.7(\mathrm{t}, J=28.9 \mathrm{~Hz}), 128.9,121.7,115.0,114.7,111.5(\mathrm{t}, J=245.6 \mathrm{~Hz}), 63.1$, 55.2, 53.0, 13.6 .

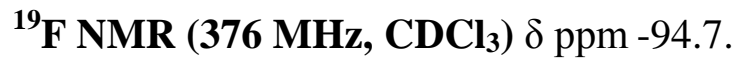

IR (neat, $\mathrm{cm}^{-1}$ ): 3066, 2957, 1775, 1728, 1654, 1601, 1580, 1490, 1435, 1254, 1201, 1108, 1081, 1021, 786, 704.

HRMS (ESI) Calcd. for $\mathrm{C}_{15} \mathrm{H}_{16} \mathrm{~F}_{2} \mathrm{O}_{5}:[\mathrm{M}]+\mathrm{Na}=337.0858$. Found: 337.0862 .<smiles>CCOC(=O)C=C(C(=O)OC)c1cccc(F)c1</smiles>

(E)-5-ethyl 1-methyl 4,4-difluoro-2-(3-fluorophenyl)pent-2-enedioate Yellow oil, $39.9 \mathrm{mg}, 66 \%$ yield, $\mathrm{E} / \mathrm{Z}=12: 1$.

${ }^{1} \mathbf{H}$ NMR (400 MHz, CDCl 3$) \delta$ ppm $7.37-7.31(\mathrm{~m}, 1 \mathrm{H}), 7.10-7.05$ (m, 2H), 6.98 (d, $J=$ $7.6 \mathrm{~Hz}, 1 \mathrm{H}), 6.93$ (d, $J=9.6 \mathrm{~Hz}, 1 \mathrm{H}), 4.04$ (q, $J=7.2 \mathrm{~Hz}, 2 \mathrm{H}), 3.81$ (s, 3H), 1.23 (t, $J=7.2$ $\mathrm{Hz}, 3 \mathrm{H})$.

${ }^{13}$ C NMR (100 MHz, CDCl $) \delta$ ppm 165.5, 162.2(t, $\left.J=32.8 \mathrm{~Hz}\right), 162.0(\mathrm{~d}, J=245.3 \mathrm{~Hz})$, $139.9(\mathrm{t}, J=8.0 \mathrm{~Hz}), 134.1(\mathrm{~d}, J=8.3 \mathrm{~Hz}), 132.3(\mathrm{t}, J=28.2 \mathrm{~Hz}), 129.5(\mathrm{~d}, J=8.3 \mathrm{~Hz})$, $125.2(\mathrm{~d}, J=2.9 \mathrm{~Hz}), 116.5(\mathrm{~d}, J=22.6 \mathrm{~Hz}), 115.9(\mathrm{~d}, J=20.9 \mathrm{~Hz}), 111.4(\mathrm{t}, J=246.8 \mathrm{~Hz})$, 63.3, 53.1, 13.6.

${ }^{19}$ F NMR (376 MHz, $\left.\mathbf{C D C l}_{3}\right) \delta$ ppm -95.7, -113.1.

IR (neat, $\mathrm{cm}^{-1}$ ): 3072, 2958, 1776, 1730, 1655, 1613, 1586, 1488, 1438, 1257, 1232, 1106, 1086, 1024, 789, 704.

HRMS (ESI) Calcd. for $\mathrm{C}_{14} \mathrm{H}_{13} \mathrm{~F}_{3} \mathrm{O}_{4}:[\mathrm{M}]+\mathrm{Na}=325.0658$. Found: 325.0662 . 
<smiles>CCOC(=O)CC=C(C(=O)OC)c1ccccc1OCC</smiles>

(E)-5-ethyl 1-methyl 4,4-difluoro-2-(2-methoxyphenyl)pent-2-enedioate Brown oil, $28.3 \mathrm{mg}, 45 \%$ yield, $\mathrm{E} / \mathrm{Z}>20: 1$.

${ }^{1}$ H NMR (400 MHz, CDCl $) \delta$ ppm $7.36(\mathrm{td}, J=7.6,2.0 \mathrm{~Hz}, 1 \mathrm{H}), 7.12(\mathrm{~d}, J=7.2 \mathrm{~Hz}, 1 \mathrm{H})$, $7.03-6.94(\mathrm{~m}, 2 \mathrm{H}), 6.88(\mathrm{~d}, J=8.0 \mathrm{~Hz}, 1 \mathrm{H}), 3.95(\mathrm{q}, J=7.2 \mathrm{~Hz}, 2 \mathrm{H}), 3.77$ (s, 3H), 3.76 (s, $3 \mathrm{H}), 1.19$ (t, $J=7.2 \mathrm{~Hz}, 3 \mathrm{H})$.

${ }^{13}$ C NMR (100 MHz, CDCl $) \delta$ ppm 166.2, $162.3(\mathrm{t}, J=33.0 \mathrm{~Hz}), 156.9,138.7(\mathrm{t}, J=8.1$ $\mathrm{Hz}), 131.4(\mathrm{t}, J=28.3 \mathrm{~Hz}), 131.0,130.7,121.7,120.1,111.6(\mathrm{t}, J=245.4 \mathrm{~Hz}), 110.4,63.0$, 55.5, 52.8, 13.6 .

${ }^{19}$ F NMR (376 MHz, CDCl 3$) \delta$ ppm -96.4.

IR $\left(\right.$ neat, $\left.\mathrm{cm}^{-1}\right): 3067,2955,1774,1730,1657,1601,1493,1464,1436,1256,1198,1104$, 1048, 1026, 757.

HRMS (ESI) Calcd. for $\mathrm{C}_{15} \mathrm{H}_{16} \mathrm{~F}_{2} \mathrm{O}_{5}:[\mathrm{M}]+\mathrm{Na}=337.0858$. Found: 337.0860 .<smiles>CCOC(=O)CC=C(C(=O)OC)c1ccccc1Cl</smiles>

\section{(E)-5-ethyl 1-methyl 2-(2-chlorophenyl)-4,4-difluoropent-2-enedioate}

Yellow oil, $40.0 \mathrm{mg}, 63 \%$ yield, E/Z=17:1.

${ }^{1} \mathbf{H}$ NMR (400 MHz, CDCl $) \delta$ ppm $7.40(\mathrm{~d}, J=6.8 \mathrm{~Hz}, 1 \mathrm{H}), 7.39-7.34(\mathrm{~m}, 1 \mathrm{H}), 7.32$ $7.26(\mathrm{~m}, 1 \mathrm{H}), 7.22-7.20(\mathrm{~m}, 1 \mathrm{H}), 7.14(\mathrm{t}, J=11.6 \mathrm{~Hz}, 1 \mathrm{H}), 4.07(\mathrm{~s}, 2 \mathrm{H}), 3.79(\mathrm{~s}, 3 \mathrm{H}), 1.25$ (t, $J=7.2 \mathrm{~Hz}, 3 \mathrm{H})$.

${ }^{13}$ C NMR (100 MHz, CDCl $) \delta$ ppm 165.0, $162.0(\mathrm{t}, J=32.8 \mathrm{~Hz}), 139.0(\mathrm{t}, J=7.9 \mathrm{~Hz})$, $133.3,132.7(\mathrm{t}, J=28.3 \mathrm{~Hz}), 131.6,131.3,130.4,129.0,126.4,111.3(\mathrm{t}, J=246.8 \mathrm{~Hz})$, 63.3, 53.1, 13.7 .

${ }^{19}$ F NMR (376 MHz, CDCl $) \delta$ ppm $-96.3(\mathrm{~d}, J=276.7 \mathrm{~Hz}),-98.5(\mathrm{~d}, J=281.6 \mathrm{~Hz})$.

IR (neat, $\left.\mathrm{cm}^{-1}\right): 3066,2957,1776,1732,1661,1474,1438,1253,1222,1110,1090,1016$, 767.

HRMS (ESI) Calcd. for $\mathrm{C}_{14} \mathrm{H}_{13} \mathrm{ClF}_{2} \mathrm{O}_{4}:[\mathrm{M}]+\mathrm{Na}=341.0363$. Found: 341.0368 .<smiles>CCOC(=O)CC=C(C(=O)OC)c1ccc(C(=O)c2ccccc2)cc1</smiles>

(E)-5-ethyl 1-methyl 2-(4-benzoylphenyl)-4,4-difluoropent-2-enedioate Brown oil, $36.6 \mathrm{mg}, 50 \%$ yield, E/Z>20:1.

${ }^{1}$ H NMR (400 MHz, CDCl $) \delta$ ppm $7.82-7.80(\mathrm{~m}, 4 \mathrm{H}), 7.60(\mathrm{t}, J=7.2 \mathrm{~Hz}, 1 \mathrm{H}), 7.50(\mathrm{t}, \quad J$ 
$=7.6 \mathrm{~Hz}, 2 \mathrm{H}), 7.33(\mathrm{~d}, J=8.4 \mathrm{~Hz}, 2 \mathrm{H}), 7.12(\mathrm{t}, J=12.0 \mathrm{~Hz}, 1 \mathrm{H}), 4.07(\mathrm{q}, J=7.2 \mathrm{~Hz}, 2 \mathrm{H})$, $3.83(\mathrm{~s}, 3 \mathrm{H}), 1.24(\mathrm{t}, J=7.2 \mathrm{~Hz}, 3 \mathrm{H})$.

${ }^{13}$ C NMR (100 MHz, CDCl $) \delta$ ppm 195.9, 165.5, $162.3(\mathrm{t}, J=32.8 \mathrm{~Hz}), 140.4(\mathrm{t}, J=7.7$ Hz), 137.7, 137.3, 136.4, 132.6, 132.2 (t, $J=27.8 \mathrm{~Hz}), 130.0,129.4,129.3,128.3,111.4 \quad$ (t, $J=247.2 \mathrm{~Hz}), 63.4,53.1,13.7$.

${ }^{19}$ F NMR (376 MHz, CDCl 3 ) $\delta$ ppm $-96.0(\mathrm{~d}, J=7.5 \mathrm{~Hz})$.

IR (neat, $\mathrm{cm}^{-1}$ ): 3062, 2956, 1774, 1729, 1661, 1606, 1488, 1448, 1437, 1255, 1212, 1108, 1085, 1014, 856, 737, 704.

HRMS (ESI) Calcd. for $\mathrm{C}_{21} \mathrm{H}_{18} \mathrm{~F}_{2} \mathrm{O}_{5}:[\mathrm{M}]+\mathrm{H}=389.1195$. Found: 389.1201 .<smiles>CCOC(=O)CC=C(C(=O)OC)c1ccc2cc(OC)ccc2c1</smiles>

(E)-5-ethyl 1-methyl 4,4-difluoro-2-(6-methoxynaphthalen-2-yl)pent-2-enedioate Brown oil, $48.0 \mathrm{mg}, 66 \%$ yield, $\mathrm{E} / \mathrm{Z}=17: 1$.

${ }^{1}$ H NMR (400 MHz, CDCl 3 ) $\delta$ ppm $7.72(\mathrm{t}, J=8.4 \mathrm{~Hz}, 2 \mathrm{H}), 7.60(\mathrm{~s}, 1 \mathrm{H}), 7.27-7.25(\mathrm{~m}$, $1 \mathrm{H}), 7.19-7.15(\mathrm{~m}, 1 \mathrm{H}), 7.13-7.08(\mathrm{~m}, 2 \mathrm{H}), 3.92(\mathrm{~s}, 3 \mathrm{H}), 3.81(\mathrm{~s}, 3 \mathrm{H}), 3.68(\mathrm{q}, J=7.2$ $\mathrm{Hz}, 2 \mathrm{H}), 0.98(\mathrm{t}, J=7.2 \mathrm{~Hz}, 3 \mathrm{H})$.

${ }^{13}$ C NMR (100 MHz, CDCl $) \delta$ ppm 166.2, $162.3(\mathrm{t}, J=32.9 \mathrm{~Hz}), 158.5,141.3(\mathrm{t}, J=9.0$ $\mathrm{Hz}), 134.5,131.8(\mathrm{t}, J=29.0 \mathrm{~Hz}), 129.8,129.1,127.9,127.3,127.2,126.2,119.3,111.7 \quad$ (t, $J=245.4 \mathrm{~Hz}), 105.7,63.0,55.3,53.0,13.4$.

${ }^{19}$ F NMR (376 MHz, CDCl 3 ) $\delta$ ppm -93.7.

IR (neat, $\left.\mathrm{cm}^{-1}\right): 3062,2956,1773,1727,1631,1605,1485,1464,1438,1260,1218,1105$, 1081, 1027, 855, 816,766, 700 .

HRMS (ESI) Calcd. for $\mathrm{C}_{19} \mathrm{H}_{18} \mathrm{~F}_{2} \mathrm{O}_{5}:[\mathrm{M}]+\mathrm{Na}=387.1015$. Found: 387.1019 .

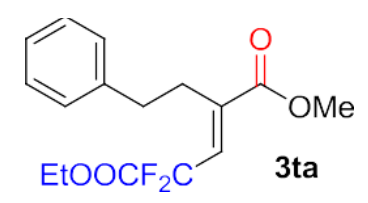

(E)-5-ethyl 1-methyl 4,4-difluoro-2-phenethylpent-2-enedioate Yellow oil, $28.8 \mathrm{mg}, 46 \%$ yield, E/Z=9:1.

${ }^{1}$ H NMR (400 MHz, CDCl $) \delta$ ppm $7.31-7.18(\mathrm{~m}, 5 \mathrm{H}), 6.76(\mathrm{t}, J=14.4 \mathrm{~Hz}, 1 \mathrm{H}), 4.33$ (q, $J=7.2 \mathrm{~Hz}, 2 \mathrm{H}), 3.79(\mathrm{~s}, 3 \mathrm{H}), 2.80-2.73(\mathrm{~m}, 4 \mathrm{H}), 1.34(\mathrm{t}, J=7.2 \mathrm{~Hz}, 3 \mathrm{H})$.

${ }^{13}$ C NMR (100 MHz, CDCl $) \delta$ ppm 166.5, $163.1(\mathrm{t}, J=33.6 \mathrm{~Hz}), 141.9(\mathrm{t}, J=5.8 \mathrm{~Hz})$, $140.9,129.7(\mathrm{t}, J=26.9 \mathrm{~Hz}), 128.4,128.4,126.2,113.5(\mathrm{t}, J=248.8 \mathrm{~Hz}), 63.5,52.6,35.6$, 30.2 , 13.9 .

${ }^{19}$ F NMR (376 MHz, CDCl 3$) \delta$ ppm $-100.2(\mathrm{~d}, J=11.3 \mathrm{~Hz})$.

IR $\left(\right.$ neat, $\mathrm{cm}^{-1}$ ): 3064, 2955, 1771, 1727, 1657, 1455, 1438, 1256, 1200, 1122, 1094, 1012, 745, 701.

HRMS (ESI) Calcd. for $\mathrm{C}_{16} \mathrm{H}_{18} \mathrm{~F}_{2} \mathrm{O}_{4}:[\mathrm{M}]+\mathrm{Na}=335.1065$. Found: 335.1068 . 
<smiles>CCOCCOC(=O)c1ccc(Cl)cc1</smiles>

(E)-5-ethyl 1-methyl 2-(2-((4-chlorobenzoyl)oxy)ethyl)-4,4-difluoropent-2-enedioate Yellow oil, $25.7 \mathrm{mg}, 33 \%$ yield, $E / Z=11: 1$.

${ }^{1}$ H NMR (400 MHz, CDCl $) \delta$ ppm 7.96 - $7.94(\mathrm{~m}, 2 \mathrm{H}), 7.42-7.39(\mathrm{~m}, 2 \mathrm{H}), 6.87(\mathrm{t}, J=$ $14.0 \mathrm{~Hz}, 1 \mathrm{H}), 4.46(\mathrm{t}, J=6.4 \mathrm{~Hz}, 2 \mathrm{H}), 4.32$ (q, $J=7.2 \mathrm{~Hz}, 2 \mathrm{H}), 3.78(\mathrm{~s}, 3 \mathrm{H}), 3.05-3.02$ (m, $2 \mathrm{H}), 1.34(\mathrm{t}, J=7.2 \mathrm{~Hz}, 3 \mathrm{H})$.

${ }^{13}$ C NMR (100 MHz, CDCl $) \delta$ ppm 166.1, 165.4, $162.9(\mathrm{t}, J=33.2 \mathrm{~Hz}), 139.4,138.3(\mathrm{t}, \quad J$ $=5.6 \mathrm{~Hz}), 131.5(\mathrm{t}, J=26.4 \mathrm{~Hz}), 131.0,128.6,128.4,112.0(\mathrm{t}, J=248.9 \mathrm{~Hz}), 63.6,63.3$, $52.8,27.3,13.8$.

${ }^{19}$ F NMR (376 MHz, CDCl $) \delta$ ppm $-100.1(\mathrm{~d}, J=11.3 \mathrm{~Hz})$.

IR (neat, $\left.\mathrm{cm}^{-1}\right): 3073,2956,1771,1727,1660,1596,1489,1439,1273,1209,1141,1095$, $1015,853,761$.

HRMS (ESI) Calcd. for $\mathrm{C}_{17} \mathrm{H}_{17} \mathrm{ClF}_{2} \mathrm{O}_{6}:[\mathrm{M}]+\mathrm{Na}=413.0574$. Found: 413.0580 .<smiles>COC(=O)/C(=C/C(F)(F)C(F)(F)C(F)(F)C(F)(F)F)c1ccccc1</smiles>

3va

methyl (E)-4,4,5,5,6,6,7,7,7-nonafluoro-2-phenylhept-2-enoate

Yellow oil, $22.8 \mathrm{mg}, 30 \%$ yield, E/Z>20:1.

${ }^{1}$ H NMR (400 MHz, CDCl 3 ) $\delta$ ppm $7.39-7.37(\mathrm{~m}, 3 \mathrm{H}), 7.22-7.20(\mathrm{~m}, 2 \mathrm{H}), 6.92(\mathrm{t}, J=$ $14.3 \mathrm{~Hz}, 1 \mathrm{H}), 3.80(\mathrm{~s}, 3 \mathrm{H})$.

${ }^{13}$ C NMR (100 MHz, CDCl $) \delta$ ppm $165.9,144.5(\mathrm{t}, J=4.3 \mathrm{~Hz}), 132.3,128.7,128.5(\mathrm{t}, J$ $=2.6 \mathrm{~Hz}), 127.78,124.8(\mathrm{t}, J=22.6 \mathrm{~Hz}), 53.3$

${ }^{19}$ F NMR (376 MHz, CDCl $) \delta$ ppm -81.02 - -81.09 (m, 3F), -107.12 (m, 2F), -123.81 $-123.89(\mathrm{~m}, 2 \mathrm{~F}),-125.78--125.86(\mathrm{~m}, 2 \mathrm{~F})$.

IR (neat, $\left.\mathrm{cm}^{-1}\right): 3064,2959,1784,1655,1438,1353,1236,1134,700$.

HRMS (ESI) Calcd. for $\mathrm{C}_{14} \mathrm{H}_{9} \mathrm{~F}_{9} \mathrm{O}_{2}:[\mathrm{M}]+\mathrm{H}=381.0532$. Found: 381.0530<smiles>CCOC(=O)CC=C(C(=O)OCC)c1ccccc1</smiles>

(E)-diethyl 4,4-difluoro-2-phenylpent-2-enedioate

Yellow oil, $41.7 \mathrm{mg}, 70 \%$ yield, E/Z>20:1.

${ }^{1} \mathbf{H}$ NMR (400 MHz, CDCl $) \delta$ ppm $\delta 7.37-7.34(\mathrm{~m}, 3 \mathrm{H}), 7.22-7.19(\mathrm{~m}, 2 \mathrm{H}), 7.04(\mathrm{t}, J=$ $11.4 \mathrm{~Hz}, 1 \mathrm{H}), 4.26(\mathrm{q}, J=7.2 \mathrm{~Hz}, 2 \mathrm{H}), 3.90(\mathrm{q}, J=7.2 \mathrm{~Hz}, 2 \mathrm{H}), 1.29(\mathrm{t}, J=7.2 \mathrm{~Hz}, 3 \mathrm{H})$, $1.16(\mathrm{t}, J=7.2 \mathrm{~Hz}, 3 \mathrm{H})$.

${ }^{13}$ C NMR (100 MHz, CDCl 3$) \delta$ ppm 165.51, $162.28(\mathrm{t}, J=33.0 \mathrm{~Hz}), 141.50,132.18$, 
$131.28(\mathrm{t}, J=28.9 \mathrm{~Hz}), 129.42,128.86,127.73,111.59(\mathrm{t}, J=246.9 \mathrm{~Hz}), 63.05,62.07$, $14.01,13.57$.

${ }^{19}$ F NMR (376 MHz, CDCl 3 ) $\delta$ ppm -94.4 (s).

IR (neat, $\mathrm{cm}^{-1}$ ): 3062, 2987, 1775, 1725, 1655, 1446, 1249, 1201, 1107, 1086, 1028, 774, 701.

HRMS (ESI) Calcd. for $\mathrm{C}_{15} \mathrm{H}_{16} \mathrm{~F}_{2} \mathrm{O}_{4}:[\mathrm{M}]+\mathrm{Na}=321.0909$. Found: 321.0912 .<smiles>CCCCOC(=O)/C(=C/C(F)(F)F)c1cccc(C(C)(C)C)c1</smiles>

(E)-1-butyl 5-ethyl 4,4-difluoro-2-phenylpent-2-enedioate

Yellow oil, $29.3 \mathrm{mg}, 45 \%$ yield, E/Z>20:1.

${ }^{1}$ H NMR (400 MHz, CDCl $) \delta$ ppm $7.37-7.33(\mathrm{~m}, 3 \mathrm{H}), 7.21-7.19(\mathrm{~m}, 2 \mathrm{H}), 7.03(\mathrm{t}, J=$ $11.2 \mathrm{~Hz}, 1 \mathrm{H}), 4.20(\mathrm{t}, J=6.4 \mathrm{~Hz}, 2 \mathrm{H}), 3.91(\mathrm{q}, J=7.2 \mathrm{~Hz}, 2 \mathrm{H}), 1.65-1.59(\mathrm{~m}, 2 \mathrm{H}), 1.38-$ $1.31(\mathrm{~m}, 2 \mathrm{H}), 1.17(\mathrm{t}, J=7.2 \mathrm{~Hz}, 3 \mathrm{H}), 0.91(\mathrm{t}, J=7.2 \mathrm{~Hz}, 3 \mathrm{H})$.

${ }^{13}$ C NMR (100 MHz, CDCl $) \delta$ ppm 165.6, $162.3(\mathrm{t}, J=32.5 \mathrm{~Hz}), 141.6(\mathrm{t}, J=8.8 \mathrm{~Hz})$, $132.3,131.2(\mathrm{t}, J=28.8 \mathrm{~Hz}), 129.4,128.8,127.7,112.8(\mathrm{~d}, J=245.5 \mathrm{~Hz}), 65.9,63.0,30.4$, 19.0, 13.6, 13.6 .

${ }^{19}$ F NMR (376 MHz, CDCl 3$) \delta$ ppm -94.4.

IR $\left(\right.$ neat, $\mathrm{cm}^{-1}$ ): 3062, 2962, 1775, 1725, 1655, 1446, 1249, 1196, 1108, 1086, 1018, 774, 700 .

HRMS (ESI) Calcd. for $\mathrm{C}_{17} \mathrm{H}_{20} \mathrm{~F}_{2} \mathrm{O}_{4}:[\mathrm{M}]+\mathrm{Na}=349.1222$. Found: 349.1227.<smiles>CCOCCOCCOC(=O)C=C(C(=O)OCC)c1ccccc1</smiles>

(E)-5-ethyl 1-isopropyl 4,4-difluoro-2-phenylpent-2-enedioate

Yellow oil, $25.6 \mathrm{mg}, 41 \%$ yield, $\mathrm{E} / \mathrm{Z}=14: 1$.

${ }^{1} \mathbf{H}$ NMR (400 MHz, CDCl 3$) \delta$ ppm $7.36-7.34(\mathrm{~m}, 3 \mathrm{H}), 7.21-7.19(\mathrm{~m}, 2 \mathrm{H}), 6.99(\mathrm{t}, J=$ $11.2 \mathrm{~Hz}, 1 \mathrm{H}), 5.15-5.06(\mathrm{~m}, 1 \mathrm{H}), 3.89$ (q, $J=7.2 \mathrm{~Hz}, 2 \mathrm{H}), 1.28$ (s, 3H), 1.27 (s, 4H), 1.16 (t, $J=7.2 \mathrm{~Hz}, 3 \mathrm{H})$.

${ }^{13}$ C NMR (100 MHz, CDCl $) \delta$ ppm 165.0, $162.4(\mathrm{t}, J=32.8 \mathrm{~Hz}), 141.9(\mathrm{t}, J=8.8 \mathrm{~Hz})$, $132.3,130.7(\mathrm{t}, J=28.8 \mathrm{~Hz}), 129.5,128.8,127.7,111.7(\mathrm{t}, J=245.6 \mathrm{~Hz}), 69.9,63.0,21.6$, 13.6.

${ }^{19}$ F NMR (376 MHz, CDCl 3$) \delta$ ppm -94.2.

IR (neat, $\mathrm{cm}^{-1}$ ): 3061, 2983, 1776, 1721, 1651, 1496, 1468, 1446, 1257, 1183, 1106, 1032, 774, 700 .

HRMS (ESI) Calcd. for $\mathrm{C}_{16} \mathrm{H}_{18} \mathrm{~F}_{2} \mathrm{O}_{4}:[\mathrm{M}]+\mathrm{Na}=335.1065$. Found: 335.1057. 
$\mathrm{EtOOCF}_{2} \mathrm{C}$

(E)-5-ethyl 1-isobutyl 4,4-difluoro-2-phenylpent-2-enedioate

Yellow oil, $28.7 \mathrm{mg}, 44 \%$ yield, $\mathrm{E} / \mathrm{Z}>20: 1$.

${ }^{1}$ H NMR (400 MHz, CDCl $) \delta$ ppm 7.37 - 7.35 (m, 3H), $7.21-7.19(\mathrm{~m}, 2 \mathrm{H}), 7.04(\mathrm{t}, J=$ $11.2 \mathrm{~Hz}, 1 \mathrm{H}), 3.98(\mathrm{~d}, J=6.4 \mathrm{~Hz}, 2 \mathrm{H}), 3.91$ (q, $J=7.2 \mathrm{~Hz}, 2 \mathrm{H}), 1.93$ (hept, $J=6.4 \mathrm{~Hz}, 1 \mathrm{H}$ ), $1.17(\mathrm{t}, J=7.2 \mathrm{~Hz}, 3 \mathrm{H}), 0.89$ (s, 3H), $0.87(\mathrm{~s}, 3 \mathrm{H})$.

${ }^{13}$ C NMR (100 MHz, CDCl $) \delta$ ppm 165.5, $162.3(\mathrm{t}, J=32.8 \mathrm{~Hz}), 141.6(\mathrm{t}, J=8.7 \mathrm{~Hz})$, 132.2, $131.3(\mathrm{t}, J=28.8 \mathrm{~Hz}), 129.4,128.8,127.7,111.6(\mathrm{t}, J=245.6 \mathrm{~Hz}), 72.0,63.1,27.6$, $18.9,13.6$.

${ }^{19}$ F NMR (376 MHz, CDCl 3$) \delta$ ppm -94.5.

IR (neat, $\mathrm{cm}^{-1}$ ): 3060, 2964, 1776, 1725, 1654, 1470, 1446, 1246, 1198, 1108, 1086, 1012 , 774,700 .

HRMS (ESI) Calcd. for $\mathrm{C}_{17} \mathrm{H}_{20} \mathrm{~F}_{2} \mathrm{O}_{4}:[\mathrm{M}]+\mathrm{Na}=349.1222$. Found: 349.1219 .<smiles>CCOCCCCOC(=O)C(=CC(=O)OCC)c1ccccc1</smiles>

(E)-1-cyclohexyl 5-ethyl 4,4-difluoro-2-phenylpent-2-enedioate Yellow oil, $24.6 \mathrm{mg}, 35 \%$ yield, E/Z>20:1.

${ }^{1}$ H NMR (400 MHz, CDCl $) \delta$ ppm $7.36-7.34(\mathrm{~m}, 3 \mathrm{H}), 7.22-7.19(\mathrm{~m}, 2 \mathrm{H}), 7.01(\mathrm{t}, J=$ $11.2 \mathrm{~Hz}, 1 \mathrm{H}), 4.89$ (tt, $J=8.4,3.6 \mathrm{~Hz}, 1 \mathrm{H}), 3.90(\mathrm{q}, J=7.2 \mathrm{~Hz}, 2 \mathrm{H}), 1.83-1.80(\mathrm{~m}, 2 \mathrm{H})$, $1.64-1.62(\mathrm{~m}, 2 \mathrm{H}), 1.51-1.47(\mathrm{~m}, 2 \mathrm{H}), 1.38-1.32(\mathrm{~m}, 2 \mathrm{H}), 1.29-1.26(\mathrm{~m}, 2 \mathrm{H}), 1.16 \quad(\mathrm{t}$, $J=7.2 \mathrm{~Hz}, 3 \mathrm{H})$.

${ }^{13}$ C NMR (100 MHz, CDCl $) \delta$ ppm 164.9, $162.4(\mathrm{t}, J=32.8 \mathrm{~Hz}), 142.0(\mathrm{t}, J=8.7 \mathrm{~Hz})$, 132.4, 130.9 (t, $J=28.8 \mathrm{~Hz}), 129.4,128.8,127.7,111.7(\mathrm{t}, J=245.4 \mathrm{~Hz}), 74.4,63.0,31.2$, 25.2, 23.3, 13.6 .

${ }^{19}$ F NMR (376 MHz, CDCl 3$) \delta$ ppm -94.2.

IR $\left(\right.$ neat, $\left.\mathrm{cm}^{-1}\right)$ : 3060, 2938, 1775, 1720, 1652, 1448, 1250, 1195, 1108, 1086, 1013, 774, 700.

HRMS (ESI) Calcd. for $\mathrm{C}_{19} \mathrm{H}_{22} \mathrm{~F}_{2} \mathrm{O}_{4}:[\mathrm{M}]+\mathrm{Na}=375.1378$. Found: 375.1382 .<smiles>CCOC(=O)CC=C(C(=O)OCc1ccccc1)c1ccccc1</smiles>

(E)-1-benzyl 5-ethyl 4,4-difluoro-2-phenylpent-2-enedioate

Yellow oil, $56.2 \mathrm{mg}, 78 \%$ yield, $\mathrm{E} / \mathrm{Z}=8: 1$.

${ }^{1}$ H NMR (400 MHz, CDCl $) \delta$ ppm 7.36 - 7.34 (m, 4H), 7.33 - 7.29 (m, 4H), 7.23 - 7.20 $(\mathrm{m}, 2 \mathrm{H}), 7.06(\mathrm{t}, J=11.2 \mathrm{~Hz}, 1 \mathrm{H}), 5.23(\mathrm{~s}, 2 \mathrm{H}), 3.89(\mathrm{q}, J=7.2 \mathrm{~Hz}, 2 \mathrm{H}), 1.14(\mathrm{t}, J=7.2 \mathrm{~Hz}$, 
$3 \mathrm{H})$.

${ }^{13}$ C NMR (100 MHz, CDCl $) \delta$ ppm 165.3, $162.2(\mathrm{t}, J=32.7 \mathrm{~Hz}), 141.2(\mathrm{t}, J=8.5 \mathrm{~Hz})$, $135.2,132.0,131.6(\mathrm{t}, J=28.8 \mathrm{~Hz}), 129.4,128.9,128.5,128.3,128.0,127.7,111.5(\mathrm{t}, J=$ $245.7 \mathrm{~Hz}), 67.5,63.0,13.5$.

${ }^{19}$ F NMR (376 MHz, CDCl $) \delta$ ppm $-94.4(\mathrm{~d}, J=3.8 \mathrm{~Hz})$.

IR (neat, $\mathrm{cm}^{-1}$ ): 3065, 2984, 1774, 1726, 1653, 1497, 1456, 1446, 1244, 1179, 1108, 1086, 1010, 773, 698 .

HRMS (ESI) Calcd. for $\mathrm{C}_{20} \mathrm{H}_{18} \mathrm{~F}_{2} \mathrm{O}_{4}$ : [M]+Na = 383.1065. Found: 383.1052 .<smiles>CCOC(=O)CC=C(C(=O)Oc1ccccc1)c1ccccc1</smiles>

(E)-5-ethyl 1-phenyl 4,4-difluoro-2-phenylpent-2-enedioate Yellow oil, $27.7 \mathrm{mg}, 40 \%$ yield, E/Z=15:1.

${ }^{1}$ H NMR (400 MHz, CDCl 3$) \delta$ ppm 7.40 - $7.36(\mathrm{~m}, 5 \mathrm{H}), 7.33$ - 7.30 (m, 2H), 7.28 - 7.24 $(\mathrm{m}, 1 \mathrm{H}), 7.25(\mathrm{t}, J=11.2 \mathrm{~Hz}, 1 \mathrm{H}), 7.12(\mathrm{~d}, J=7.8 \mathrm{~Hz}, 2 \mathrm{H}), 3.94(\mathrm{q}, J=7.2 \mathrm{~Hz}, 2 \mathrm{H}), 1.19$ (t, $J=7.2 \mathrm{~Hz}, 3 \mathrm{H})$.

${ }^{13}$ C NMR (100 MHz, $\left.\mathbf{C C l}_{3}\right) \delta$ ppm 164.1, $162.2(\mathrm{t}, J=32.7 \mathrm{~Hz}), 150.5,141.0(\mathrm{t}, J=8.6$ $\mathrm{Hz}), 132.8(\mathrm{t}, J=28.9 \mathrm{~Hz}), 131.8,129.5,129.5,129.1,127.9,126.2,121.2,111.5(\mathrm{t}, J=$ $246.0 \mathrm{~Hz}), 63.2,13.6$.

${ }^{19}$ F NMR (376 MHz, CDCl 3$) \delta$ ppm $-94.5(\mathrm{~d}, J=3.8 \mathrm{~Hz})$.

IR (neat, $\mathrm{cm}^{-1}$ ): 3062, 2985, 1775, 1741, 1653, 1592, 1492, 1446, 1227, 1191, 1107, 1084, $1029,773,743,699,789$.

HRMS (ESI) Calcd. for $\mathrm{C}_{19} \mathrm{H}_{16} \mathrm{~F}_{2} \mathrm{O}_{4}:[\mathrm{M}]+\mathrm{Na}=369.0909$. Found: 369.0912 .<smiles>CCOC(F)(F)F</smiles>

(E)-5-ethyl 1-(4-methoxyphenyl) 4,4-difluoro-2-phenylpent-2-enedioate Brown oil, $39.9 \mathrm{mg}, 53 \%$ yield, E/Z>20:1.

${ }^{1}$ H NMR (400 MHz, CDCl $) \delta$ ppm $7.40-7.38(\mathrm{~m}, 3 \mathrm{H}), 7.32-7.29$ (m, 2H), $7.23(\mathrm{t}, \mathrm{J}=$ $11.2 \mathrm{~Hz}, 1 \mathrm{H}), 7.04(\mathrm{~d}, \mathrm{~J}=9.1 \mathrm{~Hz}, 2 \mathrm{H}), 6.88(\mathrm{~d}, \mathrm{~J}=9.1 \mathrm{~Hz}, 2 \mathrm{H}), 3.94(\mathrm{q}, \mathrm{J}=7.2 \mathrm{~Hz}, 2 \mathrm{H})$, $3.78(\mathrm{~s}, 3 \mathrm{H}), 1.18(\mathrm{t}, \mathrm{J}=7.2 \mathrm{~Hz}, 3 \mathrm{H})$.

${ }^{13}$ C NMR (100 MHz, CDCl $) \delta$ ppm 164.5, $162.2(\mathrm{t}, J=32.7 \mathrm{~Hz}), 157.5,144.0,141.0(\mathrm{t}, \quad J$ $=8.6 \mathrm{~Hz}), 132.7(\mathrm{t}, J=28.8 \mathrm{~Hz}), 131.8,129.5,129.1,127.9,122.0,114.4,112.7(\mathrm{t}, J=$ $246.0 \mathrm{~Hz}), 63.2,55.5,13.6$.

${ }^{19}$ F NMR (376 MHz, CDCl $) \delta$ ppm $-94.4(\mathrm{~d}, J=7.5 \mathrm{~Hz})$.

IR (neat, $\mathrm{cm}^{-1}$ ): 3063, 2934, 1774, 1736, 1654, 1507, 1463, 1445, 1224, 1193, 1106, 1087, 1031, 857, 775, 701.

HRMS (ESI) Calcd. for $\mathrm{C}_{20} \mathrm{H}_{18} \mathrm{~F}_{2} \mathrm{O}_{5}:[\mathrm{M}]+\mathrm{Na}=399.1015$. Found: 399.1020 . 
<smiles>CCOC(F)(F)F</smiles>

(E)-1-(4-chlorophenyl) 5-ethyl 4,4-difluoro-2-phenylpent-2-enedioate Yellow oil, $33.4 \mathrm{mg}, 44 \%$ yield, $\mathrm{E} / \mathrm{Z}=11: 1$.

${ }^{1} \mathbf{H}$ NMR (400 MHz, CDCl $) \delta$ ppm 7.41 - $7.39(\mathrm{~m}, 3 \mathrm{H}), 7.35(\mathrm{~d}, J=8.8 \mathrm{~Hz}, 2 \mathrm{H}), 7.31$ $7.28(\mathrm{~m}, 2 \mathrm{H}), 7.24(\mathrm{t}, J=11.2 \mathrm{~Hz}, 1 \mathrm{H}), 7.07(\mathrm{~d}, J=8.8 \mathrm{~Hz}, 2 \mathrm{H}), 3.95(\mathrm{q}, J=7.2 \mathrm{~Hz}, 2 \mathrm{H})$, $1.19(\mathrm{t}, J=7.2 \mathrm{~Hz}, 3 \mathrm{H})$.

${ }^{13}$ C NMR (100 MHz, CDCl $) \delta 163.9,162.1(\mathrm{t}, J=32.5 \mathrm{~Hz}), 148.9,140.6(\mathrm{t}, J=8.4 \mathrm{~Hz})$, $133.3(\mathrm{t}, J=28.9 \mathrm{~Hz}), 131.6,131.6,129.5,129.5,129.3,128.0,122.6,111.4(\mathrm{t}, J=246.3$ $\mathrm{Hz}), 63.3,13.6$.

${ }^{19}$ F NMR (376 MHz, CDCl 3 ) $\delta$ ppm -94.5.

IR $\left(\right.$ neat, $\left.\mathrm{cm}^{-1}\right): 3065,2987,1775,1743,1655,1487,1446,1194,1089,1014,856, \quad 774$, 700.

HRMS (ESI) Calcd. for $\mathrm{C}_{19} \mathrm{H}_{15} \mathrm{ClF}_{2} \mathrm{O}_{4}:[\mathrm{M}]+\mathrm{Na}=403.0519$. Found: 403.0523.<smiles>CCOC(=O)CC=C(C(=O)Oc1ccc(C(C)(C)C)cc1)c1cccc(Br)c1</smiles>

(E)-1-(4-(tert-butyl)phenyl) 5-ethyl 4,4-difluoro-2-phenylpent-2-enedioate Yellow oil, $41.0 \mathrm{mg}, 51 \%$ yield, E/Z>20:1.

${ }^{1} \mathbf{H}$ NMR (400 MHz, CDCl$\left.)_{3}\right) \delta 7.39-7.37(\mathrm{~m}, 5 \mathrm{H}), 7.32-7.31(\mathrm{~m}, 2 \mathrm{H}), 7.23(\mathrm{t}, J=11.2$ $\mathrm{Hz}, 1 \mathrm{H}), 7.04(\mathrm{~d}, J=8.8 \mathrm{~Hz}, 2 \mathrm{H}), 3.94(\mathrm{q}, J=7.2 \mathrm{~Hz}, 2 \mathrm{H}), 1.31(\mathrm{~s}, 9 \mathrm{H}), 1.19(\mathrm{t}, J=7.2 \mathrm{~Hz}$, $3 \mathrm{H})$.

${ }^{13}$ C NMR (100 MHz, CDCl $) \delta$ ppm 164.3, $162.2(\mathrm{t}, J=32.7 \mathrm{~Hz}), 149.1,148.2,141.1(\mathrm{t}, \quad J$ $=8.5 \mathrm{~Hz}), 132.7(\mathrm{t}, J=28.8 \mathrm{~Hz}), 131.9,129.5,129.1,127.9,126.3,120.5,111.5(\mathrm{t}, J=$ 245.8 Hz), 63.2, 34.5, 31.4, 13.6 .

${ }^{19}$ F NMR (376 MHz, CDCl 3$) \delta$ ppm $-94.4(\mathrm{~d}, J=7.5 \mathrm{~Hz})$.

IR $\left(\right.$ neat, $\mathrm{cm}^{-1}$ ): 3061, 2871, 1775, 1736, 1654, 1510, 1462, 1446, 1198, 1175, 1107, 1083, 1016, 858, 774, 699.

HRMS (ESI) Calcd. for $\mathrm{C}_{23} \mathrm{H}_{24} \mathrm{~F}_{2} \mathrm{O}_{4}:[\mathrm{M}]+\mathrm{Na}=425.1535$. Found: 425.1540 .<smiles>CCOC(F)(F)C(F)(F)F</smiles>

(E)-ethyl 2,2-difluoro-5-oxo-4-phenyl-5-((phenylamino)oxy)pent-3-enoate.

Brown oil, $49.7 \mathrm{mg}, 72 \%$ yield, only E.

${ }^{1}$ H NMR (400 MHz, CDCl $) \delta$ ppm $7.49-7.48$ (m, 3H), $7.41-7.38$ (m, 2H), 7.33 - 7.27 $(\mathrm{m}, 4 \mathrm{H}), 7.22-7.16(\mathrm{~m}, 2 \mathrm{H}), 7.11(\mathrm{t}, J=7.2 \mathrm{~Hz}, 1 \mathrm{H}), 4.04(\mathrm{q}, J=7.2 \mathrm{~Hz}, 2 \mathrm{H}), 1.23(\mathrm{t}, J=$ $7.2 \mathrm{~Hz}, 3 \mathrm{H})$. 
${ }^{13}$ C NMR (100 MHz, CDCl $) \delta$ ppm $162.5(\mathrm{t}, J=33.1 \mathrm{~Hz}), 162.3,143.4(\mathrm{t}, J=7.6 \mathrm{~Hz})$, $136.9,132.0,129.7,129.3,129.3(\mathrm{t}, J=27.6 \mathrm{~Hz}), 129.0,128.9,125.1,120.0,111.9(\mathrm{t}, J=$ $246.4 \mathrm{~Hz}), 63.1,13.7$.

${ }^{19}$ F NMR (376 MHz, CDCl 3 ) $\delta$ ppm -96.1.

IR (neat, $\mathrm{cm}^{-1}$ ): 3401, 3305, 3060, 2987, 1774, 1687, 1600, 1541, 1498, 1442, 1395, 1306, 1250, 1201, 1108, 1031, 756, 699.

HRMS (ESI) Calcd. for $\mathrm{C}_{19} \mathrm{H}_{17} \mathrm{~F}_{2} \mathrm{NO}_{3}:[\mathrm{M}]+\mathrm{H}=346.1253$. Found: 346.1249 .<smiles>CCOC(=O)C=C(C(=O)Nc1ccc(Br)cc1)c1ccccc1</smiles>

(E)-ethyl 5-((4-bromophenyl)amino)-2,2-difluoro-5-oxo-4-phenylpent-3-enoate Brown oil, $37.2 \mathrm{mg}, 45 \%$ yield, $\mathrm{E} / \mathrm{Z}>20: 1$.

${ }^{1} \mathbf{H}$ NMR (400 MHz, CDCl$) \delta$ ppm $7.50-7.49(\mathrm{~m}, 3 \mathrm{H}), 7.40(\mathrm{~d}, J=8.8 \mathrm{~Hz}, 2 \mathrm{H}), 7.32$ $7.29(\mathrm{~m}, 3 \mathrm{H}), 7.23-7.16(\mathrm{~m}, 2 \mathrm{H}), 6.55(\mathrm{~d}, J=8.6 \mathrm{~Hz}, 1 \mathrm{H}), 4.05(\mathrm{q}, J=7.2 \mathrm{~Hz}, 2 \mathrm{H}), 1.24$ (t, $J=7.2 \mathrm{~Hz}, 3 \mathrm{H})$.

${ }^{13}$ C NMR (100 MHz, CDCl $) \delta$ ppm $162.5(\mathrm{t}, J=32.8 \mathrm{~Hz}), 162.3,143.0(\mathrm{t}, J=7.6 \mathrm{~Hz})$, $136.0,132.0,129.9(\mathrm{t}, J=27.6 \mathrm{~Hz}), 129.8,129.3,129.0,121.5,117.9,116.7,111.8(\mathrm{t}, J=$ $246.9 \mathrm{~Hz}), 63.2,13.7$.

${ }^{19}$ F NMR (376 MHz, CDCl $) \delta$ ppm $-96.3(\mathrm{~d}, J=7.5 \mathrm{~Hz})$.

IR (neat, $\mathrm{cm}^{-1}$ ): 3395, 3310, 3059, 2983, 1771, 1684, 1592, 1519, 1490, 1445, 1395, 1306, 1236, 1212, 1107, 1084, 1010, 820, 773, 701 .

HRMS (ESI) Calcd. for $\mathrm{C}_{19} \mathrm{H}_{16} \mathrm{BrF}_{2} \mathrm{NO}_{3}:[\mathrm{M}]+\mathrm{Na}=446.0714$. Found: 446.0180 .<smiles>CCOC(=O)CC=C(C(=O)Nc1ccc(C(C)(C)C)cc1)c1ccccc1</smiles>

(E)-ethyl 5-((4-(tert-butyl)phenyl)amino)-2,2-difluoro-5-oxo-4-phenylpent-3-enoate Brown oil, $56.1 \mathrm{mg}, 70 \%$ yield, $\mathrm{E} / \mathrm{Z}>20: 1$.

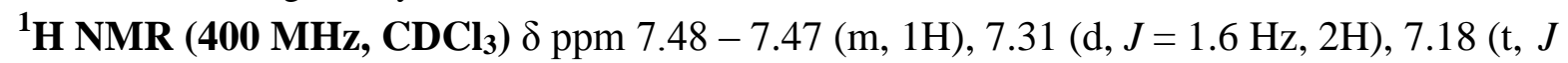
$=12.2 \mathrm{~Hz}, 1 \mathrm{H}), 7.17(\mathrm{~s}, 1 \mathrm{H}), 4.03(\mathrm{q}, J=7.2 \mathrm{~Hz}, 1 \mathrm{H}), 1.28(\mathrm{~s}, 3 \mathrm{H}), 1.22(\mathrm{t}, J=7.2 \mathrm{~Hz}, 1 \mathrm{H})$.

${ }^{13}$ C NMR (100 MHz, CDCl 3 ) $\delta$ ppm $162.5(\mathrm{t}, J=33.0 \mathrm{~Hz}), 162.3,148.2,143.5(\mathrm{t}, J=7.6$ $\mathrm{Hz}), 134.3,132.1,129.6,129.3,129.2$ (t, $J=27.6 \mathrm{~Hz}), 128.9,125.8,119.8,111.9(\mathrm{t}, J=$ $246.3 \mathrm{~Hz}), 63.1,34.4,31.2,13.7$.

${ }^{19}$ F NMR (376 MHz, CDCl 3$) \delta$ ppm $-95.96(\mathrm{~d}, J=7.5 \mathrm{~Hz})$.

IR (neat, $\mathrm{cm}^{-1}$ ): 3404, 3305, 3059, 2962, 1773, 1725, 1685, 1644, 1595, 1521, 1475, 1445, 1407, 1215, 1201, 1108, 1084, 1031, 834, 773, 701.

HRMS (ESI) Calcd. for $\mathrm{C}_{23} \mathrm{H}_{25} \mathrm{~F}_{2} \mathrm{NO}_{3}:[\mathrm{M}]+\mathrm{Na}=424.1695$. Found: 424.1700 .<smiles>CCOC(=O)C(=CC(=O)Nc1ccccc1OC)c1ccccc1</smiles>

(E)-ethyl 2,2-difluoro-5-((2-methoxyphenyl)amino)-5-oxo-4-phenylpent-3-enoate 
Brown oil, $28.2 \mathrm{mg}, 40 \%$ yield, $\mathrm{E} / \mathrm{Z}=12: 1$.

${ }^{1}$ H NMR (400 MHz, CDCl $) \delta$ ppm $8.42-8.40(\mathrm{~m}, 1 \mathrm{H}), 8.01(\mathrm{~s}, 1 \mathrm{H}), 7.49-7.48(\mathrm{~m}, 3 \mathrm{H})$, $7.33-7.31(\mathrm{~m}, 2 \mathrm{H}), 7.20(\mathrm{t}, J=12.2 \mathrm{~Hz}, 1 \mathrm{H}), 7.05-7.01(\mathrm{~m}, 1 \mathrm{H}), 6.96(\mathrm{t}, J=7.4 \mathrm{~Hz}, 1 \mathrm{H})$, $6.76(\mathrm{~d}, J=8.0 \mathrm{~Hz}, 1 \mathrm{H}), 4.04(\mathrm{q}, J=7.2 \mathrm{~Hz}, 2 \mathrm{H}), 3.58(\mathrm{~s}, 3 \mathrm{H}), 1.23(\mathrm{t}, J=7.2 \mathrm{~Hz}, 3 \mathrm{H})$.

${ }^{13}$ C NMR (100 MHz, CDCl $) \delta$ ppm $162.6(\mathrm{t}, J=33.4 \mathrm{~Hz}), 161.9,148.1,143.8(\mathrm{t}, J=7.6$ $\mathrm{Hz}), 132.2,129.4,129.3,128.9(\mathrm{t}, J=27.6 \mathrm{~Hz}), 128.6,128.6,127.4,127.0,124.5,112.0(\mathrm{t}, J$ $=246.3 \mathrm{~Hz}), 109.9,63.1,55.6,13.7$.

${ }^{19}$ F NMR (376 MHz, CDCl $) \delta$ ppm $-96.2(\mathrm{~d}, J=7.5 \mathrm{~Hz})$.

IR (neat, $\mathrm{cm}^{-1}$ ): 3384, 3060, 2984, 1773, 1688, 1649, 1602, 1529, 1485, 1448, 1463, 1436, 1253, 1223, 1108, 1083, 1029, 858, 752, 706 .

HRMS (ESI) Calcd. for $\mathrm{C}_{20} \mathrm{H}_{19} \mathrm{~F}_{2} \mathrm{NO}_{4}:[\mathrm{M}]+\mathrm{Na}=376.1355$. Found: 376.1360 .<smiles>CCOC(F)(F)C(=O)N(CC)CC</smiles>

(E)-ethyl 5-(diethylamino)-2,2-difluoro-5-oxo-4-phenylpent-3-enoate Brown oil, $44.8 \mathrm{mg}, 74 \%$ yield, only $\mathrm{E}$.

${ }^{1}$ H NMR (400 MHz, CDCl $) \delta$ ppm $7.42-7.41(\mathrm{~m}, 2 \mathrm{H}), 7.37-7.35(\mathrm{~m}, 3 \mathrm{H}), 5.96(\mathrm{t}, J=$ $11.6 \mathrm{~Hz}, 1 \mathrm{H}), 3.96(\mathrm{q}, J=7.2 \mathrm{~Hz}, 2 \mathrm{H}), 3.40(\mathrm{q}, J=7.2 \mathrm{~Hz}, 2 \mathrm{H}), 3.32(\mathrm{q}, J=7.2 \mathrm{~Hz}, 2 \mathrm{H})$, $1.13(\mathrm{td}, J=7.2,2.4 \mathrm{~Hz}, 6 \mathrm{H}), 0.95(\mathrm{t}, J=7.2 \mathrm{~Hz}, 3 \mathrm{H})$.

${ }^{13}$ C NMR (100 MHz, CDCl $) \delta$ ppm 168.1, $162.7(\mathrm{t}, J=33.4 \mathrm{~Hz}), 147.1(\mathrm{t}, J=8.7 \mathrm{~Hz})$, $132.9,129.2,128.3,128.2,120.4(\mathrm{t}, J=28.2 \mathrm{~Hz}), 111.8(\mathrm{t}, J=245.0 \mathrm{~Hz}), 62.9,42.7,39.1$, $13.6,13.5,12.5$.

${ }^{19}$ F NMR (376 MHz, CDCl 3 ) $\delta$ ppm $-92.9(\mathrm{~d}, J=7.5 \mathrm{~Hz})$.

IR (neat, $\left.\mathrm{cm}^{-1}\right)$ : 3059, 2981, 1773, 1729, 1636, 1474, 1443, 1382, 1308, 1278, 1218, 1186, 1006, 1086, 1034, 774, 701.

HRMS (ESI) Calcd. for $\mathrm{C}_{17} \mathrm{H}_{21} \mathrm{~F}_{2} \mathrm{NO}_{3}:[\mathrm{M}]+\mathrm{Na}=326.1562$. Found: 326.1567.<smiles>CCOC(=O)C1CCN(C(=O)C(=CC(C)(C)OCC)c2ccccc2)CC1</smiles>

(E)-ethyl 1-(5-ethoxy-4,4-difluoro-5-oxo-2-phenylpent-2-enoyl)piperidine-4-carboxylate. Brown oil, $48.3 \mathrm{mg}, 59 \%$ yield, only E.

${ }^{1}$ H NMR (400 MHz, CDCl 3 ) $\delta$ ppm $7.39-7.35(\mathrm{~m}, 5 \mathrm{H}), 5.97(\mathrm{t}, J=11.6 \mathrm{~Hz}, 1 \mathrm{H}), 4.37$ $4.33(\mathrm{~m}, 1 \mathrm{H}), 4.13(\mathrm{q}, J=7.2 \mathrm{~Hz}, 2 \mathrm{H}), 3.96(\mathrm{q}, J=7.2 \mathrm{~Hz}, 2 \mathrm{H}), 3.88(\mathrm{~d}, J=13.6 \mathrm{~Hz}, 1 \mathrm{H})$, $3.11(\mathrm{t}, J=13.6 \mathrm{~Hz}, 1 \mathrm{H}), 2.93(\mathrm{t}, J=13.2 \mathrm{~Hz}, 1 \mathrm{H}), 2.53-2.48(\mathrm{~m}, 1 \mathrm{H}), 1.95(\mathrm{~d}, J=11.2$ $\mathrm{Hz}, 1 \mathrm{H}), 1.78(\mathrm{~d}, J=10.0 \mathrm{~Hz}, 1 \mathrm{H}), 1.64(\mathrm{~d}, J=10.0 \mathrm{~Hz}, 1 \mathrm{H}), 1.42(\mathrm{~d}, J=9.6 \mathrm{~Hz}, 1 \mathrm{H}), 1.24$ (t, $J=7.2 \mathrm{~Hz}, 3 \mathrm{H}), 1.14(\mathrm{t}, J=7.2 \mathrm{~Hz}, 3 \mathrm{H})$.

${ }^{13}$ C NMR (100 MHz, CDCl $) \delta$ ppm 173.8, 167.2, $162.7(\mathrm{t}, J=33.2 \mathrm{~Hz}), 146.3(\mathrm{t}, J=8.7$ $\mathrm{Hz}), 132.8,129.3,128.4,128.2,121.0(\mathrm{t}, J=28.2 \mathrm{~Hz}), 111.7$ (t, $J=245.3 \mathrm{~Hz}), 63.0,60.6$, 46.0, 40.9, 40.7, 28.0, 27.5, 14.1, 13.6.

${ }^{19}$ F NMR (376 MHz, CDCl $) \delta$ ppm -93.1. 
IR (neat, $\left.\mathrm{cm}^{-1}\right): 3059,2985,1774,1728,1687,1639,1445,1306,1251,1199,1107, \quad 1084$, 1041, 857, 776, 701.

HRMS (ESI) Calcd. for $\mathrm{C}_{21} \mathrm{H}_{25} \mathrm{~F}_{2} \mathrm{NO}_{5}:[\mathrm{M}]+\mathrm{H}=410.1774$. Found: 410.1778 .<smiles>O=C(C=C(C(=O)N1CCCCC1)c1ccccc1)N1CCCCC1</smiles>

(E)-4,4-difluoro-2-phenyl-1,5-di(piperidin-1-yl)pent-2-ene-1,5-dione Brown oil, $36.1 \mathrm{mg}, 48 \%$ yield, $\mathrm{E} / \mathrm{Z}>20: 1$.

${ }^{1}$ H NMR (400 MHz, CDCl $) \delta$ ppm $7.47-7.44(\mathrm{~m}, 2 \mathrm{H}), 7.35-7.34(\mathrm{~m}, 3 \mathrm{H}), 6.00(\mathrm{t}, J=$ $11.6 \mathrm{~Hz}, 1 \mathrm{H}), 3.57-3.54(\mathrm{~m}, 2 \mathrm{H}), 3.41-3.38(\mathrm{~m}, 4 \mathrm{H}), 3.24-3.21(\mathrm{~m}, 2 \mathrm{H}), 1.56-1.55(\mathrm{~m}$, $8 \mathrm{H}), 1.39-1.38(\mathrm{~m}, 2 \mathrm{H}), 1.29-1.26(\mathrm{~m}, 2 \mathrm{H})$.

${ }^{13}$ C NMR (100 MHz, CDCl $) \delta$ ppm 167.3, $160.4(\mathrm{t}, J=29.0 \mathrm{~Hz}), 145.0(\mathrm{t}, J=8.6 \mathrm{~Hz})$, 132.7, 129.1, 128.3, 128.1, $121.8(\mathrm{t}, J=28.0 \mathrm{~Hz}), 114.0(\mathrm{t}, J=245.7 \mathrm{~Hz}), 47.8,47.0,43.9$, 42.6, 25.8, 25.8, 25.3, 25.1, 24.3, 24.2.

${ }^{19}$ F NMR (376 MHz, CDCl 3$) \delta$ ppm -88.7.

IR (neat, $\mathrm{cm}^{-1}$ ): 3057, 2937, 2859, 1677, 1656, 1637, 1444, 1258, 1210, 1106, 1094, 1033, 758, 734, 701 .

HRMS (ESI) Calcd. for $\mathrm{C}_{21} \mathrm{H}_{26} \mathrm{~F}_{2} \mathrm{~N}_{2} \mathrm{O}_{2}:[\mathrm{M}]+\mathrm{H}=377.2035$. Found: 377.2028.<smiles>O=C(CC=C(C(=O)NC1CCCCC1)c1ccccc1)NC1CCCCC1</smiles>

\section{(E)- $\mathbf{N}^{\mathbf{1}}, \mathbf{N}^{\mathbf{5}}$-dicyclohexyl-4,4-difluoro-2-phenylpent-2-enediamide} white solid, $47.7 \mathrm{mg}, 59 \%$ yield, $\mathrm{E} / \mathrm{Z}>20: 1$.

${ }^{1}$ H NMR (400 MHz, CDCl $) \delta$ ppm $7.41(\mathrm{~d}, J=5.2 \mathrm{~Hz}, 3 \mathrm{H}), 7.27(\mathrm{~d}, J=4.8 \mathrm{~Hz}, 2 \mathrm{H}), 7.06$ $(\mathrm{t}, J=13.2 \mathrm{~Hz}, 1 \mathrm{H}), 5.90(\mathrm{~d}, J=8.0 \mathrm{~Hz}, 1 \mathrm{H}), 5.34(\mathrm{~d}, J=8.4 \mathrm{~Hz}, 1 \mathrm{H}), 3.81-3.79(\mathrm{~m}, 1 \mathrm{H})$, $3.57-3.45(\mathrm{~m}, 1 \mathrm{H}), 1.79(\mathrm{t}, J=12.8 \mathrm{~Hz}, 4 \mathrm{H}), 1.68-1.56(\mathrm{~m}, 6 \mathrm{H}), 1.32-1.26(\mathrm{~m}, 5 \mathrm{H})$, $1.14-0.98(\mathrm{~m}, 5 \mathrm{H})$.

${ }^{13}$ C NMR (100 MHz, CDCl $) \delta$ ppm 163.6, $162.0(\mathrm{t}, J=29.0 \mathrm{~Hz}), 143.1(\mathrm{t}, J=7.0 \mathrm{~Hz})$, 133.1, 129.2, 128.9, $128.5(\mathrm{t}, J=26.9 \mathrm{~Hz}), 128.4,113.6(\mathrm{t}, J=249.5 \mathrm{~Hz}), 48.7,48.5,32.4$, 32.3, 25.3, 25.2, 24.5, 24.4.

${ }^{19}$ F NMR (376 MHz, CDCl 3$) \delta$ ppm -97.0.

IR (neat, $\mathrm{cm}^{-1}$ ): 3338, 3254, 3064, 2927, 1688, 1634, 1653, 1539, 1451, 1243, 1230, 1122, $1088,844$.

HRMS (ESI) Calcd. for $\mathrm{C}_{23} \mathrm{H}_{30} \mathrm{~F}_{2} \mathrm{~N}_{2} \mathrm{O}_{3}:[\mathrm{M}]+\mathrm{Na}=427.2168$. Found: 427.2164.<smiles>CCOC(F)(F)F</smiles>

${ }^{1}$ H NMR (400 MHz, CDCl 3$) \delta$ ppm major: 7.82 - $7.79(\mathrm{~m}, 2 \mathrm{H}), 7.60-7.56(\mathrm{~m}, 1 \mathrm{H}), 7.37$ 
$-7.16(\mathrm{~m}, 7 \mathrm{H}), 6.27(\mathrm{t}, J=11.6 \mathrm{~Hz}, 1 \mathrm{H}), 3.90(\mathrm{q}, J=7.2 \mathrm{~Hz}, 2 \mathrm{H}), 1.16(\mathrm{t}, J=7.2 \mathrm{~Hz}, 3 \mathrm{H})$. minor: $7.49-7.46(\mathrm{~m}, 2 \mathrm{H}), 7.32-7.23(\mathrm{~m}, 8 \mathrm{H}), 4.27(\mathrm{t}, J=7.2 \mathrm{~Hz}, 1 \mathrm{H}), 3.83(\mathrm{q}, J=7.2$ $\mathrm{Hz}, 2 \mathrm{H}), 2.93(\mathrm{td}, J=15.2,7.2 \mathrm{~Hz}, 2 \mathrm{H}), 1.15(\mathrm{t}, J=7.2 \mathrm{~Hz}, 3 \mathrm{H})$.

${ }^{13}$ C NMR (100 MHz, CDCl $) \delta$ ppm major: $163.7(\mathrm{t}, J=32.5 \mathrm{~Hz}), 151.0(\mathrm{t}, J=9.5 \mathrm{~Hz})$, $142.9,140.5,132.34,129.8,129.1,128.6,128.2,127.7,119.5(\mathrm{t}, J=28.2 \mathrm{~Hz}), 112.5(\mathrm{t}, J=$ $243.6 \mathrm{~Hz}), 62.7,13.6$. minor: $163.4(\mathrm{t}, J=33.5 \mathrm{~Hz}), 137.6,137.1,132.4,130.0,128.4$, 128.0, 127.9, 126.7, $115.6(\mathrm{t}, J=249.1 \mathrm{~Hz}), 62.6,44.8(\mathrm{t}, J=4.7 \mathrm{~Hz}), 40.2(\mathrm{t}, J=23.1 \mathrm{~Hz})$, 13.6.

${ }^{19}$ F NMR (376 MHz, CDCl $) \delta$ ppm major: -91.0 (d, $\left.J=11.3 \mathrm{~Hz}, 2 \mathrm{~F}\right)$.

minor: $-103.4(\mathrm{t}, J=15.0 \mathrm{~Hz}, 2 \mathrm{~F})$

IR (neat, $\mathrm{cm}^{-1}$ ): 3303, 2984, 1768, 1660, 1279, 1069, 739.

HRMS (ESI) Calcd. for $\mathrm{C}_{18} \mathrm{H}_{16} \mathrm{~F}_{2} \mathrm{O}_{2} / \mathrm{C}_{18} \mathrm{H}_{18} \mathrm{~F}_{2} \mathrm{O}_{2}$ : [M]+Na=325.1011/327.1167.

Found: $325.1016 / 327.1171$.<smiles>CCOC(=O)C(F)(F)F</smiles>

(E)-ethyl 5-(diethylamino)-2,2-difluoro-5-oxo-4-phenylpent-3-enoate

${ }^{1}$ H NMR (400 MHz, CDCl 3 ) $\delta$ ppm 7.30 (s, 5H), 6.72 (t, $\left.J=10.8 \mathrm{~Hz}, 1 \mathrm{H}\right), 3.97$ (q, $J=7.2$ $\mathrm{Hz}, 2 \mathrm{H}), 1.19$ (t, $J=7.2 \mathrm{~Hz}, 3 \mathrm{H})$.

${ }^{13}$ C NMR (100 MHz, CDCl $) \delta$ ppm $162.5(\mathrm{t}, J=33.1 \mathrm{~Hz}), 140.7,133.0(\mathrm{t}, J=28.2 \mathrm{~Hz})$, $129.4,128.0,127.8,110.8(\mathrm{t}, J=248.7 \mathrm{~Hz}), 108.7(\mathrm{t}, J=10.0 \mathrm{~Hz}), 63.1,13.7$.

${ }^{19}$ F NMR (376 MHz, CDCl 3$) \delta$ ppm $-93.9(\mathrm{~d}, J=7.5 \mathrm{~Hz})$.

IR (neat, $\mathrm{cm}^{-1}$ ): 2924, 1769, 1656, 1451, 1261, 1194, 1079, 1023, 757, 695.

HRMS (ESI) Calcd. for $\mathrm{C}_{17} \mathrm{H}_{21} \mathrm{~F}_{2} \mathrm{NO}_{3}:[\mathrm{M}]+\mathrm{Na}=374.9664$. Found: 374.9670 .

\section{Crystallographic data of 4au.}<smiles>O=C(CC=C(C(=O)NC1CCCCC1)c1ccccc1)NC1CCCCC1</smiles> 


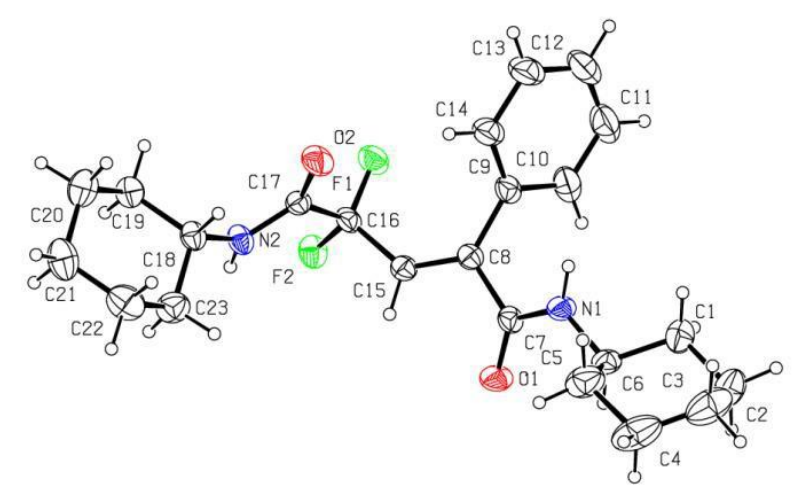

\section{Datablock:}

\begin{tabular}{lll}
\hline Bond precision: & $\mathrm{C}-\mathrm{C}=0.0050 \mathrm{~A}$ & Wavelength=0.71073 \\
Ce11: $\quad \begin{array}{lll}\mathrm{a}=13.5393(8) & \mathrm{b}=9.9243(4) & \mathrm{c}=17.0947(10) \\
\text { alpha=90 } & \text { beta=103.566(6) } & \text { gamma }=90\end{array}$ \\
Temperature: $295 \mathrm{~K}$ & &
\end{tabular}

$\begin{array}{lll} & \text { Calculated } & \text { Reported } \\ \text { Volume } & 2232.9(2) & 2232.9(2) \\ \text { Space group } & \text { P 21/n } & \text { P } 121 / \mathrm{n} 1 \\ \text { Hal1 group } & -\mathrm{P} \mathrm{2yn} & -\mathrm{P} 2 \mathrm{yn} \\ \text { Moiety formula } & \text { C23 H30 F2 N2 02 } & \text { C23 H30 F2 N2 02 } \\ \text { Sum formula } & \text { C23 H30 F2 N2 02 } & \text { C23 H30 F2 N2 } 02 \\ \text { Mr } & 404.49 & 404.49 \\ \text { Dx, g cm-3 } & 1.203 & 1.203 \\ \text { Z } & 4 & 4 \\ \text { Mu (mm-1) } & 0.088 & 0.088 \\ \text { F000 } & 864.0 & 864.0 \\ \text { F000' } & 864.44 & \\ \text { h, k, 1max } & 16,12,21 & 16,12,21 \\ \text { Nref } & 4405 & 4399 \\ \text { Tmin, Tmax } & 0.983,0.988 & 0.583,1.000 \\ \text { Tmin' } & 0.983 & \end{array}$

Correction method $=$ \# Reported T Limits: Tmin=0.583 Tmax $=1.000$ AbsCorr $=$ MULTI-SCAN 
6. ${ }^{1} \mathrm{H}$ NMR、 ${ }^{13} \mathrm{C}$ NMR $、{ }^{19}$ F NMR Spectra for Substrates 3aa-3va, 4ab-4ag, 4aj-4au, 5,6 and 7

Data completeness $=0.999$

$\mathrm{R}($ reflections $)=0.0632(2541)$

$S=1.047 \quad$ Npar $=262$

$\mathrm{S}=1.047 \quad$ Npar $=262$
Theta $(\max )=26.020$

$\mathrm{wR} 2($ reflections $)=0.2101(4399)$ 
mºํ.

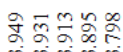

$\stackrel{\infty}{=} \stackrel{m}{=}$

$\stackrel{8}{8}$

$\mathrm{EtoOCF}_{2} \mathrm{C}$

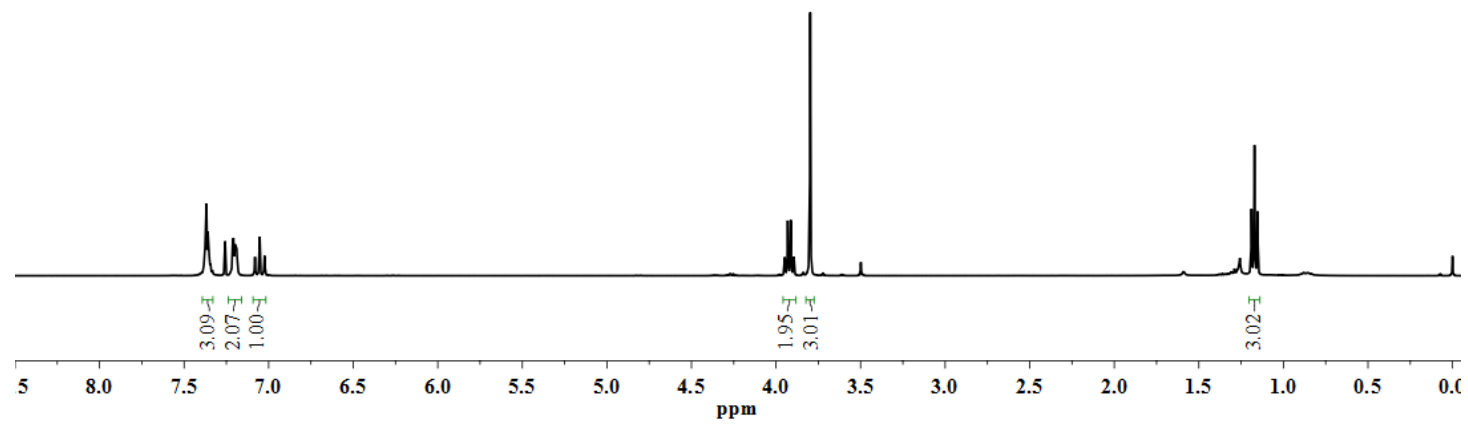

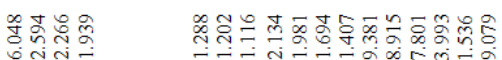

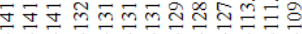

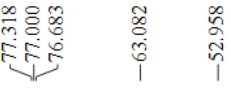

$\stackrel{5}{n}$

EtoocF 2 OMa

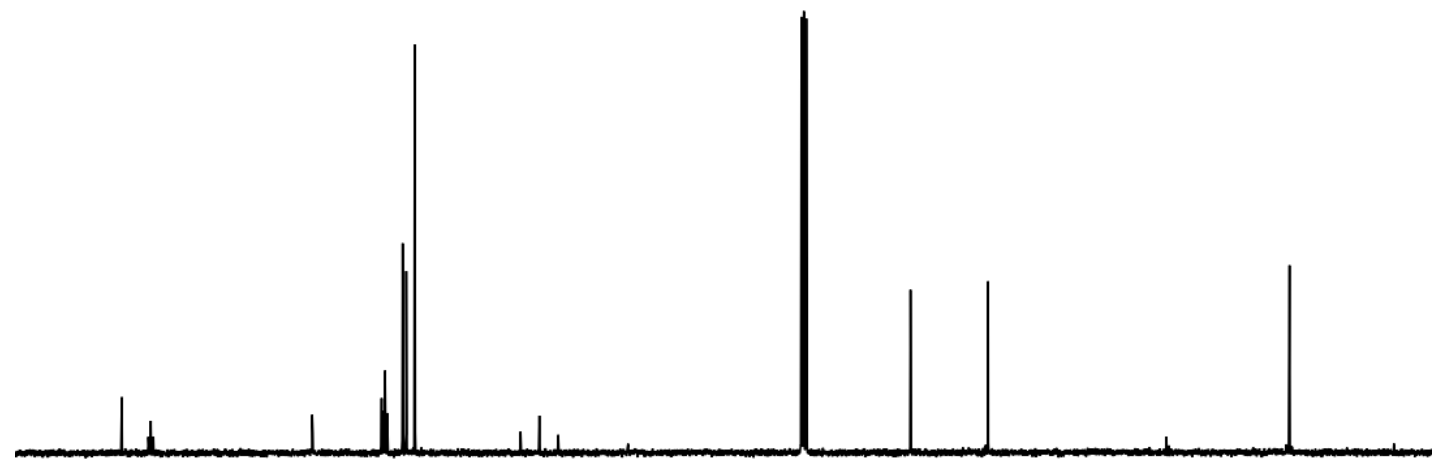

160

140

120

100

80

60

40

20

0 


$$
\text { EtoocF } 2 \text { 3aa }
$$

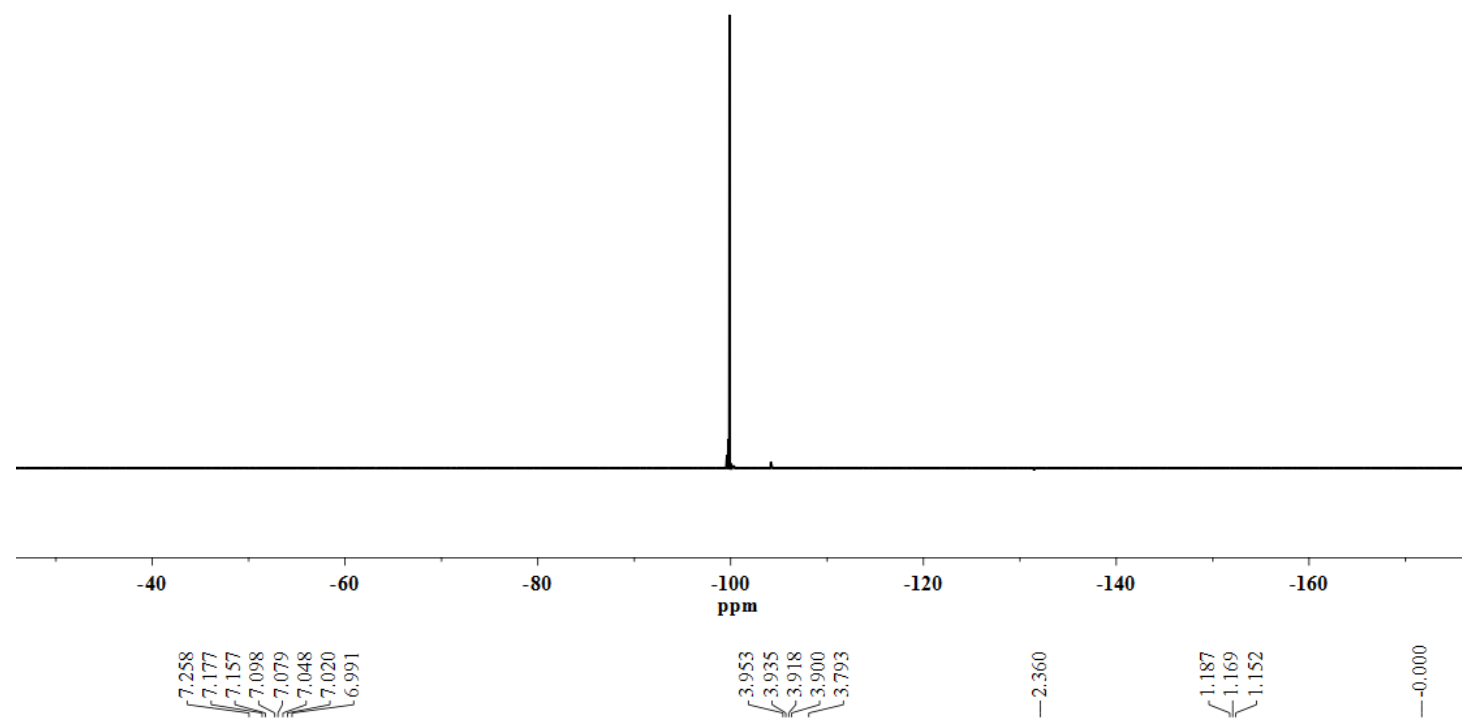

$$
\text { EtooCF } 2 \text { Come }
$$

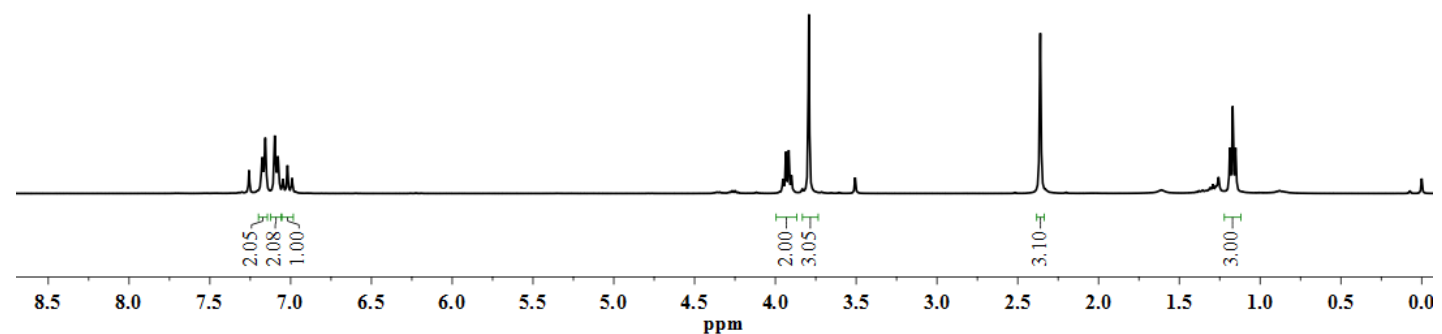




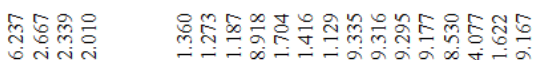

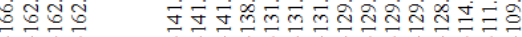

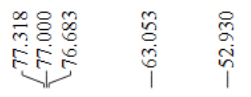

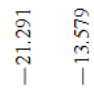

EtooCF 2 OMba
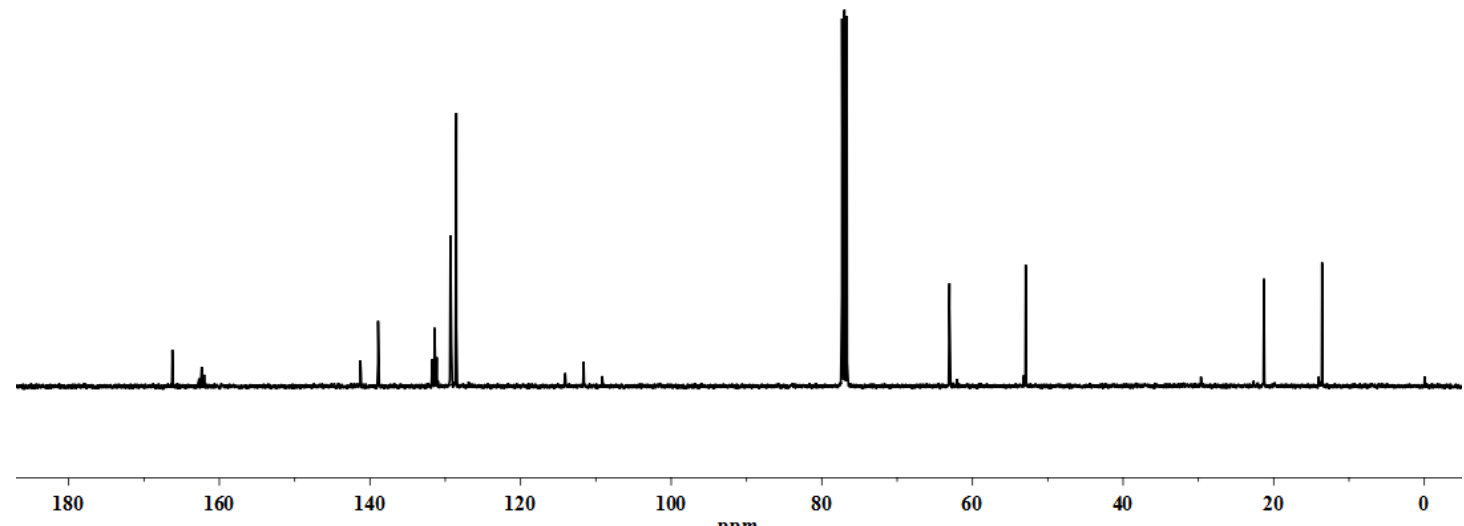

120

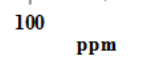

$80 \quad 60$

品

$\mathrm{EtOOCF}_{2} \mathrm{C}$

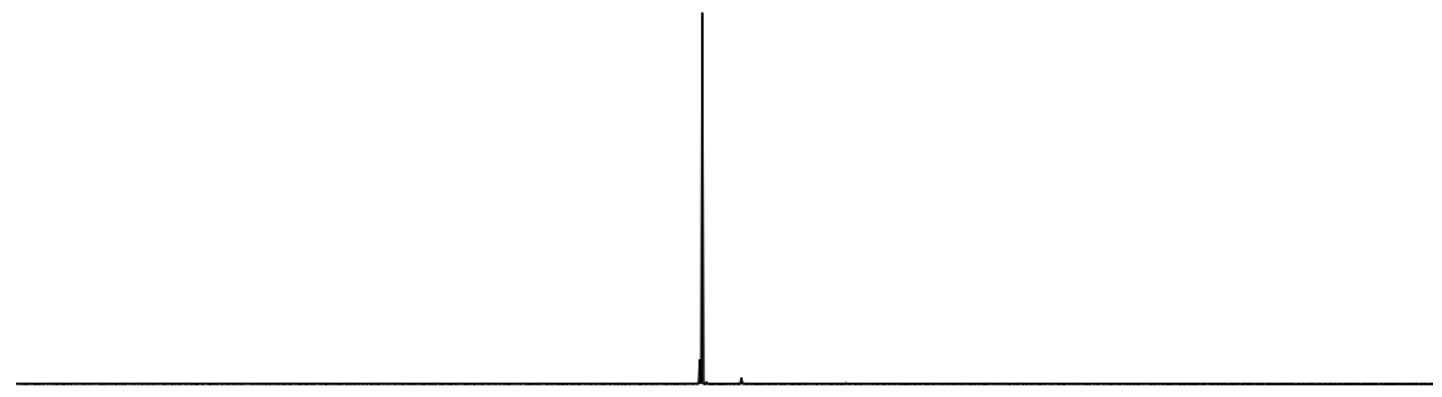

$-20$

$-40$

$-60$

$-80$

$\operatorname{ppm}^{-100}$

$-120$

$-140$

$-160$ 
m

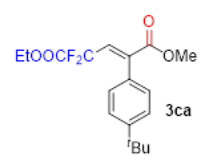

lulw

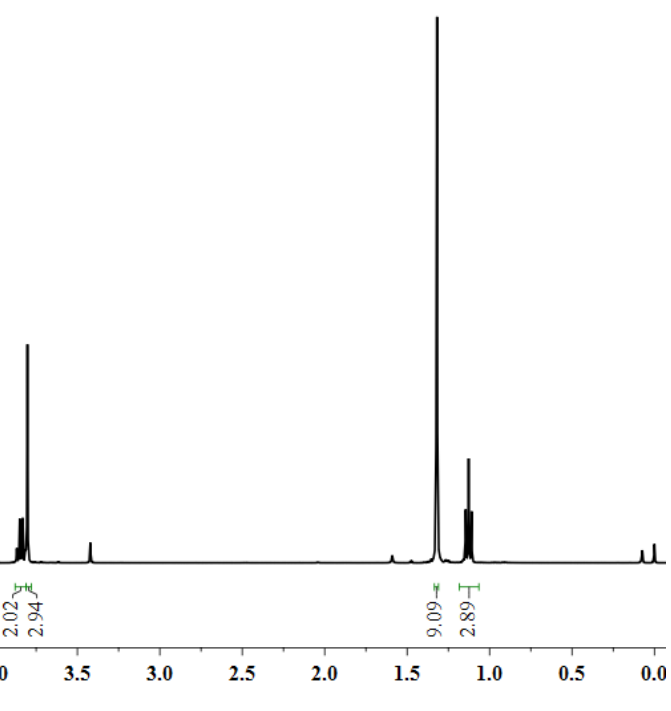

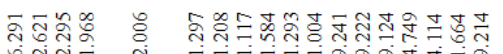

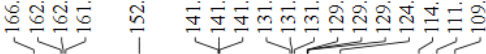

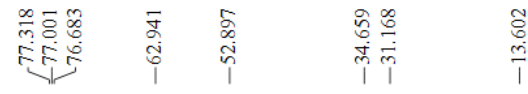

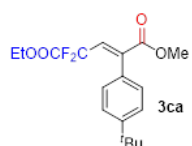

${ }^{\mathrm{t}} \mathrm{Bu}$

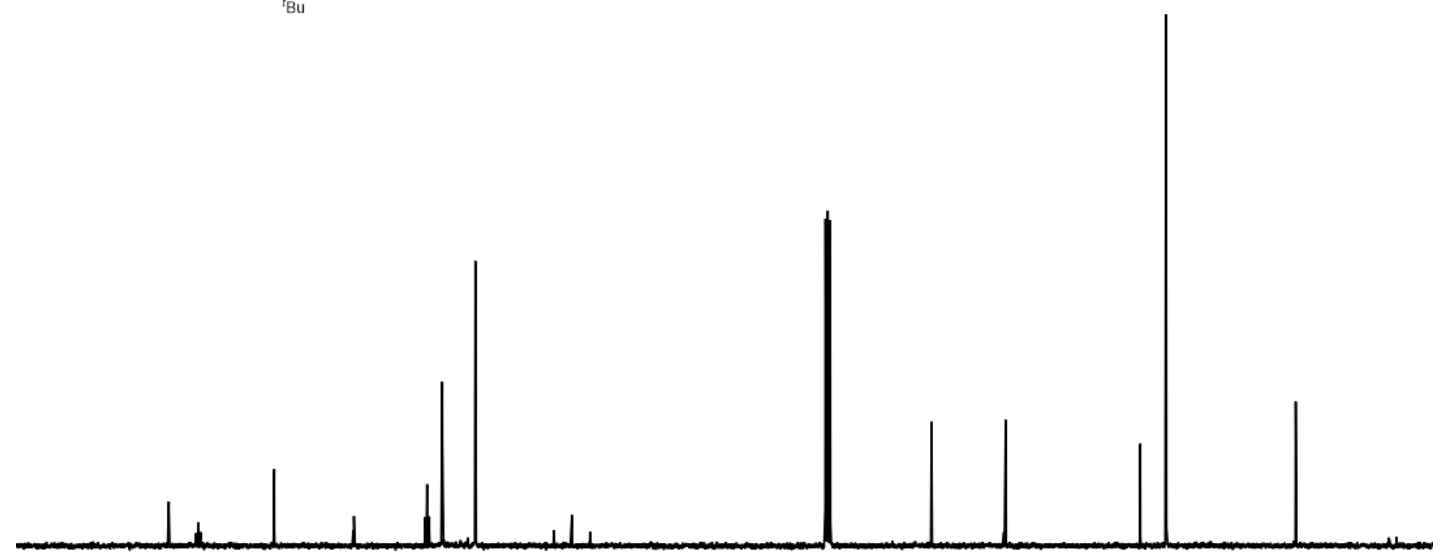

160

140

120

100

ppm

80

60

40

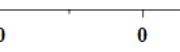


3ca

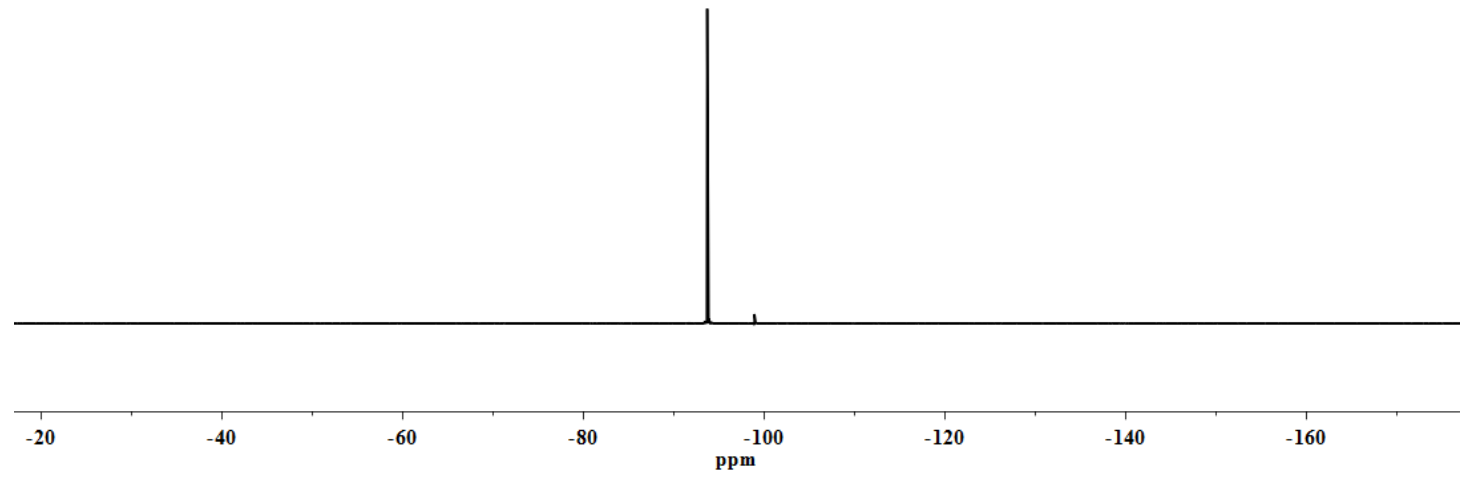

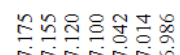

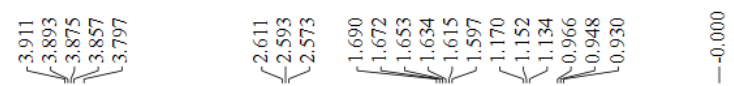

EtoocF $2 \overbrace{3 \mathrm{C}}^{\mathrm{OMme}}$

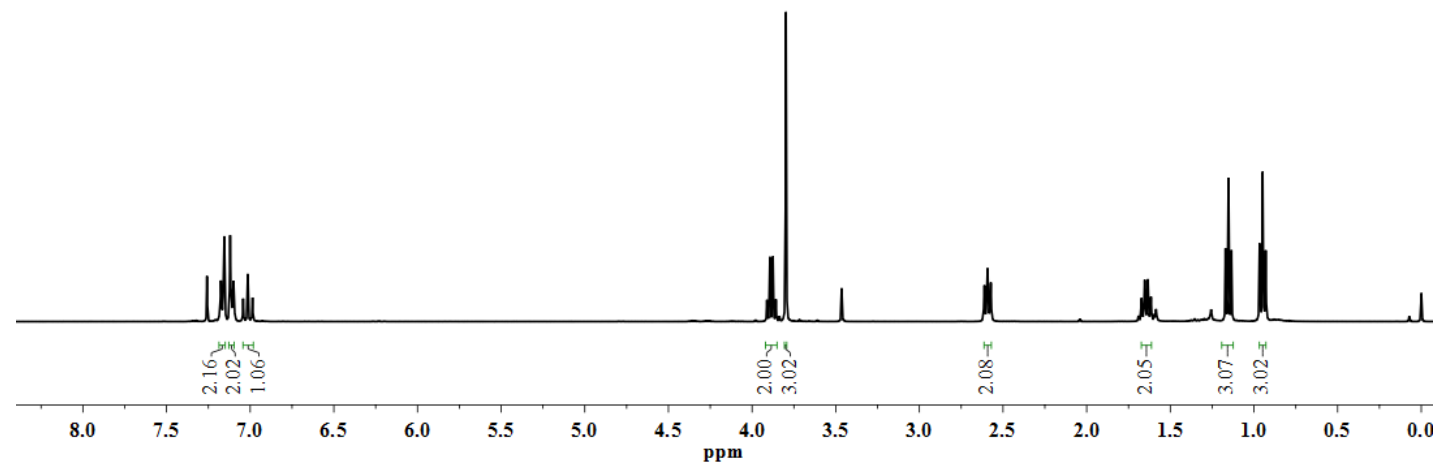




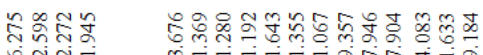

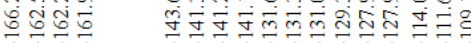

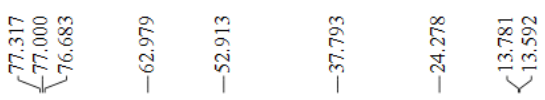

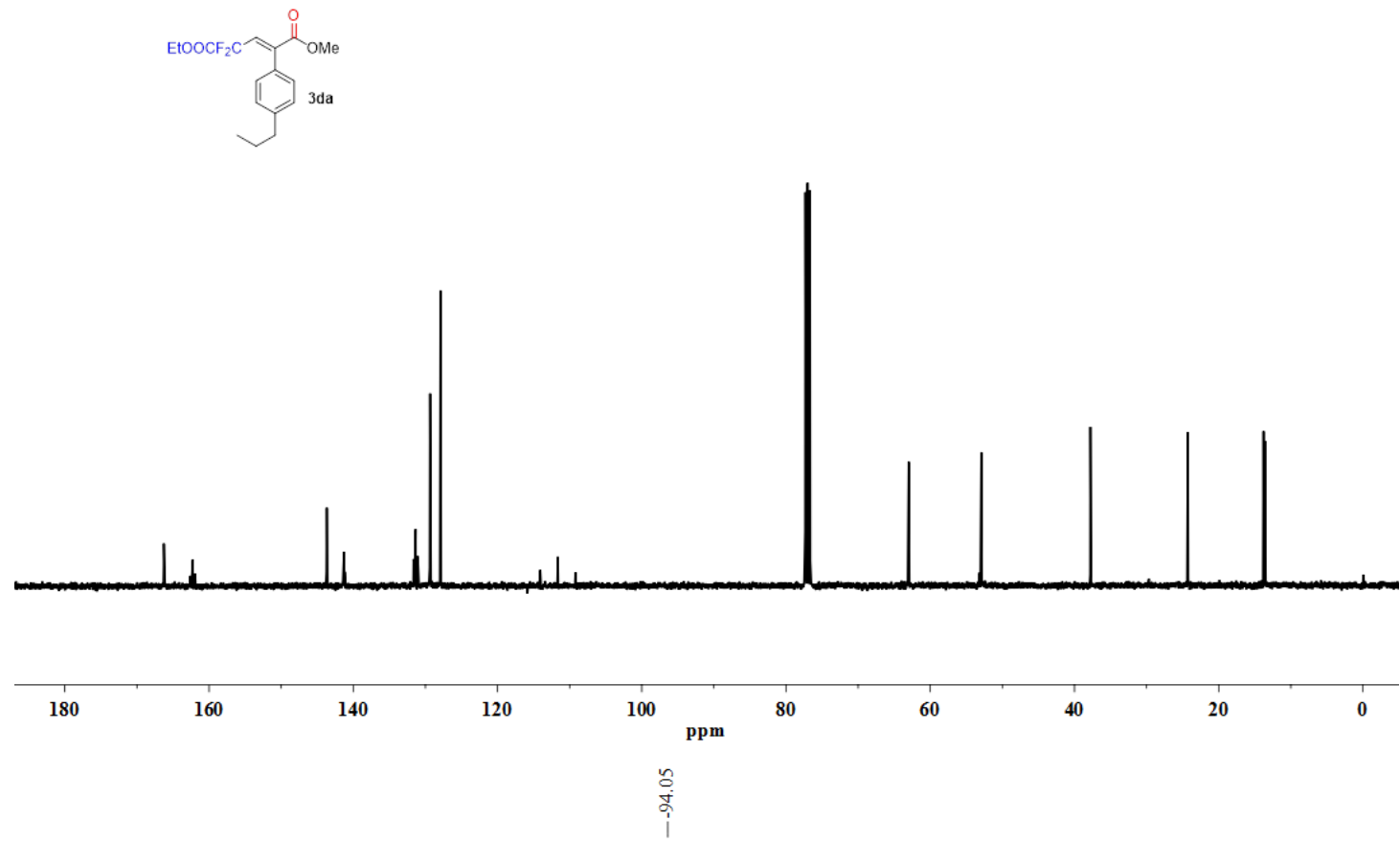

$\mathrm{EtOOCF}_{2} \mathrm{C} \overbrace{3 \mathrm{da}}^{\mathrm{O}}$

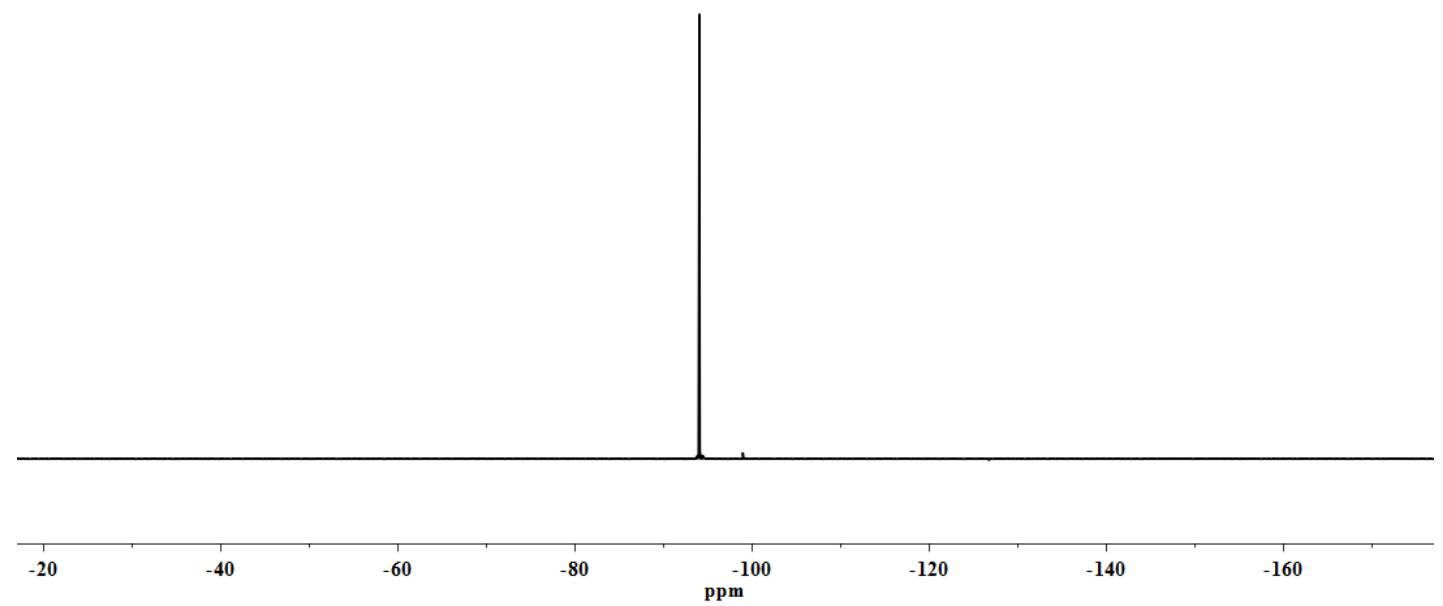




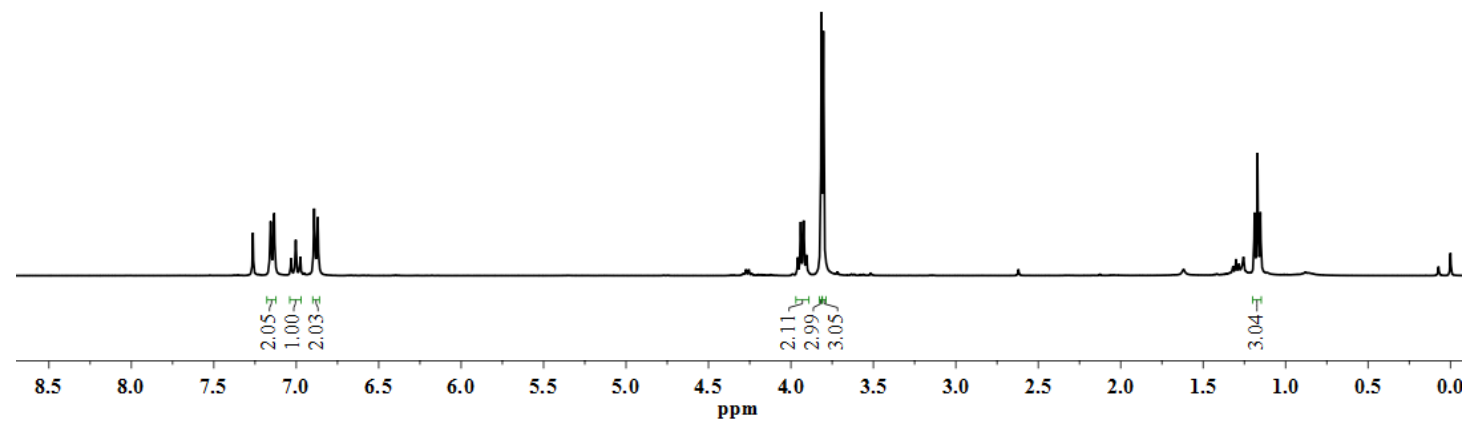

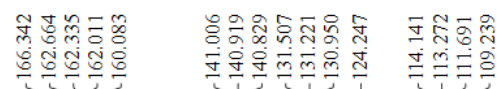

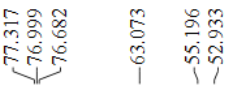

EtoOCF 2

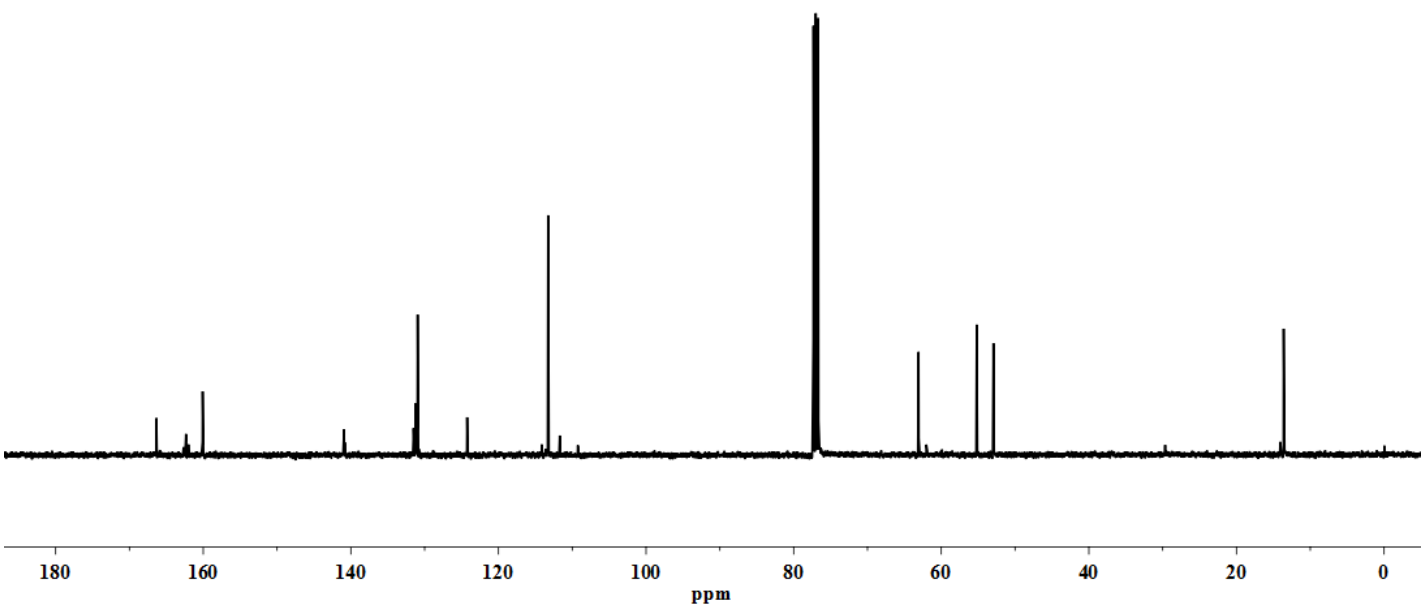


EtoocF 2 C OMea

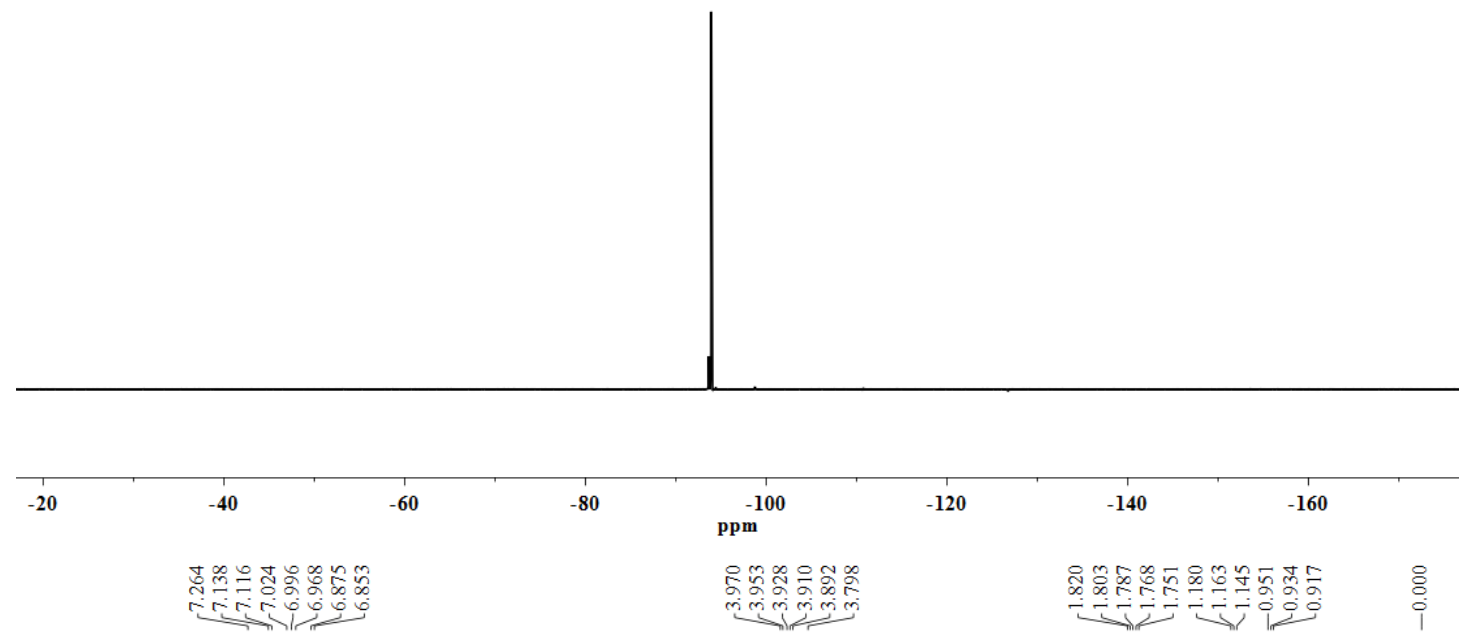

3ta

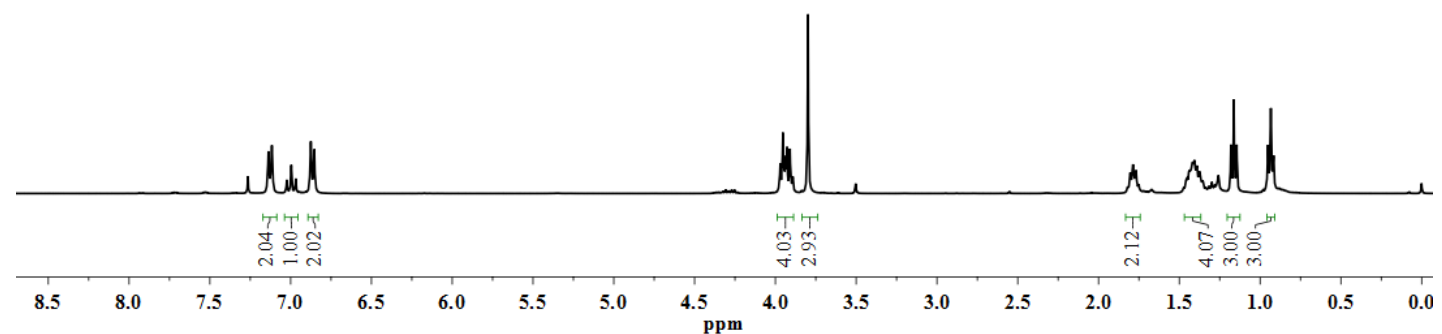




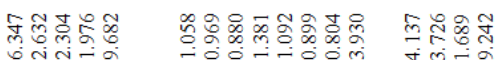

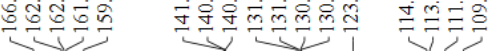

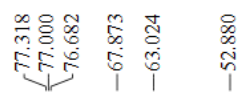

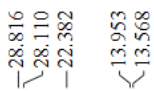
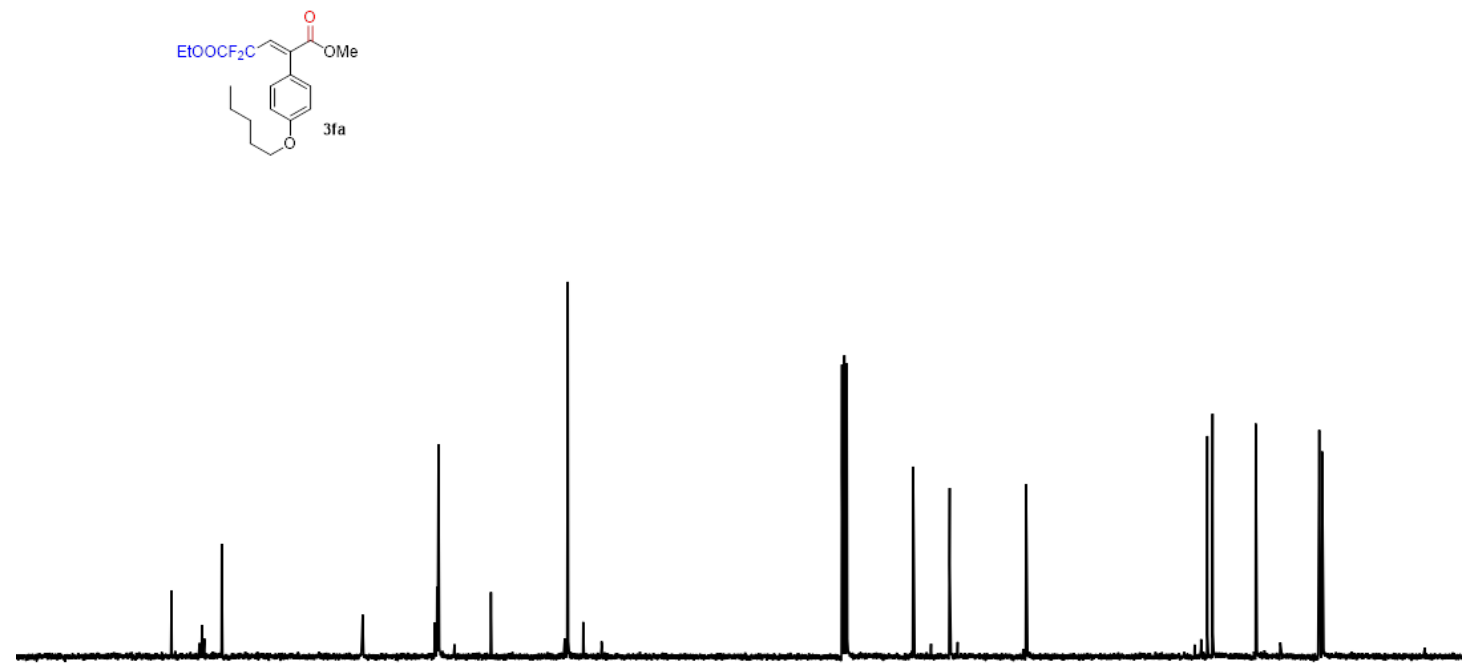

180
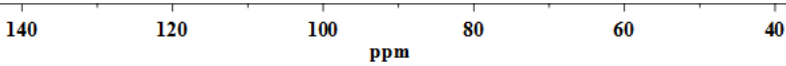

$\stackrel{\circ}{\grave{i}}$
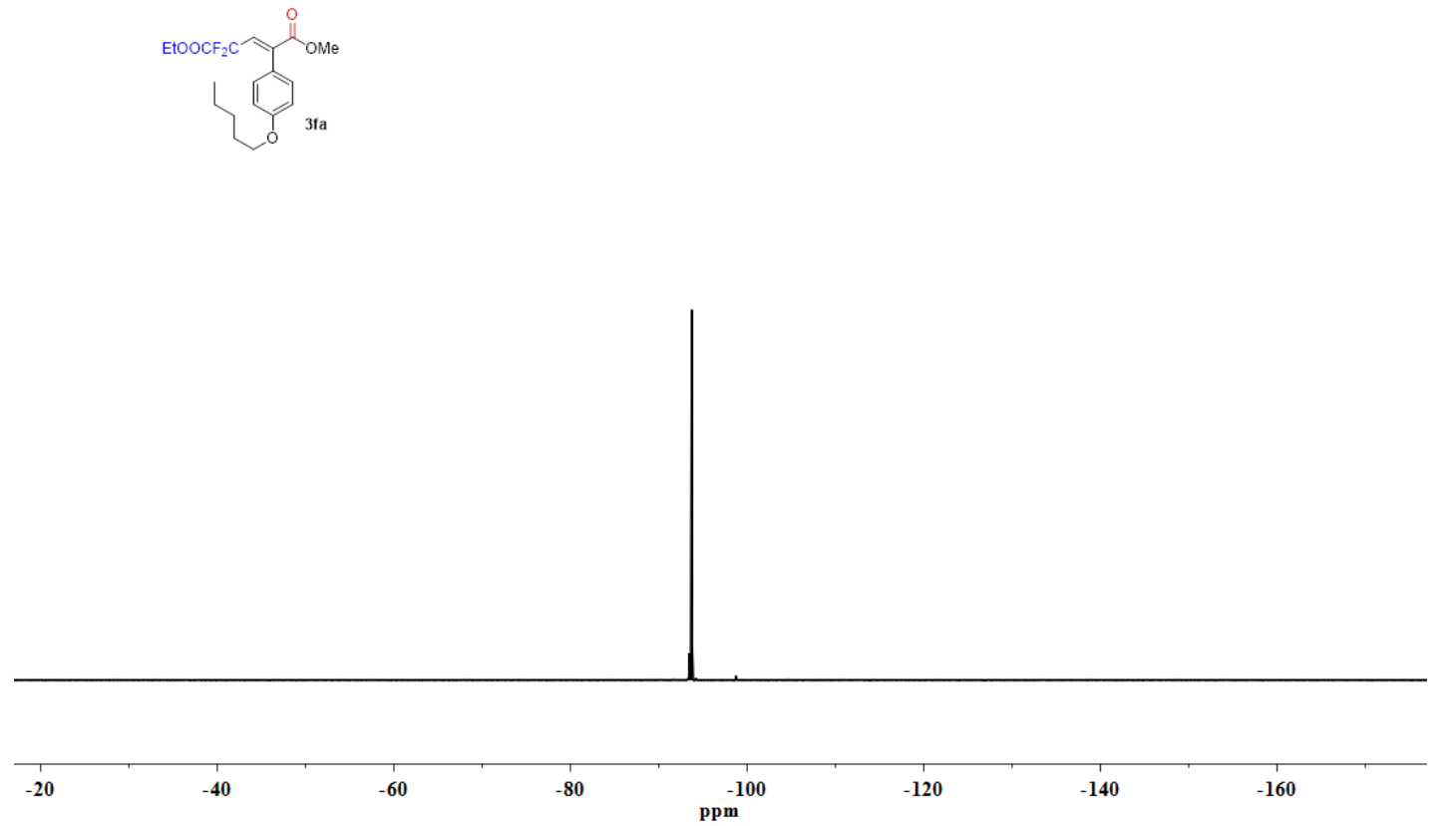


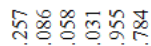

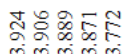

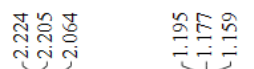

$\underset{\substack{0 \\ i}}{i}$
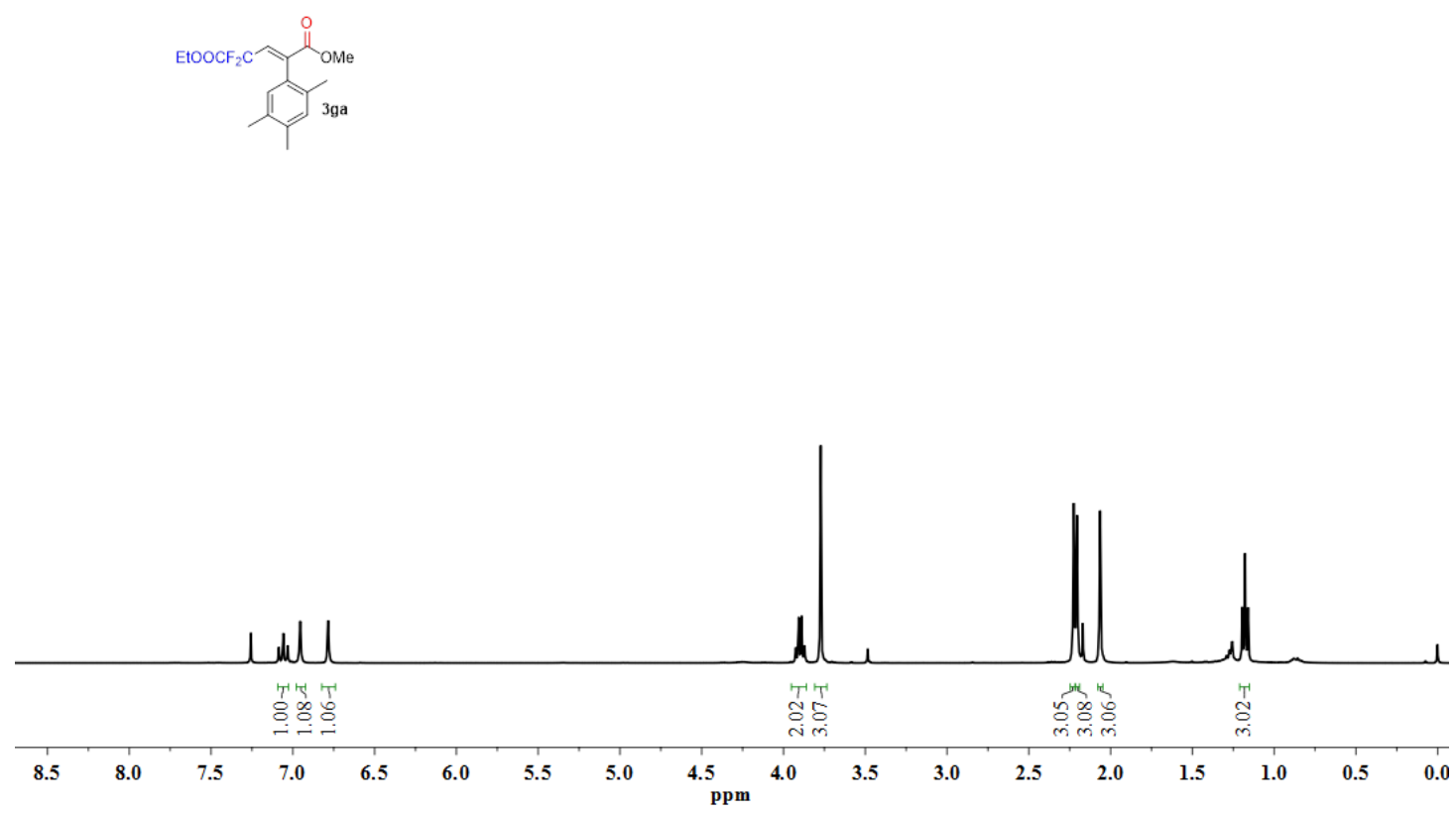

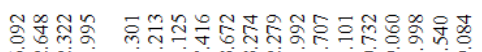

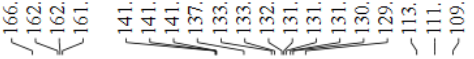

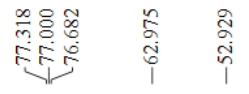

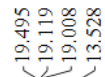
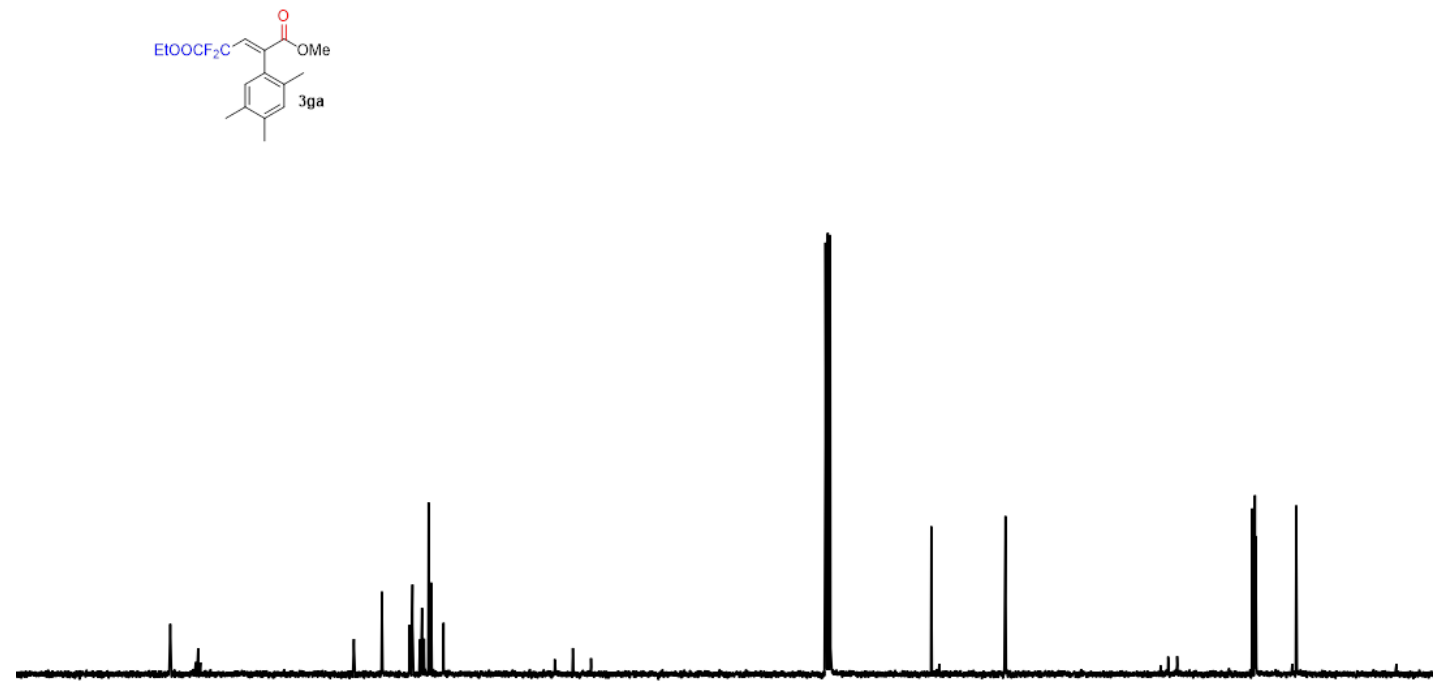

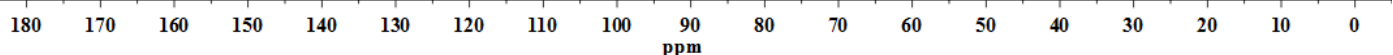




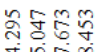

के

EtoocF $\mathrm{C}_{3}^{\mathrm{H}}$

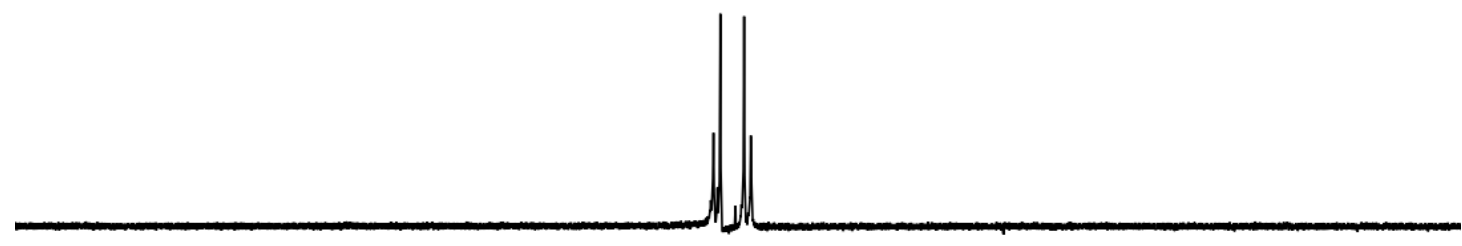

\begin{tabular}{llllllllllllllll}
\hline-20 & -30 & -40 & -50 & -60 & -70 & -80 & -90 & $\begin{array}{c}1 \\
\mathrm{pm}\end{array}$ & -110 & -120 & -130 & -140 & -150 & -160 & -170
\end{tabular}

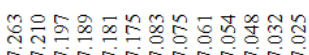

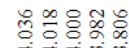

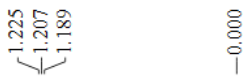

EtoocF 20 Come

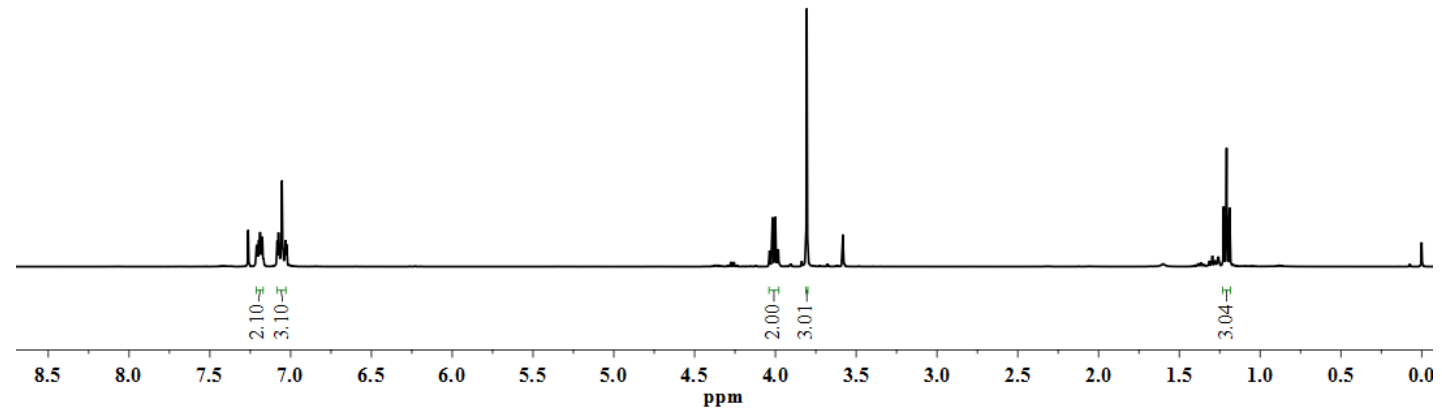




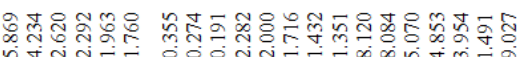

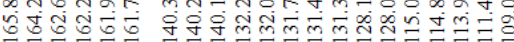

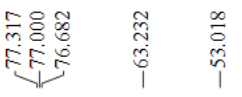

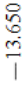
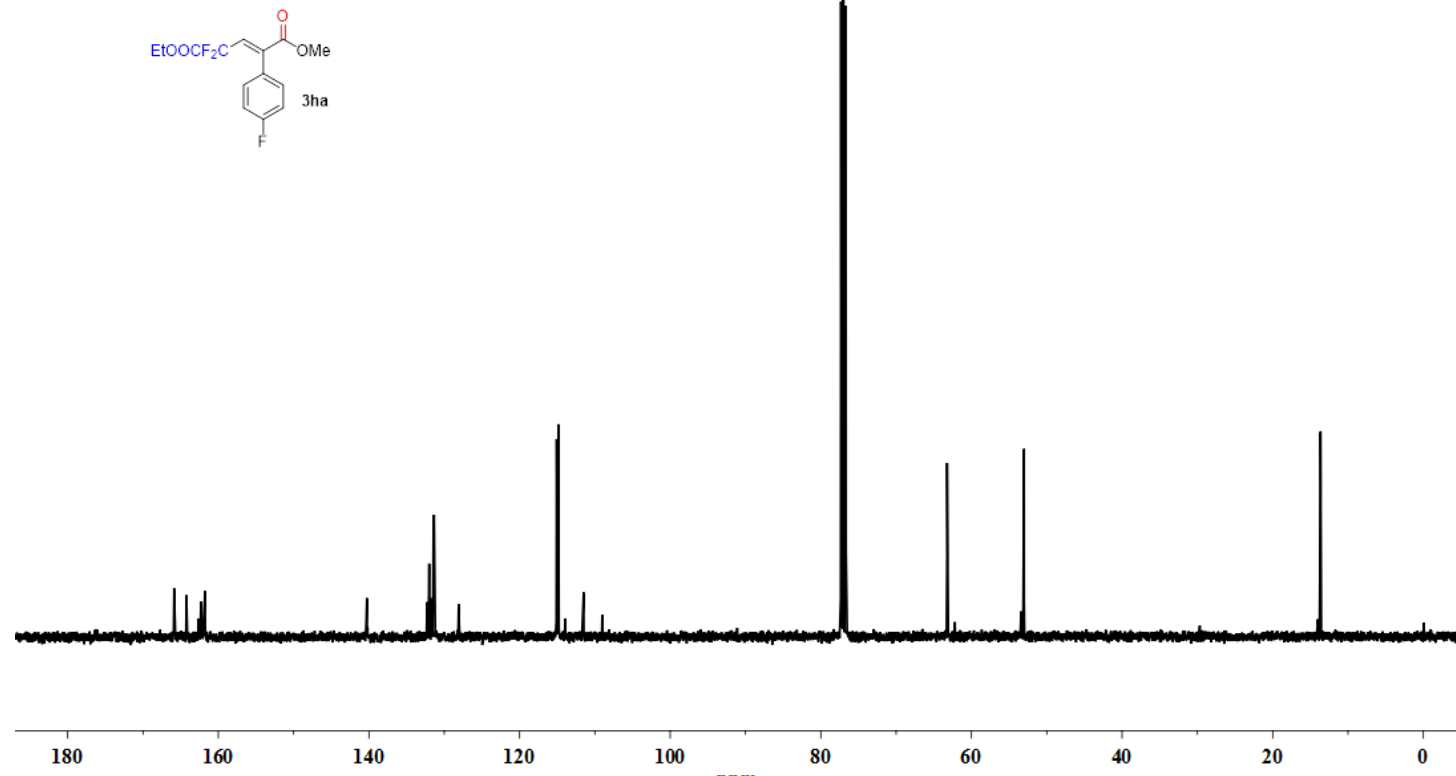

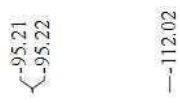

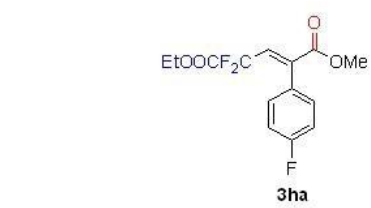

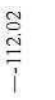

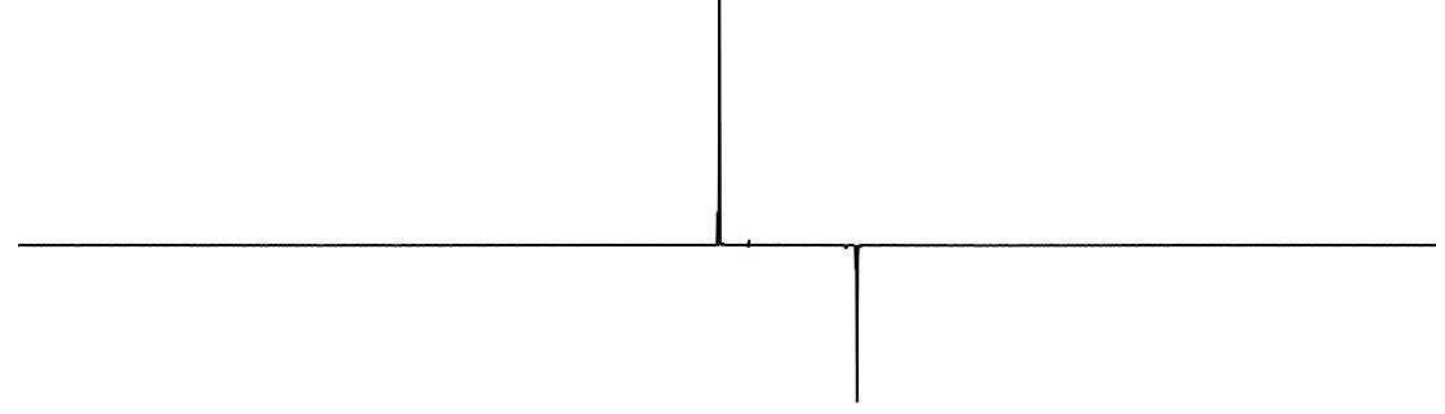

\begin{tabular}{|c|c|c|c|c|c|c|c|c|}
\hline-20 & -40 & -60 & -80 & $\mathrm{ppm}^{-100}$ & -120 & -140 & -160 & -180 \\
\hline
\end{tabular}




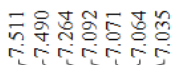

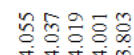

$\mathrm{EtOOCF}_{2} \mathrm{C}$

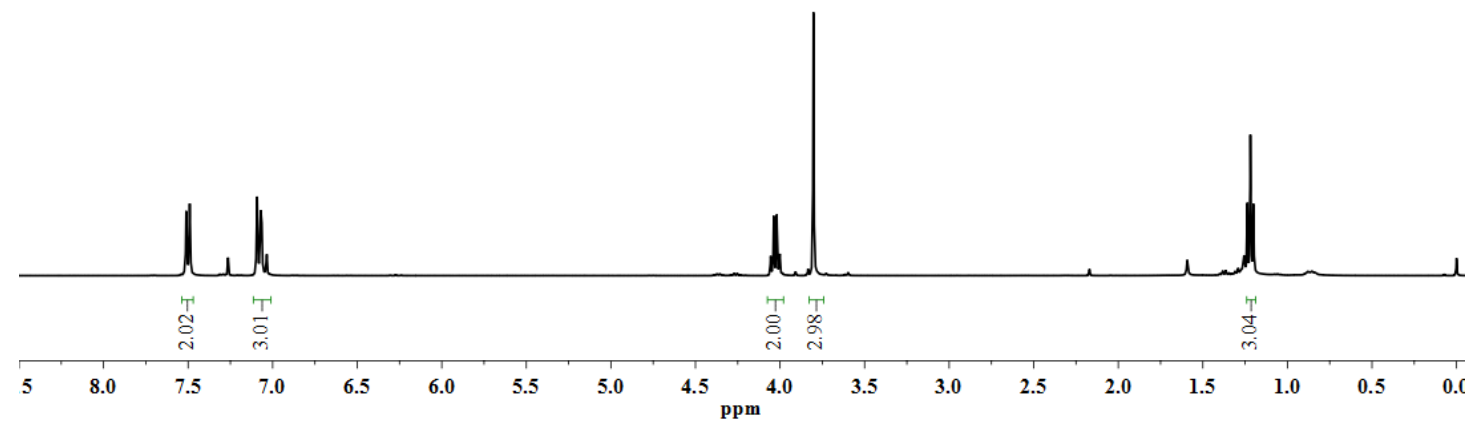

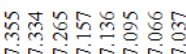

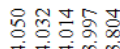

꼰

$\stackrel{8}{\circ}$

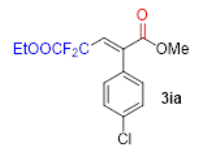

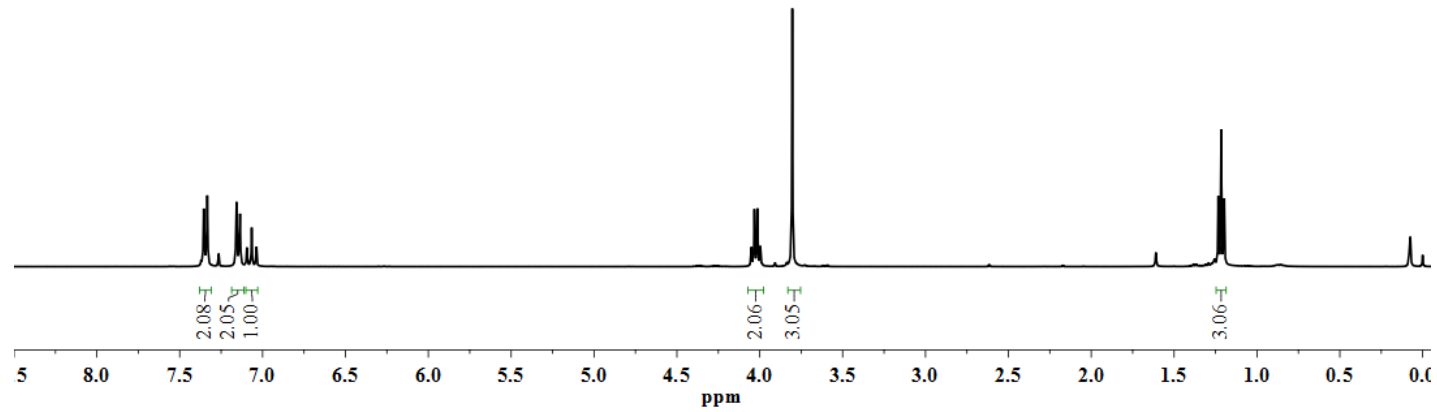




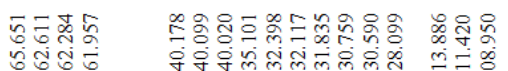

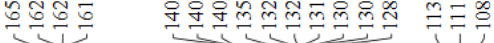

लेक्ष ते के के

$\underset{\substack{\text { gे } \\ \text { m }}}{\stackrel{0}{1}}$

EtoocF $20 \overbrace{\text { cl }}^{\text {Iome }}$

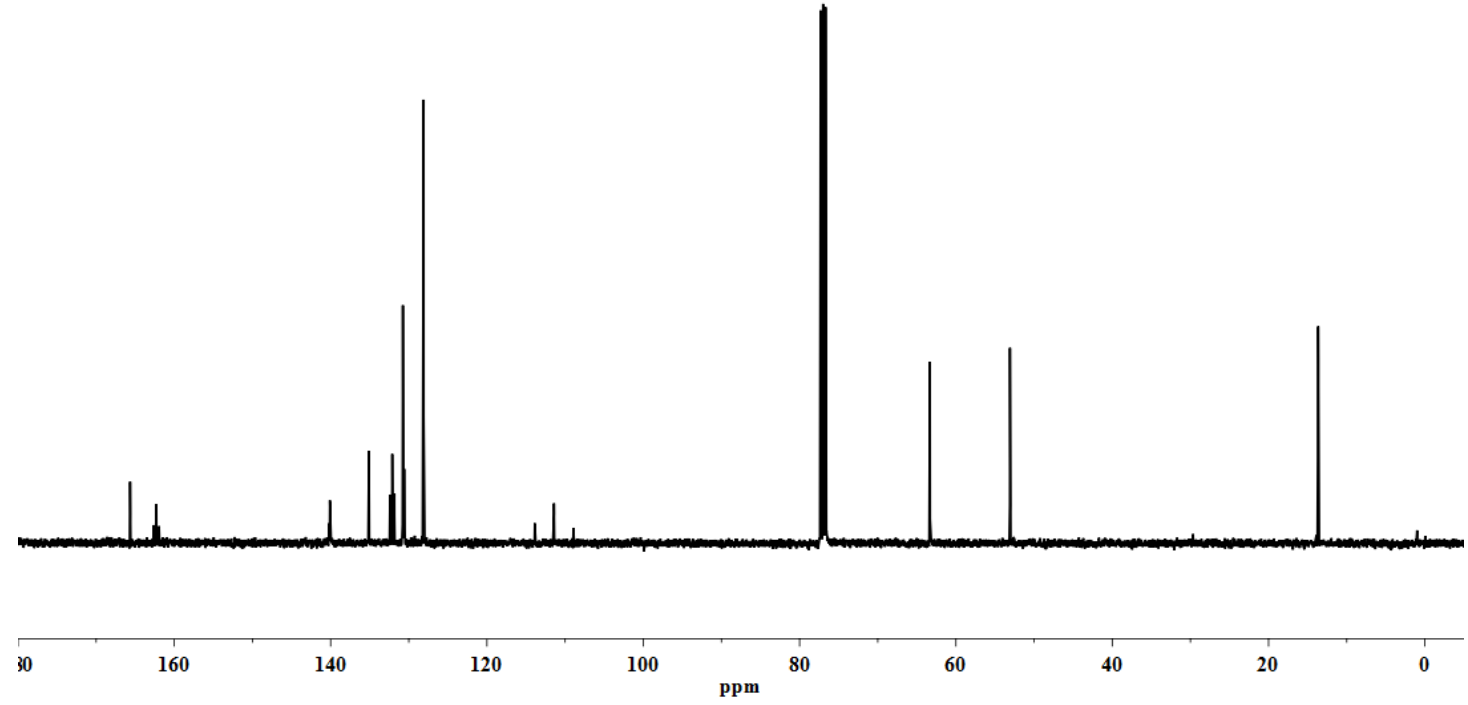

守

$\mathrm{EtOOCF}_{2} \mathrm{C}$

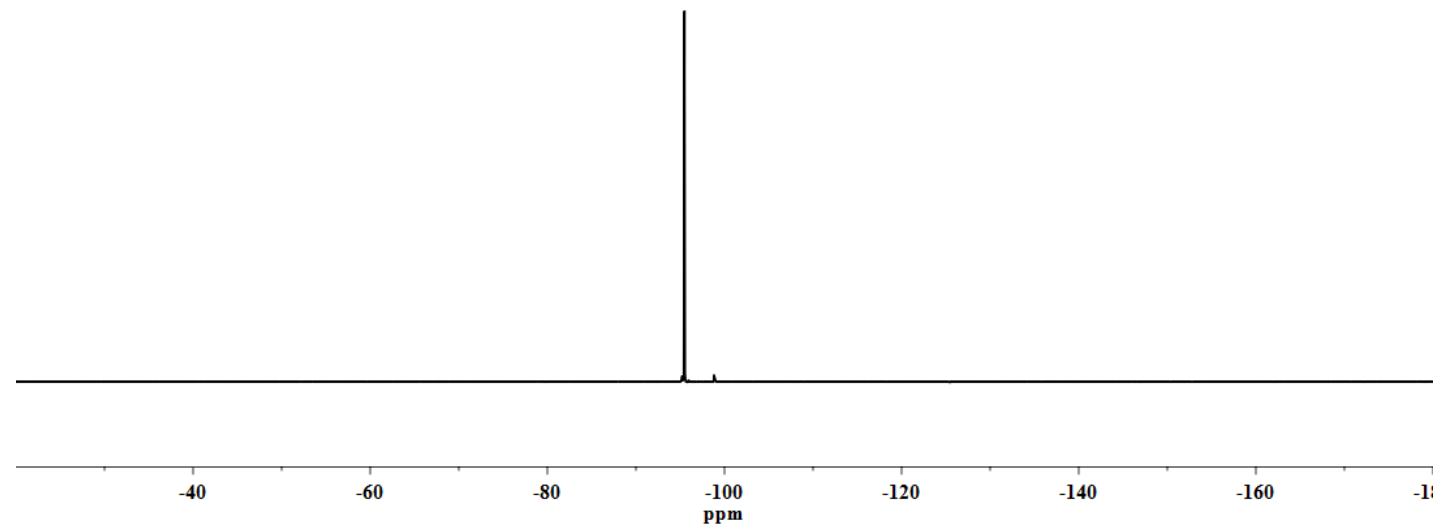




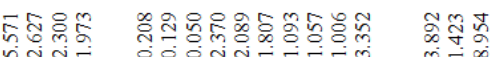

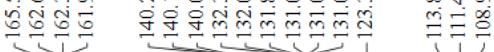

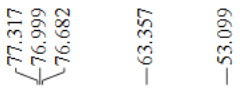

$\underset{\overparen{6}}{\stackrel{1}{7}}$
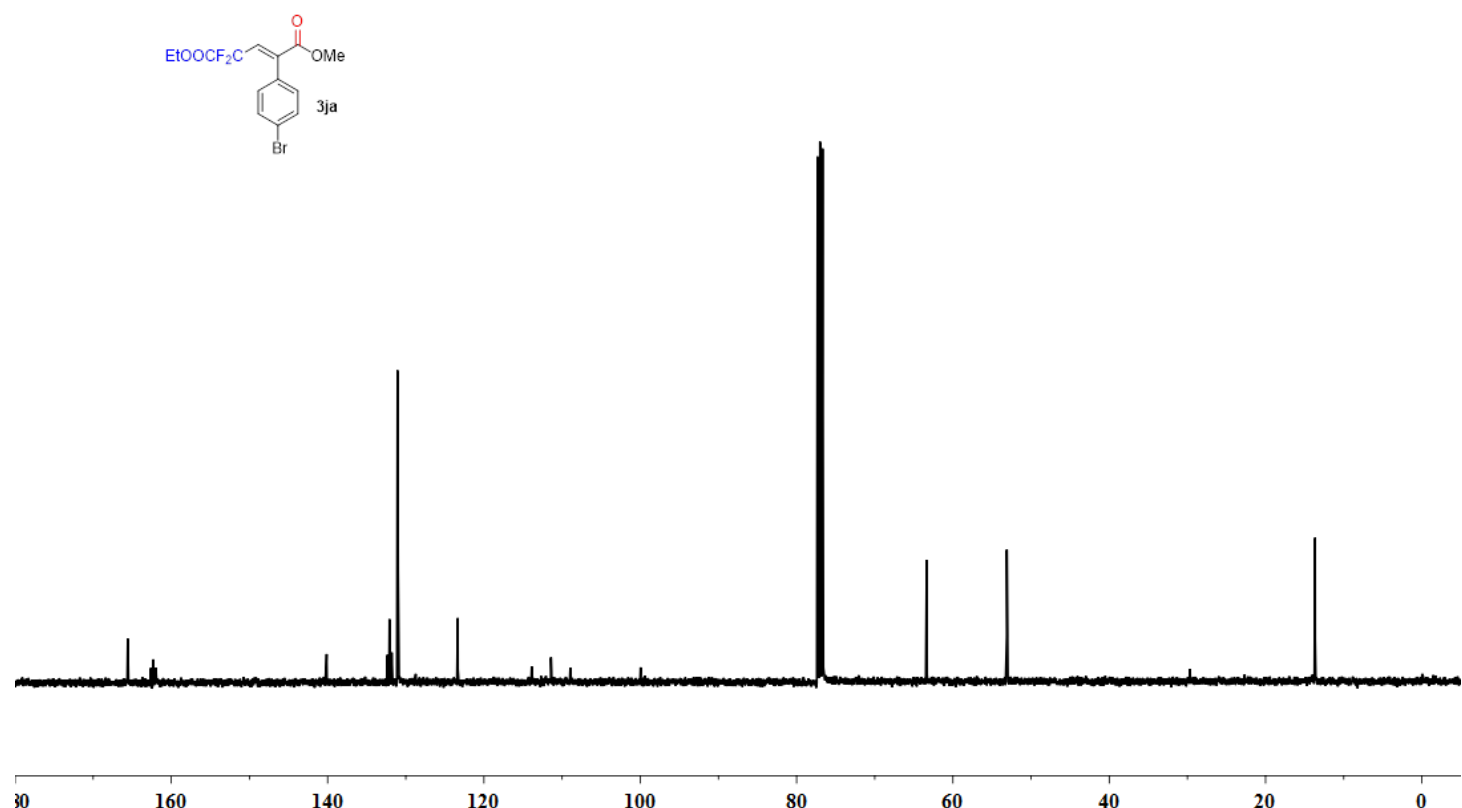

120

100

80

60

40

$20 \quad 0$

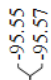

$\mathrm{EtOOCF}_{2} \mathrm{C}$

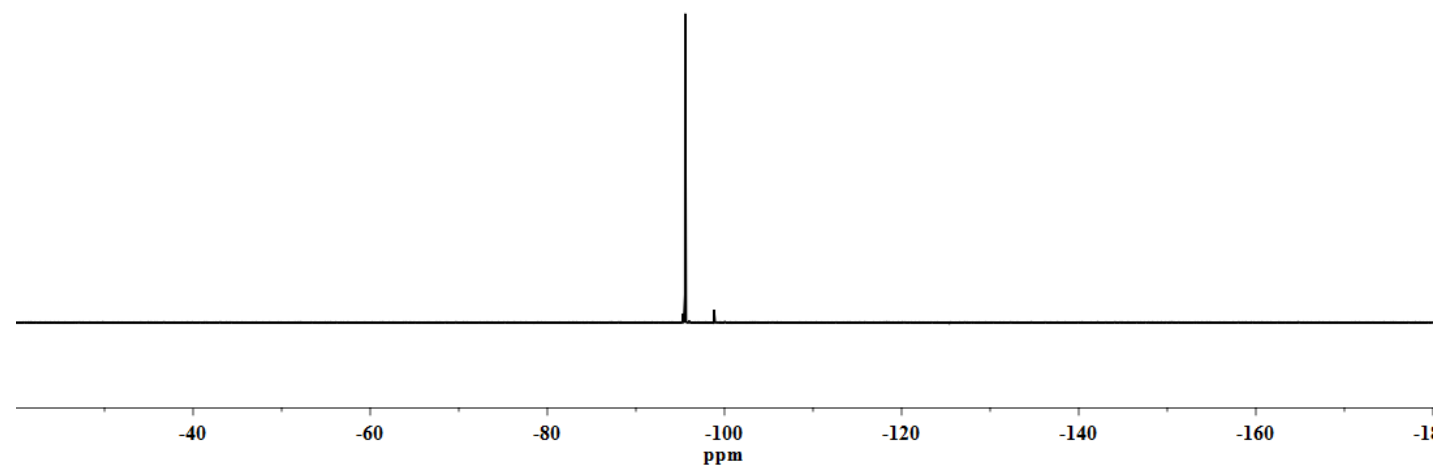




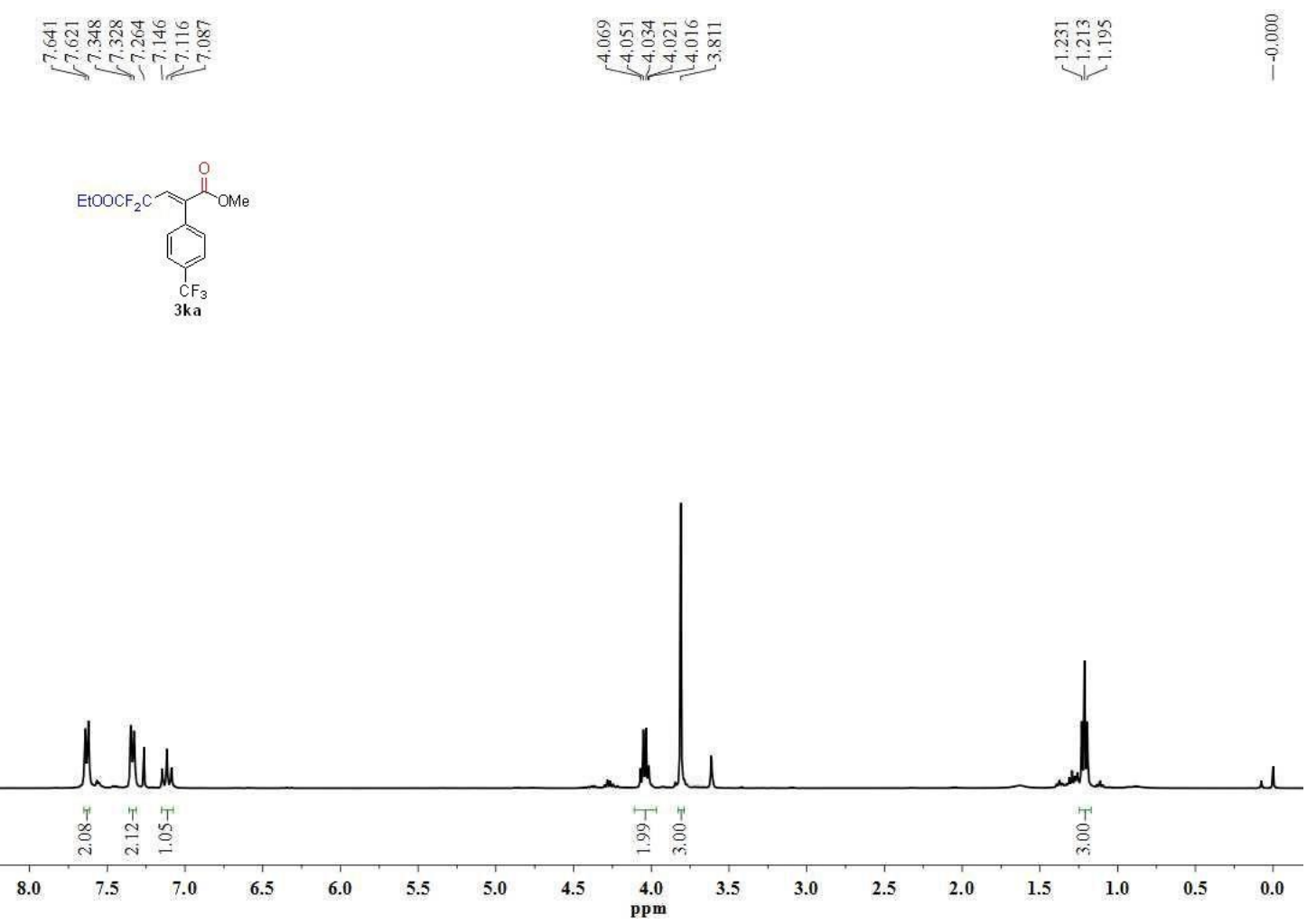

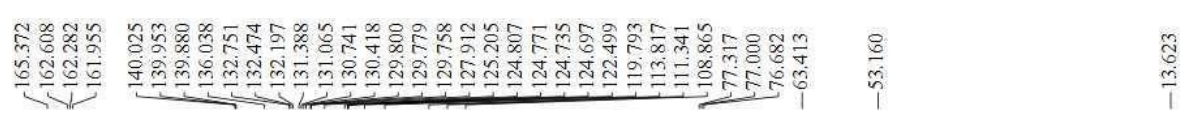
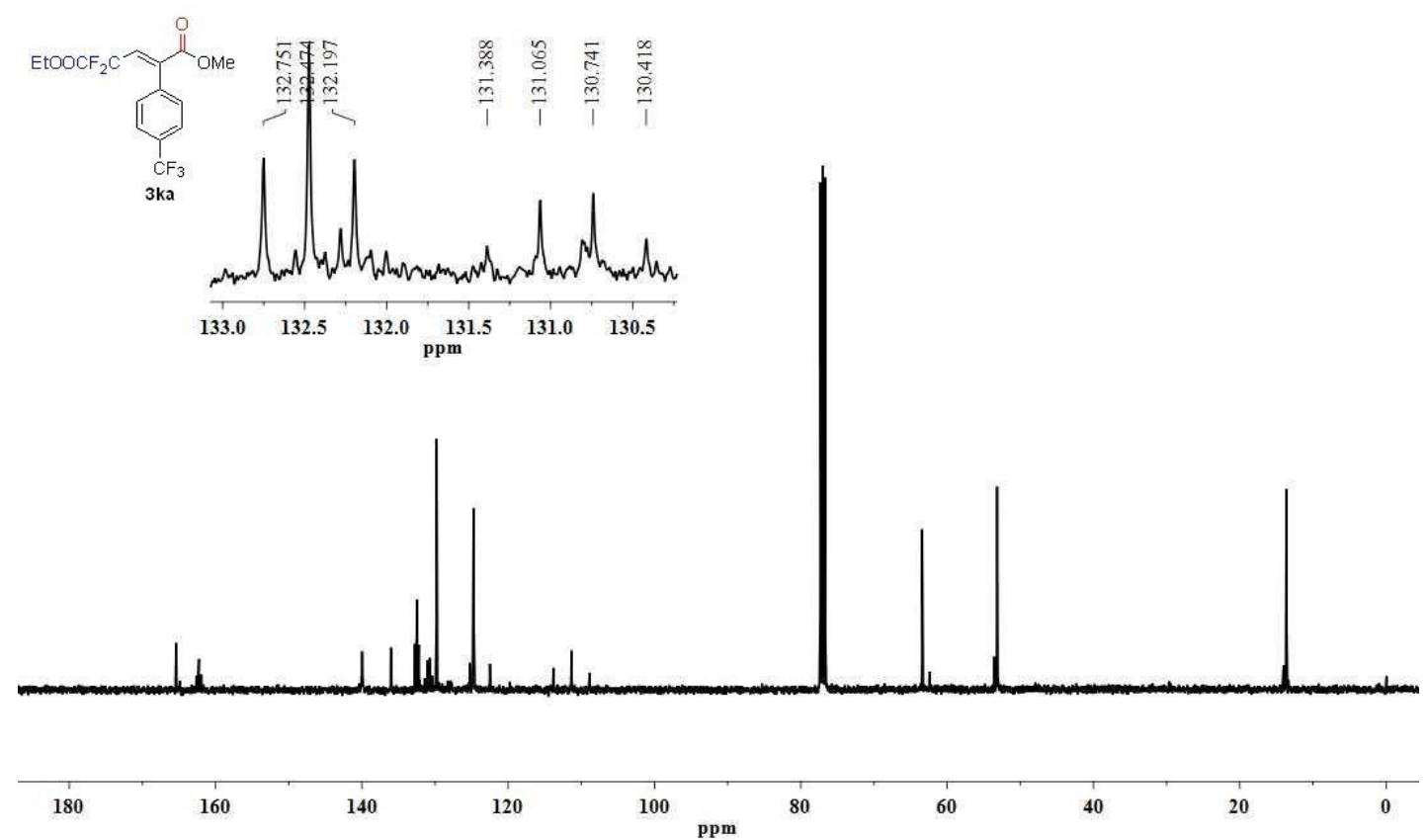


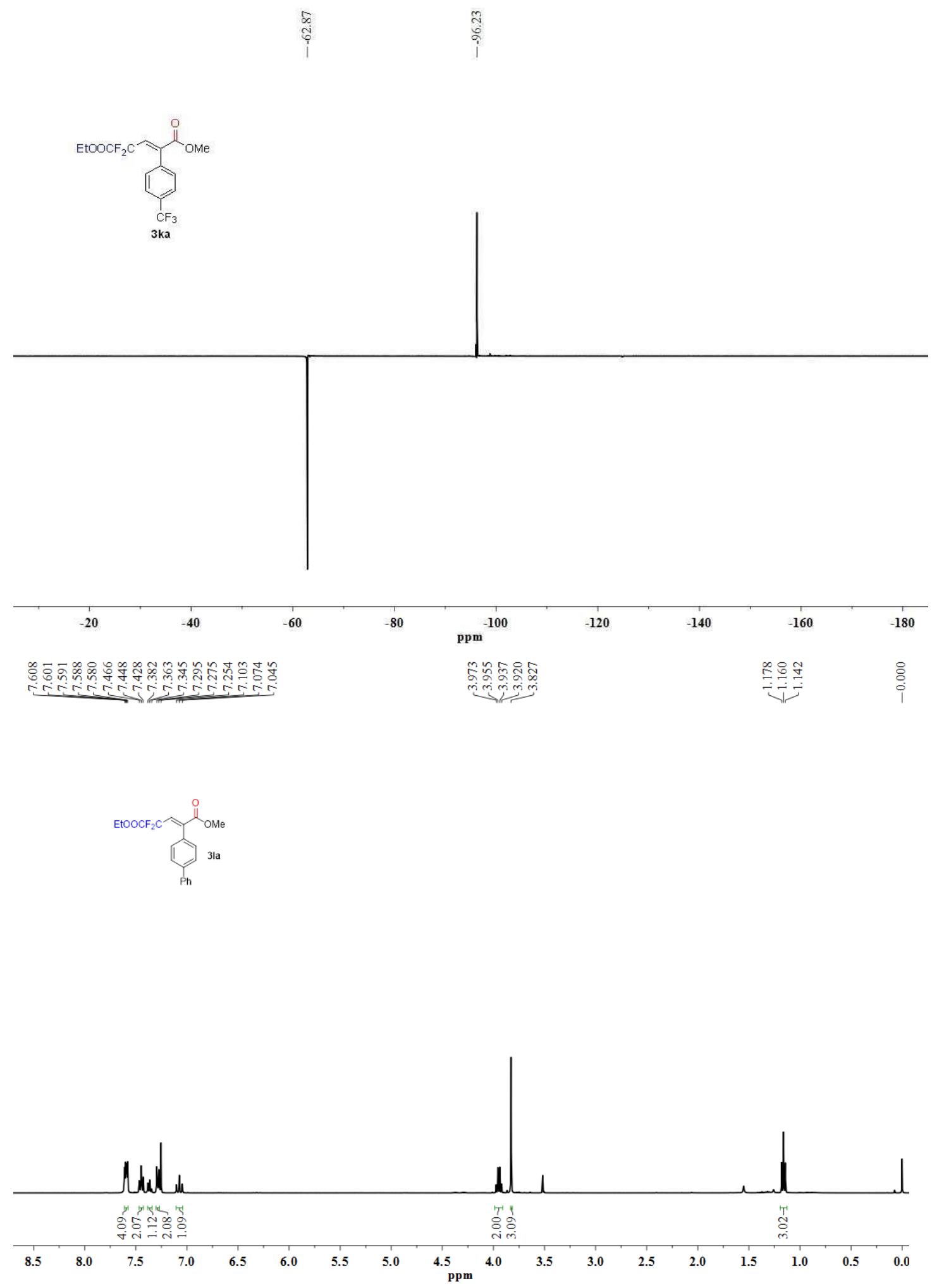




$$
\text { EtoocF } 2 \text { Cla }
$$

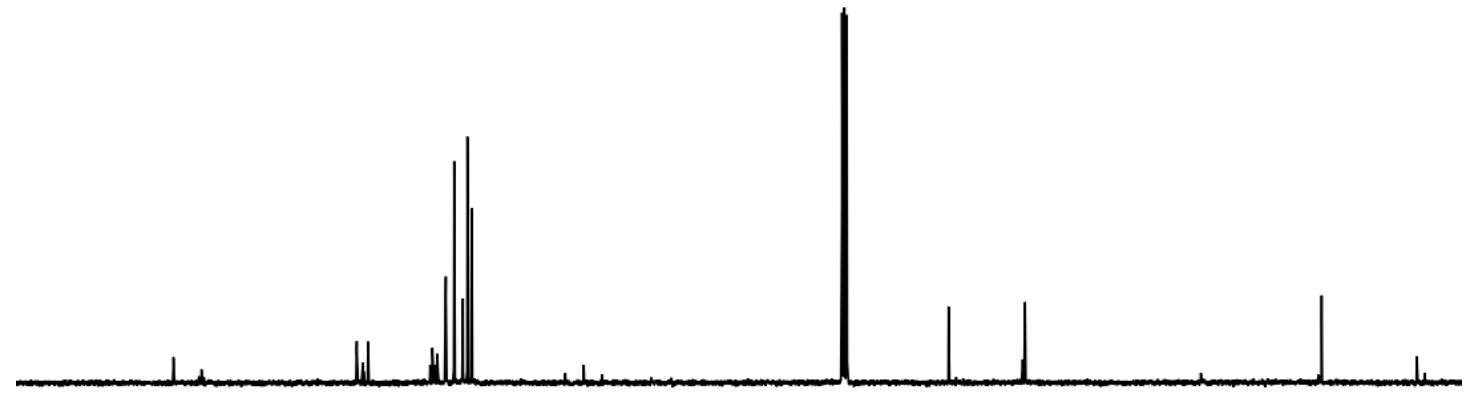

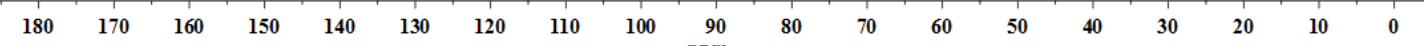

战管
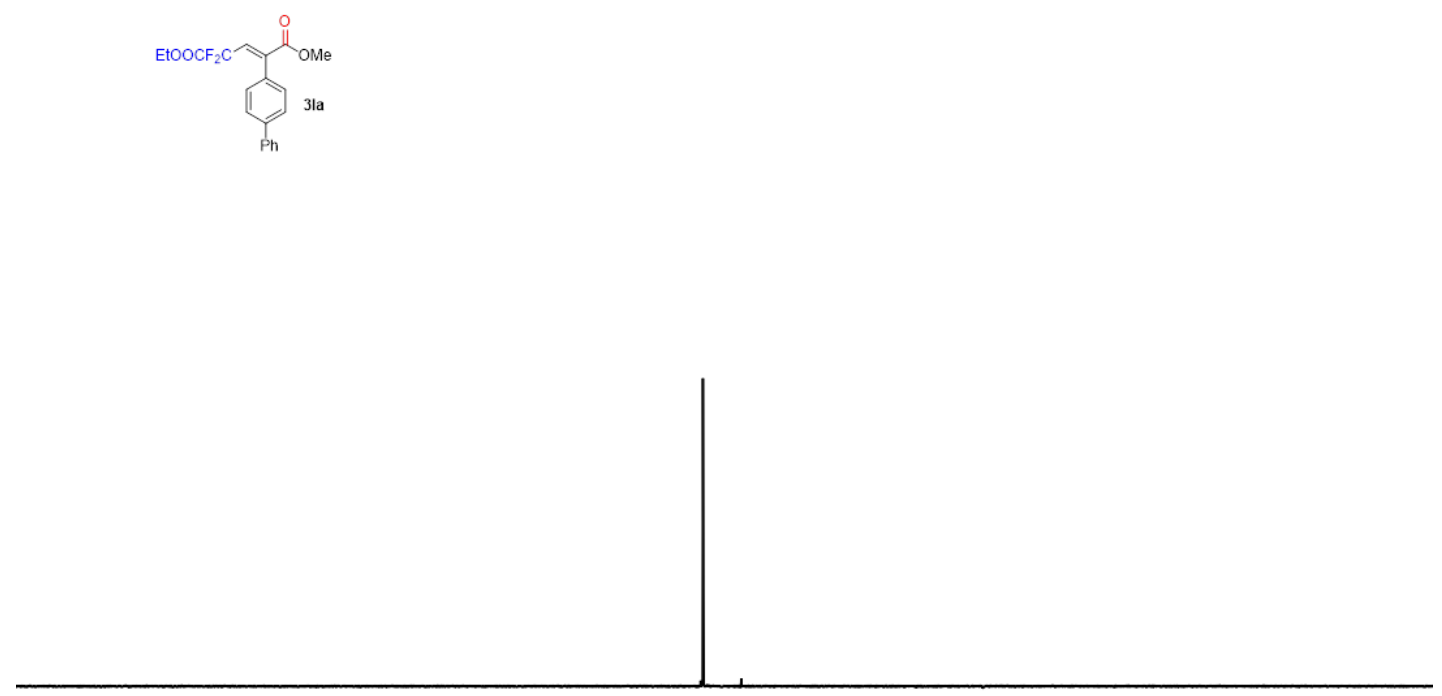

$-20$

$-40$

$-60$

$-80$

ppm $^{-100}$

$-120$

$-140$

$-160$ 


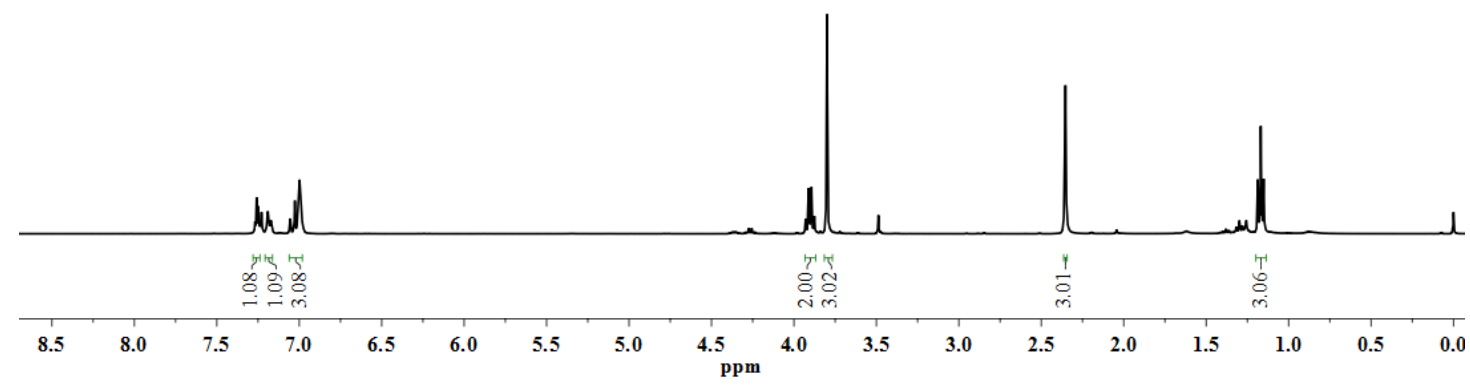

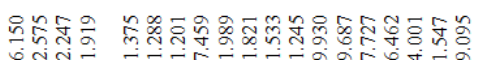

过告守

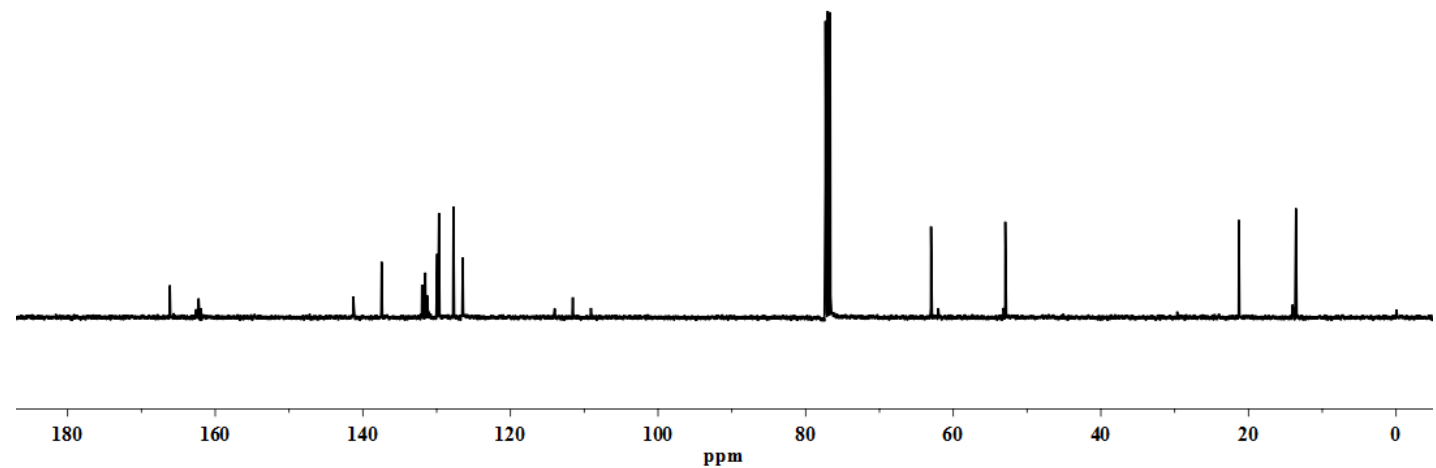


EtoocF 2 Come

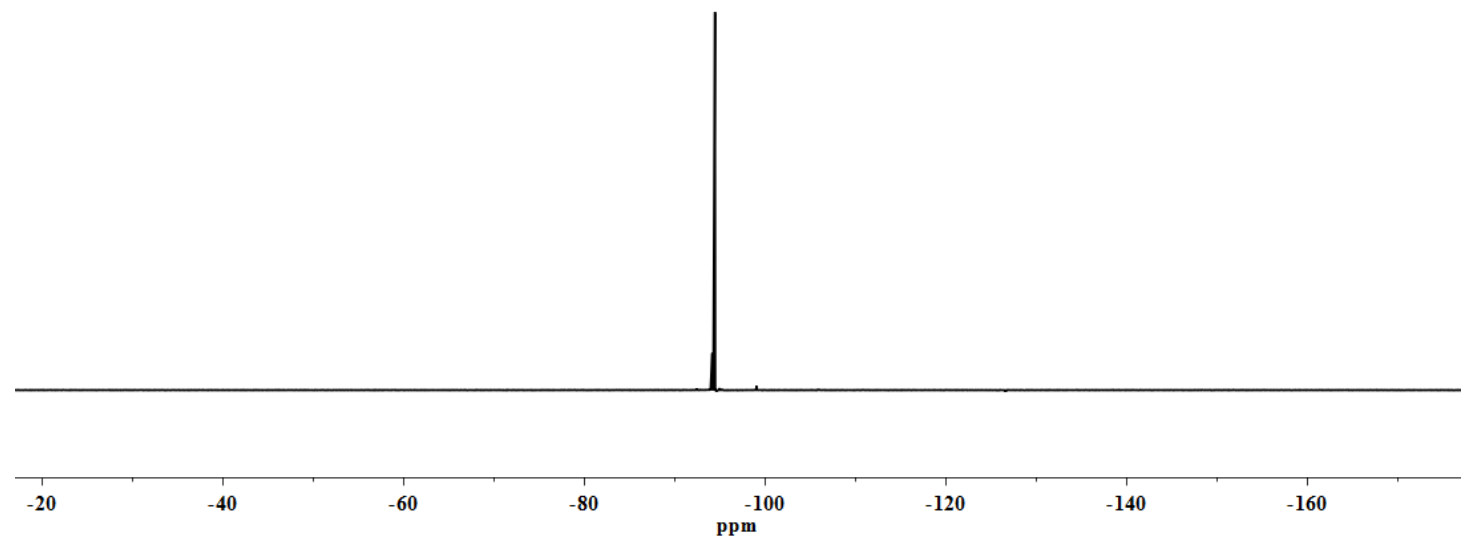

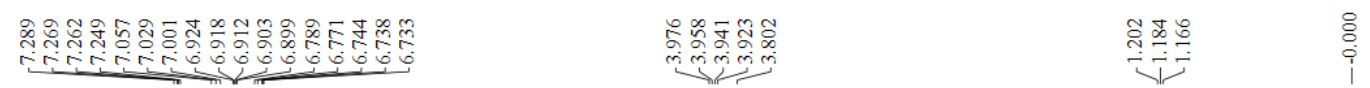
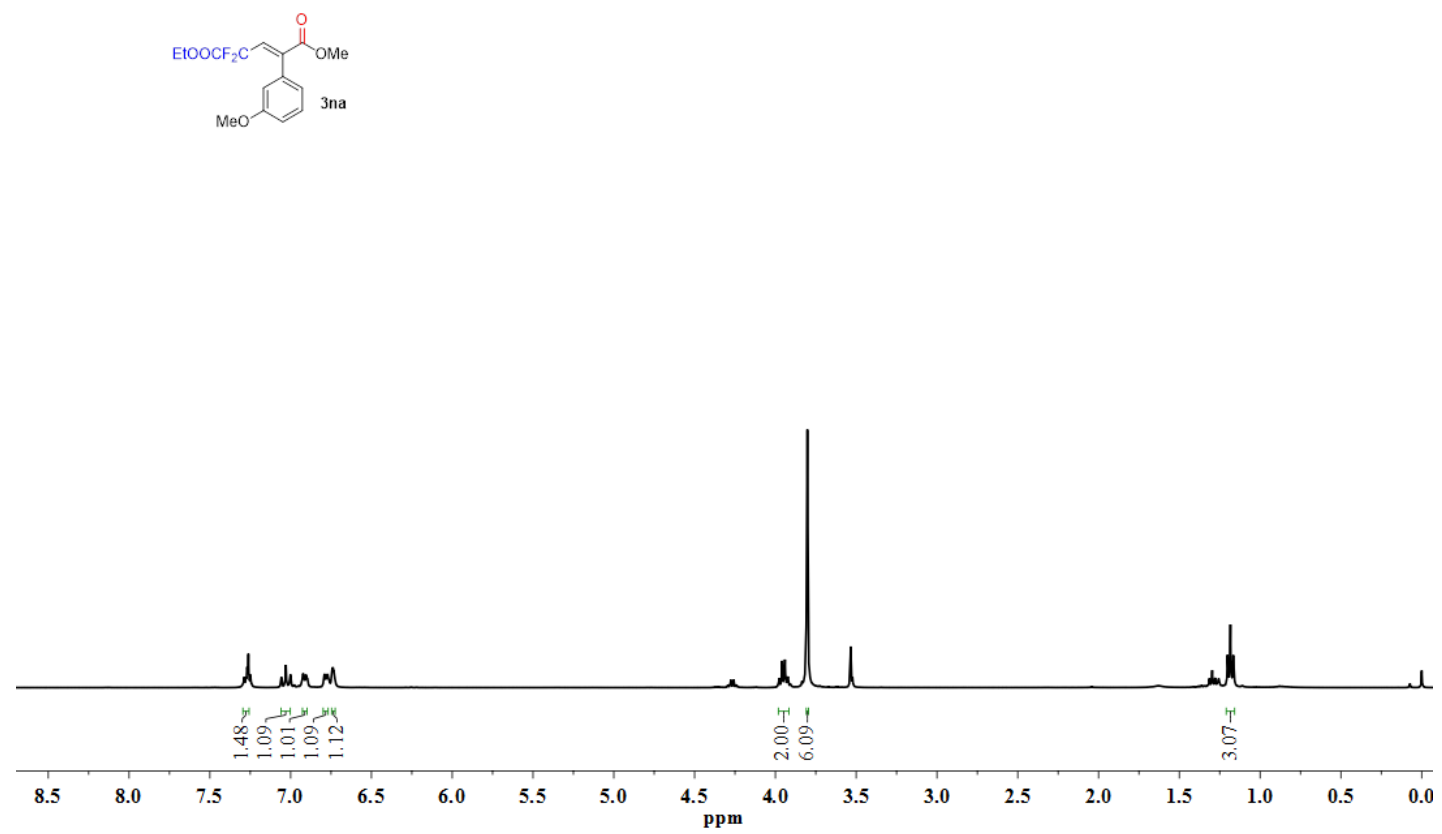


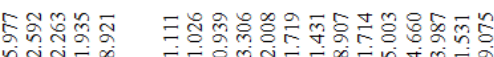

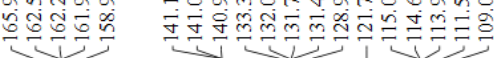

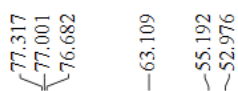

$\stackrel{\infty}{\infty}$

$\mathrm{EtOOCF}_{2} \mathrm{C}$

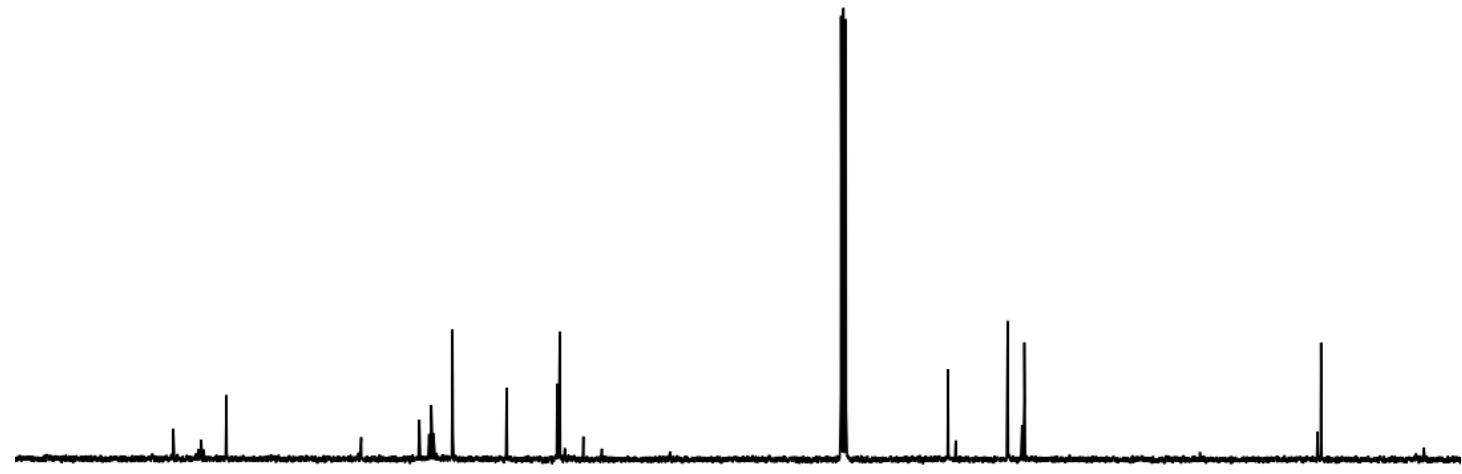

180

160

$40 \quad 120$

100 ppm

$80 \quad 60$

40

$20 \quad 0$

8
i
$i$

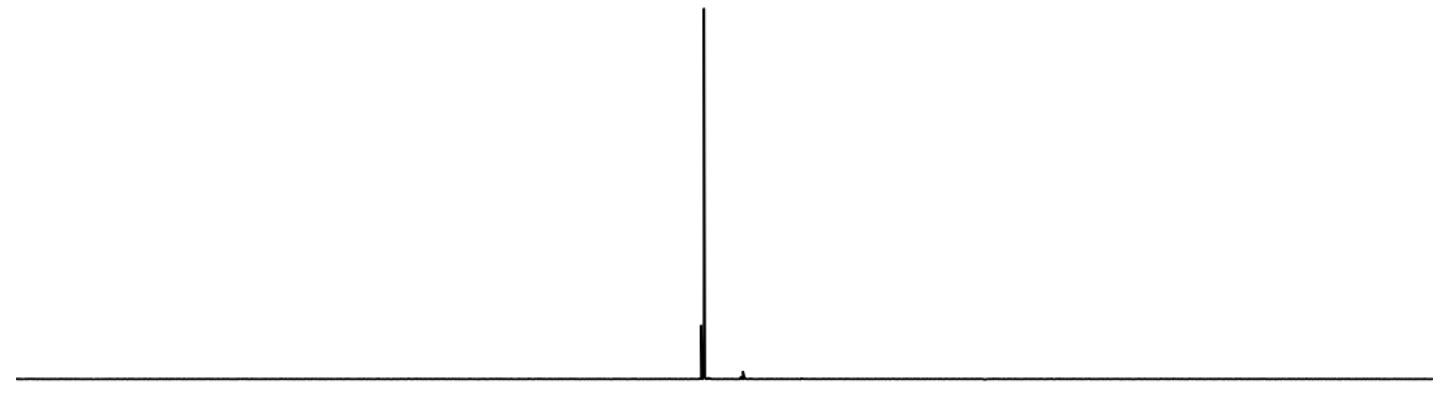

$-20$

$-40$

$-60$

$-80$

-100
ppm

$-120$

$-140$

$-160$ 

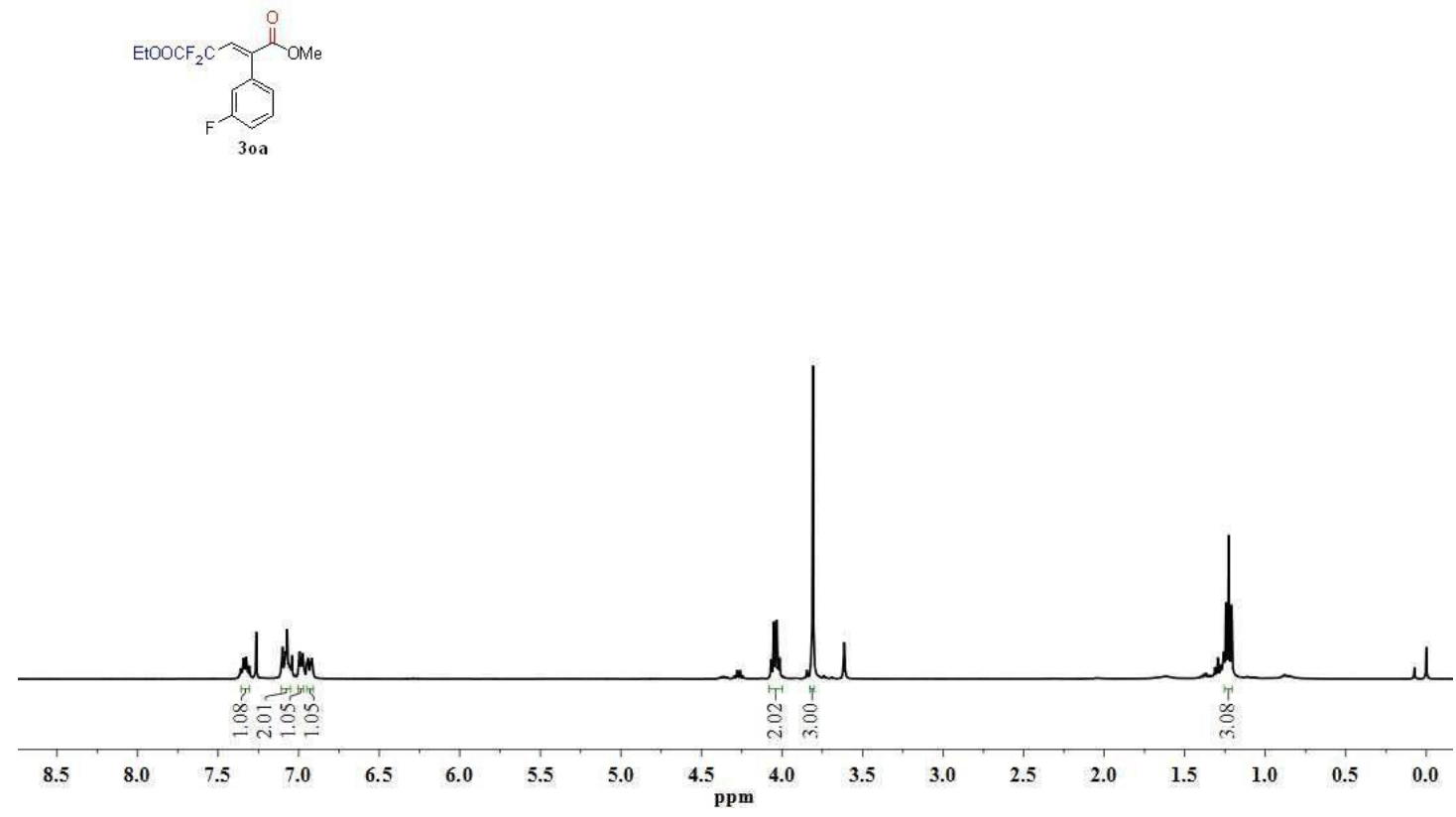

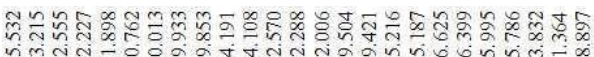

ทู่

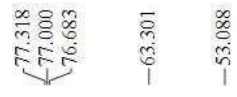<smiles>CCOC(=O)CC=C(C(=O)OCC)c1cccc(F)c1</smiles>

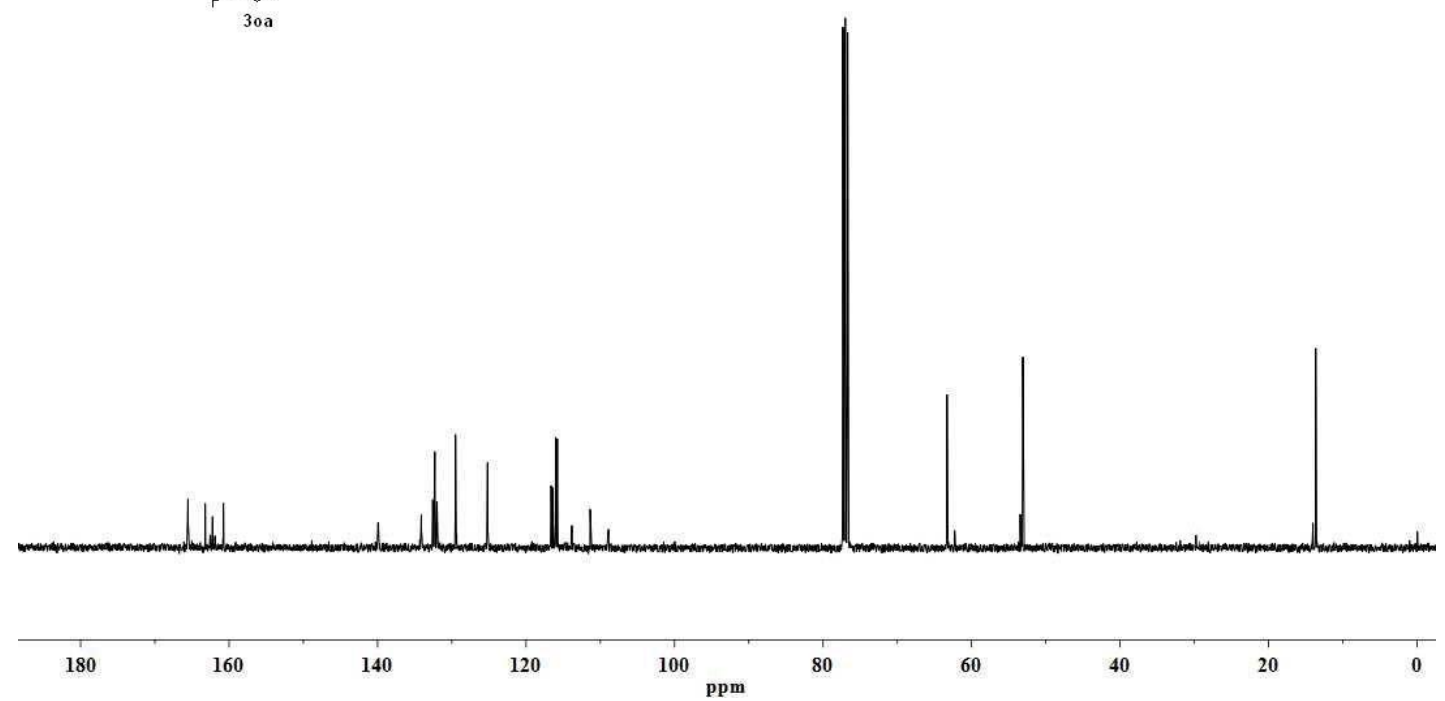




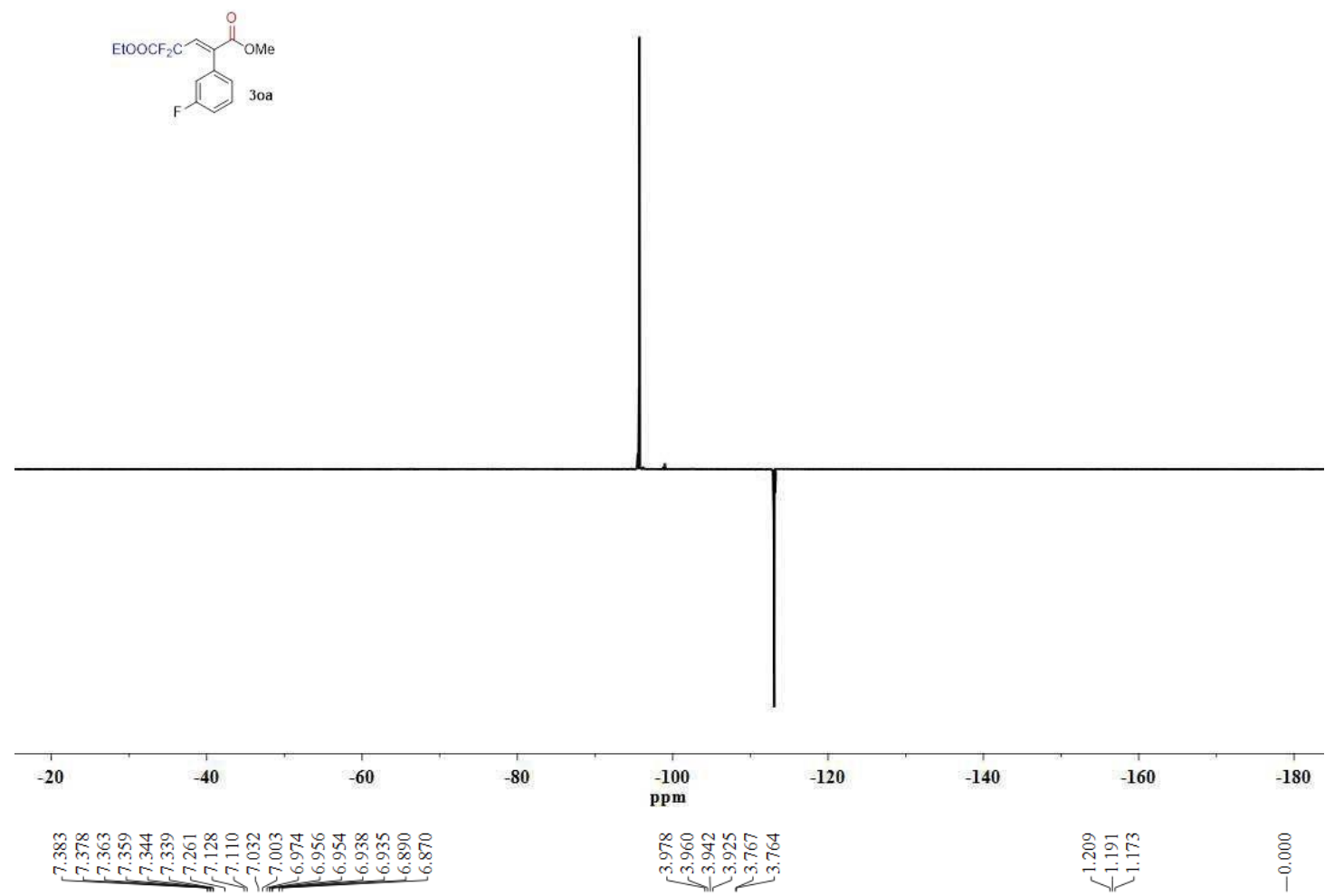

$$
\text { EtoocF } 2 \text { C }
$$

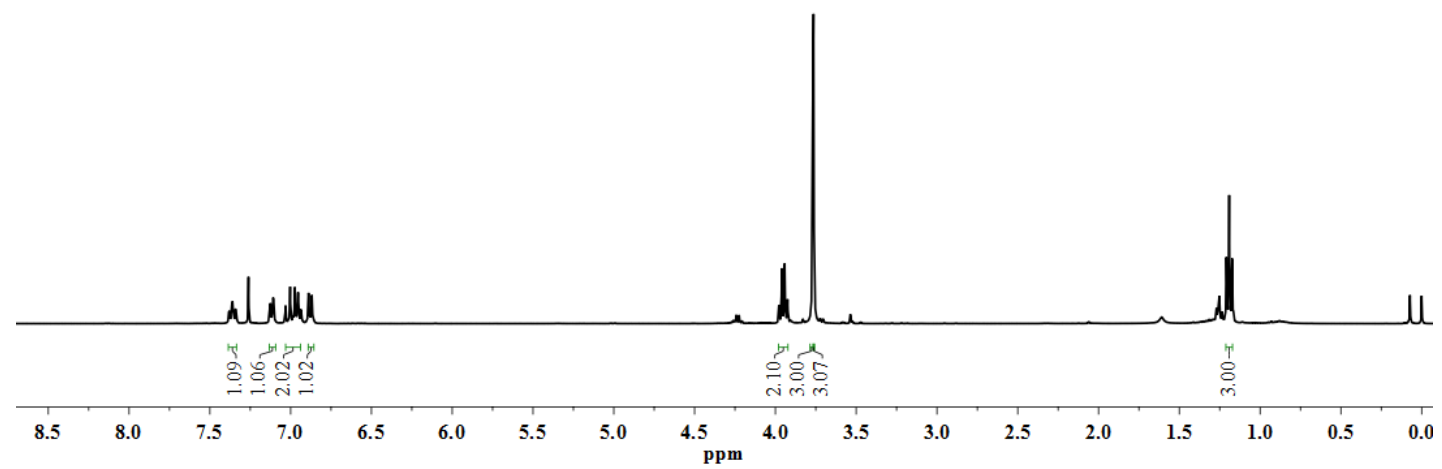




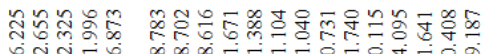

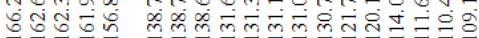

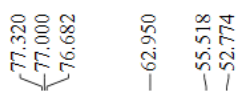

EtoOCF 2 C OMe

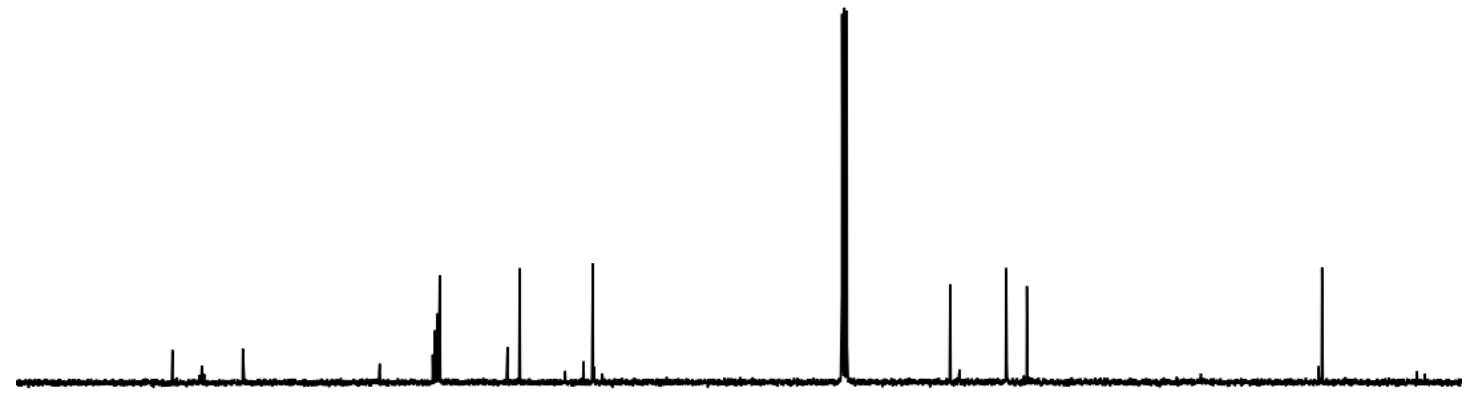

180

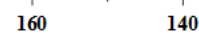

$140 \quad 120$

$100 \quad 80$

60

40

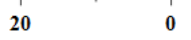

m.
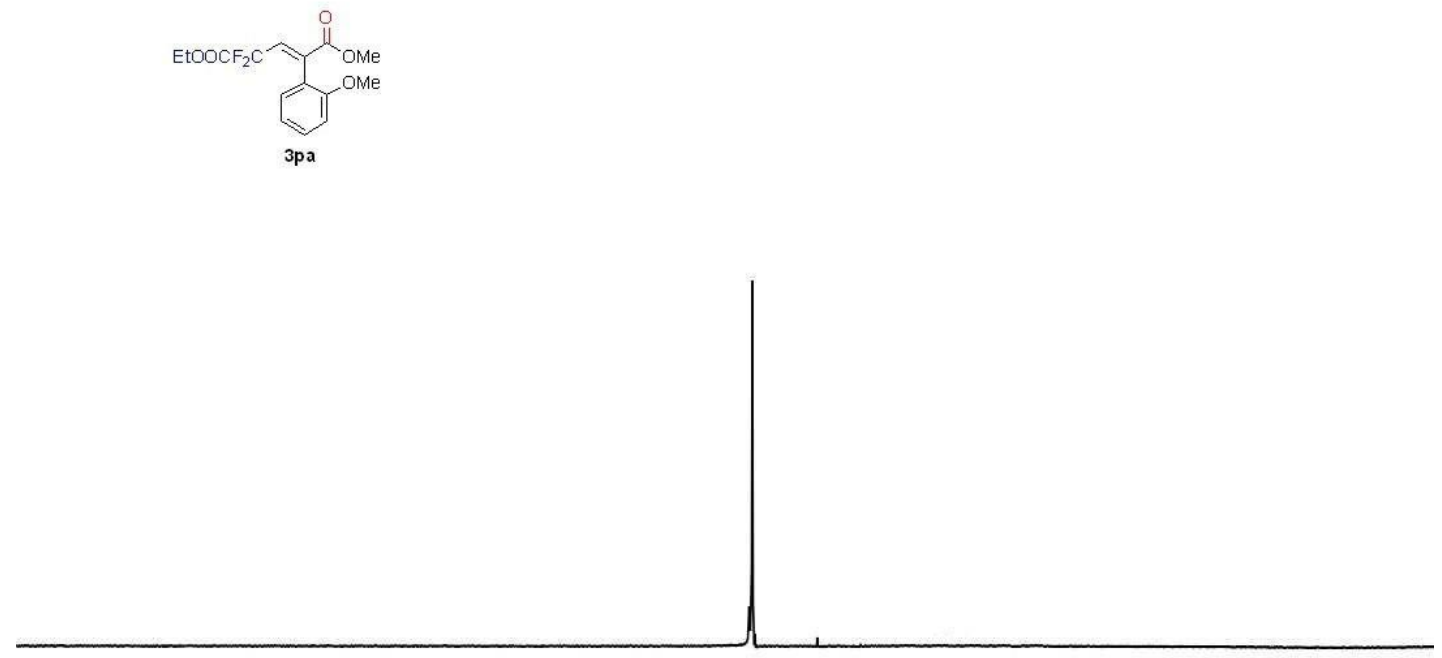

$-20$

$-40$

$-60$

$-80$

ppm $^{-100}$

$-120$

$-140$

$-160$ 


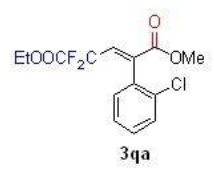

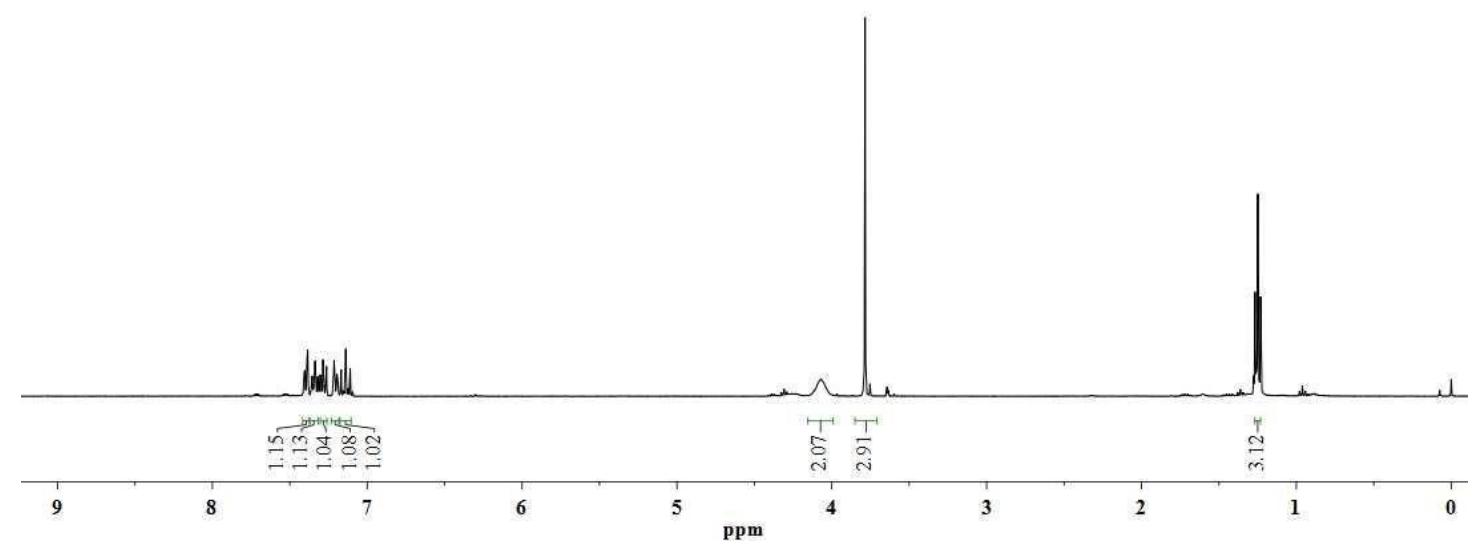

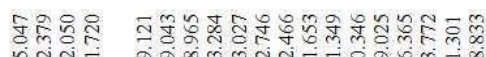

vi⿺辶寸 m

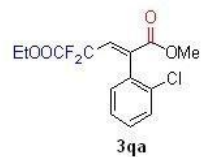

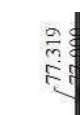

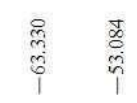
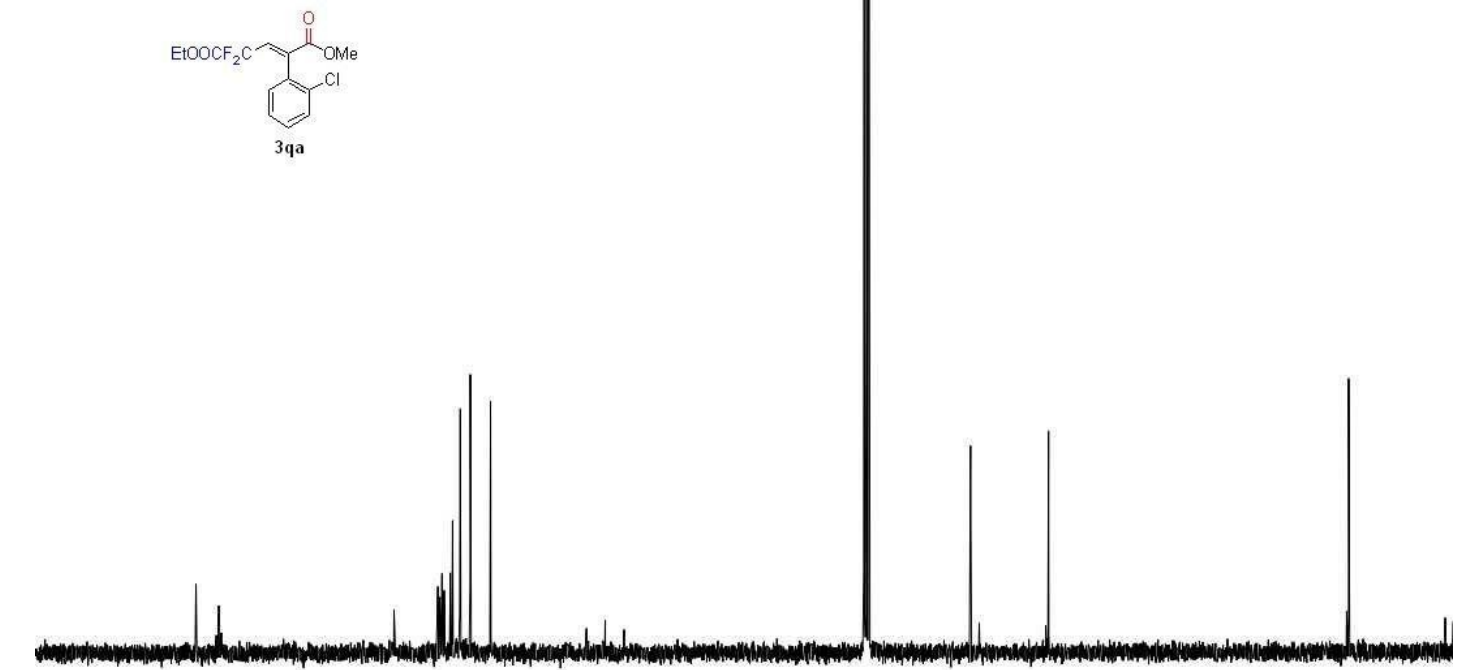


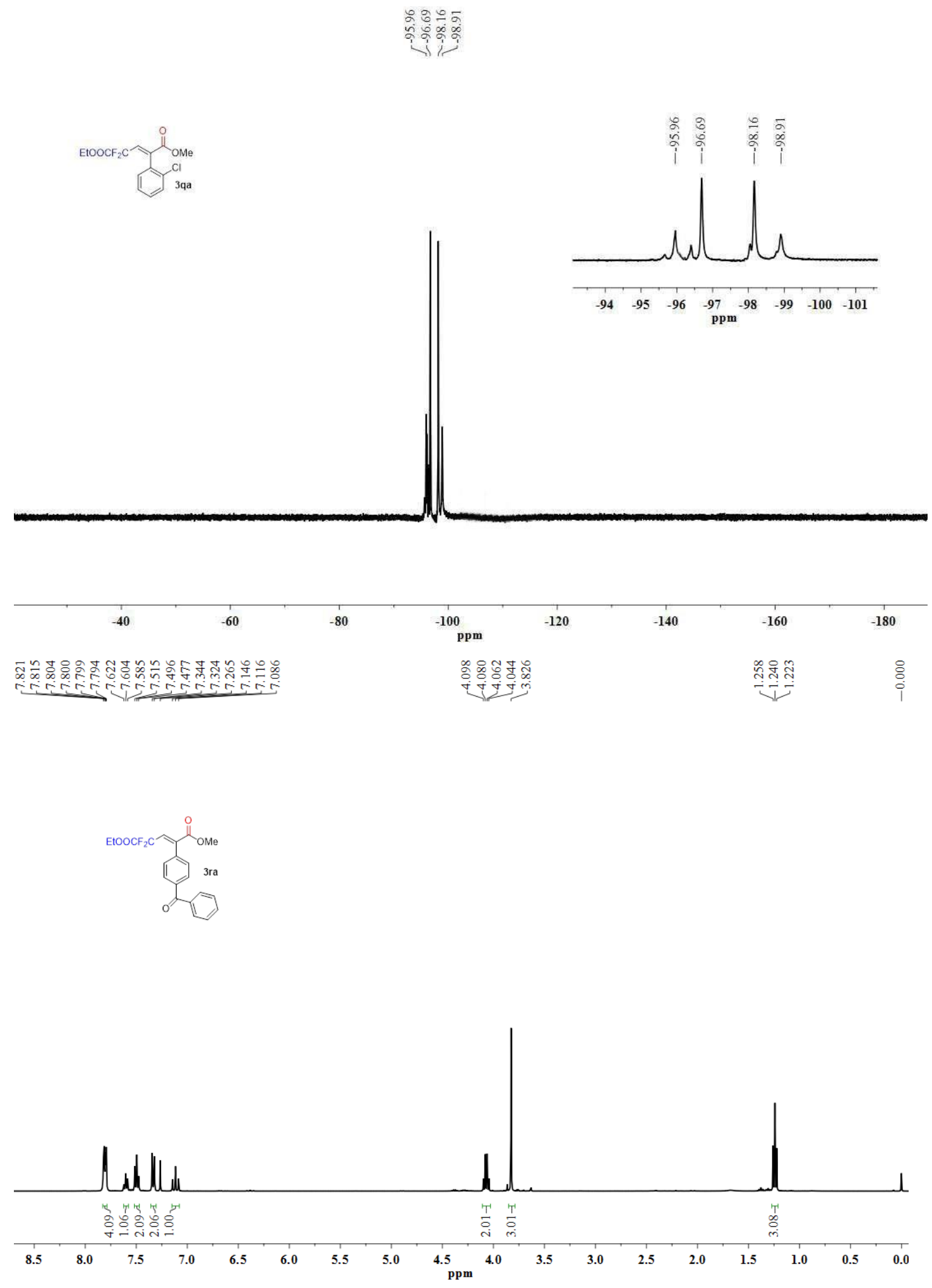



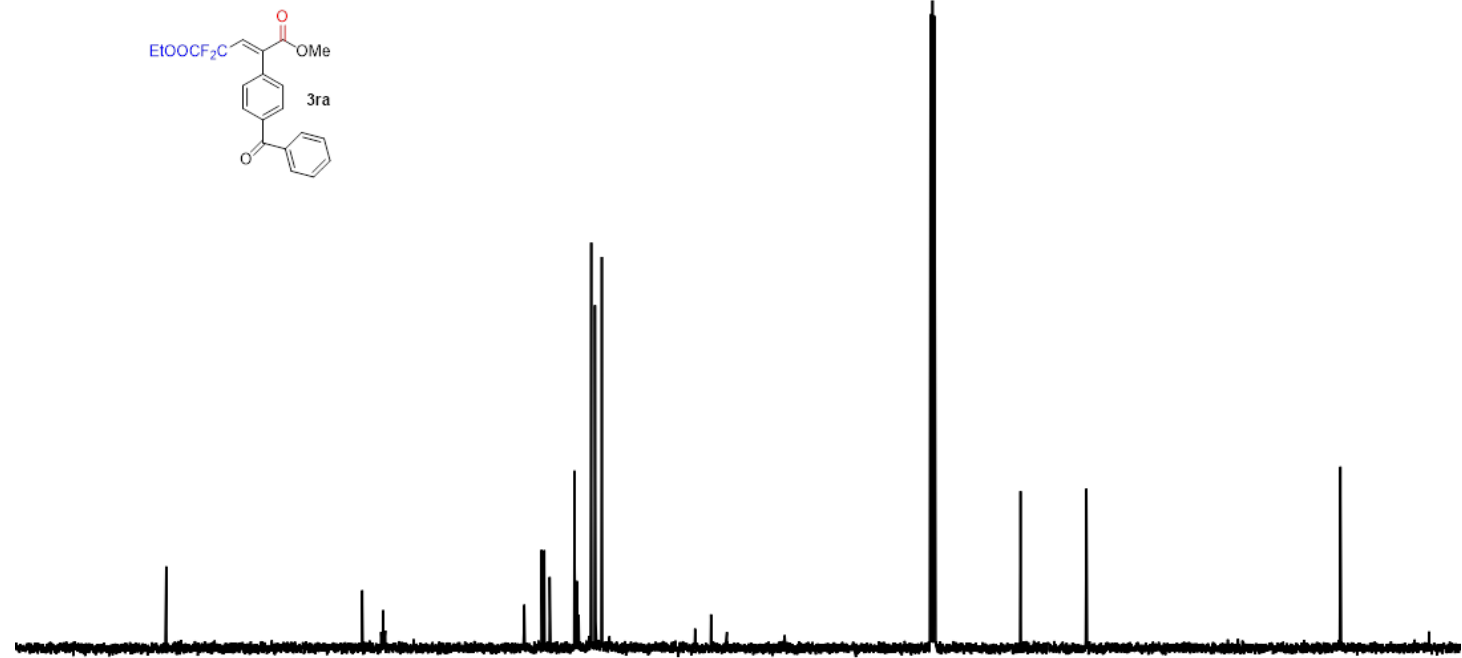

200
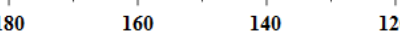
$20 \quad$ ppm

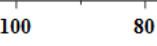

80 60 40

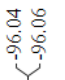
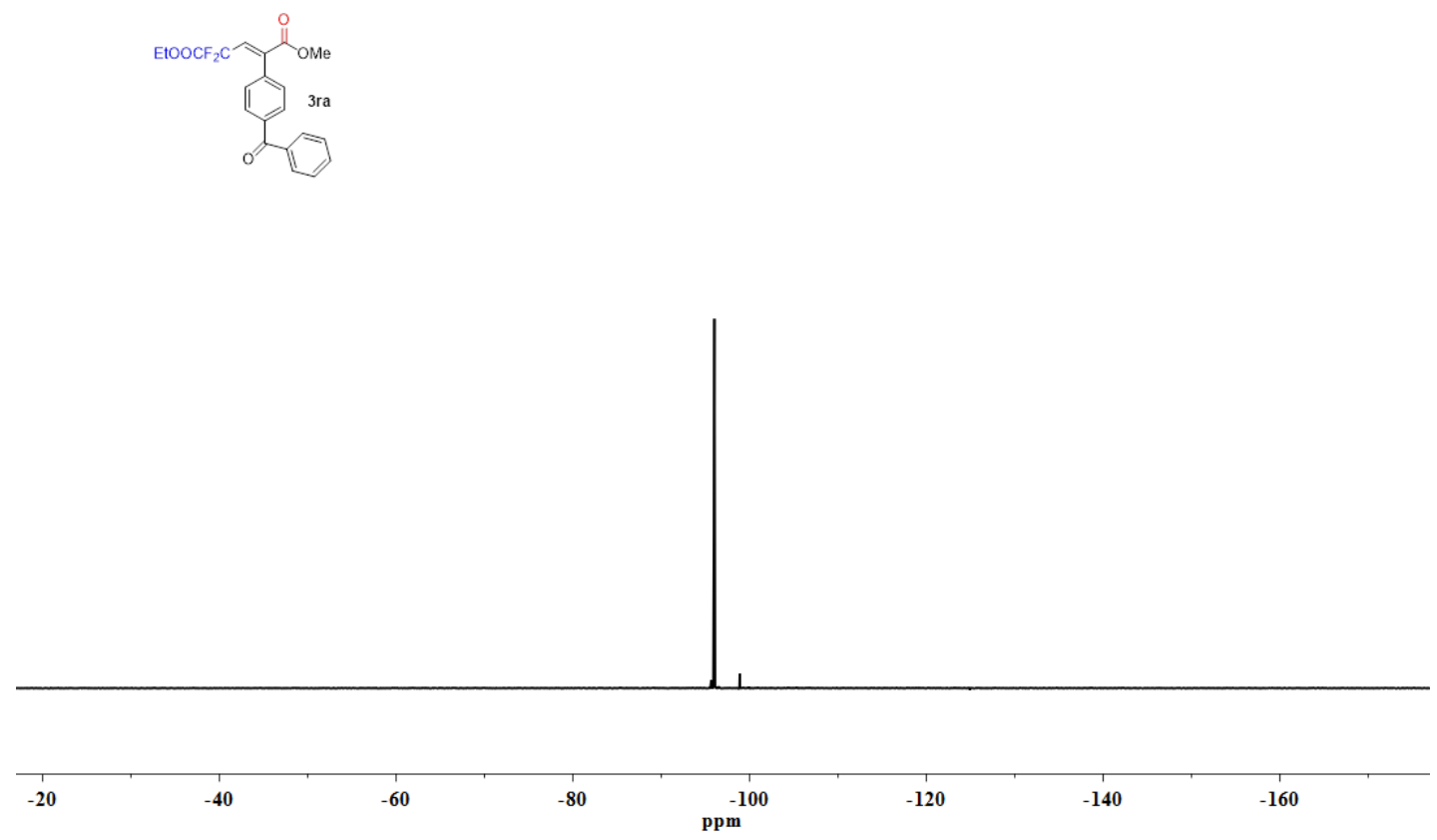

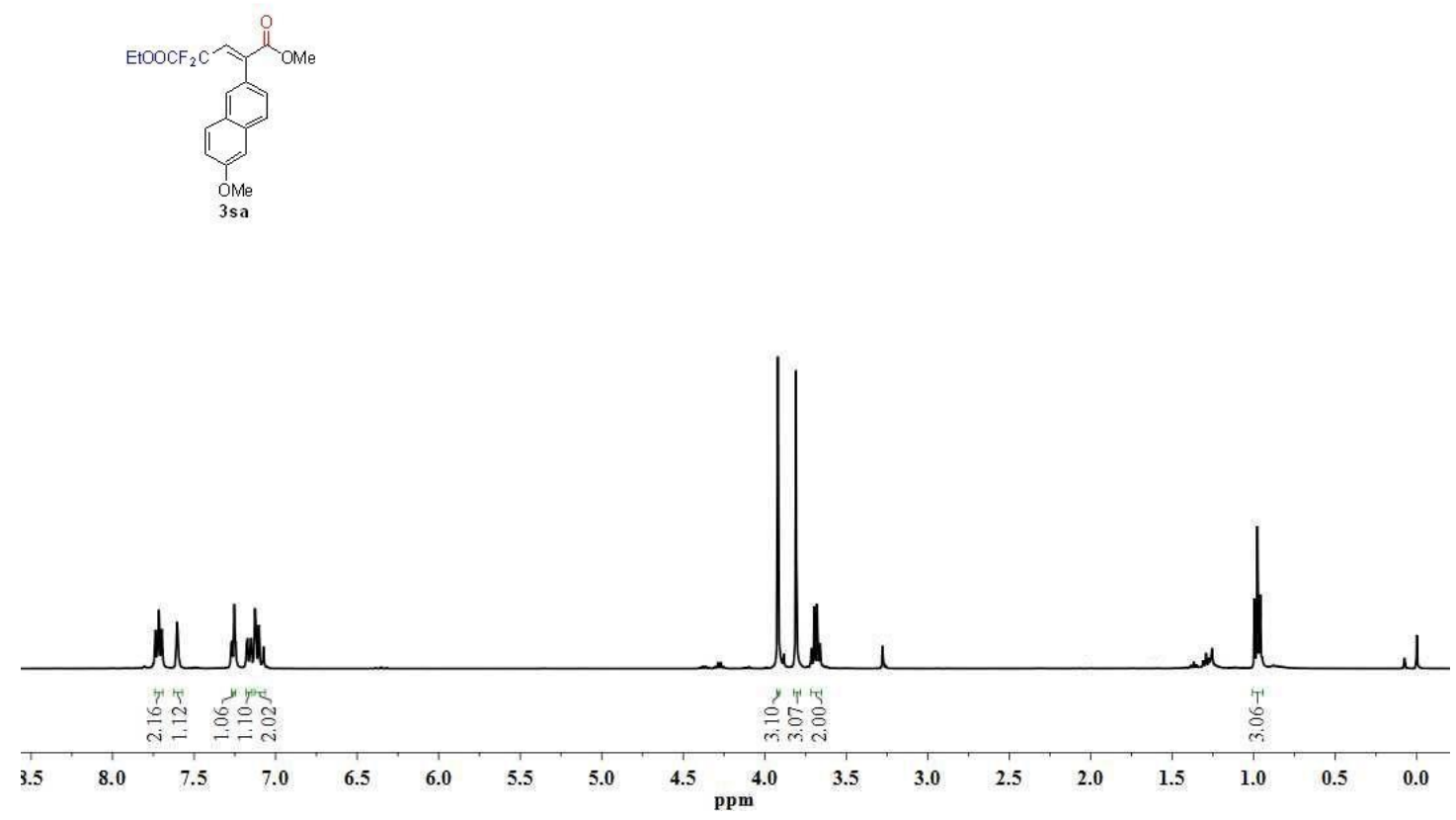

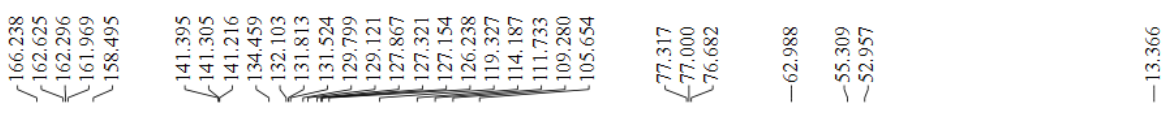

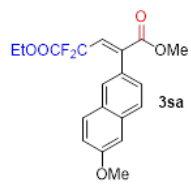

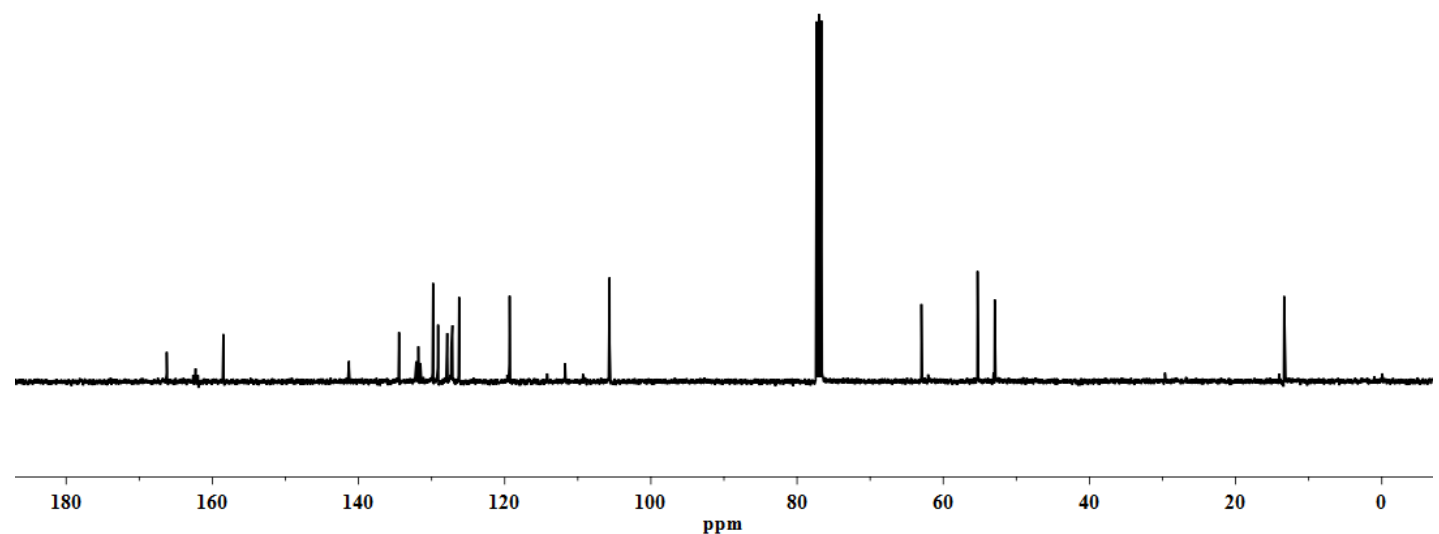



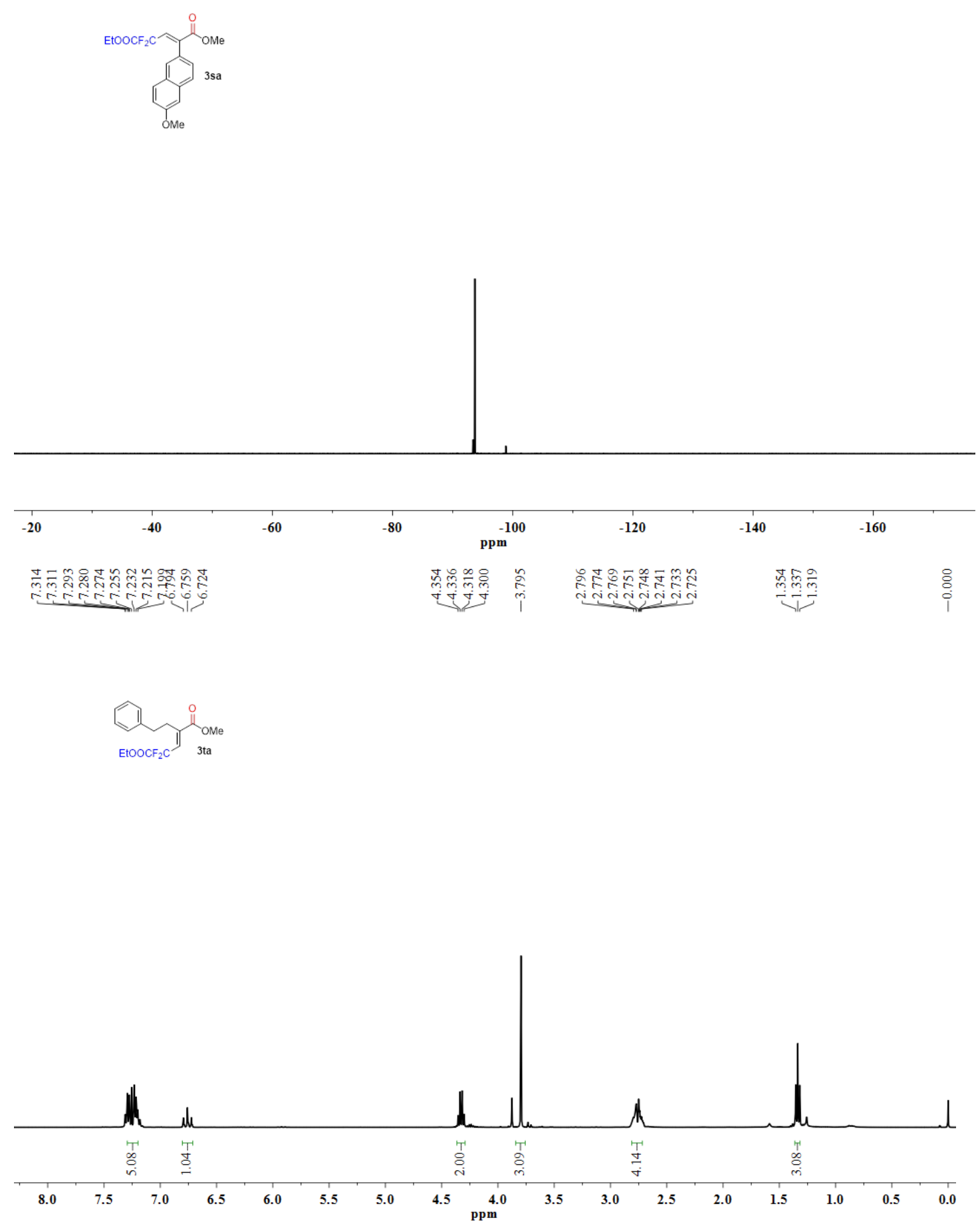


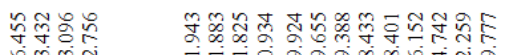

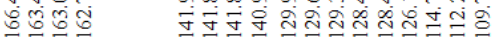

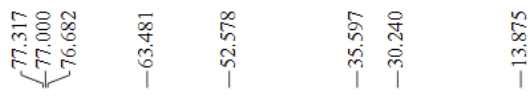
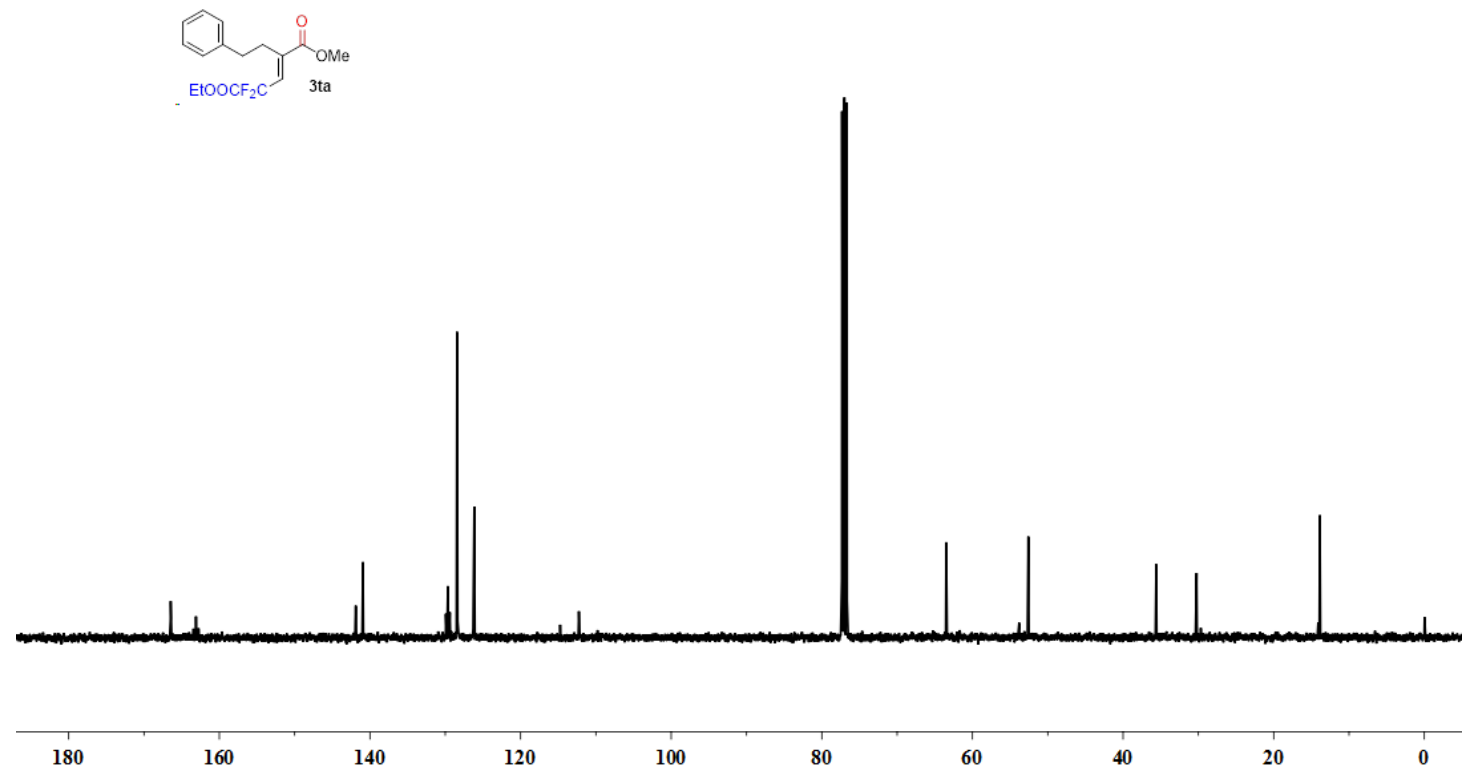

$100 \quad$ ppm

80

60

40

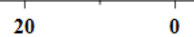

$\frac{\sqrt{3}}{\frac{1}{8}}$
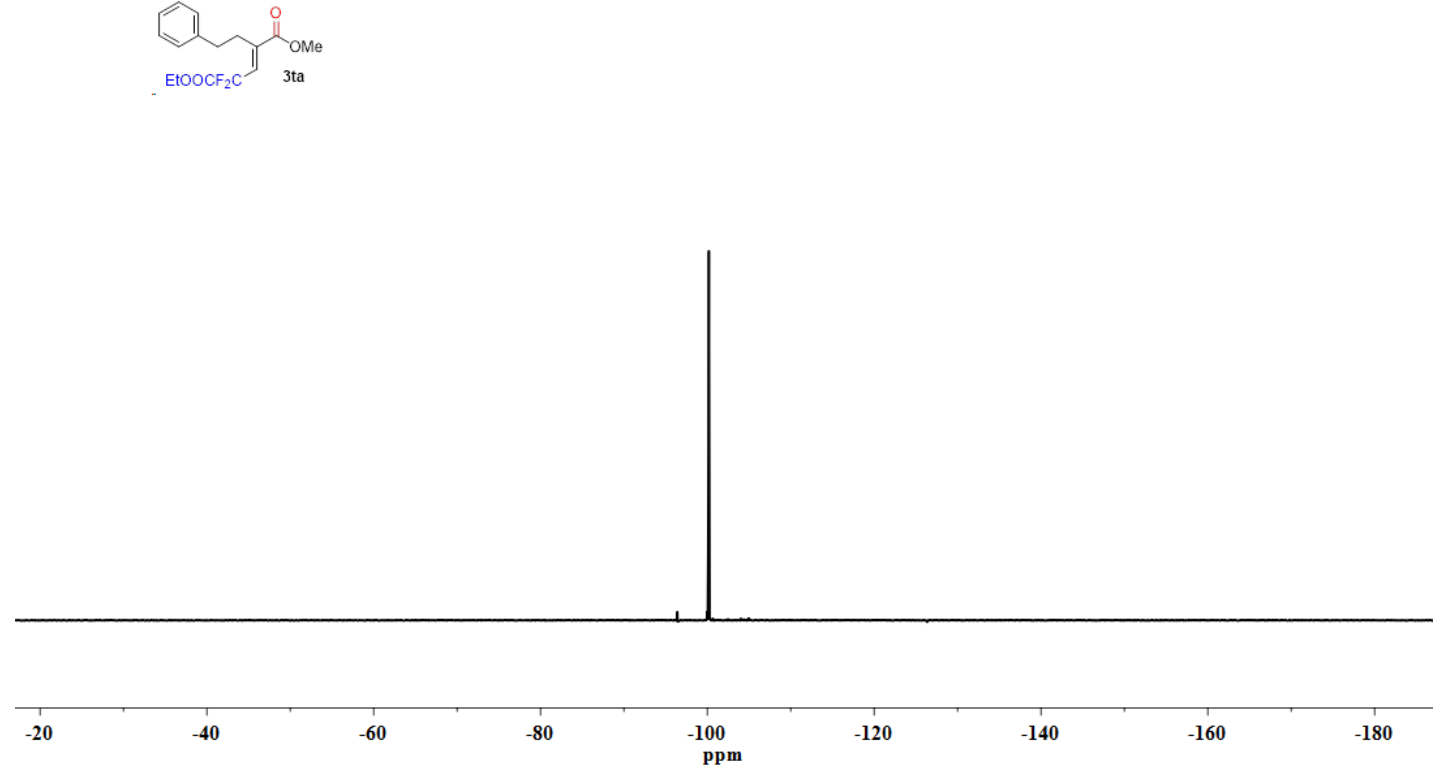

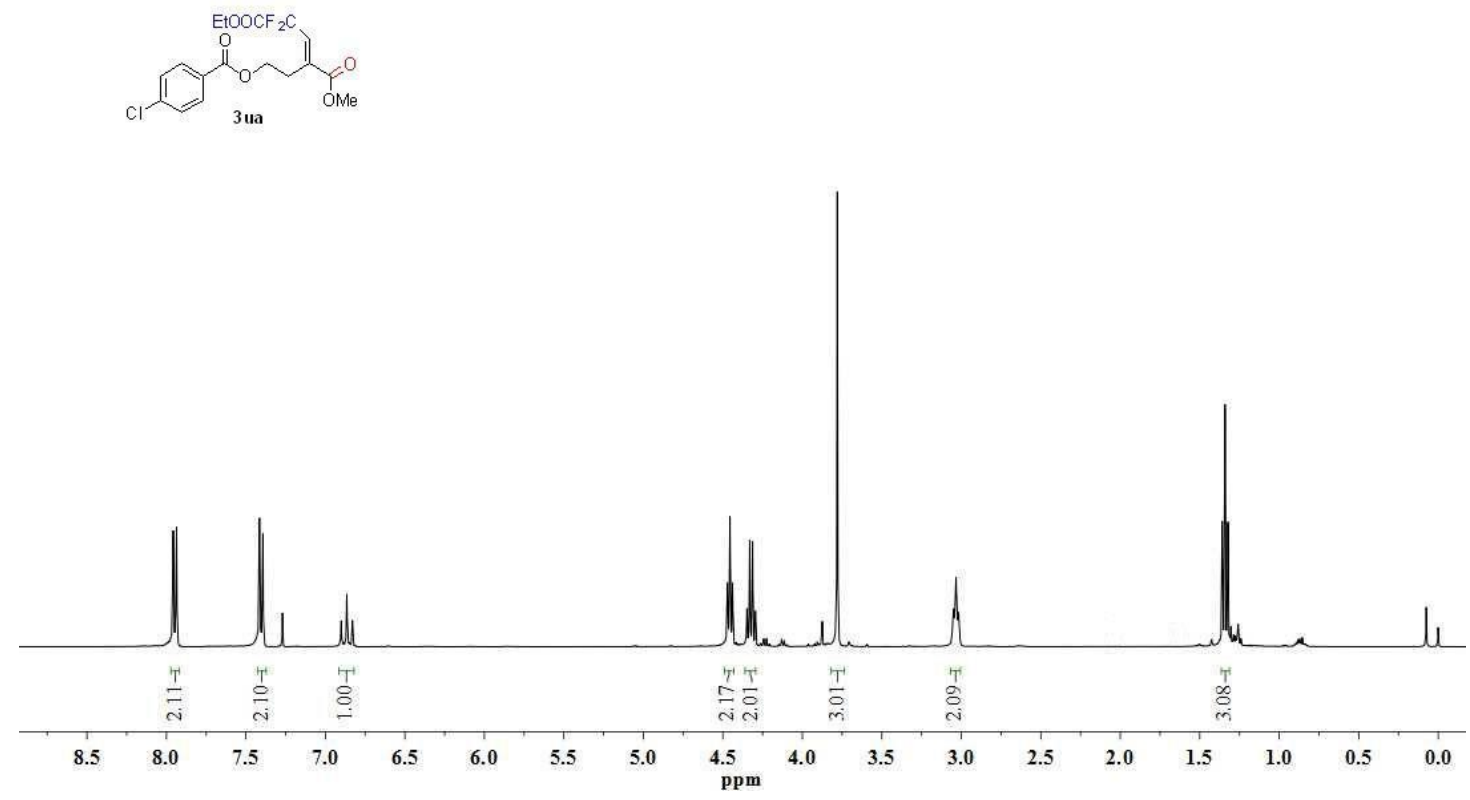

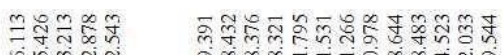

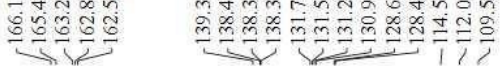

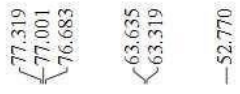

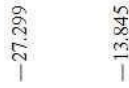
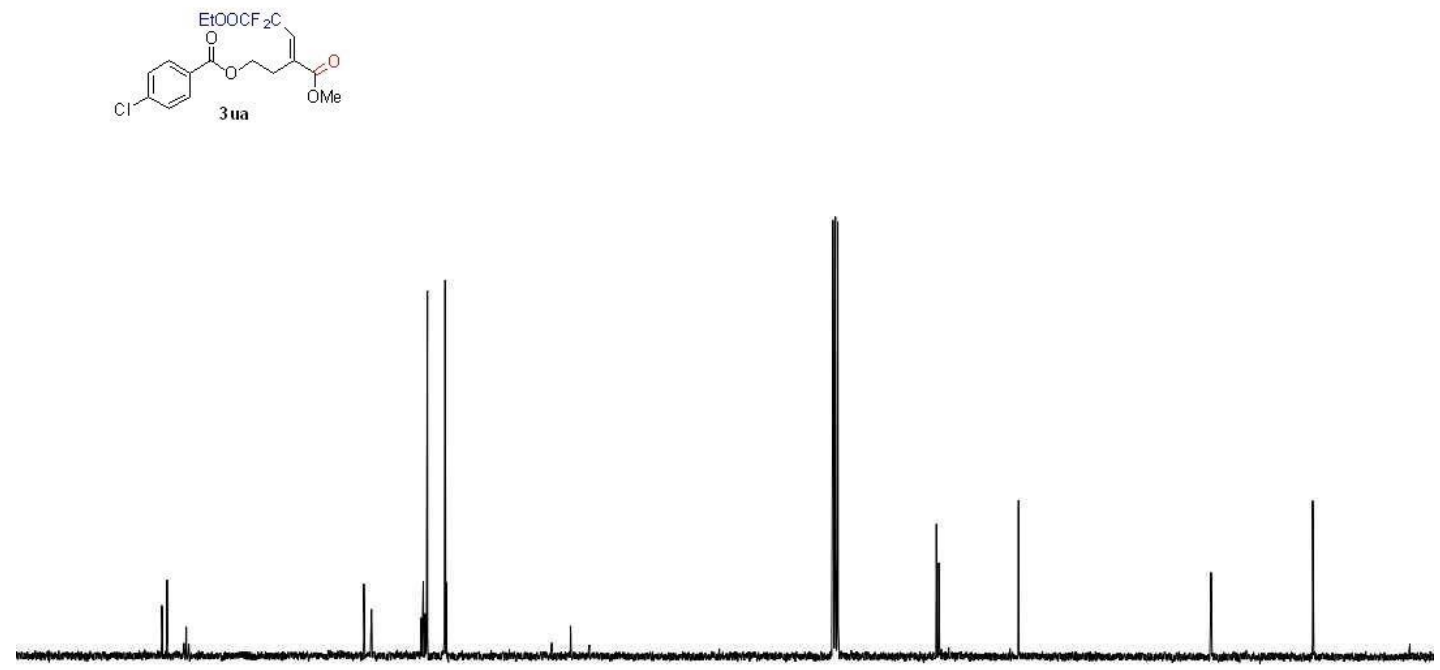

180

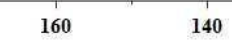

140

120

100

80

60

40

20

0 
3ua OMe

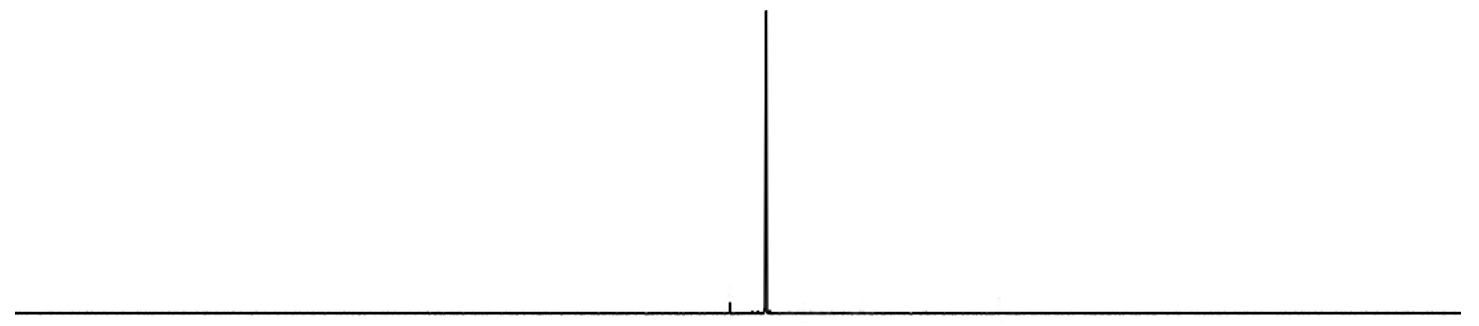

3va

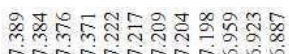

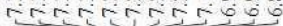

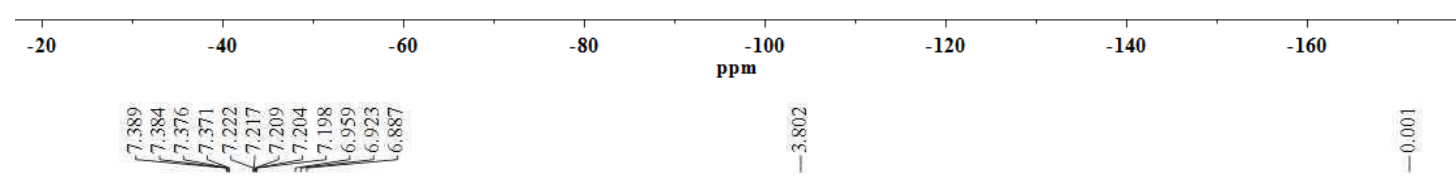

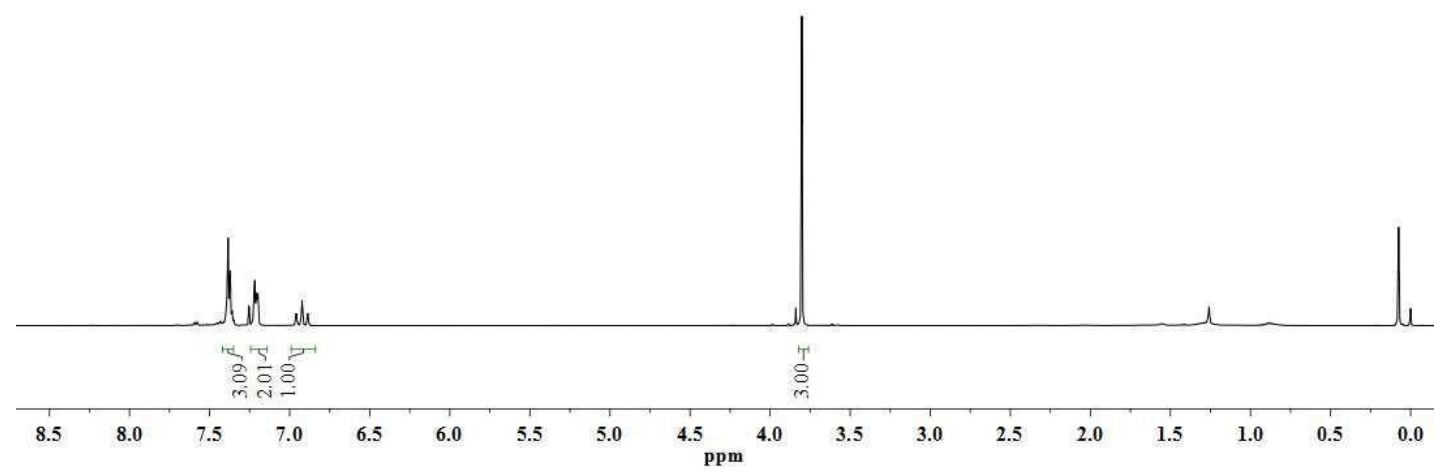



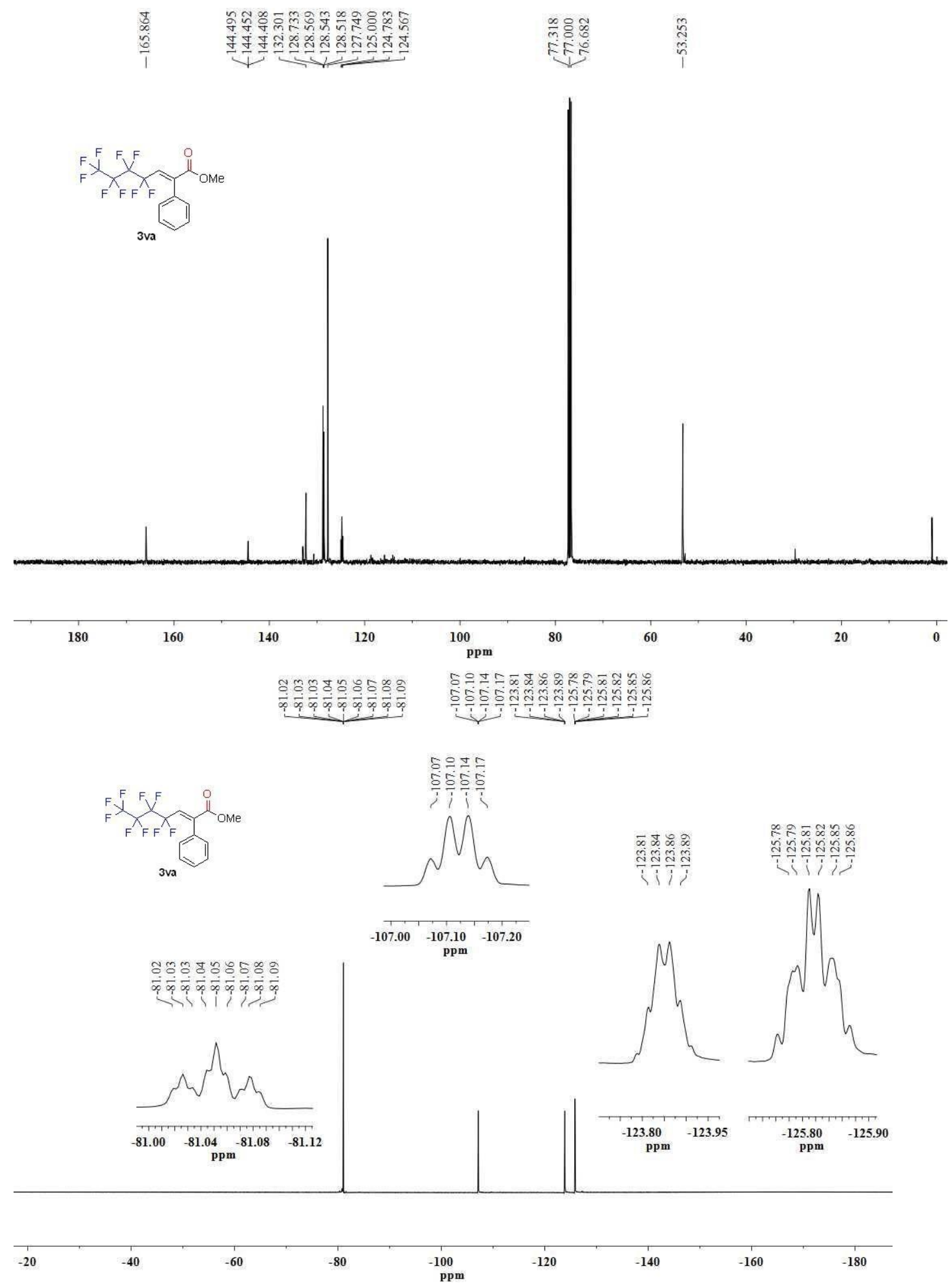

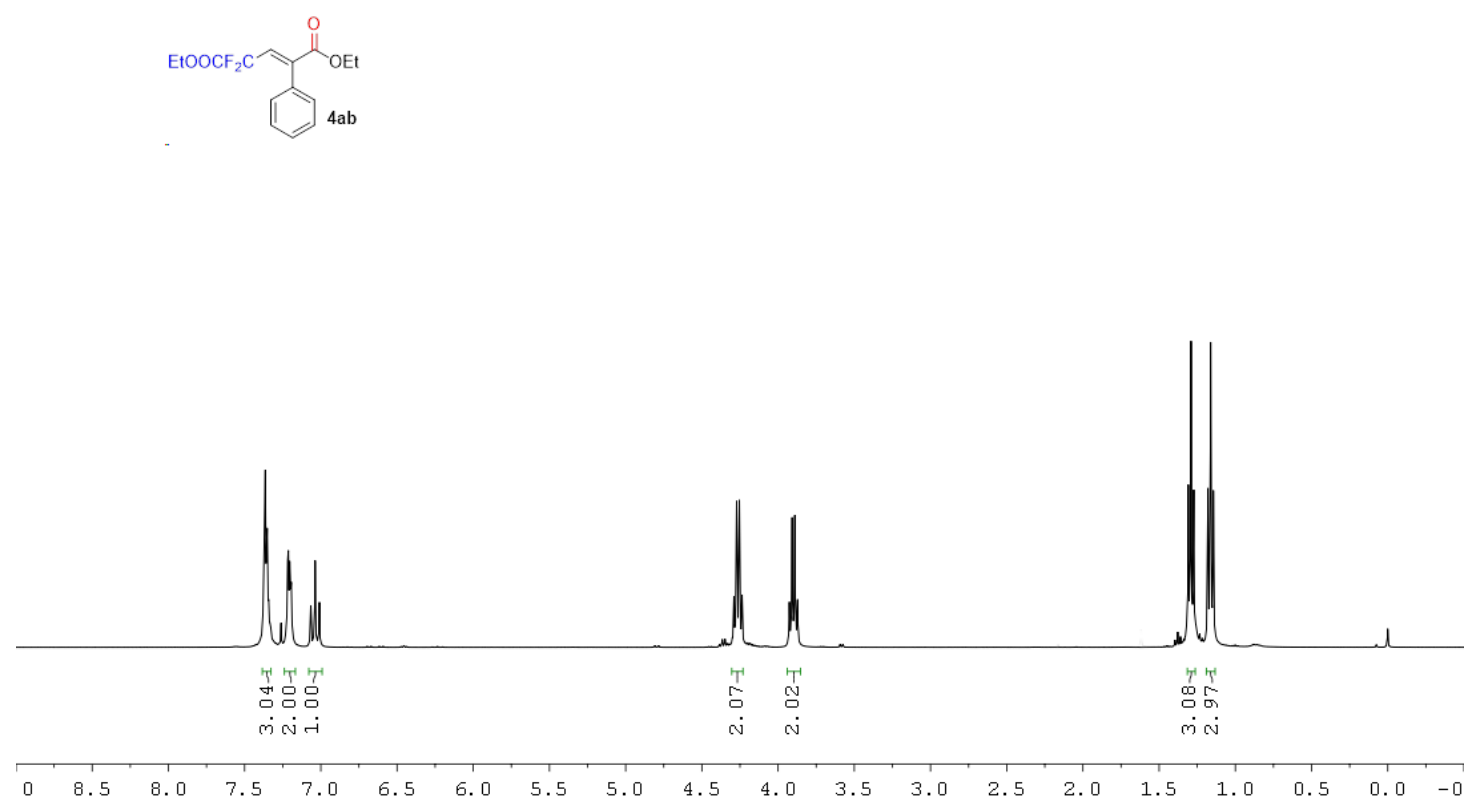

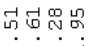

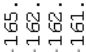

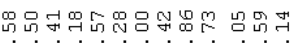

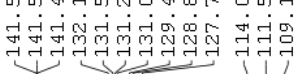

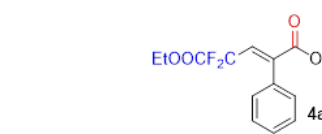

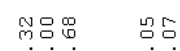

然
돈ำ

सुल

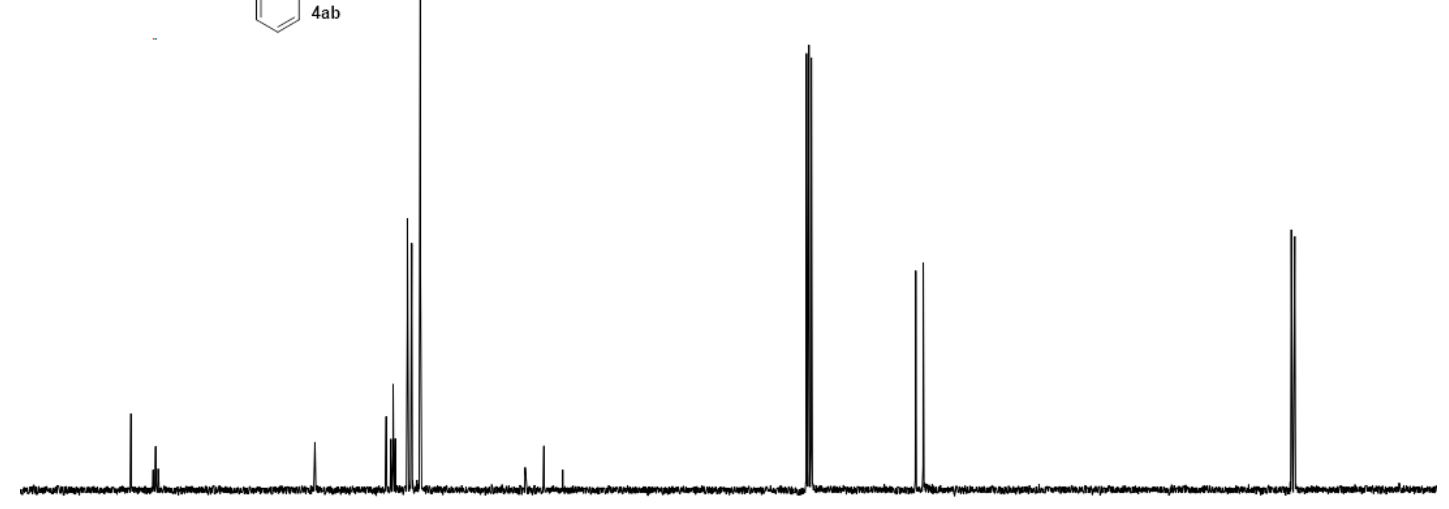

$\begin{array}{llllllll}170 & 160 & 150 & 140 & 130 & 120 & 110 & 100\end{array}$

9080

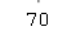

30 


$$
\text { EtOOCF } 2 \text { C }
$$
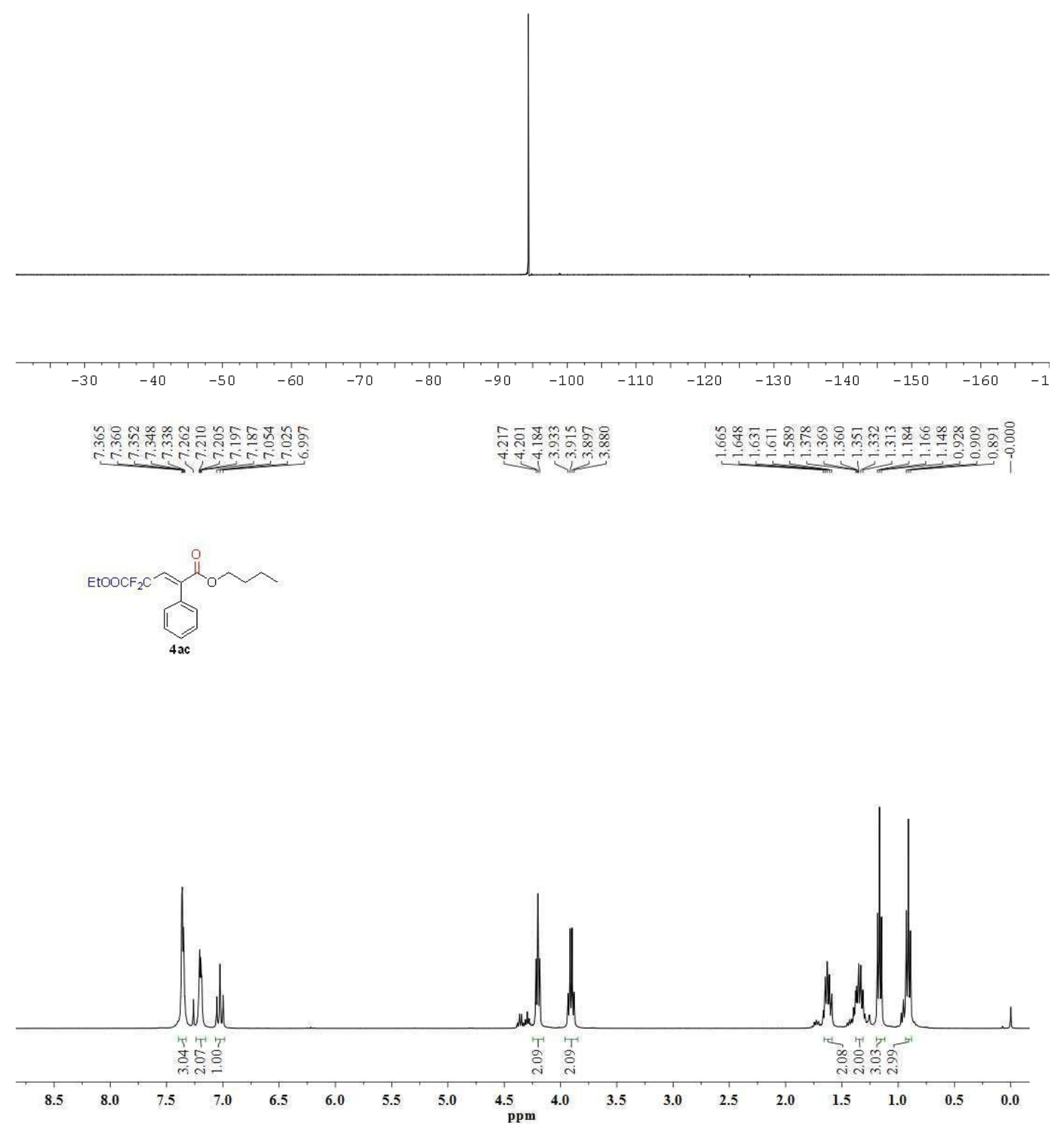


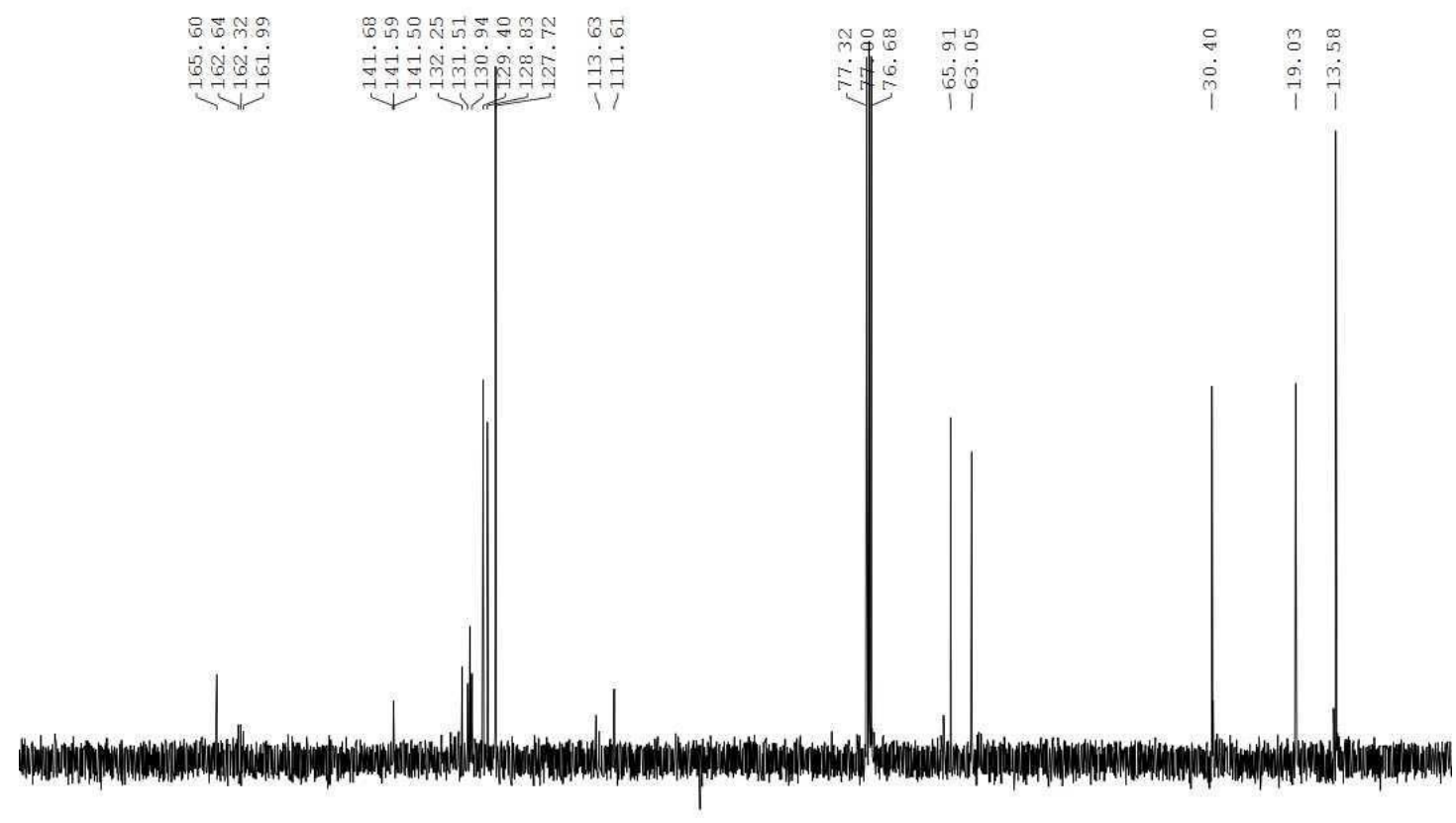

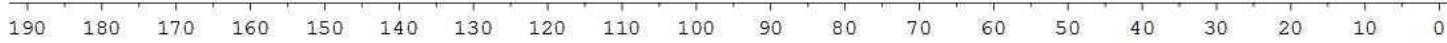
영 $\mathrm{EtOOCF}_{2} \mathrm{C}$

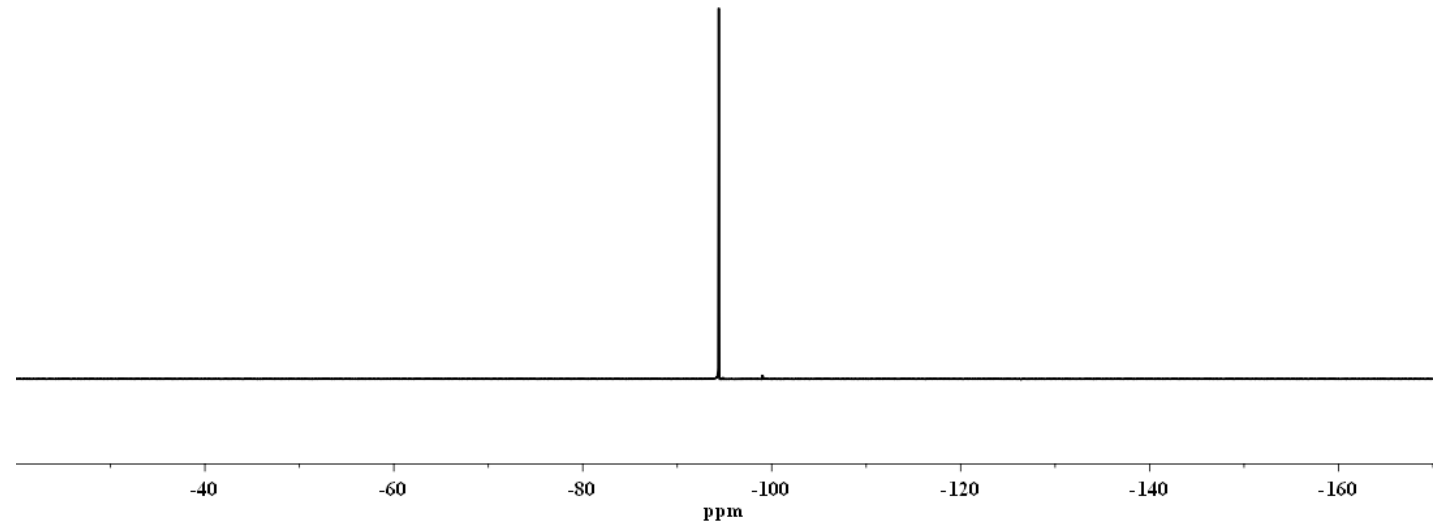



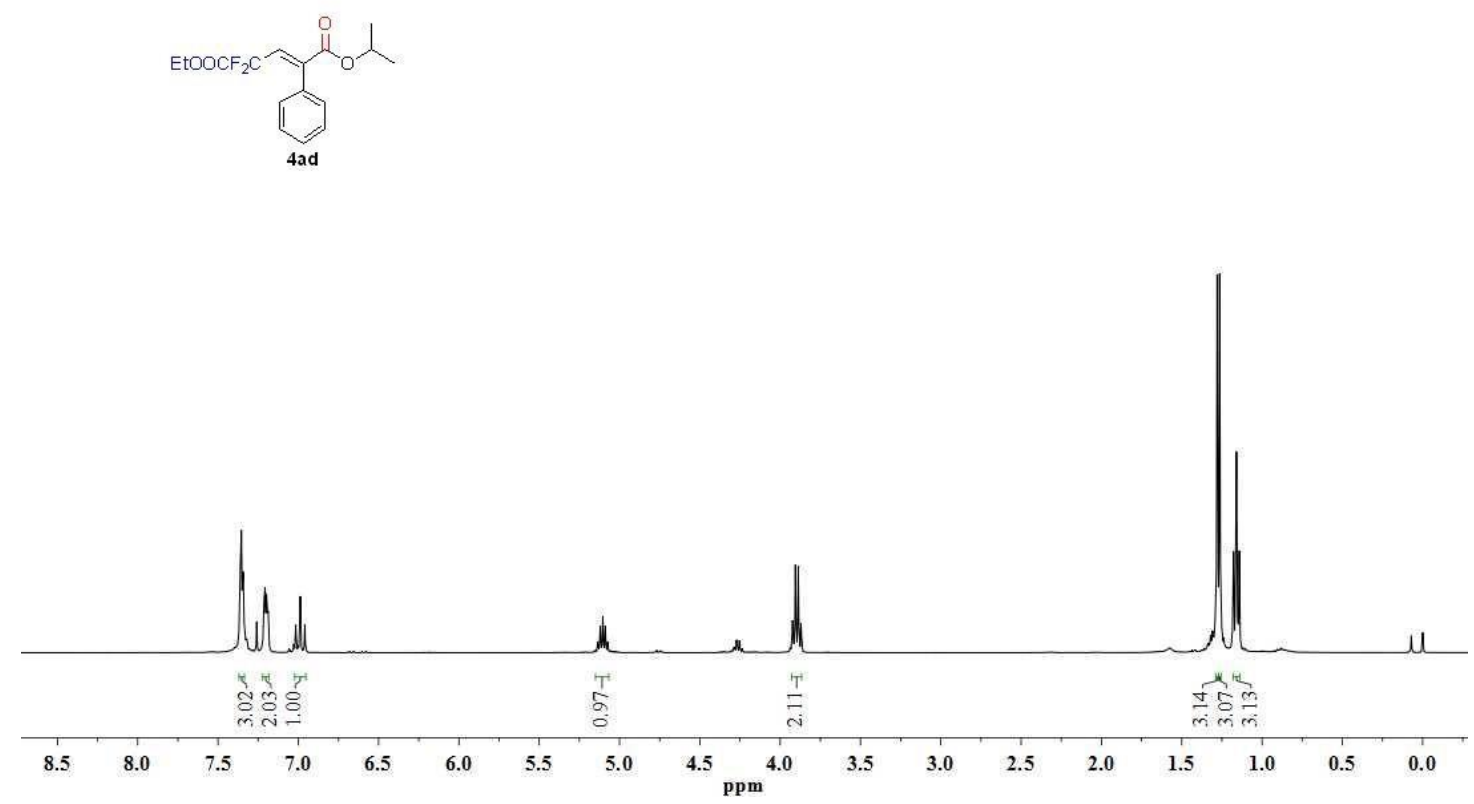

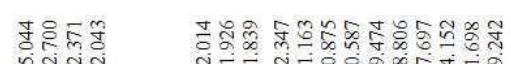

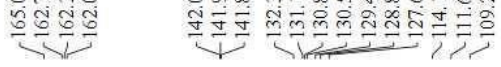

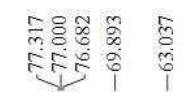

$\begin{array}{ll}\text { ते } & \stackrel{\infty}{0} \\ \text { iे } & \frac{m}{1}\end{array}$

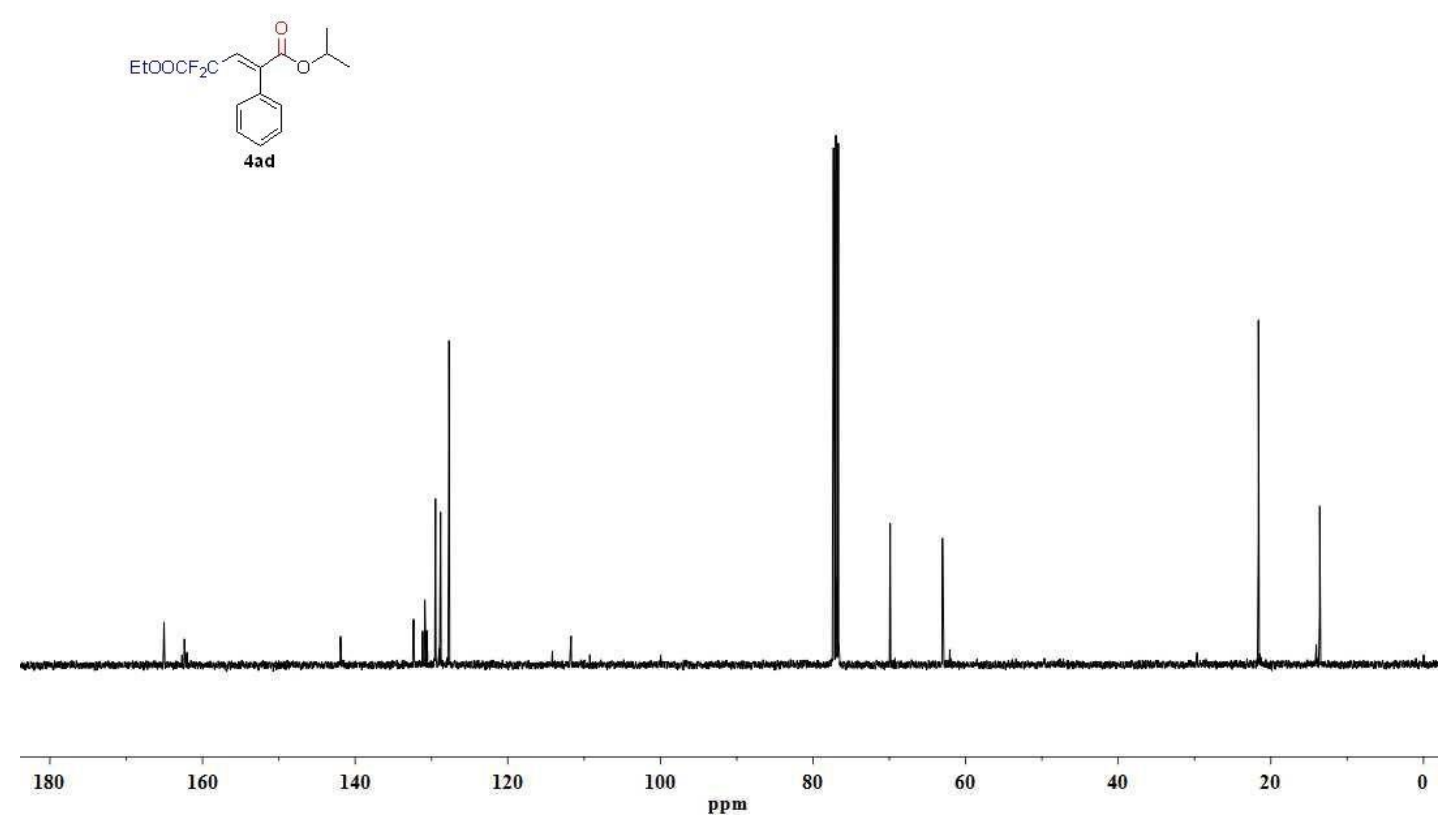



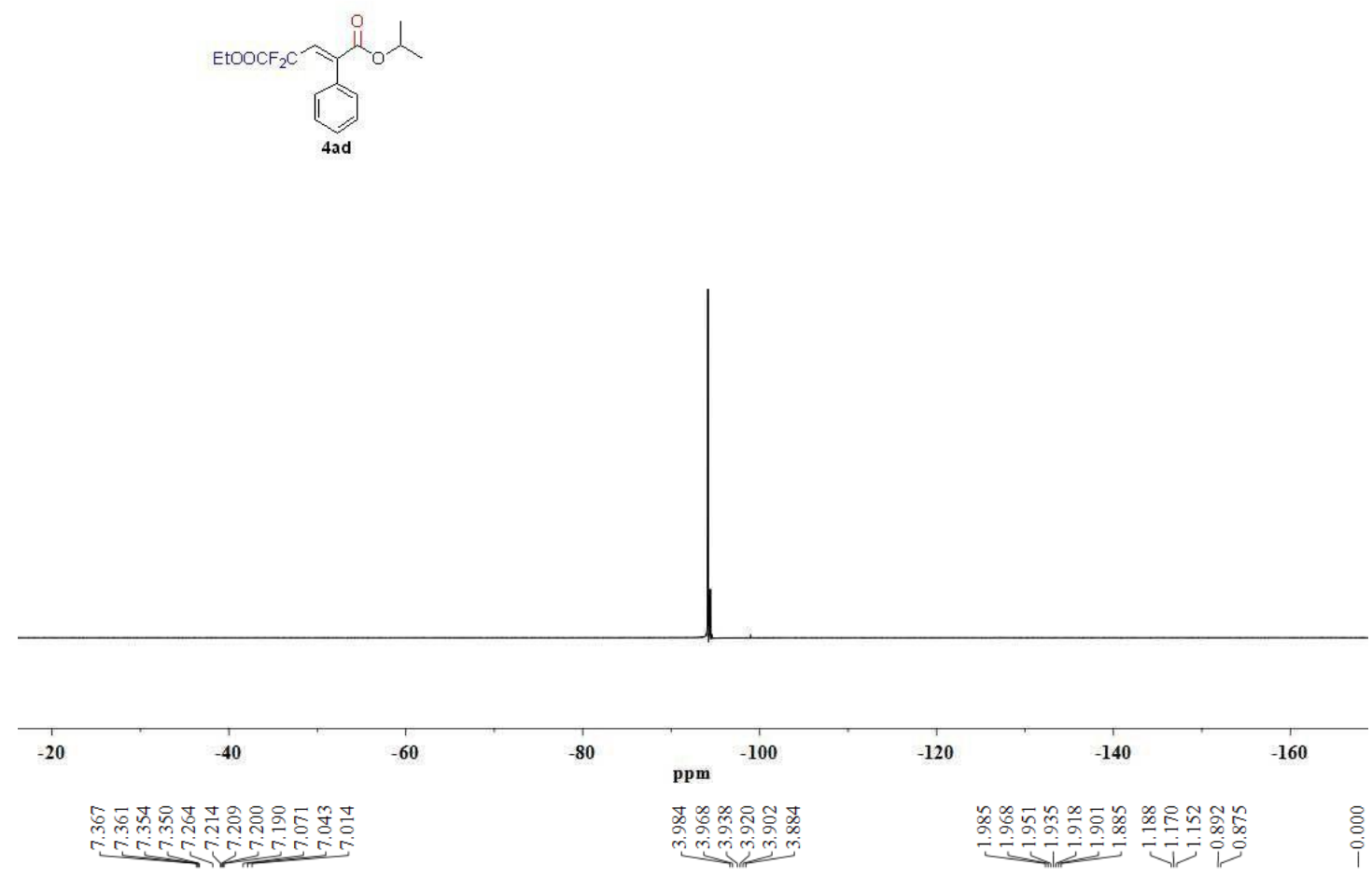

$\mathrm{EtOOCF}_{2} \mathrm{C}$

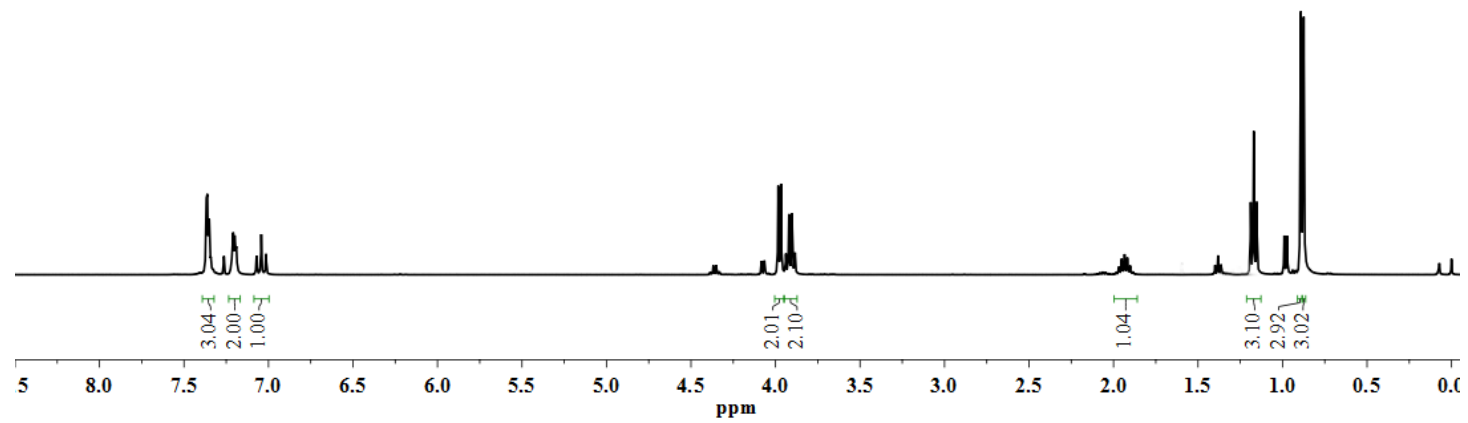




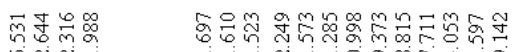

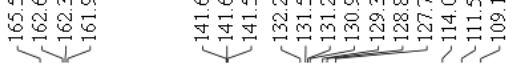

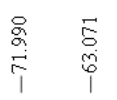

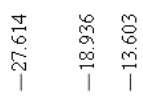

(100CF 2 C

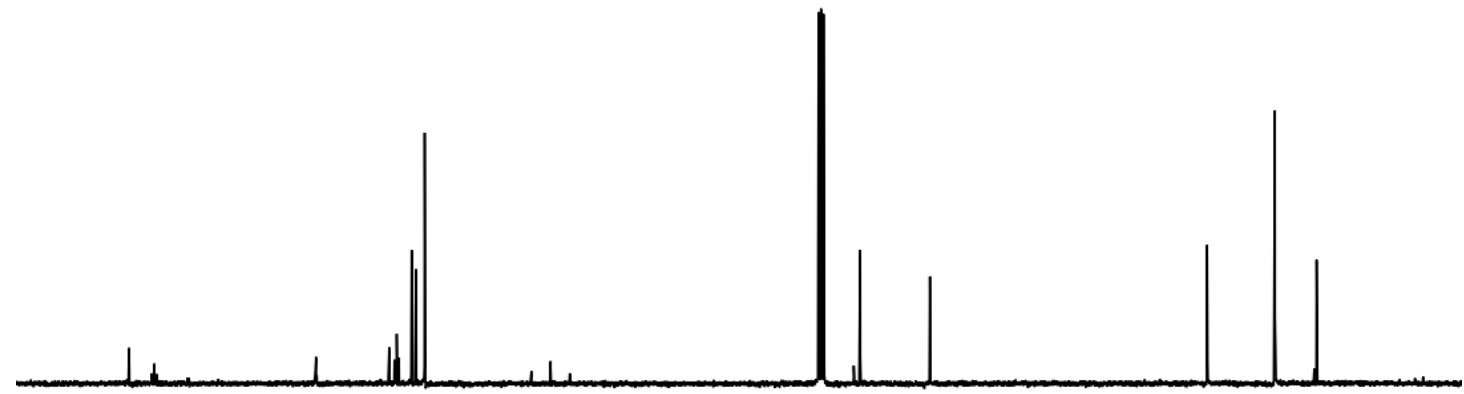

$160 \quad 140$

$120 \quad 100$

$100 \quad$ ppm 8

so 60

a<smiles>CCOC(=O)C=CC(=CC(=O)OCC)C(=O)OCC(C)C</smiles>

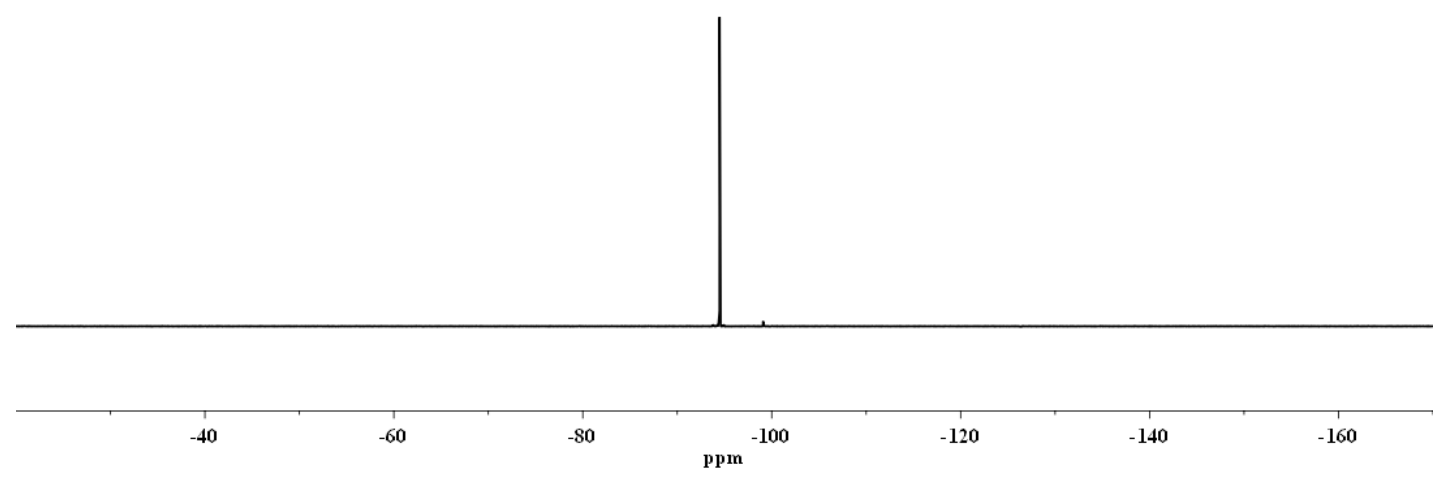


<smiles>CCOC(=O)C(=O)OC1CCCC1</smiles>

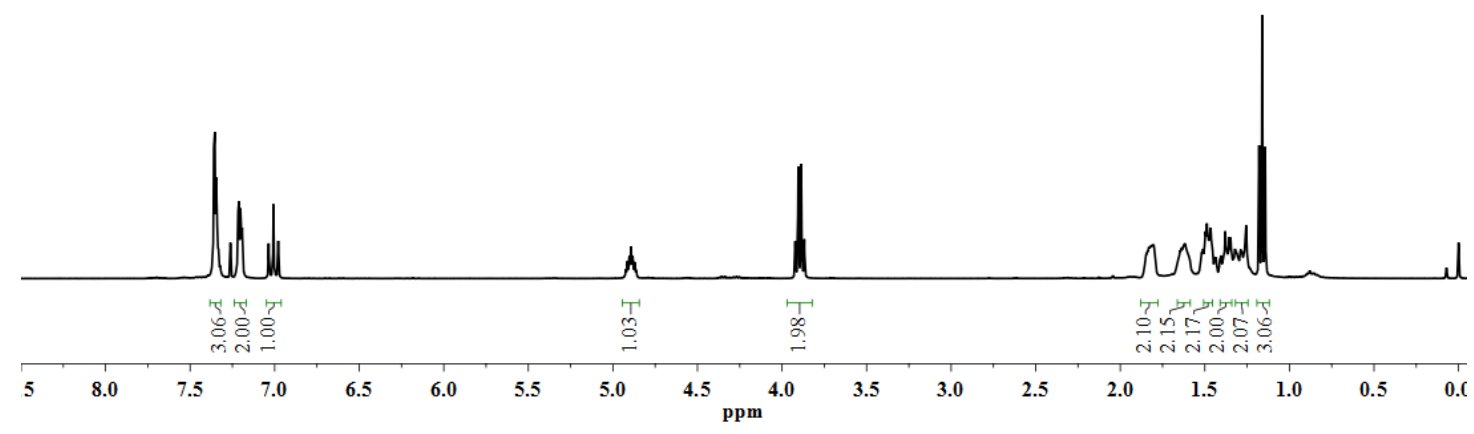

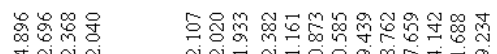

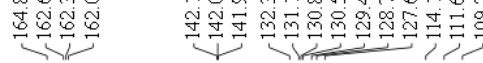
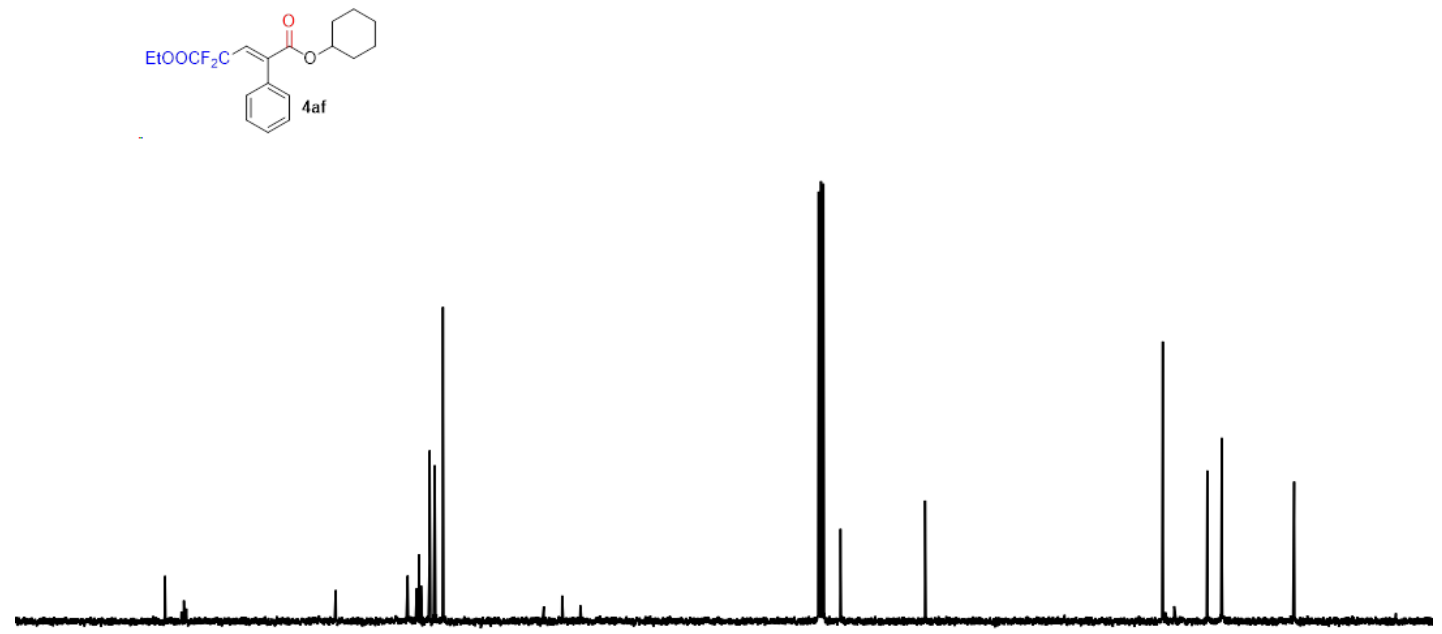

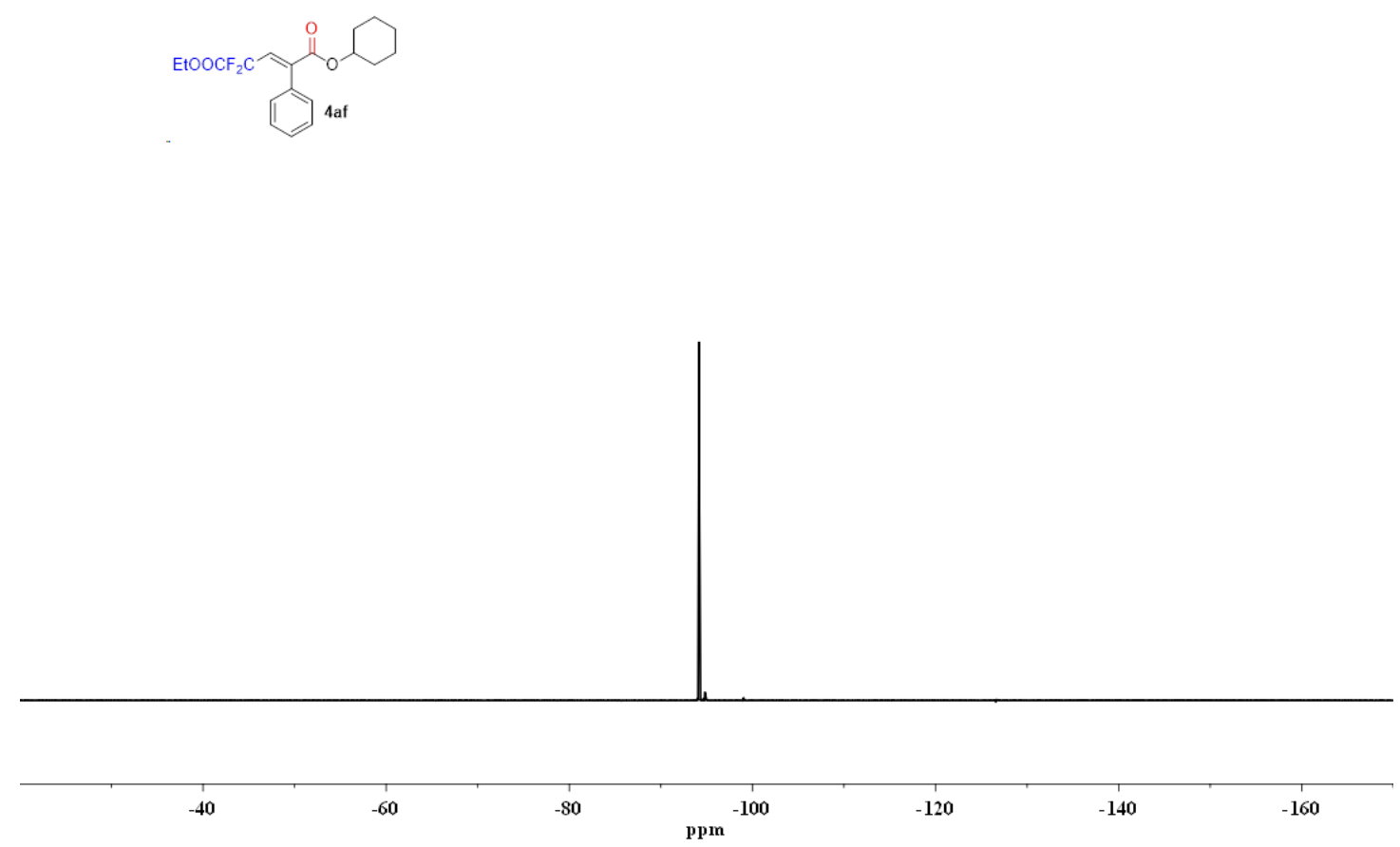

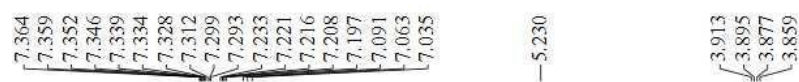

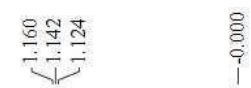<smiles>C=C[c+]1cccc(C(C=CC(=O)OCC)=CC(=O)OCc2ccccc2)cc1</smiles>

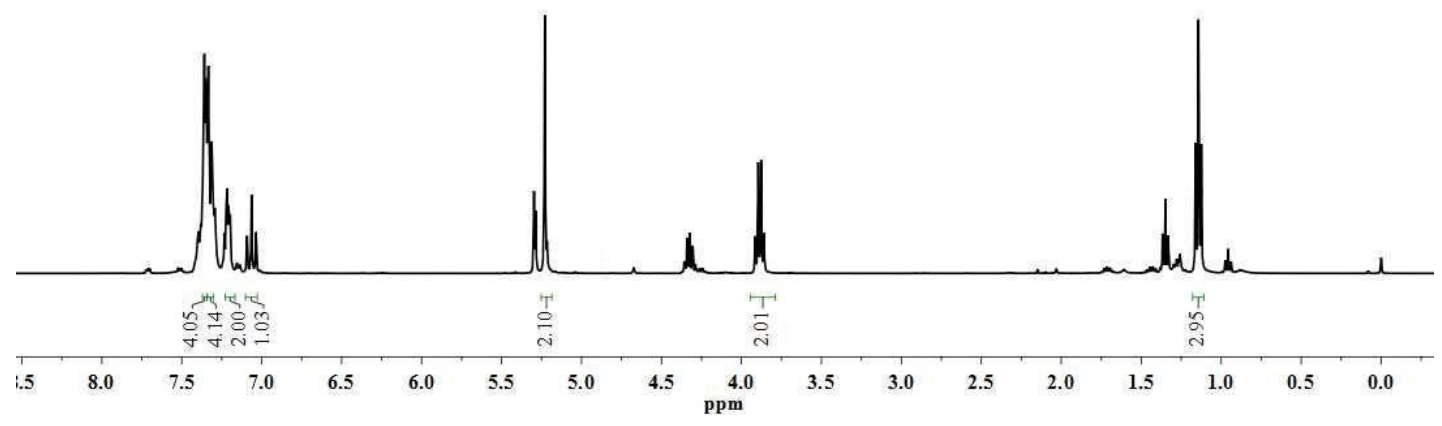



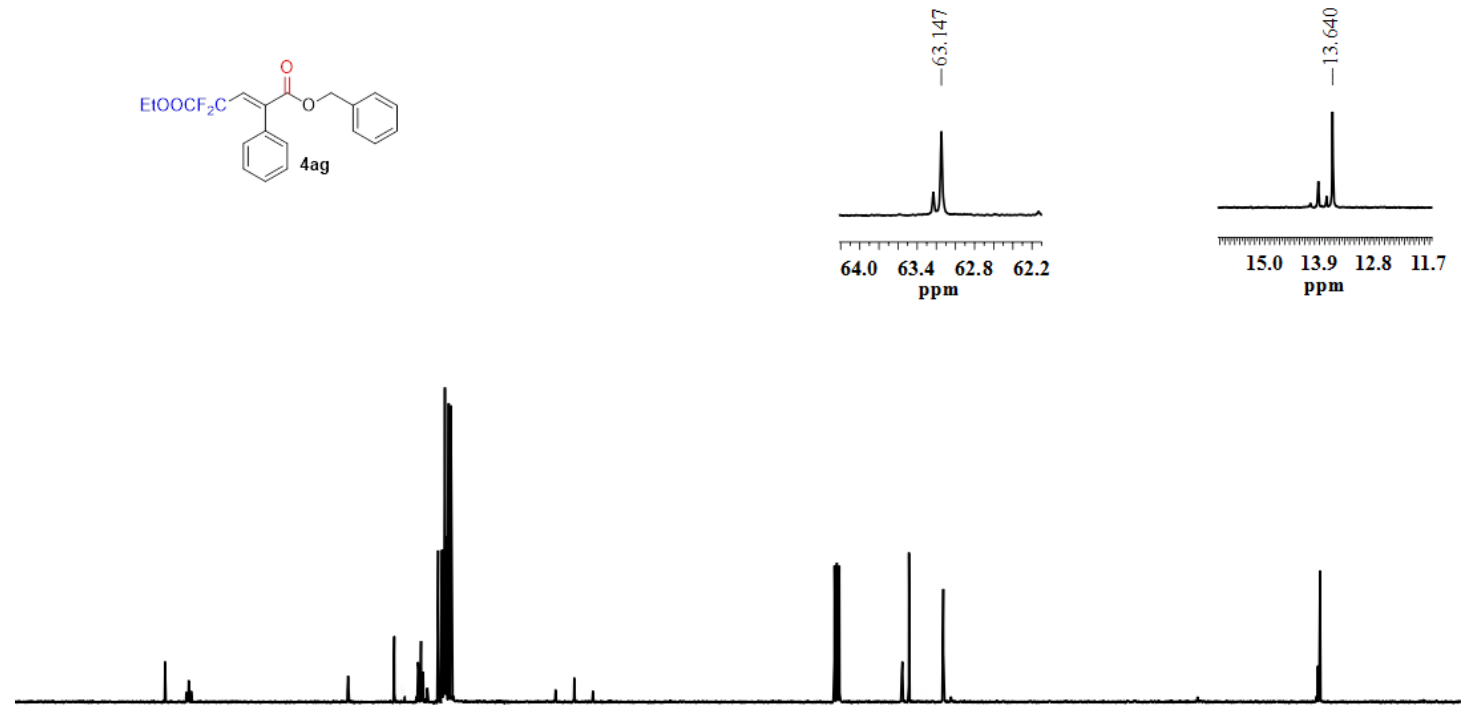

180

$160 \quad 140$

120

$100 \quad$ ppm

80

60

40

20

辛

$\mathrm{EtOOCF}_{2} \mathrm{C}$

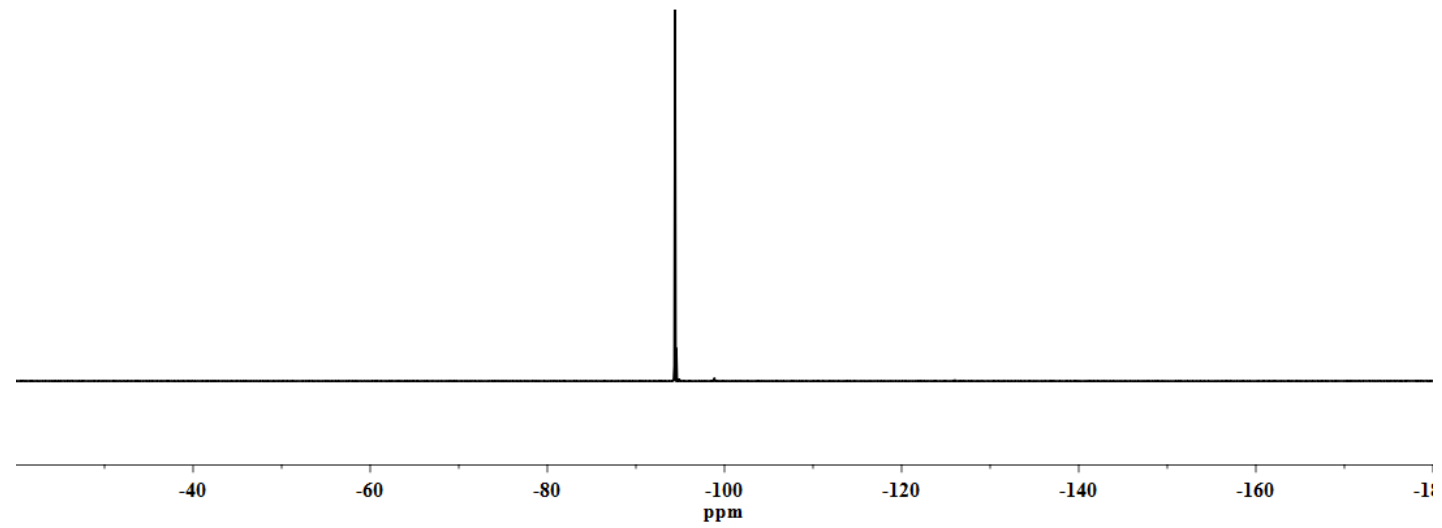


<smiles>CCOC(=O)C=CC(=CC(=O)OCC)c1ccccc1</smiles>

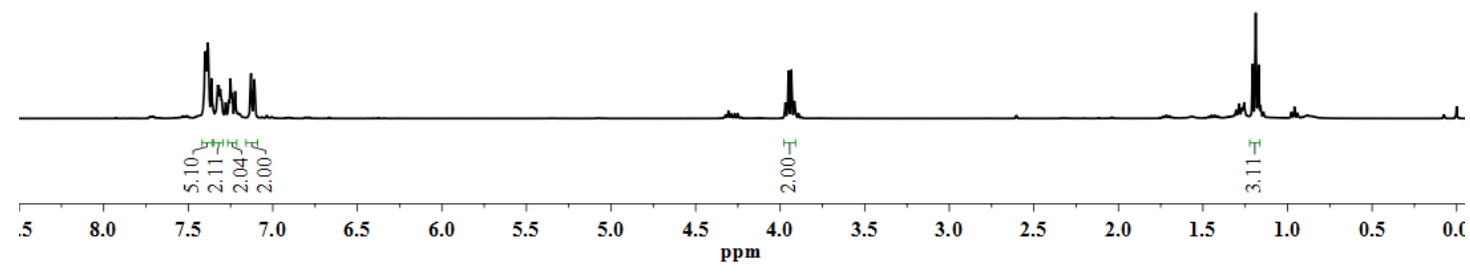

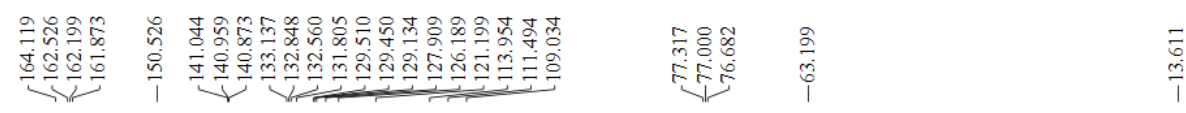

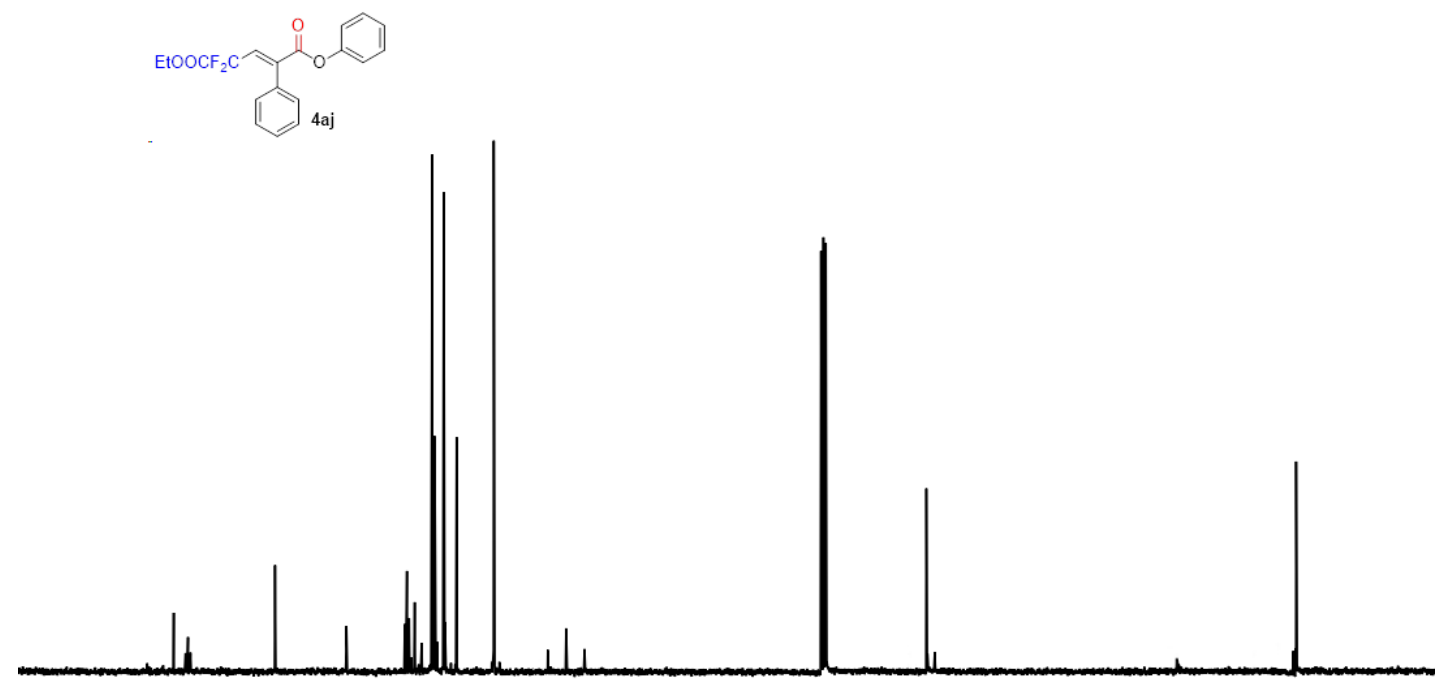

180 160 140 120 100 ppm 80 60 40 


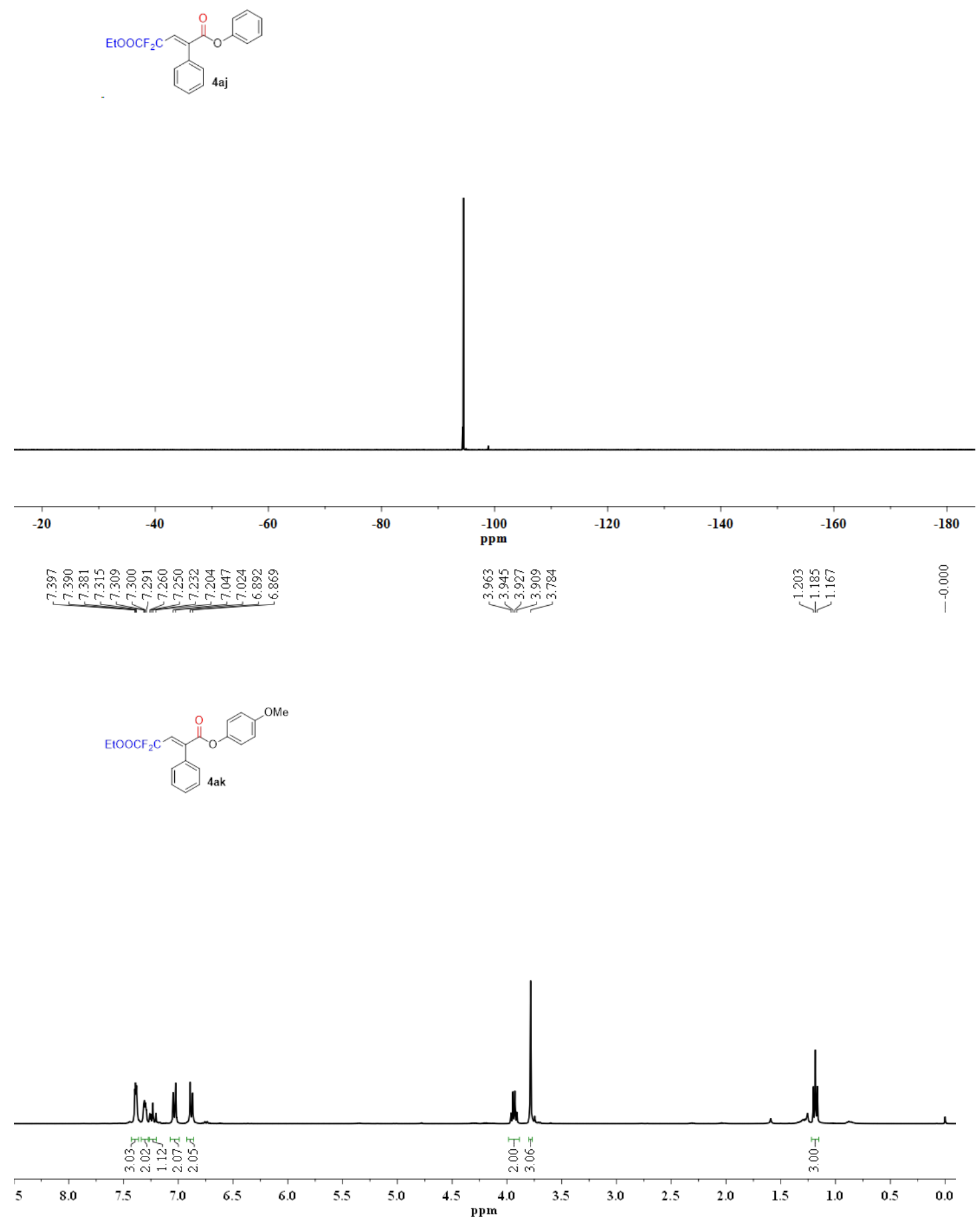




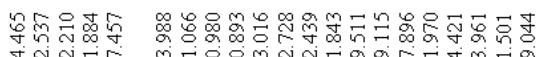

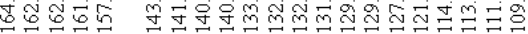

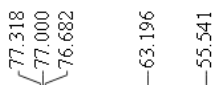

$\stackrel{a}{a}$

$\mathrm{ElOOCF}_{2} \mathrm{C}$

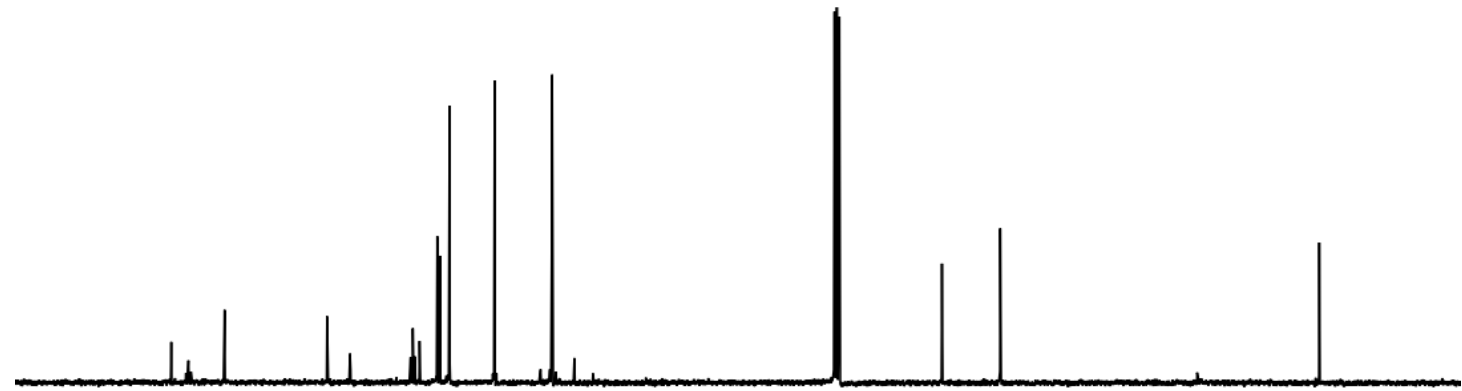

180

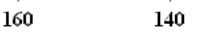

120

$100 \quad$ ppm

so 60

诗

EtOOCF 2

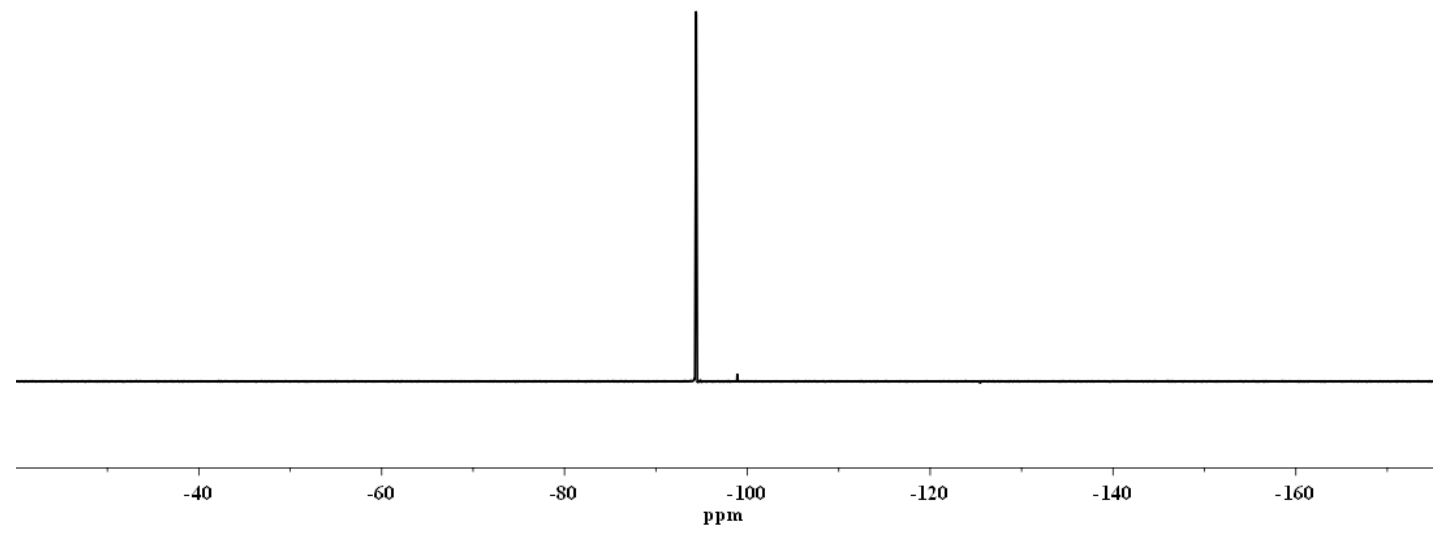




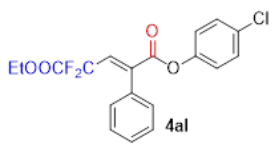

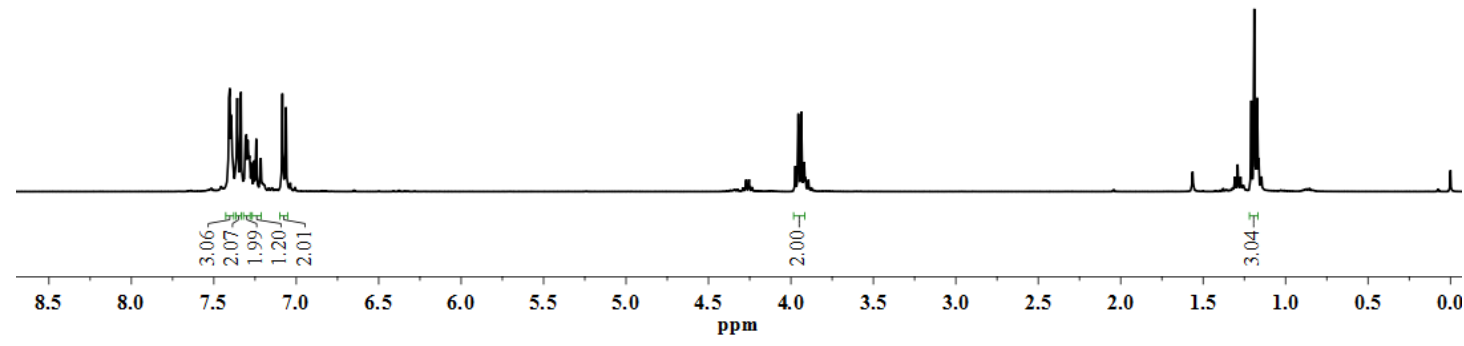

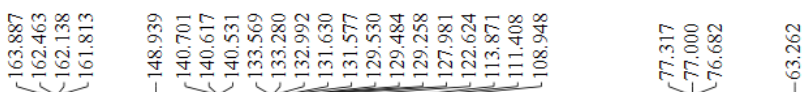
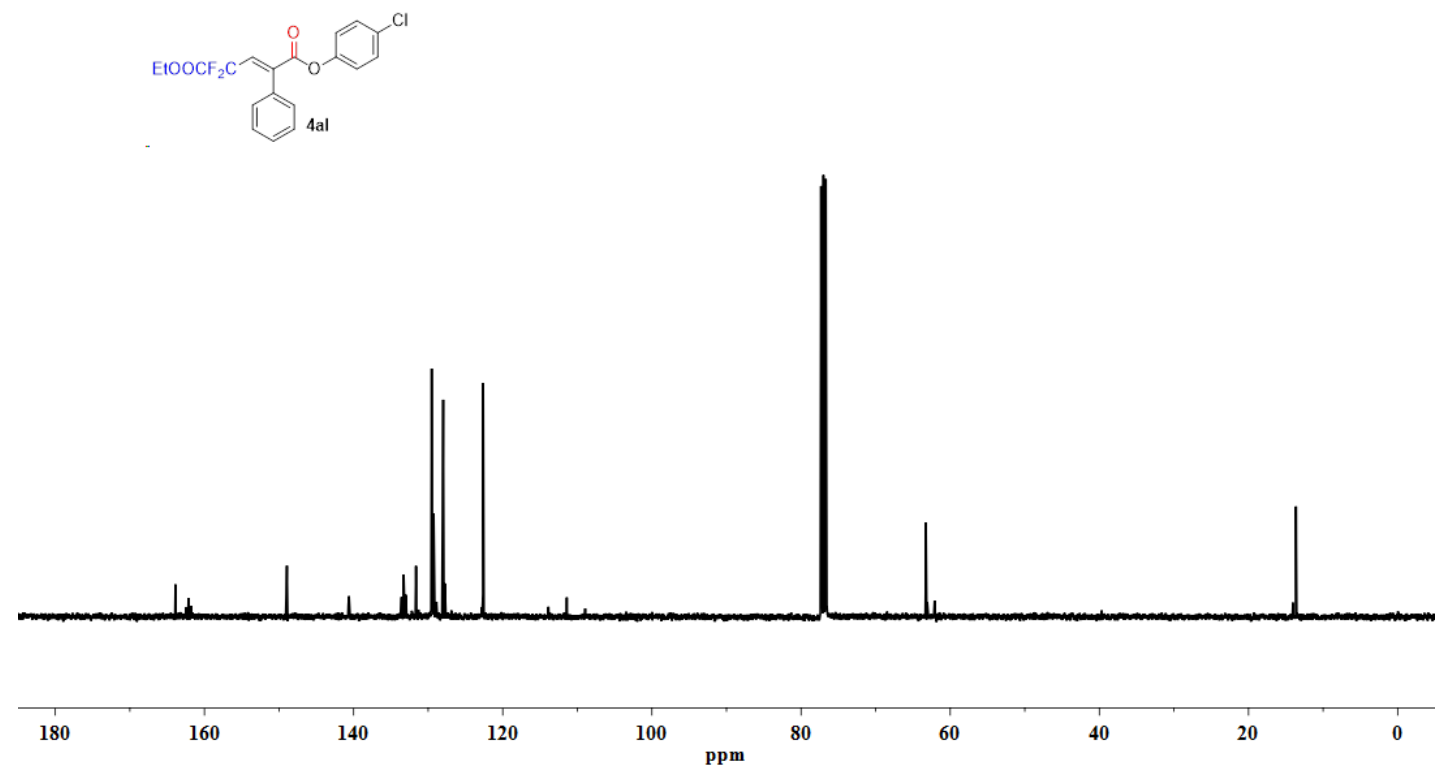

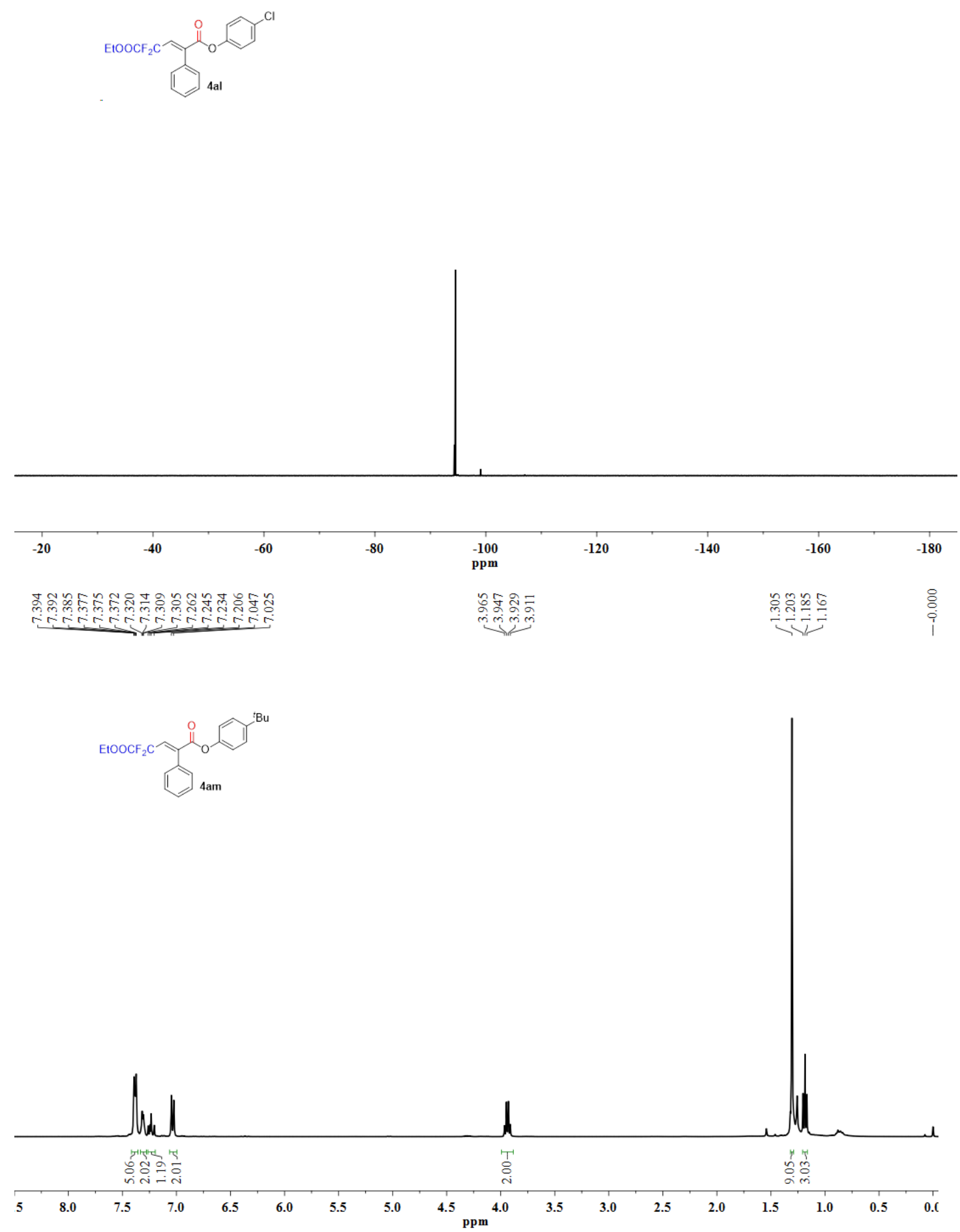

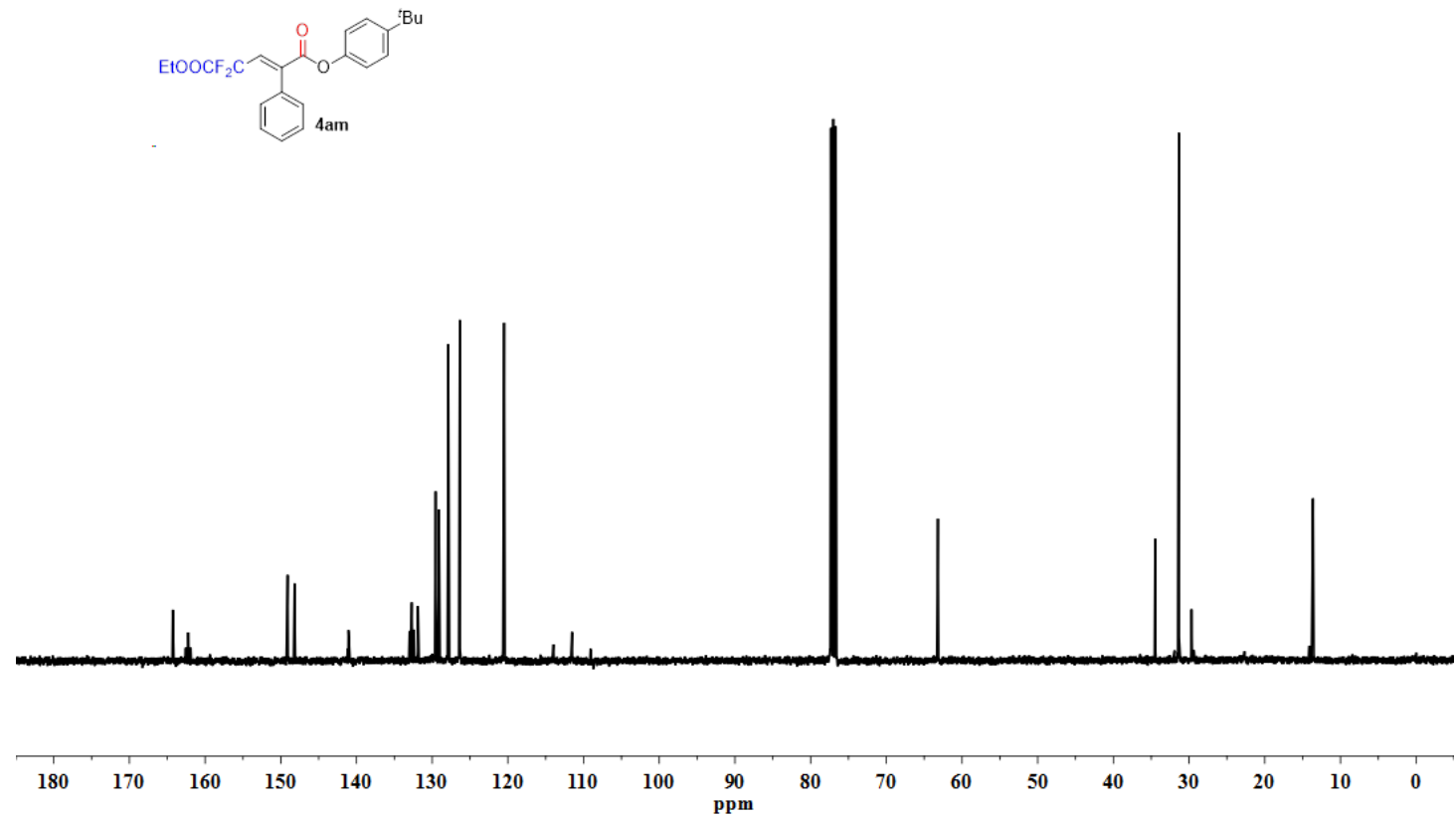

min
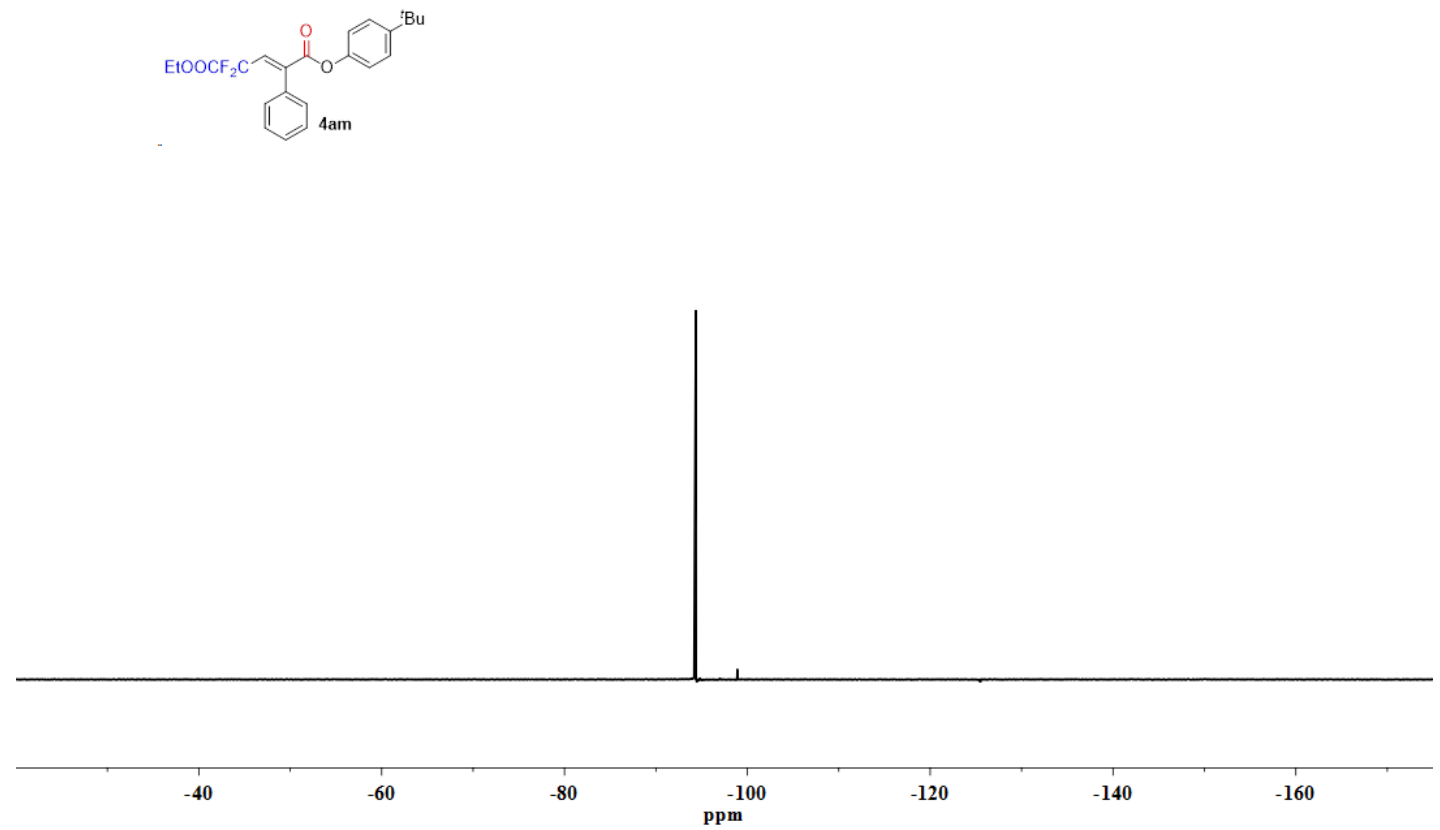


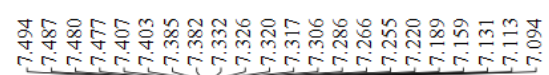<smiles>CCC(NC(=O)C(CC)c1ccccc1)c1ccccc1</smiles>

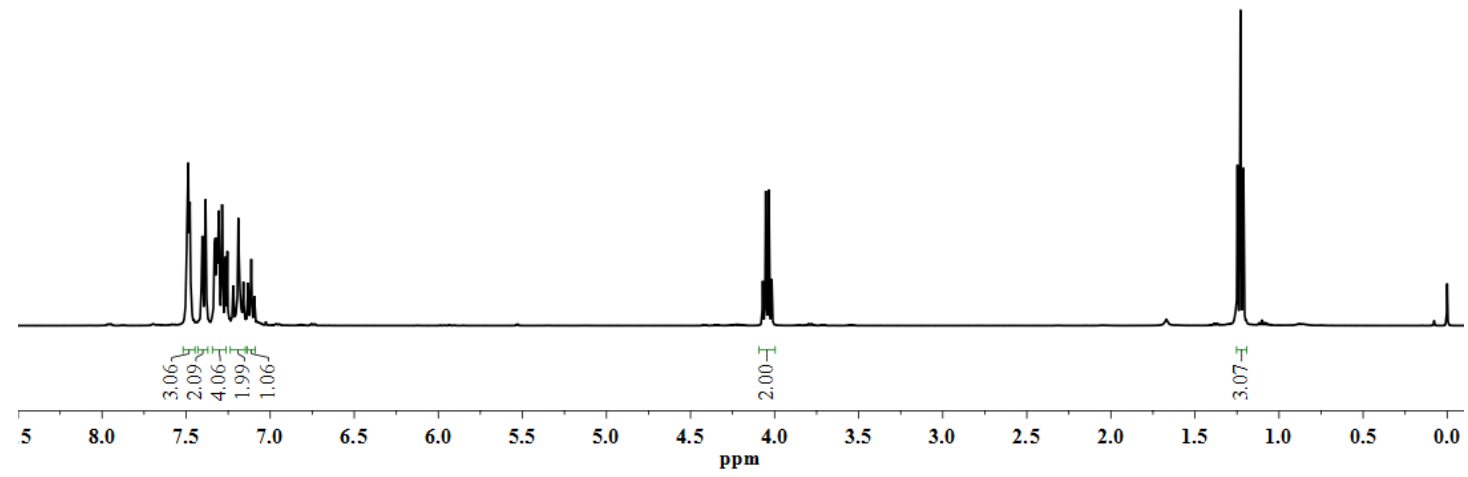

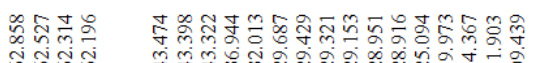

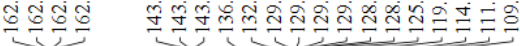

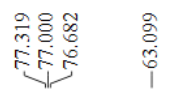

$\frac{8}{\stackrel{8}{0}}$<smiles>CCN(C(=O)Cc1ccccc1)c1ccccc1</smiles>

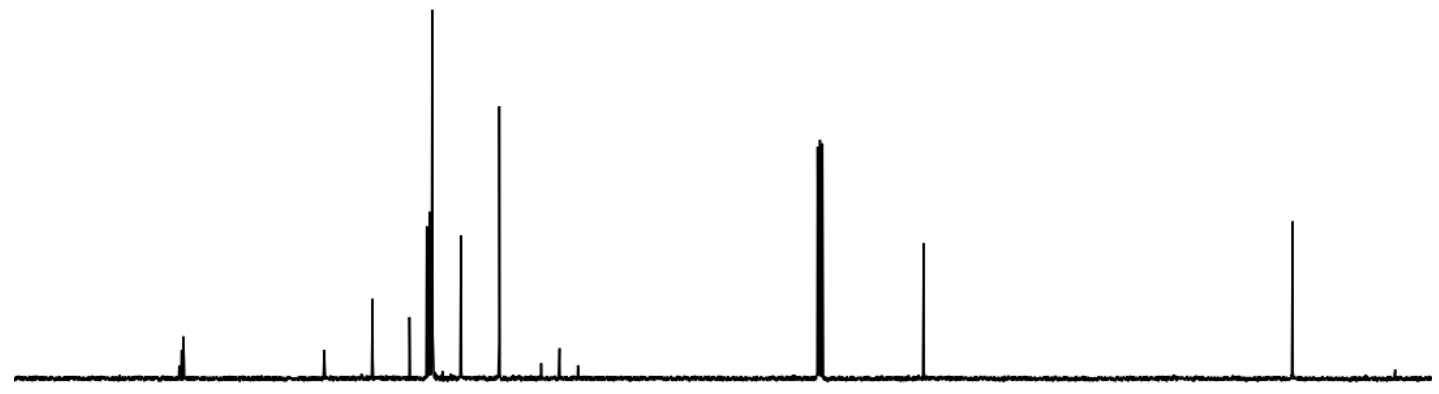

180

160

140

120

100

ppm

80

60

40

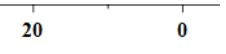


$\mathrm{E}_{10 O \mathrm{CF}_{2} \mathrm{C}}$
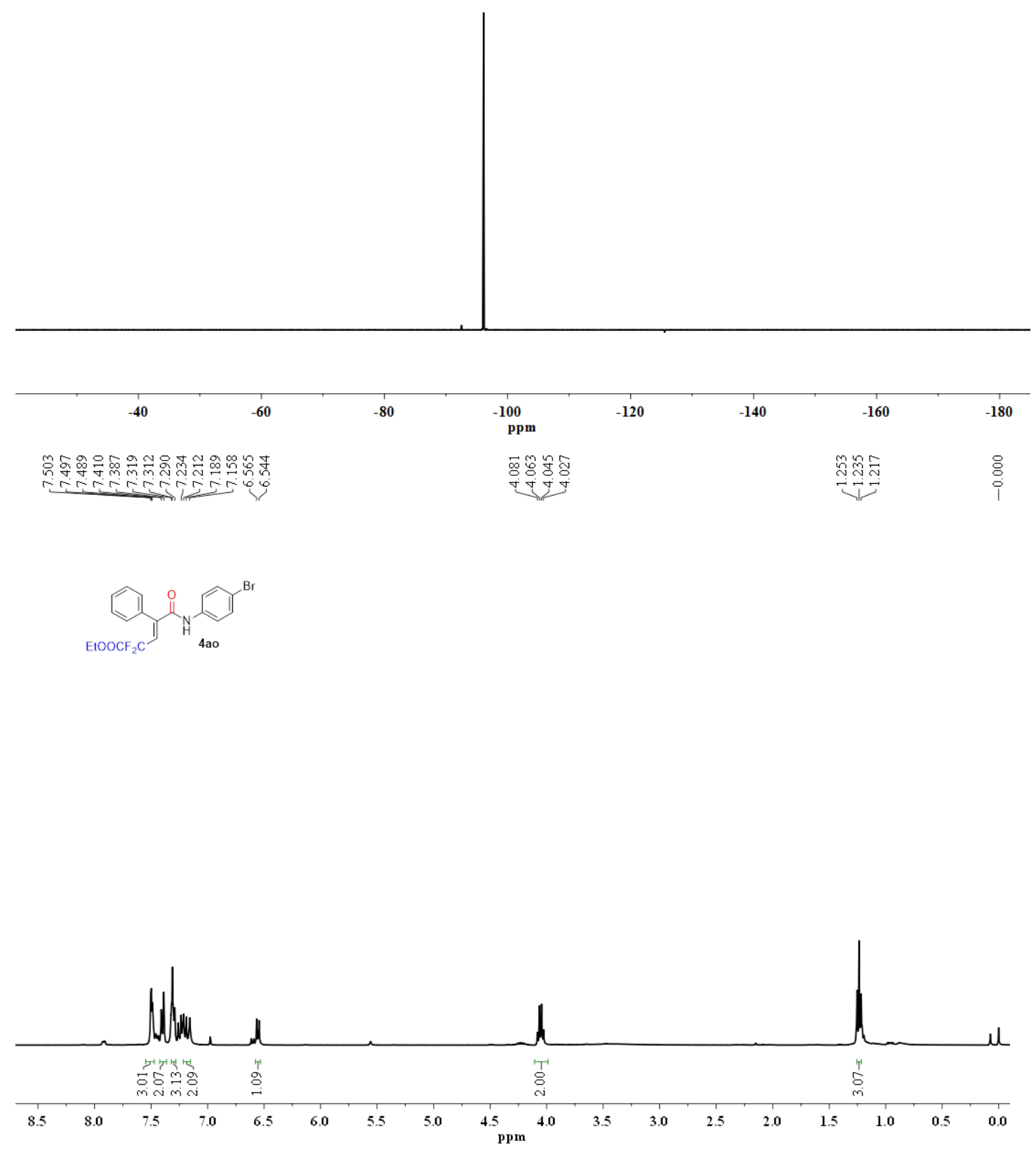


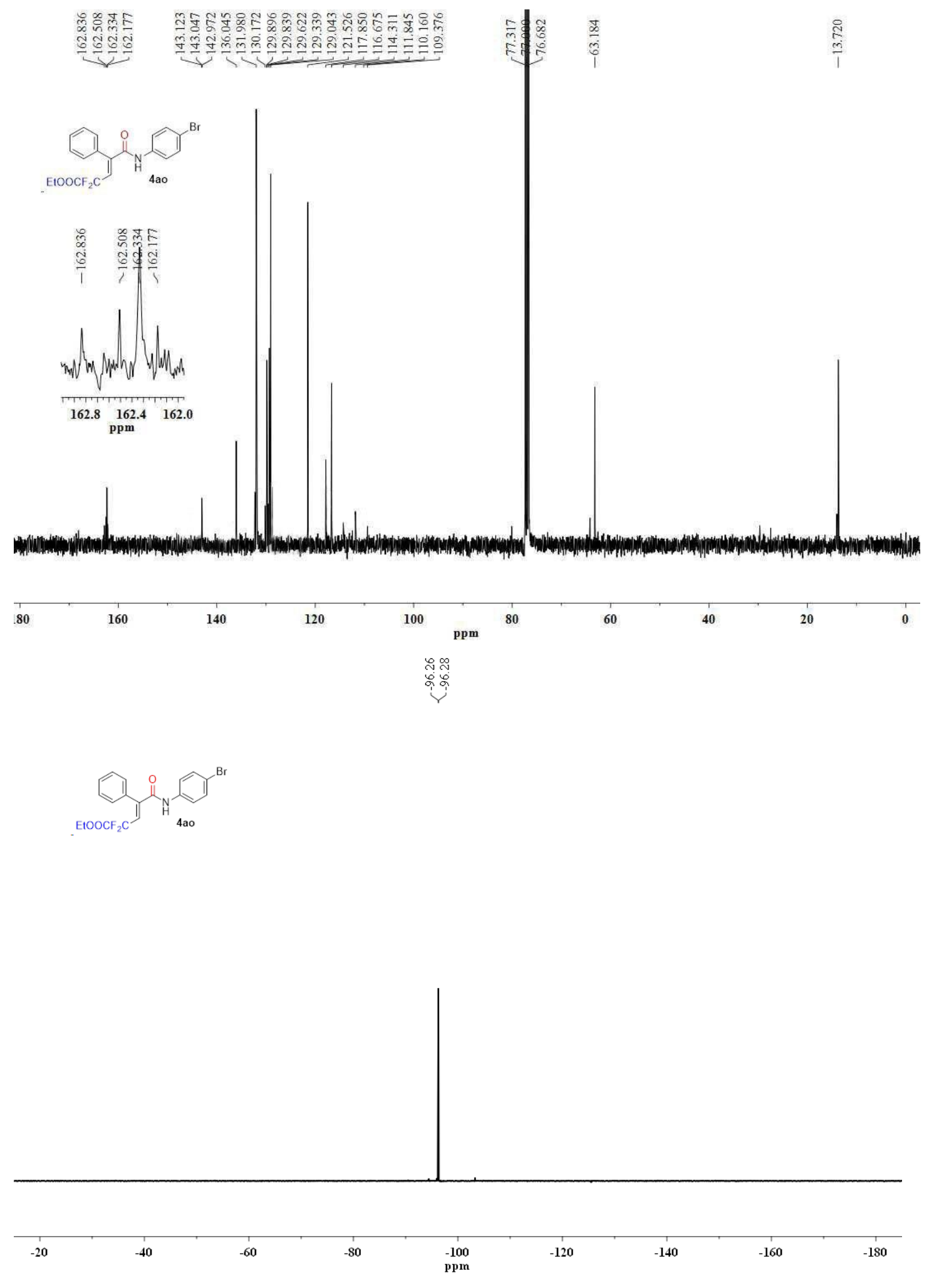



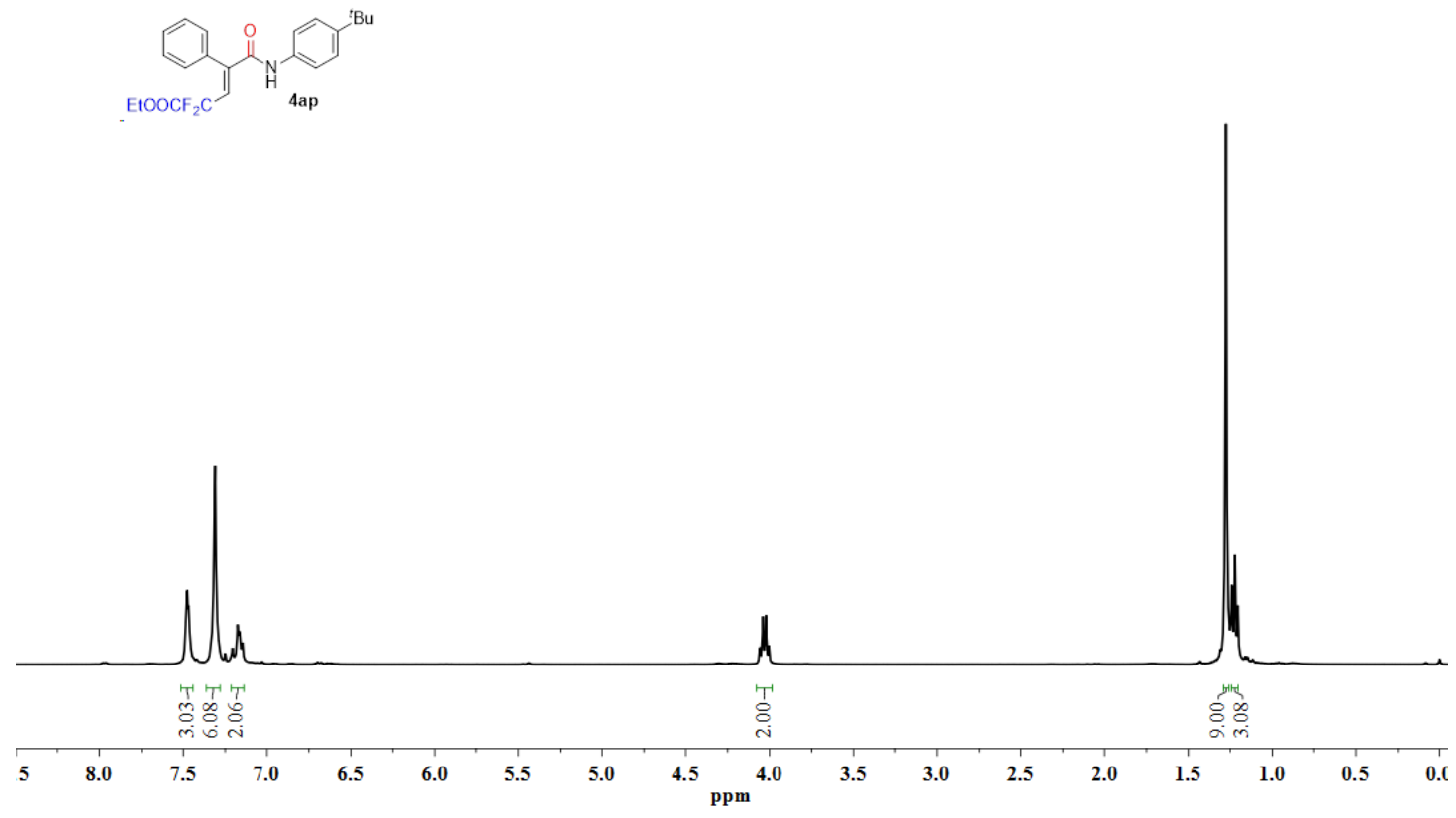

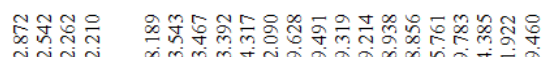

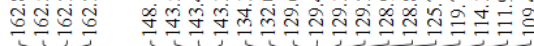
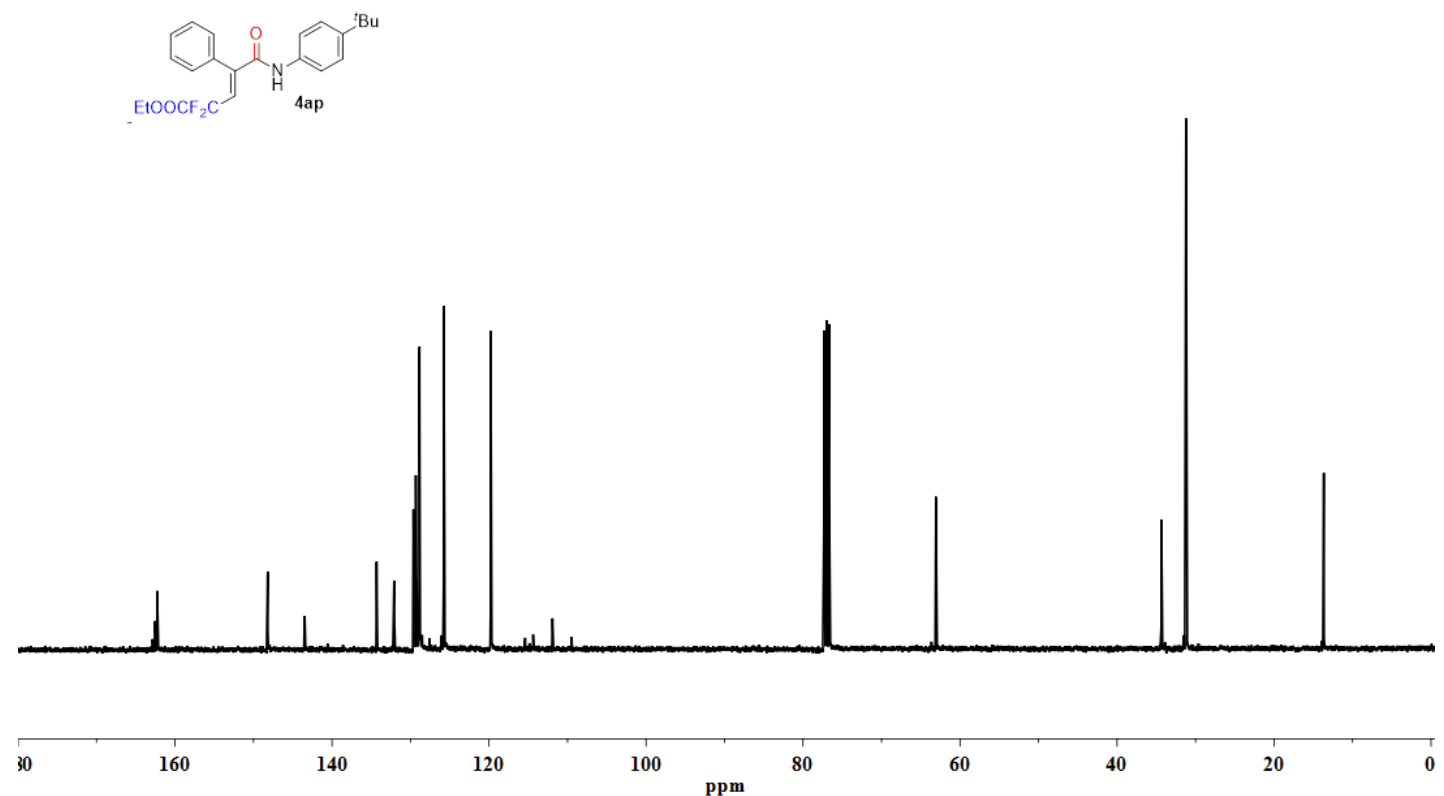
E10OCF $\mathrm{F}_{2} \mathrm{C}$
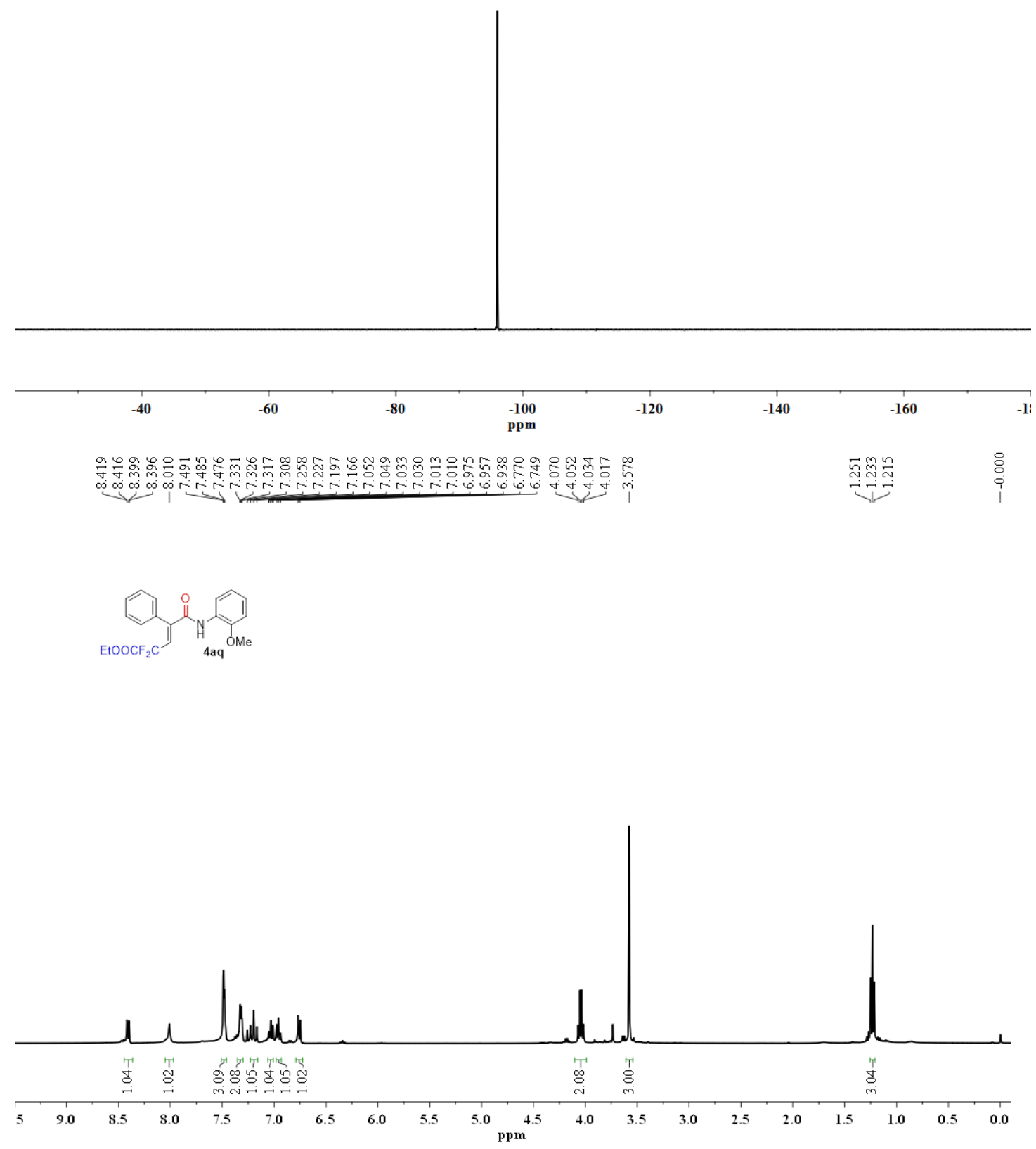


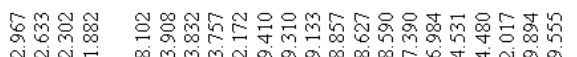

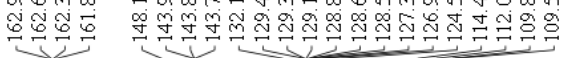

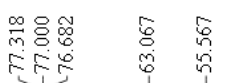

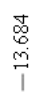

${\text { EroocF } \mathrm{F}_{2} \mathrm{C}}_{4 \mathrm{aq}}$

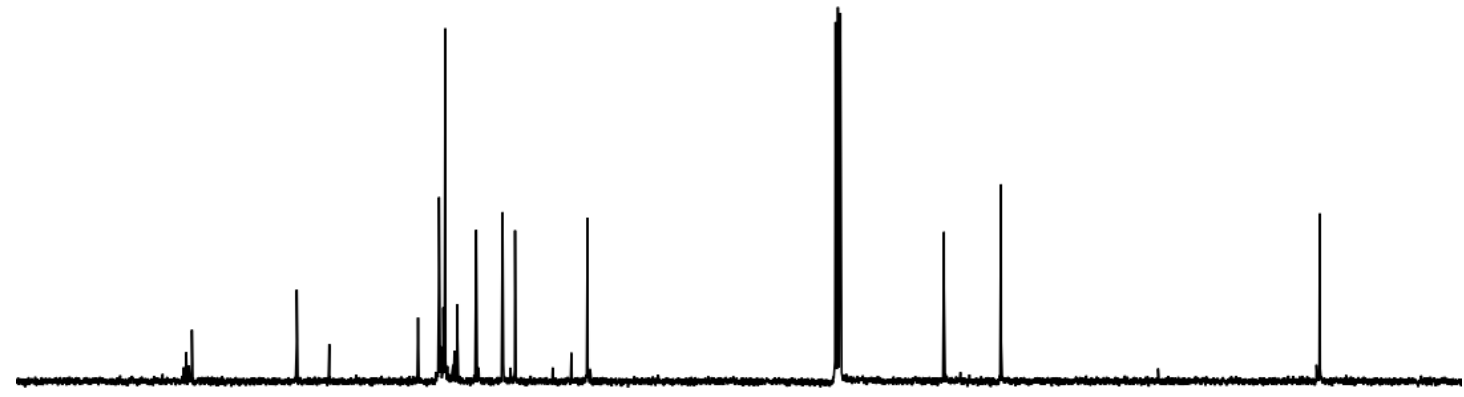

180

$160 \quad 140$

120

$100 \quad$ ppm

$80 \quad 60$

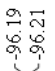

$\mathrm{E}_{100 \mathrm{CF}} \mathrm{C}$

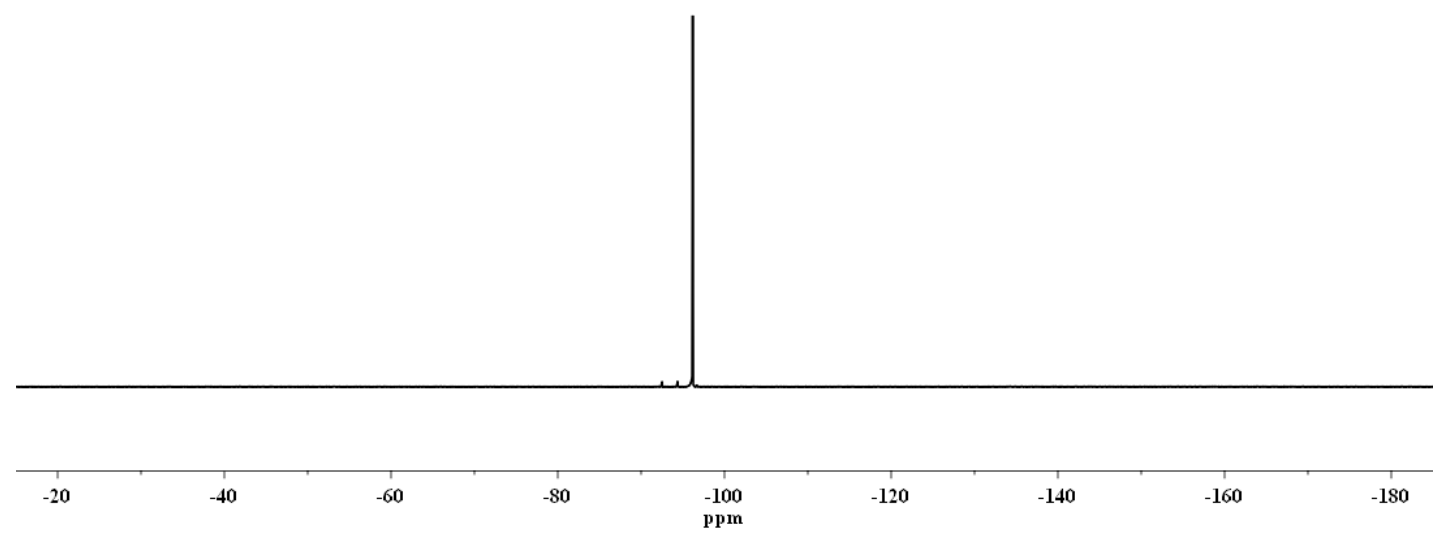



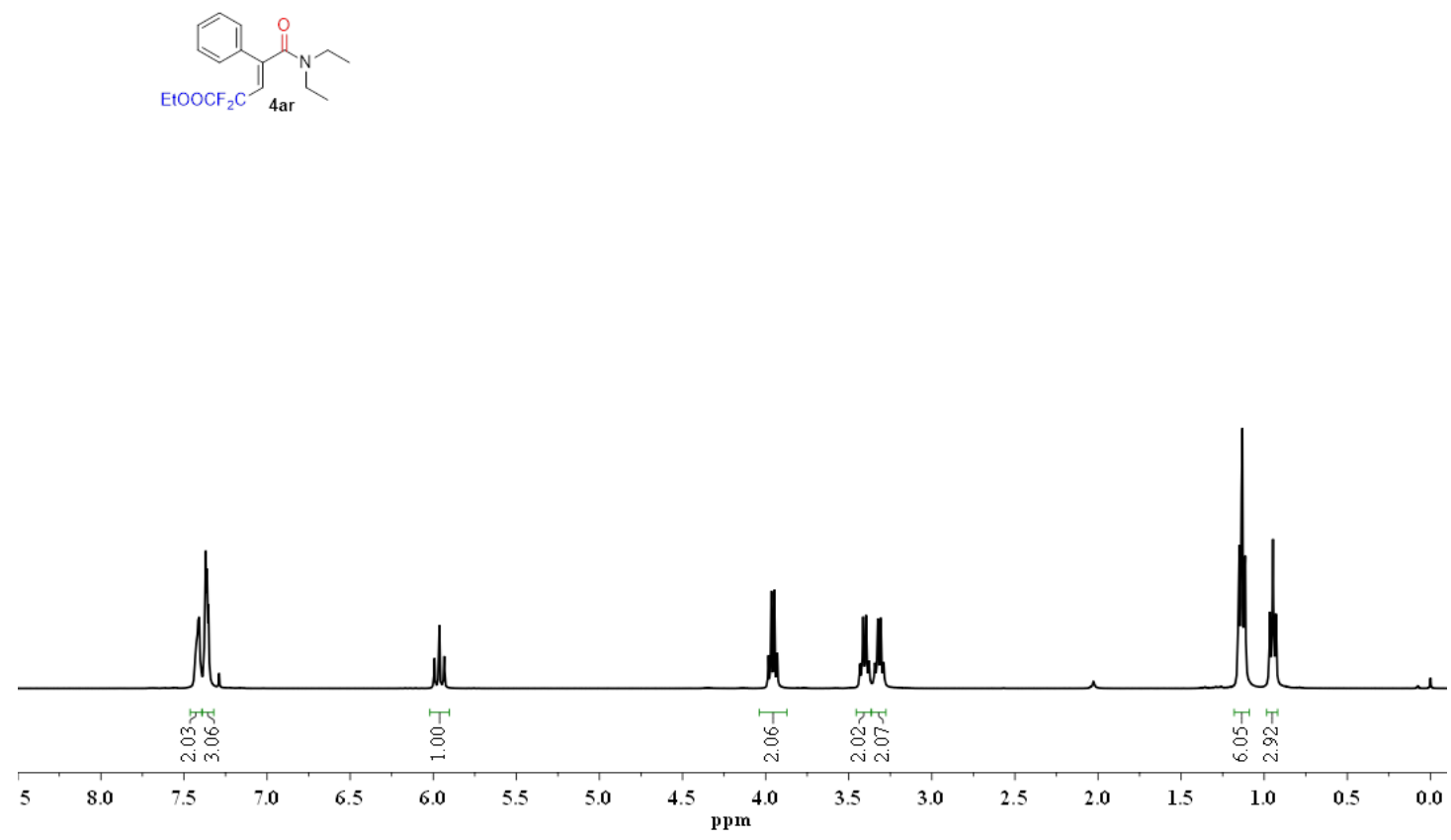

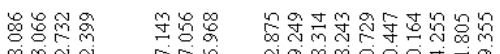

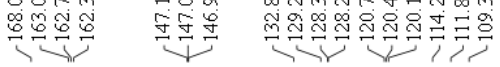

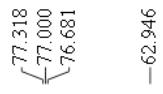

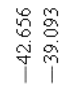

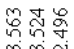

$\sqrt[m]{\mathrm{m} i}$
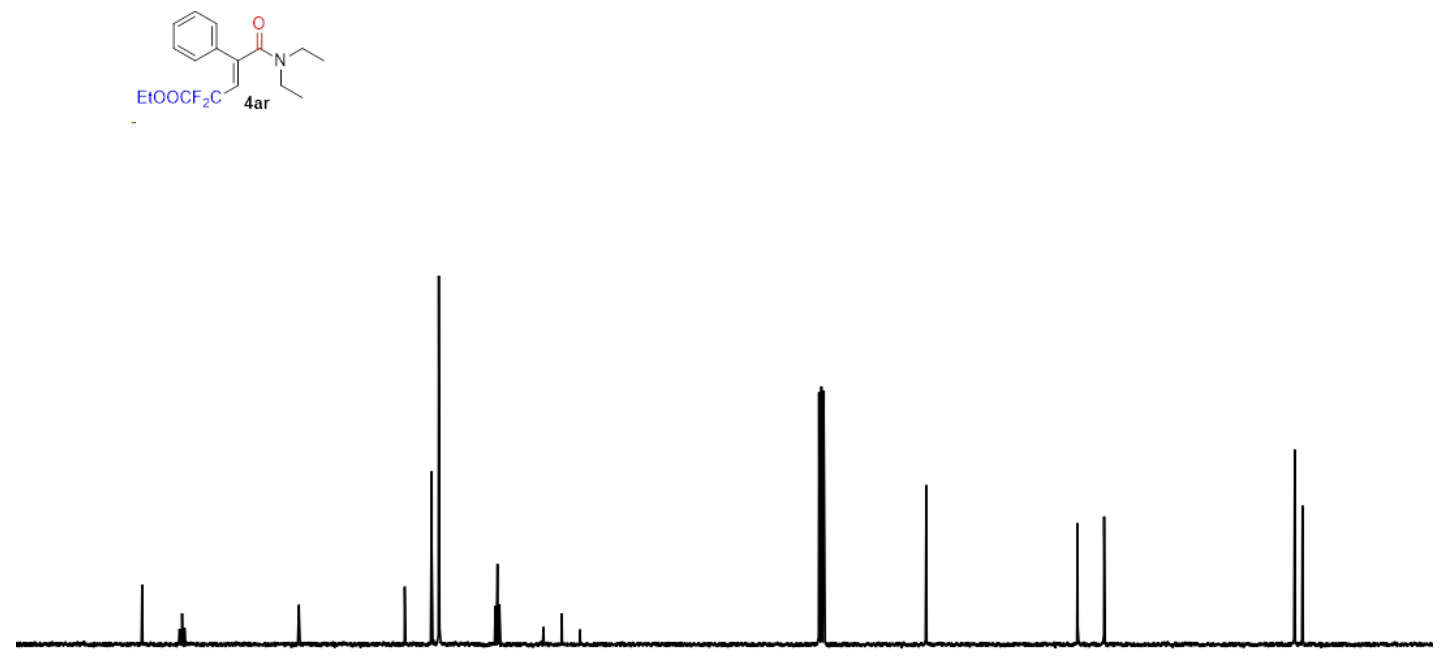

180

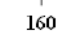

140

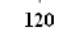

10

ppm

so

60

40

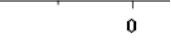


<smiles>CCN(CC)C(=O)C(=CC=O)c1ccccc1</smiles>
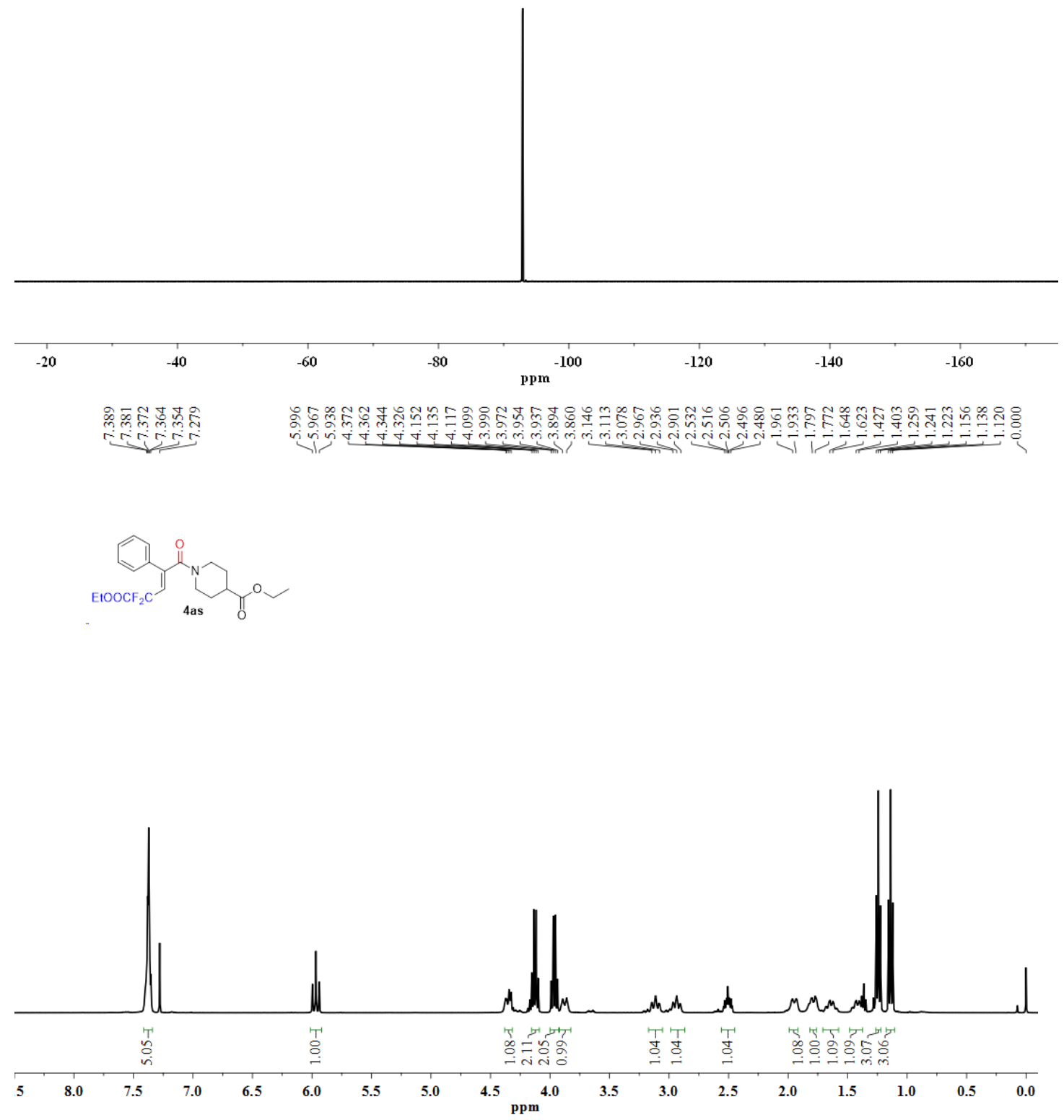


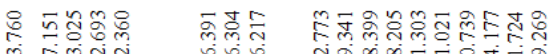

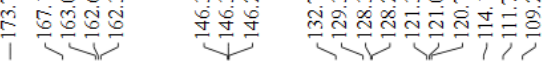

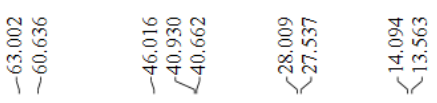
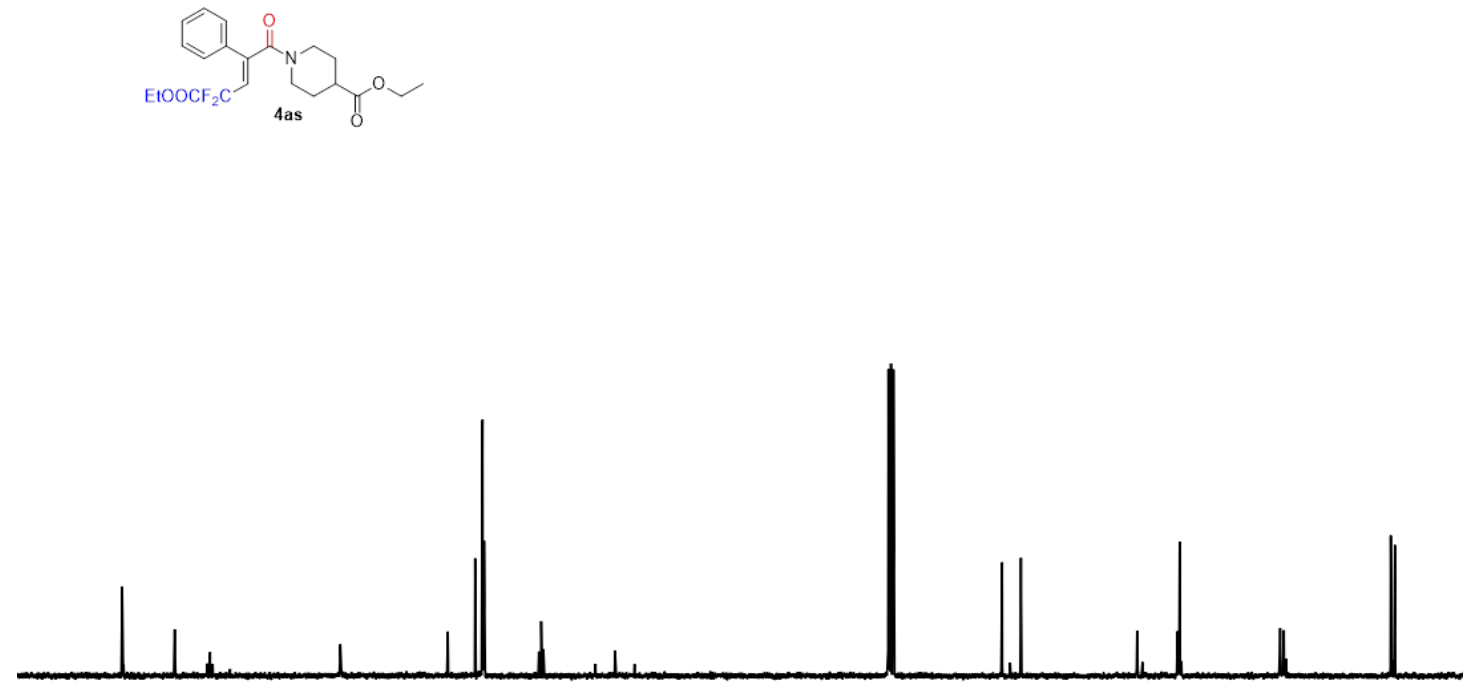

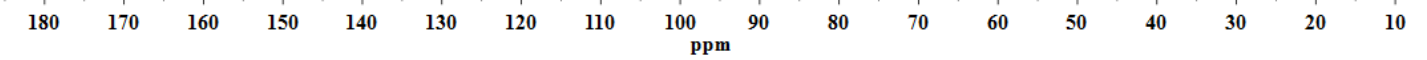
$\stackrel{\bar{i}}{\grave{i}}$
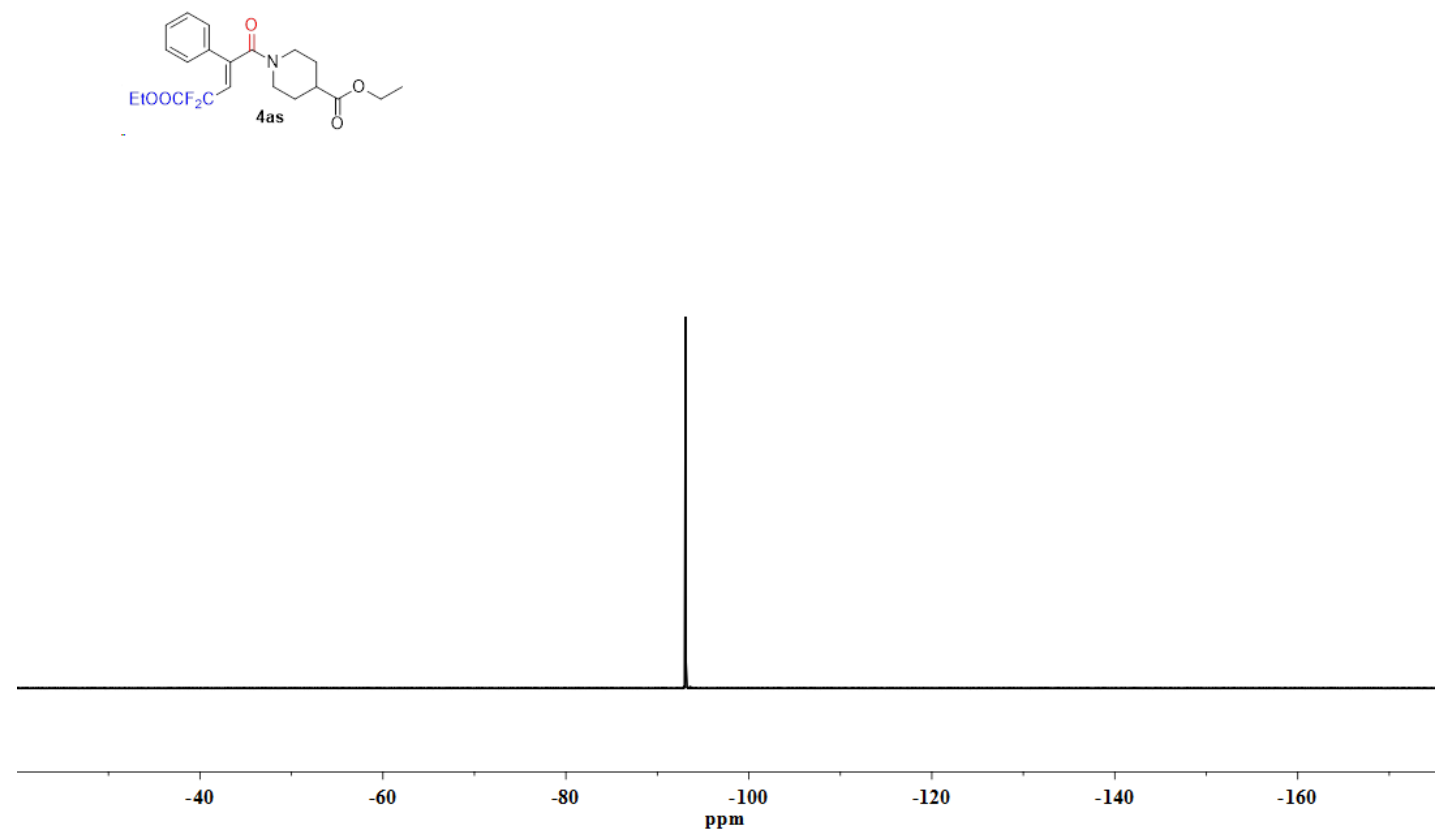
<smiles>O=C(/C=C(\C(=O)N1CCCCC1)c1ccccc1)N1CCCCC1</smiles>

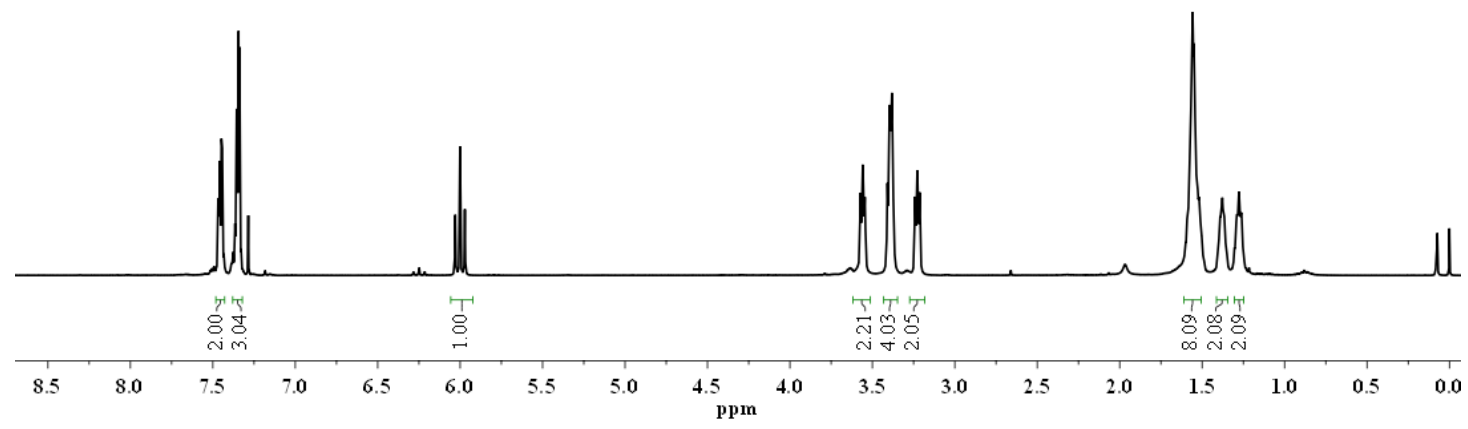

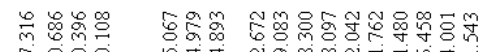

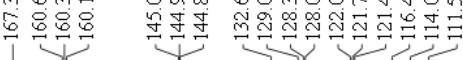

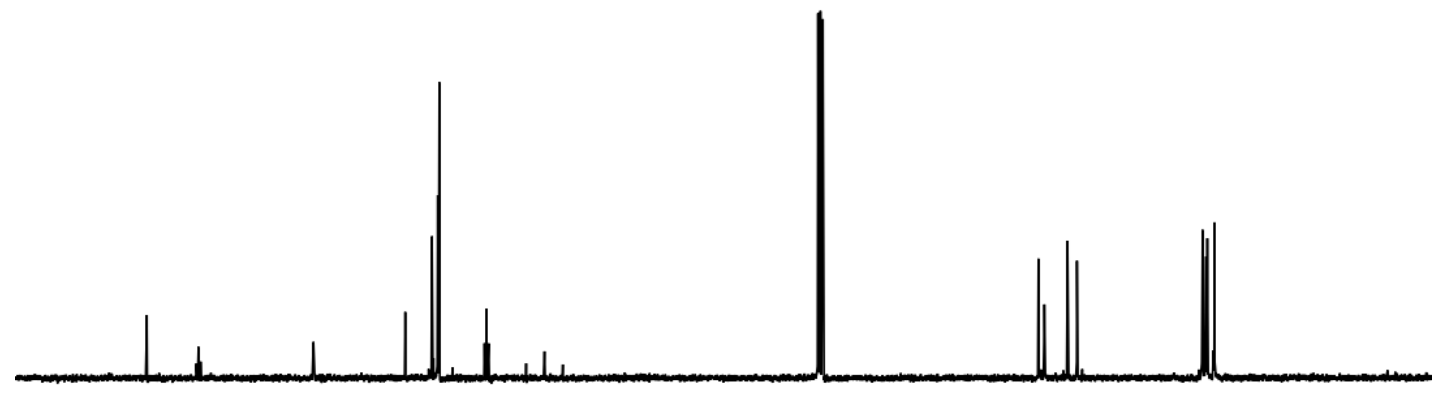


<smiles>O=C(C(=CC=C1C=CC=C1)C(=O)N1CCCCC1)N1CCCCC1</smiles>
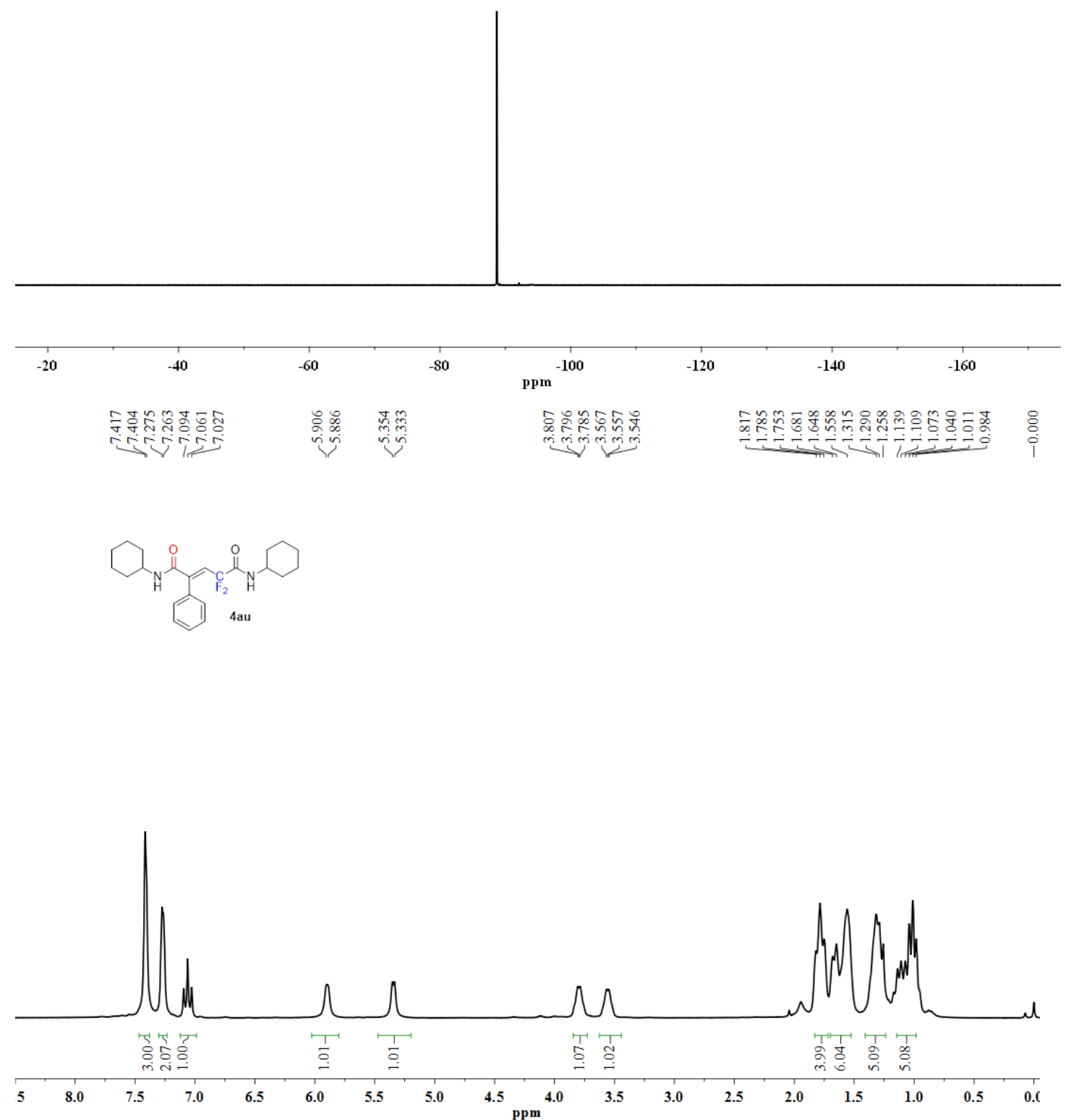


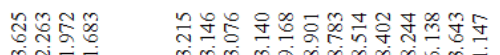

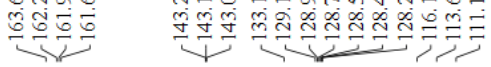

잉훙

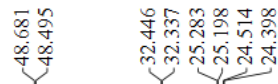

$\mathrm{O}_{4 \text { au }}$

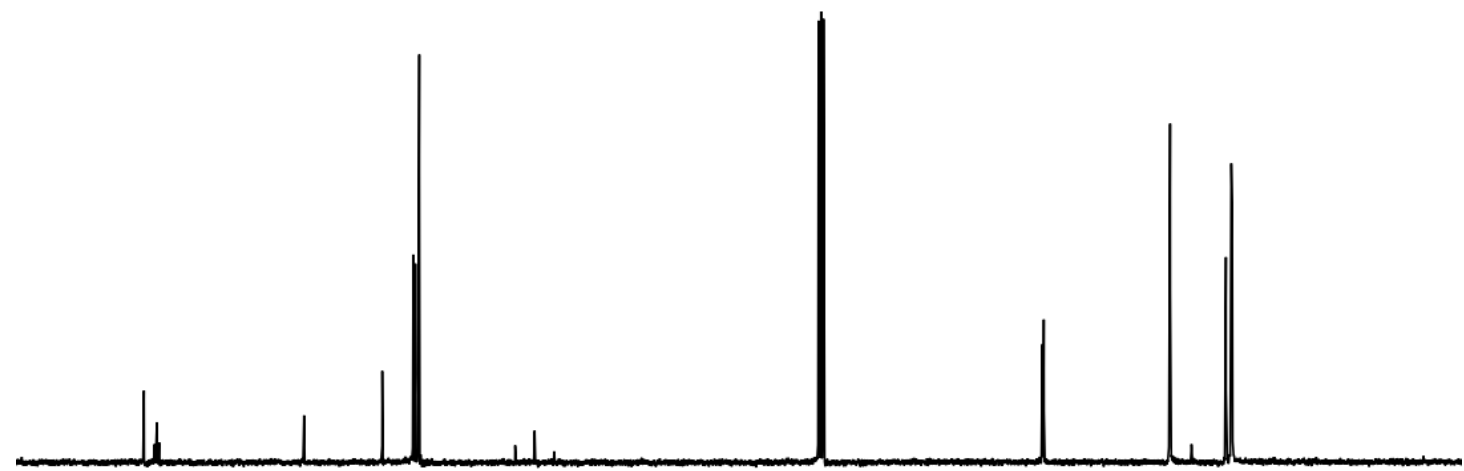

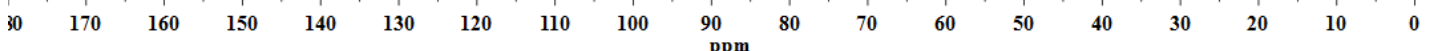

å
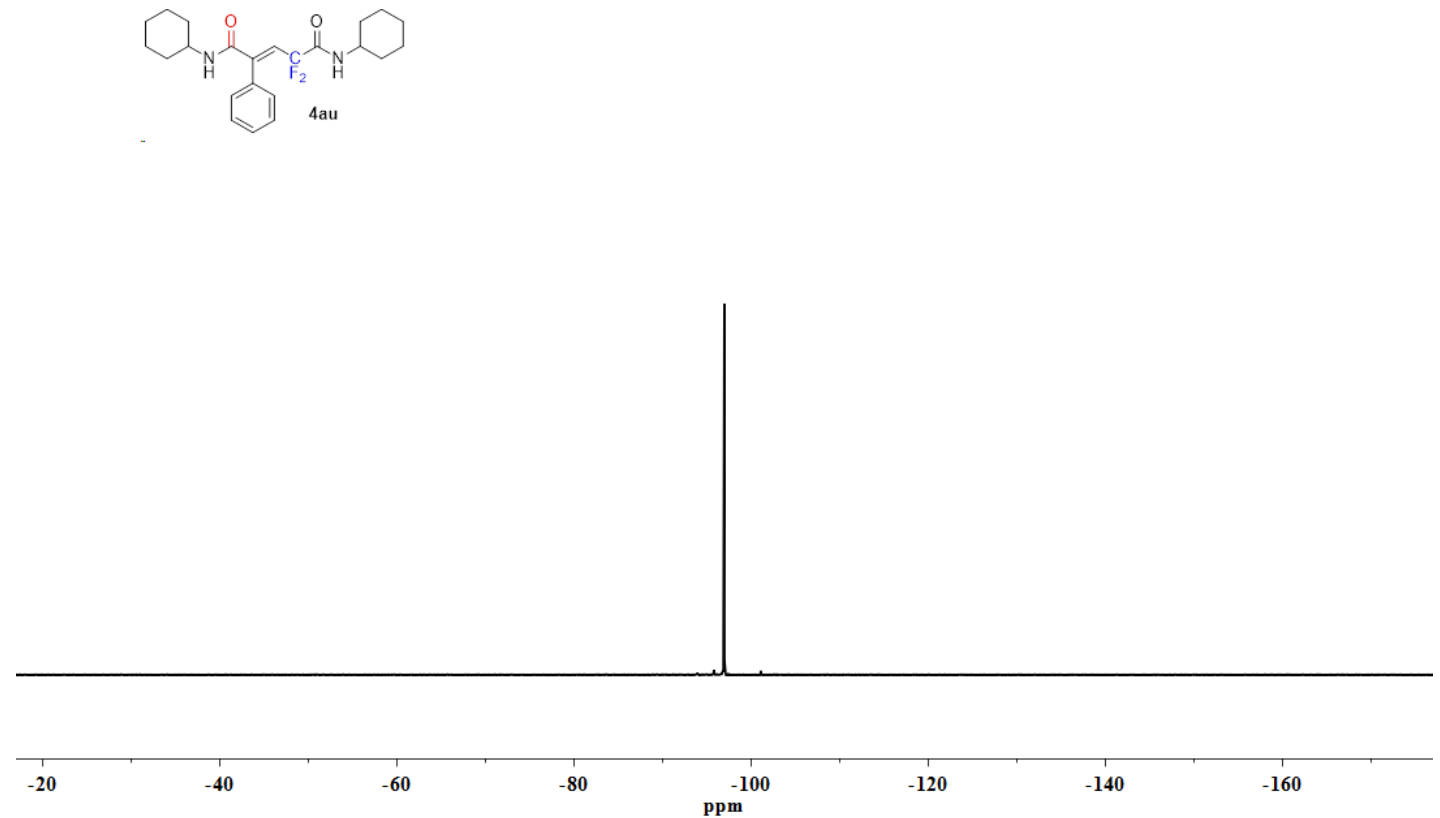
<smiles>CCOC(=O)OCCC(c1ccccc1)c1ccccc1</smiles>

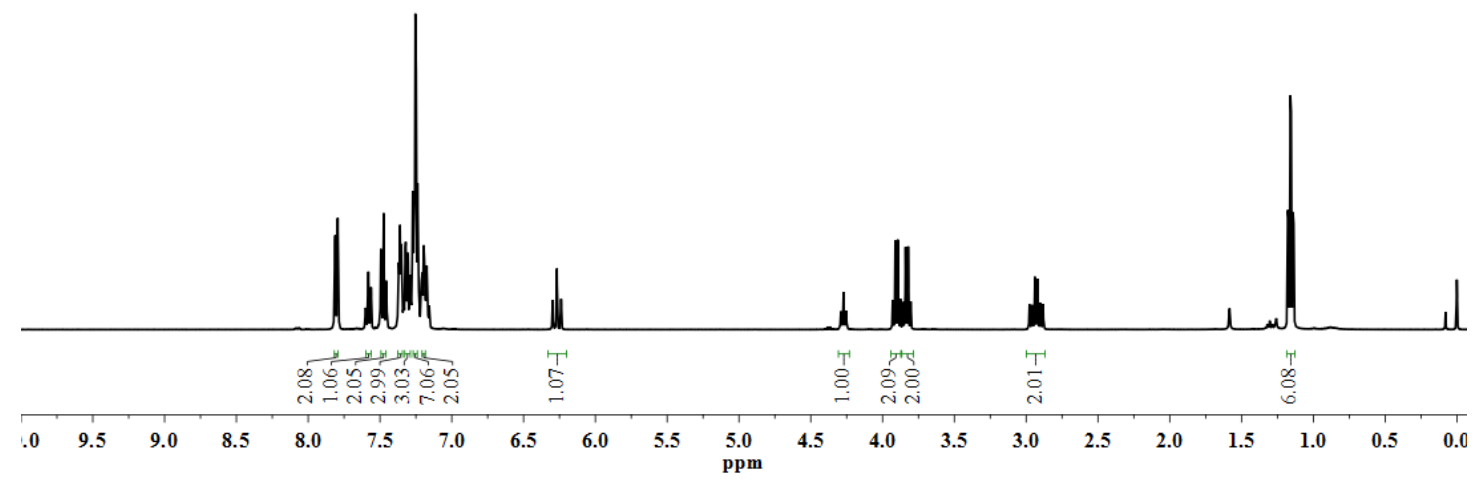

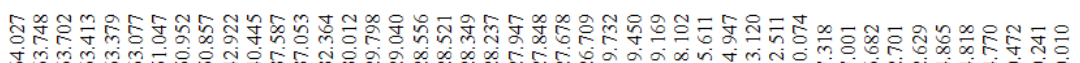

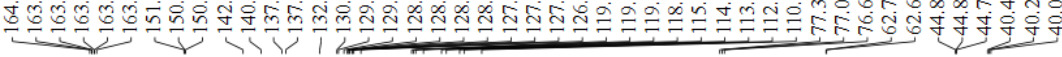

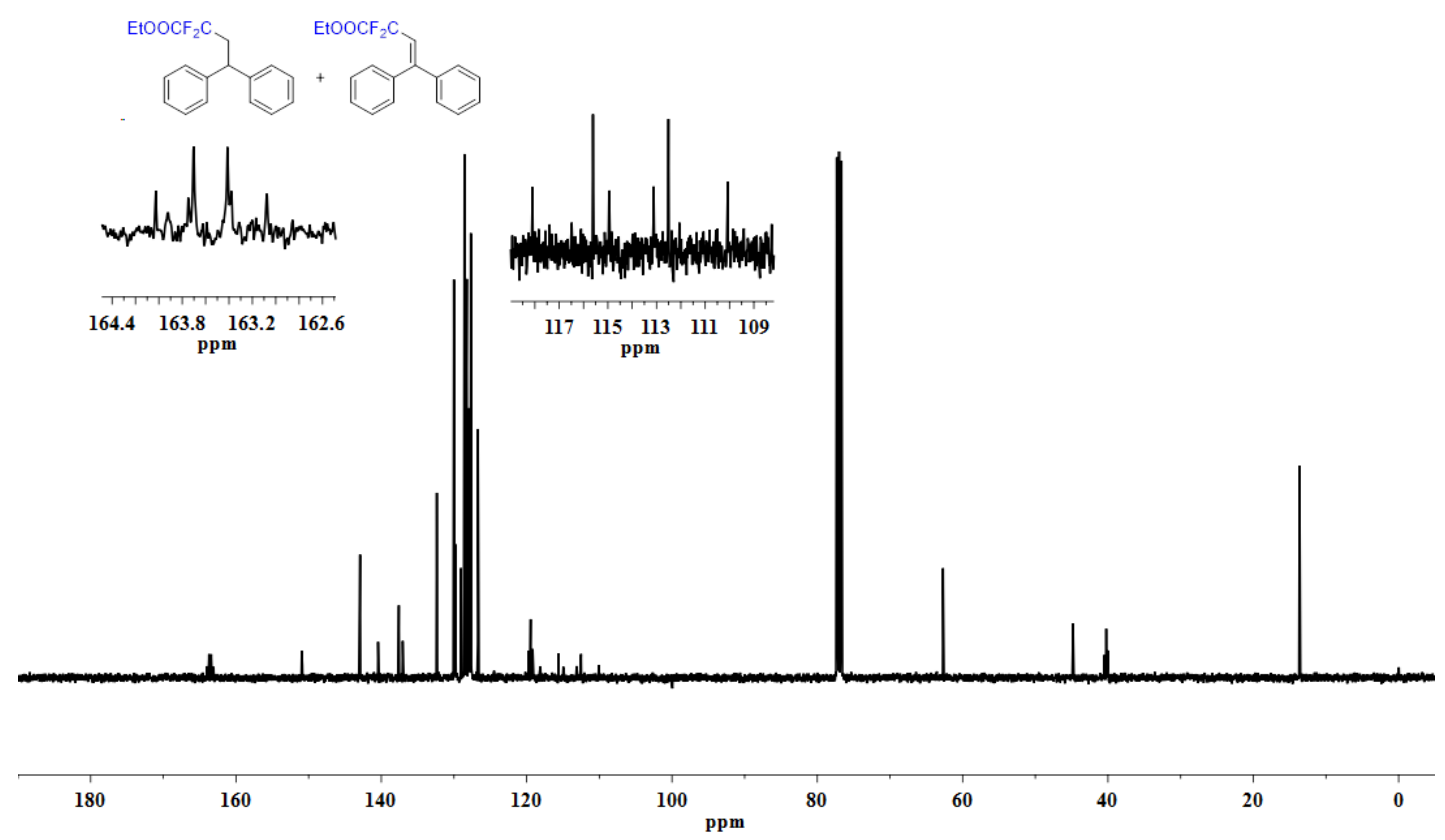


<smiles>CCOC(=O)OCCC(c1ccccc1)c1ccccc1</smiles>
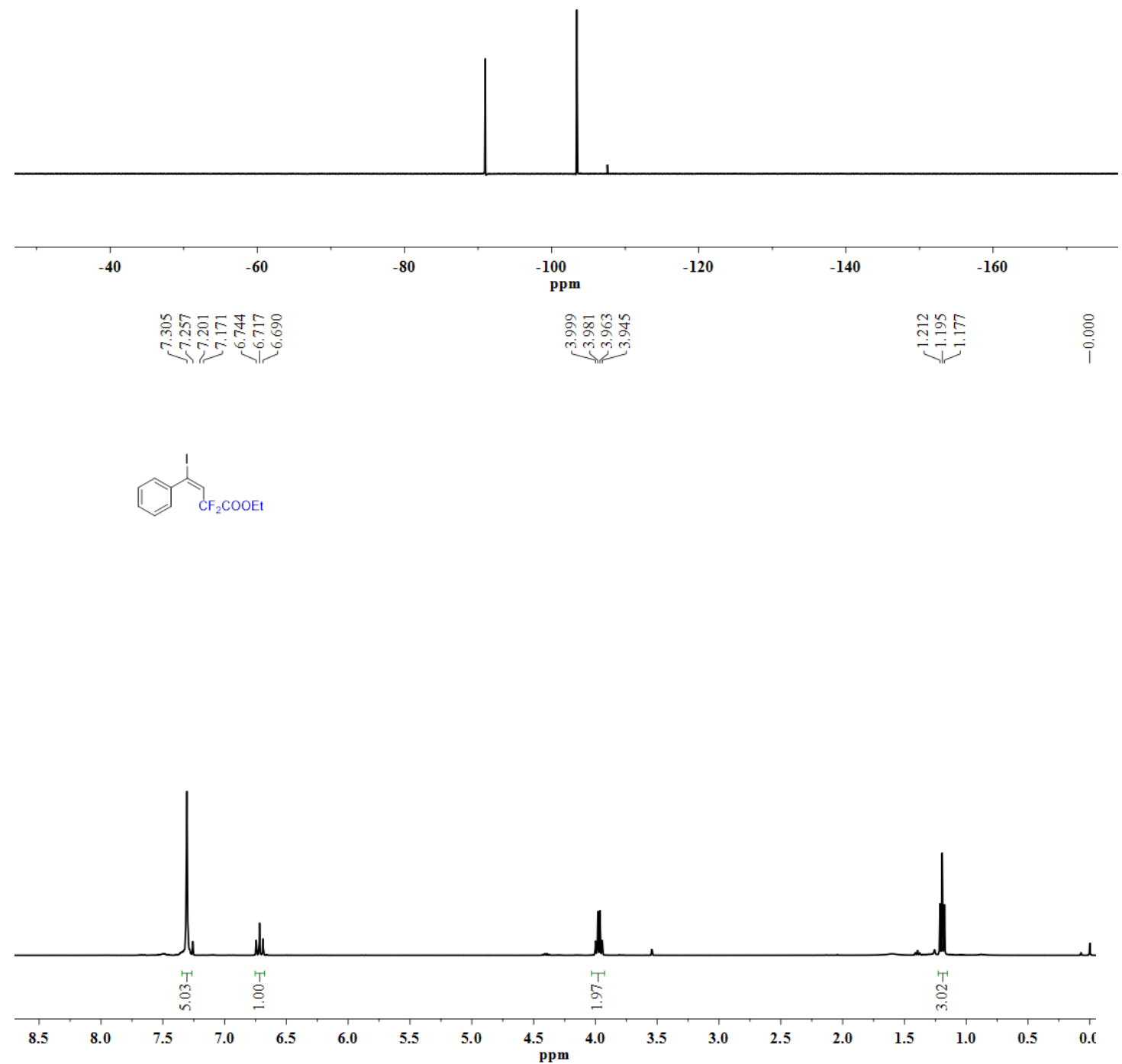

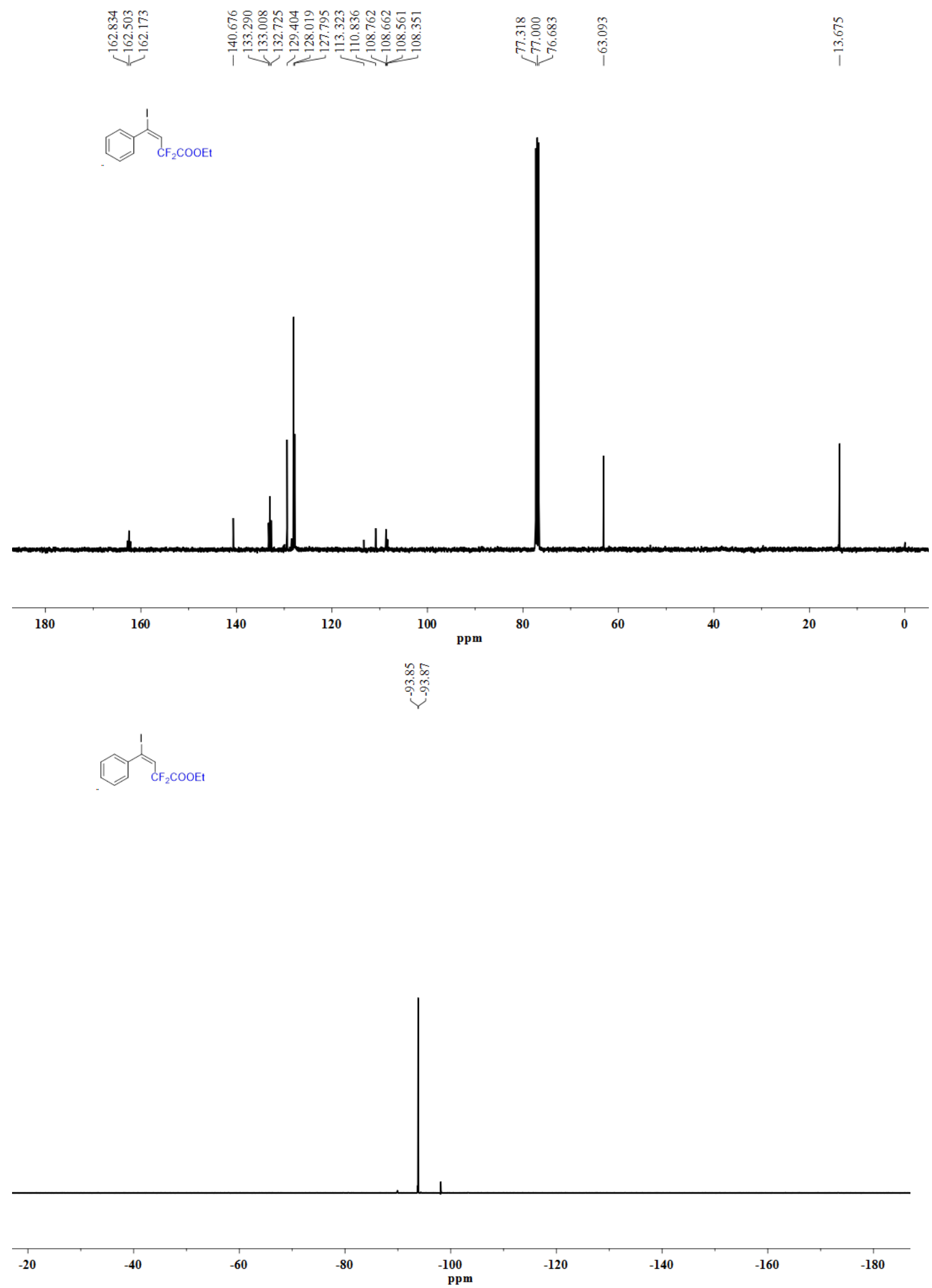